

\section{Lotoar Lerest}
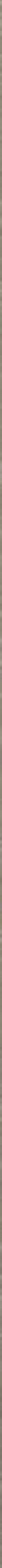

$x^{2}+2$

My. 



\section{.}





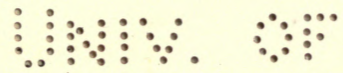

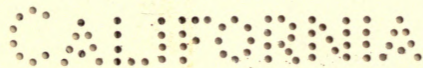




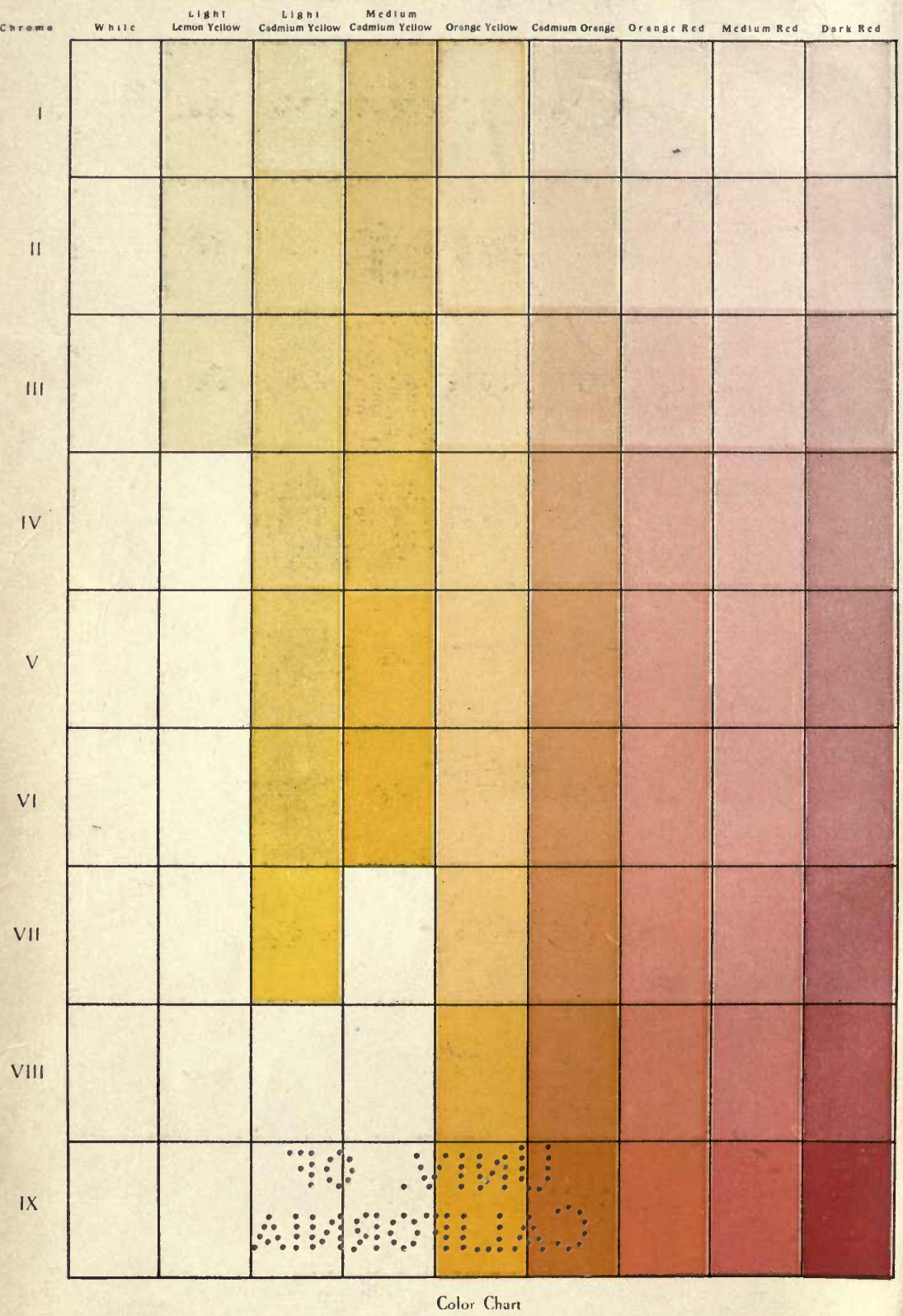

For Matching Pugments Produced by the Coccaceae 


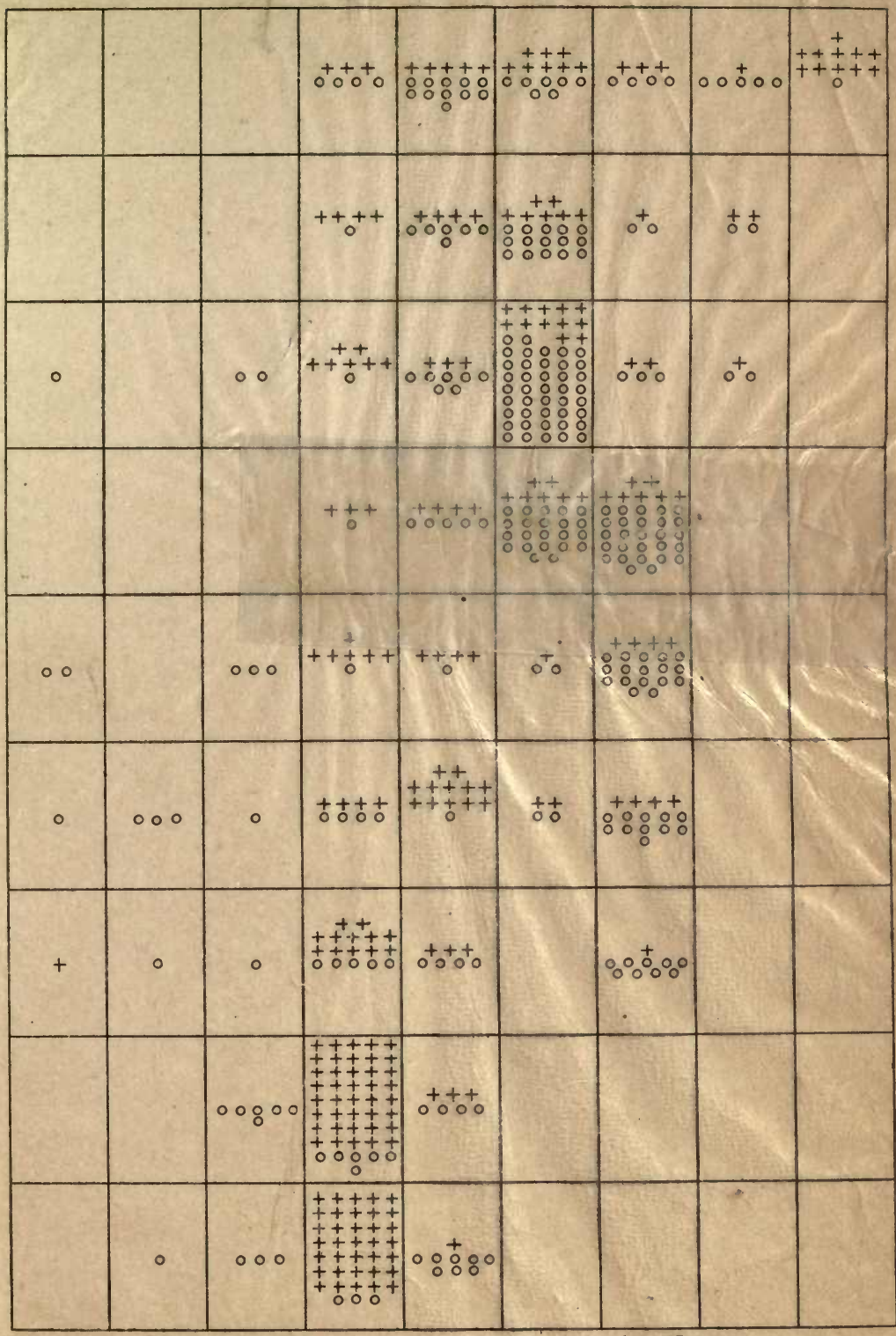

59tritqotqs 0

29tiz.5159+

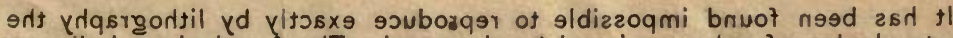

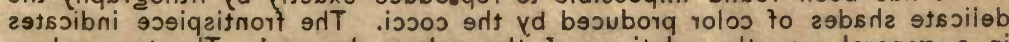

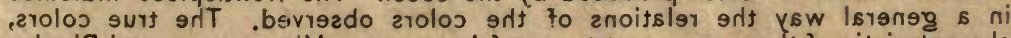

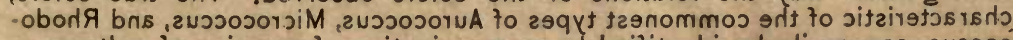

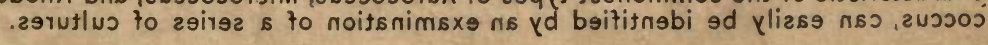




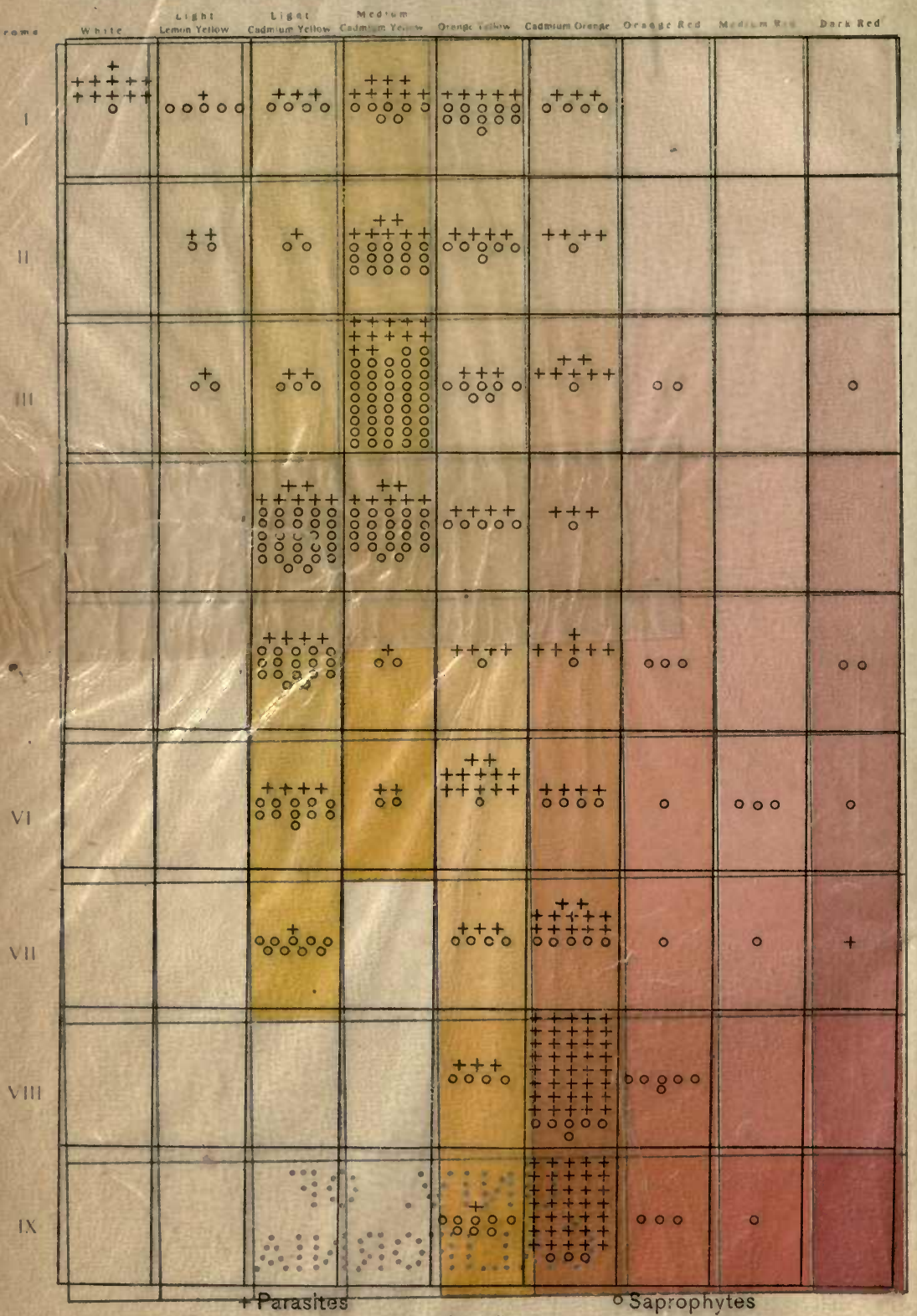

It has been found impossiple to reporgduce exact y by lithography the deiicate shades of color produced by the cocci. The frontispiece indicates in a general way the relations of the colors observed. The true culors, characteristic of the commonest types of Aurococcus, Micrococcus, and Rhodococcus, can easily be identified by an examination of a series of cultures. 




\section{The Systematic Relationships of the Coccacea}

WITH A DISCUSSION OF

THE PRINCIPLES OF BACTERIAL CLASSIFICATION

BY

CHARLES-EDWARD AMORY WINSLOW

ASSISTANT PROFESSOR OF BIOLOGY AT THE MASSACHUSETTS INSTITUTE OF TECHNOLOGY

AND

ANNE ROGERS WINSLOW

FIRSTEDITION

FIRST THOUSAND

NEW YORK

JOHN WILEY \& SONS

LONDON : CHAPMAN \& HALL, LIMITED

1908 


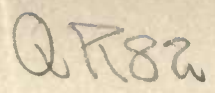

Givs

EHOLOGY

LIBRARY

\author{
Copyright, 1908, \\ BY \\ CHARLES-EDWARD AMORY WINSLOW
}

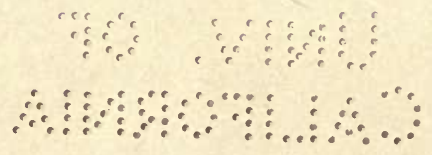

Stanbope Press

F. H. GILSON COMPANY

BOSTON. U.S.A. 


\section{WILLIAM THOMPSON SEDGWICK}

SEgKER AND INTERPRETER OF TRUTH 
"Nature exhibits to us individuals succeeding each other, but the species among them have only a relative stability, and are only temporarily invariable."-LAMARCK.

"We can only predicate and define species at all from the mere circumstance of missing links. Our species are twigs of a tree separated from the parent stem. We name and arrange them arbitrarily, in default of a means of reconstructing the whole tree, in accordance with nature's ramifications." - ElliotT Coues. 


\section{PREFACE.}

THIs book is the outgrowth of an attempt to classify certain bacteria of sanitary importance belonging to the family of the Coccacex. The task was a difficult one on account of the marked variability of the organisms in question. As in other groups of bacteria, an almost infinite number of minutely differing varieties were apparent. Biometrical methods, which have yielded such fruitful results in anthropology, and in various researches in heredity, seemed to offer the most hopeful method of attacking the problem. The attempt was therefore made to discover natural types among the Coccaceæ by a study of the numerical frequency with which various characters occur. Those characters, or combinations of characters, which are exhibited by a large number of races, as they are found under natural conditions, may be taken to mark true centers of variation, about which the rarer varieties should properly be grouped.

Five hundred different strains of Coccaceæ were isolated from various sources, - from the human body, in health and disease, from air, from water, and from 
earth, - in the laboratory of the Massachusetts Institute of Technology. All were submitted to eleven definite and, in most cases, quantitative tests; and the results were analyzed with a view to the centers about which each character varied in the series as a whole, and to the general correlation between different characters. While this work was under way it was unexpectedly extended, and its principles confirmed, by the publication of a systematic study of the streptococci by Andrewes and Horder. These observers, working in England on the finer divisions of one group of cocci, have independently arrived at the same conclusion reached by us in regard to the general systematic relationships of the bacteria: that bacterial groups can be defined, and can only be defined, by a study of the numerical frequency of various characters in a large series of cultures.

The results of the statistical study of over five hundred cultures of cocci, considered in connection with Andrewes and Horder's investigation of the streptococci, and supplemented by a careful review of previous publications on the group as a whole, furnish a reasonable conception of the relationships of the family, Coccacex, on this basis of numerical frequency types. Two main series, or subfamilies, may be distinguished: one primarily parasitic and the other saprophytic. The groups differ in morphology, staining reactions, cultural characters, and biochemical powers. Within these two sub- 
families are eight minor groups which seem to merit generic rank. Each is marked by the correlation of several apparently independent characters, and the eight form, in general, a more or less linear series, connecting such purely parasitic forms as the meningococcus with the saprophytic cocci, so common in the air. Within each genus are included three or four distinct specific types, each marked by a single peculiarity but sharing the common characters of the genus as a whole. Species and genera alike are connected by numerous intergrading varieties; but the central types in each case are defined by the preponderating frequency of their occurrence.

Extensions of this work by the examination of other cultures of cocci, by more extended tests, are much to be desired. Such investigations will no doubt add new genera and species and may show that some of those now apparently warranted are not really valid. It seems to us, however, that the results here presented offer a working basis for classifying the Coccaceæ which corresponds in the main with their natural relationships. The results so far attained have convinced us that the study of the numerical frequency of individual characters and of their mutual correlations offers a sound basis for bacterial classification; and we trust that other workers may be persuaded to adopt similar biometrical methods in studying the relationships of simple and variable forms of life. 
For assistance in the investigations upon which these conclusions are based we wish to make grateful acknowledgments to Miss Bertha I. Barker, Miss A. P. Hale, Miss M. D. Hale, and Miss Elizabeth Strongman. For the color chart reproduced as the Frontispiece of this volume we are indebted to Miss Strongman.

Brookline, MASSACHUSETts,

March, r908. 


\section{TABLE OF CONTENTS}

I. The Problem of Bacterial Classification.......... I

II. The General Systematic Relations of the Coccaceæ... $3^{\mathrm{I}}$

III. Methods Adopted in the Comparative Study of the Cocci $4 \mathrm{I}$

IV. Subfamilies and Genera of the Coccaceæ........ 76

V. The Genus Diplococcus................. I Io

VI. The Genus Ascococcus................. I 33

VII. The Genus Streptococcus............... I39

VIII. The Genus Aurococcus................ I72

IX. The Genus Albococcus................ I92

X. The Genus Micrococcus................. 209

XI. The Genus Sarcina................... 226

XII. The Genus Rhodococcus................. 239

Summary of the Genera and Species of the Coccaceæ....... 249 Key to the Genera and Species of the Coccaceæ......... 263 Bibliography........................... ${ }_{267}$ 


\section{CHAPTER I.}

\section{THE PROBLEM OF BACTERIAL CLASSIFICATION.}

THE question of the systematic relations of the bacteria has been a puzzling one since the earliest days of the science. When these minute forms were first studied by the botanists, Nägeli and many other investigators held that pleomorphism and variability were almost absolute among them. Cocci could become bacilli and bacilli, spirilla as the chance of varying environment might dictate. Gradually, however, it has become certain that there are sharp limits to variability even among these simplest of organisms. The study of pathogenic forms in connection with medical and sanitary bacteriology has greatly strengthened the conviction that, as a rule, typhoid germs descend from typhoid germs, and tubercle bacilli from tubercle bacilli. The same yellow coccus falls on gelatin plates exposed to the air, all over the world. The same spore-forming aerobes occur in every soil, the same colon bacilli crowd the intestines of animals and men in every clime. These fundamental types cannot be transformed into each other; and the controversy over pleomorphism in its cruder form, as ably presented for example in Migula's System der Bakterien (Migula, 1897) has no vitality today. 
If, however, one passes to the study of more closely related types under these main groups and attempts to determine just what forms will breed true, the problem of classification is found to be a hard one. Morphological differences among the bacteria are so slight that physiological properties must necessarily be relied upon in most cases. Yet such properties are frequently extremely variable, as in the case of pathogenic power. Among asexual forms there is no swamping effect of amphimixis to bring exceptional variations back to the specific mean. With unicellular organisms again there is no bar to the persistence of acquired characters. Bacteria respond in many ways to the direct influence of environmental conditions; and each strain transmits to a degree the impress of its recent history. Selection, too, has exceptional opportunities to modify these quickly reproducing forms. The immense number of generations which may succeed each other in a short space of time makes boundary lines as shifting as they would become among the higher plants if a dozen geological epochs were considered at once. Such variability makes detailed bacterial classification diffcult; and in some of those groups which have been most carefully studied confusion is greatest. A certain amount of "physiological pleomorphism" is strongly suspected, for example, among the allies of $B$. coli and $B$. diphtheria; and judgment has been suspended by good bacteriologists as to the existence of one, or a 
number of species among the pneumococci and the streptococci.

Under a given set of conditions many characters of many bacteria exhibit extraordinary constancy. From a broad viewpoint the fixity of type among these simple organisms is as remarkable as the deviations from it. Even such minute differences as are implied in the specific resistance of certain strains to unfavorable environmental conditions are transmitted unchanged for many generations. Yet the effect of previous variation is clearly seen when a number of different strains are compared. Such an examination discloses, not a few wellmarked species, but an indefinite series of intergrading varieties.

For these reasons the science of systematic bacteriology has remained in a notably undeveloped state. A score of large groups of bacteria have been more or less satisfactorily recognized (Flügge, I896). Certain of these groups, like those of the aerobic spore-formers, the colon bacilli, and the diphtheria bacilli, doubtless represent true natural families or genera. In one such group, that of the aerobic spore-formers, where appreciable morphological differences exist, the species and varieties have been carefully worked out by Chester (I904). For the most part, however, specific names among the bacteria mean less than nothing. The incomplete description of a vast number of identical or minutely differing forms has led to a confusion auite bewildering 
to the student of such systematic works as those of Migula (I900) and Chester (I90I). The great group of the Schizomycetes is divided by these authors into half a dozen genera, - genera based on morphological characters alone. Within these genera are hundreds of "species," grouped by a few arbitrary characters with no attempt at natural relationship. The species themselves are in most cases mere names. The descriptions attached are so incomplete that dozens of them could be eliminated as synonyms by internal evidence alone. Those which are definitely described are frequently identified by characters so slight that a thousand more species of equal value could be created by a study of other strains. It is small wonder that many bacteriologists have abandoned any attempt at a natural classification and have sought refuge in such frankly arbitrary schematic groupings as those of Fuller and Johnson (1899), Weston and Kendall (I902), and Jordan (I903). The same tendency, carried to its extreme, is shown in the decimal systems of Gage and Phelps (I903) and Kendall (I903), and in the modification of the former recently adopted by the Society of American Bacteriologists.

Such systems are valuable for certain descriptive work and for arranging and cataloguing records of cultures. Yet it is eminently desirable to supplement artificial analytical keys by a classification which shall represent the natural relationships of the bacteria 
themselves. Organic individuals have not been created in symmetrical groups differing in the presence or absence of character, $\mathrm{A}$, each group including two subgroups respectively possessing and lacking character, B. They have been developed by natural laws along irregular and complex lines, and are now related in family groupings of infinitely various kinds and degrees. A paragraph from Hall's monograph of the Compositæ of California (Hall, r9o7) applies to systematic bacteriology even more than to the classification of the higher plants: "A rational system of classification should bring out the natural relationship between the various forms; should, in other words, represent the cleavage of the larger groups into their.component parts as it has taken place in nature. Much of our recent work, however, has unfortunately consisted of a mere cutting across the grain, the result being a mass of chips - the so-called species - each being a purely artificial product and bearing no evident relationship to the others. This is commonly the result of hasty work where the perpetrator has been too busy to work out natural affinities through a comparison of inter-grading forms accompanied by field study."

Not field study, in the ordinary sense, but painstaking and sympathetic laboratory study of the organisms themselves and their "intergrading forms," is needed to make systematic bacterial relations plain; and the fruit 
of such study must be a clearer comprehension of phylogeny than can be reached by any other means.

Before attempting to discover natural groups among the bacteria it is important to distinguish the different kinds of variability which contribute to the complexity of the problem. At least three sorts of variations, so called, may easily be differentiated.

In the first place, many of the characters commonly observed are directly dependent upon immediate environmental conditions. Thus $B$. prodigiosus produces red pigment at 20 degrees and not at 37 degrees. This of course is not real variation, altho in many cases it is mistaken for it. The minute differences in the structure of colonies on gelatin, to which so much space is often devoted, are, for example, mainly due to slight variations in temperature, moisture, spissitude of the medium, etc. (Dunham, I903). A second class of variations is due to reactions between the organism and its environment in the past. These variations are the impressions left upon the organism by its recent history, such as the differences in virulence due to repeated animal inoculations, or the converse adaptations of pathogenic forms to culture media. To such differences it may be convenient to apply the term "impressed variations."

Finally there is a third class of variations, the true racial variations, which differentiate related bacterial strains under identical environmental conditions. In some groups of bacteria these racial variations are rare; 
the type seems in stable equilibrium. In other groups racial variations are almost infinite in number. Gotschlich (I903) holds that this latter condition is characteristic of organisms in the presence of a new environment; and Andrewes and Horder (1906) point out that the genera in which varieties are most numerous are the dominant genera, the groups which are at the moment succeeding in the struggle for existence. Kruse (I896) emphasizes the fact that variations are most marked under unfavorable conditions and particularly under conditions which permit of slow multiplication. Plating out old cultures, for example, shows numerous variations. On the other hand rapid transfers in a single medium tend to eliminate aberrant forms and to produce a homogeneous race, adapted to the particular medium in question. Thus our ordinary culture methods tend to eliminate variations which would naturally occur and produce an artificial uniformity and constancy.

Among the higher animals and plants racial variations appear to be of two general types, continuous or fluctuating variations, and discontinuous sports or mutations. It is probable that variations of both kinds occur among the bacteria, altho careful study of heredity among these organisms is wofully deficient. Dyar (1895) long ago made a beginning in this direction; but only a beginning. He believed that he had observed both "slight, continuous variations" and "sports or discon- 
tinuous variations," and held that the latter at least were positively inheritable.

The appearance of "sports" or mutations in bacterial cultures has been recorded her n nmber of observers; but such observations mus Itinized with care in view of the ever present anger of contamination. Neumann (1897) records the sudden appearance of sharply marked color varieties, as sectors in old and carefully sealed stab cultures of staphylococci. Beyerinck (I900), working with three distinct organisms allied to $B$. prodigiosus, observed the appearance of colorless races in each strain, the other properties of the stocks remaining unaltered. In cultures of Photobacterium indicum the same investigator noted the occurrence of two distinct mutations. In both cases the loss of normal characters was involved, and not the development of new powers. A somewhat more striking observation was communicated by Neisser (I906) to a local bacteriological society in 1906 and later published in greater detail by Massini (1907). The parent strain in this case was a bacillus of the paratyphoid group which never fermented lactose and therefore produced only colorless colonies on Endo's medium. After several days' development raised nodules appeared in the colonies. If plates were sown before these developed, all the daughter colonies were again colorless; but if plates were sown from a nodule in a colony three or four days old, two sorts of daughter colonies 
appeared, some colorless and some red. No intermediate grades were observed and cultures isolated from colorless and red colonies bred true to their respective types, altho the acid-forming mutation could always be obtained from ${ }^{t}$ old white colonies. In other cultural characters and in serum reactions fermenting and non-fermenting types were identical. In the discussion of Neisser's paper Schottelius, Kruse, Gruber, and Gotschlich reported observations of similar bacterial variations.

Slight fluctuating variations are on the other hand familiar to all bacteriologists who have examined, with care, sub-cultures from one parent strain. Conn (I900) and Sullivan (1905) studied color variations of this sort. On plates sown from a single colony they observed daughter colonies varying appreciably in shade, and by selecting extreme forms and repeating the process they were able ultimately to obtain quite distinct types. Rưžička (1899) describes the modification of a fluorescent water bacillus into a type resembling $B$. pyocyaneus by the selection of slight variations.

Whether sudden mutation, or gradual transformation of minor fluctuations, be the more important factor in the production of differing bacterial types, is still unsettled. It must be considered an open question indeed among the higher forms as well. It is sufficient in the present connection to note that variations of both sorts exist, as well as some which are hard to place definitely in 
either class. The problem for the systematist is: What weight shall be given to these variations? Shall a specific name be used for every discernible degree of variation? Or shall the members of a great group be included in one indistinguishable mass? The first course leads to a hopeless confusion of detail; the second conceals real differences in a cloud of vague generalizations. The conflict between the two views has been the notable feature of much systematic bacteriology,- as in the endless discussions as to the "Vielheit" or the "Einheit" of the streptococci.

There has recently been placed in the hands of the biologist a new instrument of research which promises at last to solve these vexatious problems. This is the statistical method, first suggested for the study of human characteristics by Quetelet ( 1846 ); specifically applied to the biological problems of variation and heredity by Galton (1889); and extended and developed in detail by Pearson and his pupils. The most important papers on this subject may be found in the files of the Philosophical Transactions of the Royal Society of London and in Biometrika. Admirable brief summaries have been prepared by Pearson (I900) and Bigelow (I904) and Vernon (1903).

From the systematic standpoint there are two important generalizations from biometry, - the statistical method of studying biology. In the first place, normal fluctuating variations, when measured in a considerable 
number of individuals, group themselves on a curve which follows the simple mathematical law of chance. All natural phenomena, governed by a large number of independent variable factors, follow this law; and form, when plotted, a Curve of Frequency, with a high point or Mode from which the curve falls off regularly, but not always symmetrically, on both sides. The height of a thousand men chosen at random forms, for example, such a curve. A few very tall men are at one end, a few very short men at the other, and from each end the numbers increase to a maximum at some intermediate point. The distances from the bull's-eye of a thousand shots fired at a target, the weight of a thousand seeds of the same plant, the altitude of a thousand hills, would form a similar curve.

If two large arrays of individuals from the same population are measured by this method the curves obtained will be nearly identical. But if arrays from diverse origins are compared significant differences appear. The position and height of the modal point, and the shape of the curve, differ. These differences are due to constant factors of heredity or environment operating on one group and not on the other. They measure the individualities of the groups as a whole, and serve to discriminate their racial types, even though particular members are indistinguishable.

Where mutually infertile species only are to be distinguished refined methods of study are scarcely neces- 
sary. When we pass beyond this point, however, statistical study is the only instrument which yields reliable results. For the examination of varieties within the species, biometry is indispensable. In the study of the human races for example this method alone has brought order out of chaos. There are tall men in Italy and dark men in Norway; but statistical work (Ripley, I899) has established the existence of three distinct European races - tall, long-headed, blond in Scandinavia; broad-headed in central Europe; short, long-headed, dark along the Mediterranean. The same biometrical methods are laying for the first time a foundation for a real science of mental and social phenomena (Thorndike, I904; Woods, I906). Wherever varietal differences are to be studied they prove of supreme value.

In a second way, too, the statistical method throws a flood of light upon the systematic relationships of variable organisms. Different racial types show different correlations of characters, as well as different centers of variation for particular characters. We may take an illustration again from anthropology. Tall men may be dark and short men may have light hair. Yet, passing from the individual to the larger group, it is shown clearly by statistical analysis that tall stature and blondness characterize one constituent racial European type, short stature and brunetness another.

The biometrical methods, which have proved so useful in the study of the races of man, promise to be of even 
greater value in the systematic analysis of types among the bacteria, where so many factors combine to preserve varietal differences on so wide a scale. If individual strains only are considered, an infinite series of differences appear. If the same strains are considered statistically, that is if the frequency of a given character be taken into account, it is apparent that certain combinations of characters are much more common than others. Measurement of almost any character by quantitative methods shows that the bacteria examined group themselves on a simple or complex curve of frequency. The modes of this curve indicate centers of variation about which the individuals fluctuate; and these centers of variation are the real systematic units of the group. The recognition of such centers, as specific types, offers the natural and satisfactory compromise between systematic multiplicity and vague generalization.

The grouping of specific types is an even more important problem than the definition of the types themselves; and here the correlation data obtained by biometrical study are of assistance. A true natural classification is tree-like and includes branches and twigs of varying grades of importance. Genera of bacteria should be aggregates of those specific types which are most nearly related; and the basis of the relationship will differ in each individual case. As Robinson has pointed out in an admirable paper on generic classification (Robinson, I906), " a difference having great 
classificatory significance in one place may be almost valueless in another." In studying any one group it is therefore necessary to examine afresh each of the various characters used for the identification of bacteria in general, to determine its local value and significance. After this has been done the selected characters should be studied by exact and definite methods in a considerable series of cultures. Finally, the results may be analyzed with two ends in view. First, each center of numerical frequency, marking a group of organisms varying about a distinct type in regard to a single definite property, may be recognized as a species. Second, those species which are bound together by the possession of a number of similar properties may be constituted as genera; and larger groups of genera, still characterized by some characters in common, may receive the rank of families or subfamilies.

It cannot of course be expected that the correlation of characters in bacteria shall be absolute. It is not absolute in any group. For example, among vertebrates, the correlation of a hairy coating with the bringing forth of living young is one of the characteristic marks of the Mammalia. Yet the Echidna and the Ornithorhynchus are classed as mammals tho they lay eggs. So, among the bacteria, correlations vary in different individuals, those properties generally correlated appearing in certain organisms in exceptional combinations. If, however, we consider, not the single 
character - not the individual organism - but the aggregate of the correlations of various properties as manifested in a.considerable series of individuals, certain well-defined systematic units appear, marked by the general association of a number of independent characteristics. Such an association can be explained only on the ground of relationship, and the types marked by the simultaneous occurrence of a number of properties may rightly be taken as the major centers from which other more aberrant individuals have varied.

It must be remembered in discussing the occurrence of correlated characters that their common presence may be due to one of two causes. The characters may be essentially independent; and may be correlated simply because ancestral forms evolved them under the action of wholly different causes. Such correlations are obviously of the highest phylogenetic importance. On the other hand, correlation may be due to the fact that the properties studied are not really independent but are so bound up together as to vary simultaneously. The selective action of the environment may produce a parallel change in both; or the two characters may be so related in the physiological balance of the organism that a change in one leads to a corresponding modification of the other.

Types marked by the correlation of truly independent characters might naturally be expected to be most significant,- they correspond more closely to the modifications 
which constitute species among the higher forms. Yet even in reference to complex plants and animals the conception of purely environmental types is gaining ground. It is not in connection with bacteria that Jordan and Kellogg (I907) speak of "Ontogenetic species held for a number of generations true to a type simply because the environment, the extrinsic façtors in the development of all the individuals in these successive generations, are the same." Ortmann (1908) has recently proposed a difference of habitat as a definite criterion of species among the higher plants. Speaking of types of orchids he says, "If further studies should show that there is segregation, geographical or ecological, between these forms, then they are species; if not, they are varieties, which fact then also will be expressed in their morphological condition, one form running into the other at least in certain parts of their ranges."

Among the bacteria ontogenetic species are common. Every bacteriologist recognizes types of structure and function definitely associated with special habitats; pathogenic and agglutinative powers for example offer striking instances of direct adaptation. Evidence accumulates too that a change of environment leads to a loss or gain of biochemical power. Peckham (1897) found that the action of colon bacilli upon pepton is highly variable. Horrocks (I903) observed that B. coli, kept in water for several months, showed only a feeble indol reaction and a delayed action on milk and neutral 
red. Twort (1907) reports that he was able by continued cultivation in sugar media to develop fermentative power in bacteria of the $B$. enteritidis type, thus breaking down the barrier between this group and that of B. coli. Schierbeck (I900) records a permanent loss of fermentative power in lactic acid bacteria exposed to the action of phenol.

In general, habitat species of definite characters are so commonly.associated with particular environments as to leave no doubt of their reality. If the same type is found again and again in the same environment, it must practically receive systematic recognition, whether its members are connected by direct phylogeny or not.

Just what rank to give to various groups among the bacteria is naturally not easy to determine. Species may often be fixed among the higher organisms by the test of mutual fertility; but here this criterion is lacking. The application of a specific name to any particular center of variation must therefore be a somewhat arbitrary act. The Linnæan nomenclature has however great practical advantages over any other system of notation. It implies no artificial order but a varying degree of family relationship; and thus corresponds most nearly to the complex truth. The rank of larger systematic units (families and genera) may well be reserved for those profound modifications which alter the whole center of gravity of the organism, while species may be defined by 
single characters which have varied by themselves without affecting other properties.

If the Linnæan system is to be used among the bacteria, however, it should be used correctly. Much of the confusion in bacteriological literature results from neglect of the simple rules of nomenclature. The principle that a species should bear two Latin names, generic and specific, and two names only, has been ignored by many medical workers; and few bacteriologists, except Migula and Chester, have respected the principle of priority, which requires that a species shall bear the name given to it in the first published description, sufficiently full for identification.

It was with the general views just outlined that we undertook in 1905 a study of the systematic relationships of the Coccaceæ by biometrical methods. Five hundred different strains of cocci were collected from various sources and each was submitted to a series of eleven definite and, in most cases, quantitative tests. The results obtained were carefully analyzed with the following objects in mind (Winslow and Rogers, I906): "We have first plotted the frequency curve for each character in order to find whether the array varies about one or several modes, and where these modes are situated, with some measure of the extent of variation about these centers. In the second place, we have calculated correlation factors for the most significant pairs of characters. Each mode on the curves of frequency may fairly be 
taken to mark a natural species or variety, and the characters which vary together must form the most important basis for the establishment of the larger groups. By such a method alone it is possible to locate those mountain peaks in the chain of bacterial variations which rightly deserve generic and specific names, altho records of the characters of individual races by the decimal system are of the greatest value in mapping out intermediate regions. Only the statistical study of numerous individuals by comparable quantitative methods can reveal the general laws of natural classification among the bacteria; and this study must be made in each group with an open mind free from arbitrary predispositions."

On the one hand, the results obtained from this investigation emphasized the extreme variability of the cocci. Almost every character measured showed a wide range of variation; and intergrading forms were so abundant as to make it wholly impossible to draw sharp and arbitrary lines of demarcation. When, on the other hand, the series was examined with the idea of discerning central types, modes on the curve of frequency, and when the correlation of the various characters was studied, the problem began to solve itself.

In the first place, two major divisions or subfamilies, within the general group of the Coccaceæ, were obviously apparent. The first group, comprising most of the forms from the body, showed, as a rule, chains and irregular cell-grouping, stained by Gram, yielded a 
meager or only fair surface growth, formed acid in carbohydrates, and produced no pigment, or a white or orange one. The other group, from earth and water for the most part, often showed packets, decolorized by Gram, grew well on artificial media, failed to ferment carbohydrates, and produced a yellow or red pigment. Each character was occasionally found in the group where it did not usually occur; but the correlation of properties in the vast majority of cases was very strong. It was a striking fact that the two chief divisions among the Coccaceæ corresponded to the two markedly different environments which exist in nature, - the body of higher organisms and the outer world. A close correspondence with environmental conditions should naturally be expected among such simple asexual organisms as the bacteria; and it increased our confidence in the reality of the groups established to find each of them localized so sharply in one or other of the two main environments.

Within the subfamilies we found a second grade of group-individuality, forming what may be called genera, marked by the association of a smaller number of characters than the subfamilies, but still defined by the correlation of several independent properties. Here, to our surprise, the property of chromogenesis appeared of supreme importance.

Six groups of species, which seemed to us of generic rank; appeared among the cocci studied. Each was 
marked by the correlation of several distinct characters. The first type, of which the ordinary parasitic streptococci are examples, produces a faint veil-like growth on agar, shows under the microscope pairs or chains of small groups of cells, generally stains by Gram, fails to . reduce nitrates or liquefy gelatin, and produces a strongly 1 acid reaction in dextrose and lactose broth.* The second and third. types include the forms characterized by orange and white growth, respectively (the common staphylococci of the skin). For these two types we have suggested the generic names, Aurococcus and Albococcus. Both resemble the streptococci in parasitic habit and generally positive Gram reaction. Both, like the streptococci, differ from the other three types in the fact that they occur in small cell aggregates (never in packets), and in their power of fermenting dextrose and lactose, altho they show larger cell aggregates and form less acid than the streptococci. Nitrate reducers and gelatin liquefiers are found in both groups, but the : orange liquefiers act much more vigorously than do: those of any other type. The frequency curves for this character are plotted in Fig. III.

The other three groups of the cocci include the saprophytic, yellow and red chromogenic forms. Cocci of both types may of course be found on the body, but they are the prevalent forms in water and earth, as the white

* The quantitative basis of these definitions is discussed in Chapter III. 
and orange chromogens are the common cocci of the skin. Both yellow and red types frequently show the packet grouping; and in the yellow type we have continued the separation into two genera, Micrococcus and Sarcina, which is now in almost universal use. In all other respects the packet-forming and non-packetforming -yellow series are closely parallel. Both types form a good growth on media, of a lemon yellow color, and the distribution of these organisms on the color chart, reproduced as the frontispiece of this volume, shows that the type center of the yellow saprophytes is separate and distinct from that of the orange parasitic forms. Both micrococci and sarcinæ are generally Gram negative. They form typically a slight acidity in dextrose broth and none in lactose broth; and the frequency curves for dextrose in Fig. II show a remarkable parallelism between the two genera. Finally the red chromogens, which we have grouped under the new genus, Rhodococcus, form another distinct type by themselves. They resemble the yellow cocci in saprophytic habit, negative Gram reaction, and low fermentative

I power. They differ from them in their peculiar pigment, in the absence of vigorous liquefying action, and in the fact that they reduce nitrates, if at all, to nitrites

$\checkmark$ and not to ammonia.

The result of our study was to convince us that these six types represent true natural groups of the Coccaceæ, each marked by the occurrence of a peculiar complex 
of characters, - the whole forming a more or less linear series from such strict parasites as the diplococci and certain streptococci to the vigorous saprophytic rhodococci. Within each genus several distinct species can be defined by separate type centers of variation in single properties. If our conclusions are justified, the arrangement approaches a natural phylogenetic one.

It is interesting to notice that particular tendencies seem to lie latent in cocci of all the main groups. Thus the property of liquefying gelatin appears to some extent in each of the six genera mentioned, altho it is rare in Streptococcus and very feeble in Rhodococcus. The power of reducing nitrates is lacking in most strains of each genus (except Rhodococcus); but in each genus except Streptococcus some cultures show reduction. Zooglea and capsule formation too, altho appearing most markedly in the genus Diplococcus and among the Ascococcus forms of the sugar refineries, crop out in one species of Albococcus and in rare strains of Sarcina. Many of these properties are apparently latent in bacteria of widely separated groups outside the Coccaceæ. Thus Molisch (1907) finds zooglea formation and the sarcina grouping, as well as the fundamental bacterial forms of cocci, bacilli, and spirilla, among the purple bacteria, whose peculiar pigment and characteristic physiology and distribution mark them off as a separate order.

It was the success of biometrical methods as applied 
to problems in theoretical biology and anthropology which led us to attempt the solution of the vexed question of bacterial relationships by these means. In the same year in which our paper was published, Andrewes and Horder (I906) in England presented a study of species in the genus Streptococcus, based on identical principles but apparently arrived at in independence of the work of others in any field. The following statement of the case by these authors shows an interesting parallelism with the contemporaneous passage quoted from our own paper on page I8; but Andrewes and Horder have expressed the principle of biometrical classification with peculiar felicity. After pointing out the difficulties in the way of classifying the streptococci they proceed as follows:

"There was, however, one guide which, as in all such taxonomic problems, proved of the greatest help namely, the numerical frequency of occurrence of any given type. When any arbitrary set of characters is taken as a basis for the classification of a group of natural objects the same phenomena are usually seen large groups of like objects connected by small groups which differ from them in only one or two particulars. If the numerical frequency of each individual like the group is represented by the proportional height of a vertical line and the lines are arranged in series the commoner types stand out boldly above the rarer ones. Only in nature they are plotted out, not in linear series. 
but in space of two dimensions, as it were, so that the common types stand out as mountain tops above their fellows, each mountain connected by valleys of intermediate types with many of its neighbors. If now the mountains were cut off by a horizontal plane half way up their sides and attention were paid only to the mountain tops, disregarding the valleys, we should have the popular conception of species. The biologist, on the contrary, is more concerned with the intermediate types in the valleys, as illustrating variation and the connection between allied species. In some groups of plants and animals the mountains are few and high and the valleys very deep. These are the groups which are, so to speak, in a stationary condition - which are not rapidly varying and adapting themselves to new conditions. In other groups, which biologists call 'dominant genera,' the mountain tops are numerous but not so high and separated by only shallow valleys; these are the groups which are at the moment succeeding in the struggle for existence." (Andrewes and Horder, I906.)

By the application of these methods, Andrewes and - Horder succeeded in bringing a reasonable order out of the chaos which had shrouded the systematic relationships of the streptococci. Years of previous work by scores of able bacteriologists had only made it clear that this genus included an innumerable series of varieties; not a single step had been taken towards the arrangement of these varieties in natural groups. The 
statistical method was essential for the solution of the problem.

Andrewes and Horder analyzed the records of I 200 different strains of streptococci which were available through the painstaking investigations of Gordon and Houston. The data at their disposal included tests of fermentative power in a considerable number of carbohydrate media. The observations were unfortunately not quantitative; but a large number of substances were tested. In spite of the bewildering variations exhibited by individual cultures the numerical frequency of certain types was at once apparent. Seven such types, marked by definite combinations of characters, formed peaks in the mountain chain of varieties, the rarer variations grouping themselves about the main centers and differing from them by comparatively slight degrees. Furthermore, each type center was associated with a definite habitat, one with the intestine of the Herbivora, one with the human throat, one with pathological conditions, etc. The results of this study will be reviewed more fully in Chapter VII. It is pioneer work and will doubtless require revision in many respects; but its authors have succeeded in mapping out for the first time a classification of the streptococci which bears evidence of correspondence with the natural phylogenetic relations of the group. It complements our own work which dealt mainly with the staphylococci and the saprophytic forms. Together, the two inves- 
tigations cover the family as a whole with reasonable fullness.

The success of Andrewes and Horder in classifying the streptococci is strong evidence of the value of biometrical methods in systematic bacteriology. Their results and our own have led us to the conviction that these methods offer the key to the natural relationships of the bacteria. A few general rules of procedure must however be accepted before detailed application of statistical methods can be fruitful.

In the first place it must be admitted, before any progress is possible, that physiological characters are as important as morphological characters in this group; and that they alone, if of sufficient degree, may serve for specific and generic definition. One of the greatest drawbacks to systematic bacteriology has been the attempt to find morphological differences where none exist, and the corresponding failure to study physiological characters with adequate exactness. Yet it is precisely along the lines of metabolism that the bacteria have attained their extraordinary degree of differentiation. Other plants and animals, under the stress of natural selection, have developed complex systems of organs for procuring food of certain limited kinds and for escaping harmful environmental conditions. The bacteria have maintained themselves by acquiring the power of assimilating simple and abundant foods of varied sorts, with a special resistance against cold and 
heat and dryness. There is as wide a difference in metabolism between the pneumococcus and a nitrifying bacterium as there is in morphology between the kangaroo and man. Physiological differences in one case are quite as significant as morphological differences in the other.

Migula (I897) strongly maintained this ground with regard to bacterial species and further pointed out that the distinction between physiology and morphology is merely an artificial one, since any physiological difference must have a morphological basis, however far below the limit of our vision the difference may lie. Curiously enough, he, and practically all other systematic bacteriologists, have submitted to the arbitrary morphological criterion for genera tho repudiating it for species. It seems clear, however, that the same reasoning should hold in both cases. If certain differences mark species, more pronounced differences of the same general nature, which characterize certain groups of species, may properly be recognized as of generic importance.

Secondly, an absolute requisite in regard to bacterial characters, morphological or physiological, is that they should be definite, and capable of so clear a description that they can be identified without difficulty by other observers. Vague descriptions of large size, slow liquefaction of gelatin, yellow color, etc., are of little service in a group where so many minute variations exist, unless accompanied by definite statements as to exactly 
what is implied by these terms. Quantitative measurements should be made wherever possible: if this grade of exactness cannot be attained color charts or full explanation of the meaning of each term should be forthcoming.

Finally, if our view is correct, bacterial types should never be described on-the strength of an examination of single individual strains; but only after a comparative study of the numerical frequency of each particular character in a considerable series of cultures. When the measurement of such a property as gelatin liquefaction in a given series of organisms shows distinct bimodal curves (as in Fig. III on page 100), each mode may be considered a distinct type. In the curves of Fig. III, for example, it is clear that two centers of variation exist in each of the three groups studied one a liquefying and the other a non-liquefying type. Often a quantitative comparative study is unnecessary, in order that a type difference of this sort should be made clear. For example, a survey of medical literature alone is sufficient to show that the diplococcus of meningitis and the diplococcus of pneumonia constitute two real type centers in the general group to which they belong. A vast majority of the organisms associated with one pathological condition are flattened, biscuit-shaped pairs of cells which decolorize by Gram and exhibit mutual serum reactions. A vast majority of the cocci from the other condition are elongated lanceolate cells which 
stain by Gram, and show a second set of group reactions to immune sera. The principle which differentiates these specific types is the same as that involved in the distinction of the liquefying and non-liquefying cocci; but the difference in this case is so marked as not to require statistical demonstration.

The essential viewpoint which our biometrical study of the cocci has developed may be briefly restated as follows.

Bacteria show, in many groups at least, great variations. A comparative study of a considerable number of strains shows that certain characters, or combinations of characters, occur with special numerical frequency. These frequency types represent the centers about which related organisms are varying. Each type center which is distinguished from other type centers by the exhibition of a definitely measurable character may be given the rank of a species. Genera may be constituted for nearly related groups of species which exhibit characters in common. Such species and genera represent real, natural groups of bacteria, which owe their similarity either to community of descent (phylogenetic species) or to similar pressure of environment (ontogenetic species). 


\section{CHAPTER II.}

\section{THE GENERAL SYSTEMATIC RELATIONS OF THE COCCACE E.}

THE bacteria which generally appear in a spherical form were called Coccaceen by Zopf (I885); he was an ardent believer in the transformation of bacterial species, and considered the coccus-shape as a transitory form which any bacteria might assume under favorable conditions. As the general stability of bacterial characters was gradually established by more careful observations, the name Coccaceæ was applied to the spherical bacteria, which were found to constitute a well-marked natural group. The family is defined by Migula (I900) as follows:

Family Coccace e (Zopf) Migula. Cells, in their free condition, spherical; during division somewhat elliptical. In the latter condition, division has already set in, altho it may not be apparent. Division in one, two, or three planes, without previous elongation of the cells. If the cells remain in contact after division they are frequently flattened in the plane of division. Motility is present only in a few forms. Formation of endospores appears to be absent or very rare.

Recent investigations of these bacteria make it in- 
creasingly clear that the cocci form a natural group of unusual definiteness. It is difficult to add to Migula's description any other single character which is common to the family. Yet a consideration of the properties of the members of the group makes it clear that they are mutually interrelated and all sharply separated from the rod-shaped bacteria, except perhaps at one end of the series which they form.

The subdivisions of the Coccaceæ, however, have been less satisfactorily established than the status of the family itself. No other group of the bacteria has been burdened with more generic names. Ascococcus, Bacteridium, Cohnia, Diplococcus, Gonococcus, Hyalococcus, Lampropedia, Leucocystis, Leuconostoc, Merismopedia, Merista, Micrococcus, Microhaloa, Monas, Pediococcus, Planococcus, Planosarcina, Sarcina, Staphylococcus, Streptococcus, Tetracoccus, is a partial list. Many of these names date back to the early days of bacteriology and are due to the attempts of botanists, trained in other fields than bacteriology, to create genera on slight morphological grounds alone. Merismopedia, Hyalococcus, Staphylococcus, and Tetracoccus are examples of this sort; and Fischer's Pediococcus is a more modern instance of the same tendency. Migula (1897) made an exhaustive review of the earlier attempts at generic classification of the cocci and eliminated as synonyms, or imperfectly characterized, all previous genera but three, - Streptococcus, Micrococcus, and Sarcina. He added two new genera, 
Planococcus and Planosarcina, for the motile forms, and these five genera form the basis of his detailed systematic treatment of the group (Migula, I900). Chester (I901) accepted Migula's classification in its general outline.

Planococcus and Planosarcina, the genera characterized by motility, are based on three or four exceptional strains of cocci which differ from related forms in the possession of this single property. Streptococcus, Micrococcus, and Sarcina on the other hand are large and unwieldy genera. They are commonly defined by morphological characters alone, all chain-forming cocci being placed in the genus Streptococcus, all packet-forming cocci in the genus Sarcina, and the rest in the great group Micrococcus. In the latter genus widely dissimilar forms, of diverse habit and various characteristics, have naturally accumulated.

The inclusion of so many species under three large genera renders some grouping of the specific forms within the genus an absolute necessity. No attempt has been made by most observers, however, to distinguish natural groups. The general plan adopted has been an arbitrary division of the genera by certain salient single characters, - a scheme resembling the analytical keys used as a supplement to true classification among the higher plants. Thus Flügge (I886) described 44 species of micrococci and grouped them, first, according to gelatin liquefaction and, second, according to the 
color of the colonies. The unnatural collocation of organisms produced at the end of such an arbitrary scheme may be imagined. Under the white nonliquefying group, for example, the streptococci were distinguished by their small isolated colonies from another group which included diplococci, micrococci, and sarcinæ. Dyar (I895), too, made his primary division of the micrococci into liquefying and nonliquefying forms. Each of these groups was then separated according to acid production, and still finer divisions were based on chromogenesis and other characters.

Migula (I900), on the other hand, makes his primary division of the cocci, which have been cultivated on gelatin, according to chromogenesis. Under Micrococcus there are seven primary divisions; white non-liquefiers, 60 species; white liquefiers, 4I species; yellow nonliquefiers, 20 species; yellow liquefiers, 35 species; red non-liquefiers, II species; red liquefiers, I2 species; blue and violet forms, 3 species. Similarly his genus Sarcina includes 6 white liquefiers, 9 white non-liquefiers, I6 yellow liquefiers, I4 yellow non-liquefiers, 2 brown forms, and 5 red forms.

Chester's classification (Chester, IgoI), is based on the same general principles as that of Migula; but within the various color classes are sub-groups marked by the vigor of growth at various temperatures, and by the appearance of surface growths on various media. This 
marks a distinct step forward. He separates such forms as the gonococcus and $M$. catarrhalis from the other micrococci by their feeble growth on ordinary culture media; and distinguishes the "doubtfully chromogenic forms" which produce a light yellow growth from the "distinctly chromogenic" or yellowish orange forms.

In I905, the authors made a careful comparison of the published description of 445 supposedly distinct species of cocci as given by Cohn, Migula, Flügge, Chester, Sternberg, Lehmann and Neumann, Engler and Prantl, Rabenhorst, Frankland, LeGros, and Woodhead. Examination of the literature alone, in the light of a general knowledge of the characteristics of the Coccacex, was sufficient to show that most of the 445 specific names had no adequate basis for existence. Slight variations in morphology (the size of cells, the length of chains, etc.), slight peculiarities in the appearance of colonies on gelatin, slight differences in vigor of growth at certain temperatures and on certain media, taken by themselves, are clearly inadequate for the establishment of bacterial species. If such observations are made definite by quantitative measurements and tied together by studies of correlation, they may prove most helpful; but exact data analyzed in this way have hitherto been wholly lacking. Almost the only clean-cut facts noted in the published descriptions of species among the cocci, collected by Migula and Chester in their standard works, are the relation of the organisms to gelatin, their action upon sugars, 
and their pigment production and general vigor of growth. By using these characters, with one or two others which were of importance in special cases, we made a tentative grouping of the 445 described forms under 3 I types (Winslow and Rogers, I905).

Some of the $3^{I}$ types, recognized from the literature, in this preliminary communication, have since proved to be artificial in view of a comparative study of the cocci themselves. The main points brought out at the early stages of the investigation still, however, seem to us well founded. Among these, was the conclusion that the family as a whole may be divided into two main groups, one of parasitic, the other of saprophytic habit. Pathogenic power is one of the properties which vary most easily among the bacteria; and from the systematic standpoint it has appeared to Migula (1897) and others to be of slight importance. Nevertheless, it is quite clear that certain well-defined natural groups of bacteria are generally associated with the peculiar environment offered by the epithelial surfaces of the animal body. It is necessary to make a distinction between parasitism and pathogenicity. The parasitic habit, the adaptation to life on the body surfaces of the higher animals, marks off certain important groups of the bacteria. Within these groups the actively pathogenic forms and those which live harmlessly on the epithelia are closely related. Among the Coccaceæ particularly, the pathogenic powers appear to come and go with special ease; but the para- 
sitic habit is, on the whole, correlated with quite distinct morphological and physiological peculiarities. In our first paper on this subject we pointed out that the family of the Coccaceæ is divisible on this basis "into two subfamilies. The first, for which we suggest the name Paracoccaceæ (paratrophic cocci), includes Diplococcus and Streptococcus, parasitic forms which do not develop abundant growth on artificial media and which thrive better under anaerobic than under aerobic conditions, and appear in small cell aggregates of pairs or chains. The second subfamily, the Metacoccaceæ (metatrophic cocci), includes Micrococcus, Sarcina, and Ascococcus; saprophytic or semi-saprophytic types which are aerobic and form abundant surface growths of large cell groups."

The study of the literature alone led us to suggest the restoration of the genera, Diplococcus, Weichselbaum, and Ascococcus, Cohn. The reasons for this course will be discussed somewhat fully in succeeding chapters. The former genus was evidently characterized by strictly parasitic habit, very feeble growth on artificial media, and the possession of capsules under proper conditions, as well as by its diplococcoid form. The organisms generally classed in the genus Streptococcus, which obviously form a second natural group of considerable definiteness, are related more closely to the genus Diplococcus than to the rest of the family. Like the diplococci they are normally parasitic on the animal body. Their growth on artificial media, tho more vigorous than that of the 
diplococci, is much more feeble than that of other forms, and their fermentative powers are high. Andrewes (I906) has recently emphasized the essentially parasitic nature of the genus Streptococcus, and has ably outlined its general relations to other groups of the Coccaceæ. His view that the streptococci have descended from the saprophytic Metacoccaceæ is suggestive; and his theory that certain parasitic bacilli have in turn been derived from the streptococci is not improbable.

It must be emphasized that both diplococci and streptococci are recognized by the correlated occurrence of various characteristics and not by the mere presence of pairs or chains of cells. The morphological criterion alone must not be interpreted literally. Cocci of all genera occur at times in pairs; and typical streptococci fail to show well-marked chains in many media.

With regard to Ascococcus we concluded in our earlier investigation: "The mere property of zooglea formation should not be considered of generic importance, but the few peculiar species which are capable of growing under purely saprophytic conditions and producing large gelatinous masses, are so far marked off from other cocci as to warrant, in our judgment, the retention of Cohn's genus." We have not examined these organisms at first hand. Further study of the work of others has convinced us, however, that Ascococcus should probably be referred to the Paracoccaceæ rather than to the Metacoccaceæ. The reasons for this conclusion will be discussed in 
Chapter VI. We still feel that the combination of chain formation, with the production of large zooglea masses, the growth under saprophytic conditions, and active fermentative powers, constitutes a well-marked natural group.

The genera Planococcus and Planosarcina, founded upon the single characteristic of the possession of flagella, seem on the other hand of somewhat doubtful value. The slow revolution and steady translation observed by Ali-Cohen and Migula as associated with flagella, is certainly a phenomenon distinct from the irregular vibratory and rotary movements noted by other observers. True motility appears in a few, and only in a few, cocci; but the resemblance between motile and non-motile forms is .so close in all other characters that this property scarcely seems of generic importance.

These considerations led us at first to recognize five genera among the Coccaceæ: Diplococcus, Streptococcus, Micrococcus, Sarcina, and Ascococcus; and these genera appeared to be arranged in a natural serial order. Diplococcus is strictly parasitic and commonly produces only aggregates of two cells. Streptococcus, also normally parasitic, thrives better, tho still not luxuriantly, on artificial media, and its typical growth form is a chain. Both these genera form acid in sugar media. Micrococcus includes both pathogenic and non-pathogenic forms, but all grow abundantly on gelatin and agar, in rather large, irregular cell aggregates, while some produce acid, and some, alkali, in milk. Sarcina shows 
further development in the same direction, its growth form being larger and produced by three planes of division, its saprophytic habit being more marked (no truly pathogenic forms being known to exist), with the power of acid production generally wanting. These genera seemed to mark important phylogenetic stages in a series of types of Coccaceæ ranging from such strict parasites as D. Weichselbaumii, through intermediate streptococci and micrococci to the vigorously growing saprophytic sarcinæ. 


\section{CHAPTER III.}

\section{METHODS ADOPTED IN THE COMPARATIVE STUDY OF THE COCCI.}

THE general relationships, discussed in the last chapter, were suggested by the descriptions of Coccaceæ available in the literature. In order to establish our conclusions, and to extend the analysis to smaller groups, it was necessary to carry out a comparative study of a considerable series of cultures of cocci from various sources. Such a study, covering 500 different strains of cocci, was made in 1905-06. Our aim was to observe as many definitely measurable characters as possible; to find the centers of variation marked by the modes of the frequency curves obtained; and to discover the correlations existing between the various characters. According to the views set forth in Chapter I, we believed that each distinct center of variation could be considered as marking a specific type, while the species which were identical in several correlated characters could be considered as forming a single genus. The general results of this investigation have been already published (Winslow and Rogers, rgo6), in brief form.

We included in our study representatives of only three 
of the five genera to which reference has been made above. The organisms belonging to the genus Diplococcus do not easily lend themselves to comparative study on account of the difficulty with which they may be cultivated; and representatives of the genus Ascococcus occur only in certain peculiar habitats. We have limited our study to forms which can be found in ordinary environments and which may be cultivated on ordinary laboratory media; that is, to the genera Streptococcus, Micrococcus, and Sarcina, as ordinarily understood.

We procured our cultures, in approximately equal proportions, from five different sources: from the internal tissues of the diseased human body, from the outer surfaces of the normal human body, from water, from earth, and from air. Cultures classed under Habitat I, the tissues of the diseased body, were obtained chiefly from the Boston City Hospital, and the Massachusetts General Hospital, of Boston and the Johns Hopkins Hospital of Baltimore. The cultures classed under Habitat II, surfaces of the normal body, were obtained from three sources. A considerable number were isolated from serum tubes which had been received by the Boston Board of Health for diphtheria diagnosis but which had shown negative results. Another series of cocci was isolated from the hands of students in the Massachusetts Institute of Technology. Finally a small number of cultures were obtained from excreta of man and animals. Under Habitat III, cultures were obtained from a wide 
variety of natural waters - public supplies, streams, ponds, pools, shallow wells, driven wells, and the sea. Samples were taken, as far as possible, only from sources considered free from pollution. Under Habitat IV, organisms were isolated from various samples of earth, loam, clay, sand, etc., obtained mainly in different regions of eastern Massachusetts. The cultures grouped under Habitat $\mathrm{V}$ were taken from plates exposed to the air of the laboratory or of the city streets; and here are also included certain organisms of unknown origin which appeared as contaminations, or for whose previous history we had no record.

In each case the sample to be studied was first plated on agar and incubated at 20 degrees. Colonies which looked like cocci (not possessing, for example, the characters of such well-marked forms as $B$. mesentericus, B. Zopfii, or B. fluorescens), were fished to agar streaks; from each individual source only one culture was taken, unless several distinct types of colonies appeared. The agar streak cultures were examined under the microscope and, if apparently cocci, were replated in order to insure their purity, again transferred to agar streaks, and again examined under the microscope. All this preliminary work was carried out at 20 degrees, and the stock cultures finally obtained were kept on agar at the same temperature. There can be no doubt that by this method of procedure we failed to obtain many of the more strictly parasitic streptococci which grow only feebly on 


\section{RELATIONSHIPS OF THE COCCACEA}

solid media and are most active at a temperature of 37 degrees. This fact must be taken into account in interpreting our results. For Micrococcus and Sarcina, however, the series should be fairly representative.

The characters ordinarily used in descriptive bacteriology are few enough, particularly in a group of such simple morphology and limited biochemical powers as the Coccaceæ. This number must be still further reduced, however, when we come to inquire which of them really indicate constant and independent variations. In the first place, it is necessary to eliminate properties which are due mainly to the character of the medium and the conditions of incubation. Minute differences in the appearance of colonies on gelatin, which form the basis for a large number of German descriptions, fall mainly under this head. Secondly, many characters, while really belonging to the organism itself at a given moment, are so easily modified by cultivation under other conditions as to be of minor value in systematic work. Among the cocci, pathogenicity is a property of this sort. In the third place, it is obviously unnecessary to give independent weight to characters which are simply the indirect result of other properties already recorded. Thus among the cocci differences in broth cultures are closely connected with the size of the cell aggregates. Organisms growing in large groups, like most of the sarcinæ, produce heavy sediment and often colony-like groups on the walls of the tube, while those in 
which the cells readily separate exhibit a more diffuse turbidity. Plate cultures add little more information than may be obtained by a careful scrutiny of stabs and streaks; and the growths on potato and blood serum in many groups of bacteria, and particularly among the cocci, are only valuable as 'measures of that extremely variable quality, the general vigor of the culture.

The considerations which have influenced us in the selection of characters for study among the Coccaceæ may be conveniently arranged in the order and under the headings of the Report of the Committee on Standard Methods of Water Analysis to the Laboratory Section of the American Public Health Association (I905).

\section{Morphological Characters.}

The form of the individual cell furnishes no help in the classification of the Coccaceæ, since under favorable conditions all appear as regular spheres. Irregular oval cells occur at times, particularly in cultures freshly isolated from the throat or alimentary tract, but the form usually becomes normal after cultivation.

Manner of grouping. 'The grouping of the cell elements offers a character of considerable importance among these bacteria. Individual cultures exhibit a distinct tendency to occur in one of four forms - either in pairs, chains, masses, or packets. The grouping is somewhat influenced by the age of the culture, and by the kind of medium on which it is grown; and even the 
same culture will show wide variations. For instance, streptococci occur singly, in pairs, chains, and small masses; but the most frequent arrangement, and that observed under the most favorable conditions (liquid - media), is in chains. Again, sarcinæ occur singly, in pairs, and in small masses, as well as in packets, yet the typical form is the sarcina-packet. Cocci grown on Nährstoff regularly occur in plates, and usually capsulated ones.

In a number of preliminary studies we compared the groupings of the same cultures in various media and under various conditions, examining cultures of different ages,- from nutrient broth, sugar broth, pepton solution, hay infusions, nutrient agar, and gelatin, and acid and alkalin gelatin. Cultures more than two weeks old showed abnormalities, both in the individual cell and in its grouping. With this exception, the differences observed were slight. The only apparent effect of the medium upon grouping was a more distinct development of chains in liquid cultures. Organisms which appear as long chains in fresh broth may. show only short chains, with irregular groups, on solid media.

As a standard routine method we recorded the grouping of each culture as shown on the agar streak. The streaks used were never more than three days old, and the grouping was observed after staining lightly with methylene blue and mounting in cedar oil. Too heavy staining may introduce a serious error by making packets 
of small sarcinæ appear like large single cells. The observations on the stained preparations were controlled by careful examinations of the slides prepared for the study of the Gram stain, as noted later.

We distinguished two primary types of morphology, depending upon the presence or absence of packets. In the first group occur the streptococci, which produce pairs, long chains, and irregular groups; and the intermediate micrococci, which show pairs, short chains, fours, and irregular groups: while the sarcinæ include organisms which produce fours, irregular groups, and packets, as well as those extreme forms which show only packets. None of these differences, except the presence or absence of packets, appear on agar with sufficient constancy to be determined definitely. For distinction between streptococci and micrococci the observation of broth cultures would perhaps be valuable; but the appearance of the agar culture to the eye is sufficient to distinguish the former group.

Dimensions. The cocci exhibit a range in size from O.I to $2.0 \mu$, with considerable variation between individual cells in the same culture. We were somewhat surprised to find that we could demonstrate no definite relation between size and the age of cultures, or the conditions of cultivation. In a series of preliminary studies the same organism was grown on seven kinds of media and examined at intervals during a period of two months. The maximum size, in different cultures, was 
recorded on the first, second, seventh, fourteenth, fortysecond days and after two months, respectively. The largest cells appeared indiscriminately in broth at 37 degrees, in broth at 20 degrees, on Nährstoff-Heyden, nutrient gelatin, acid and alkalin gelatin, and under anaerobic conditions. In other words, the age and kind of medium had no constant effect, except that in most cases the Nährstoff and other poor media showed the smallest individuals. These results are not in harmony with those of other observers. Neisser and Lipstein (1903) state that unfavorable conditions, such as excessively high temperatures, and acidity or extreme alkalinity of the medium, tend to produce large cells; and the same thing has been noted in old cultures by other authors. It might naturally be expected that cells would be smaller in rapidly growing cultures than in those in which development had been checked. In our investigations, however, no constant difference in size was apparent in young and old cultures, on solid and in liquid media. One series of organisms examined in dextrose broth and on agar, at periods ranging from one day to two weeks, showed the same average size in both media and at all ages. Finally, we attempted to see whether prolonged cultivation under special conditions would affect the size of the cell. Cultures were grown for ten days in broth at 37 degrees, on nutrient gelatin at 20 degrees, and on acid and anaerobic gelatin, with daily transfers. The size of each culture was 
recorded on the tenth day, after which time each was transferred to gelatin and examined after one day. The results showed no significant differences.

In a comparison of size, as determined by examination of living organisms and of stained preparations, the cells appeared generally somęwhat smaller after staining. This is no doubt partly due to shrinkage in drying and partly to the imperfect definition which makes the unstained specimens appear larger than they really are. Occasionally, when the staining was too heavy, the stained cells appeared larger. In any case the differences are unimportant; and we have used the size of the methylene-blue stained preparation throughout our work.

Staining reactions. Since the cocci, as far as we have examined them, all stain easily with methylene-blue, we made no special tests with anilin-gentian-violet. The Gram stain was, however, used on all our cultures, since in the genus Diplococcus and in many other groups it has special importance.

The value of this staining method was studied with considerable care by Mr. A. T. Brant, working in the laboratories of the Institute. Mr. Brant found, as other observers have done, that while certain bacteria are constantly Gram-negative, or Gram-positive, others exhibit an intermediate condition, retaining the stain under some circumstances and giving it up under others. He noted, for example, that all cultures of $B$. coli are decolorized 
by one minute's treatment with alcohol, while $B$. megatherium constantly fails to decolorize after three hours. On the other hand, with $B$. fluorescens, B. diphtherice and certain cocci the result is affected by the time of decolorization, as well as by the age of the cultures. Between the fixed points at the extreme, preparations will yield varying results, showing some cells stained and others decolorized. As a rule, a large majority of cells in a given preparation will show one reaction or the other; but a second slide made from the same culture may yield a different result.

The time chosen for decolorization is, of course, an arbitrary factor which will affect the proportion of positive results obtained. In our work, as a result of Mr. Brant's experiments, we fixed on three minutes, altho we are not certain that this is preferable to the fiveminute period recommended by the Committee on Standard Methods. We have applied the anilin-oil-gentianviolet for one and a half minutes and the Grám solution for one and a half minutes, instead of the one- and twominute periods of the committee.

In all cases we made the stain on young 20-degree agar cultures (not over five days old), and in each case the fest was made in duplicate at different times. When the result of the two tests coincided, the culture was recorded as positive or negative. Cultures which gave one positive and one negative test, or in which the stained and decolorized cells appeared in 
about equal proportions, were recorded in an intermediate class.

Flagella. In view of the work of Ellis (I902), we devoted considerable time to the study of motility among the cocci. This author reported the finding of spores and flagella in various streptococci and sarcinæ, and Arthur Meyer has carried the position of the Marburg bacteriologists to an extreme in the statement that "the researches of Ellis have rendered it doubtful whether there are any species of bacteria which entirely lack flagella" (Meyer, I903). We examined a number of cultures very carefully, transferring them at frequent intervals on different media, according to the general plan adopted by Ellis. We found in almost every case active vibratory movements, with a tendency to incomplete rotation, the successive jerks sometimes producing a gradual translation across the field; but never true spontaneous motion. This type of behavior is entirely different from the motility, characterized by slow, steady revolution, which appears in such forms as the $M$. agilis of Ali-Cohen. We are convinced that most of the cocci are non-motile, while a few forms show true movement, associated with clearly stainable flagella. The study of this character is therefore of significance. It is questionable, however, whether it is not one of the less important characters in this group of bacteria. It appears, from the published descriptions of species, that this property is not correlated with any other character, - arising 
independently in forms exactly resembling - non-motile forms in every other respect. On account of its rarity and this apparent lack of correlation with other differences, the property of motility was omitted in our work.

Spores. The experiments carried out by Ellis (I902) strongly suggest the presence of specially resistant cells in old cultures of the cocci. His figures are, however, by no means conclusive as to the existence of true spores. In the absence of any observations as to germination, we have not felt that the evidence warranted extensive study of this character.

Fission. A study of the conditions influencing the growth-forms of the Coccaceæ might be of considerable interest. Pairs and chains are apparently associated with meager, and groups and packets with more abundant, development. The effect of the general rate of growth must, however, be modified by the rate at which cell-wall and cell-protoplasm, respectively, are formed. A careful study of the method by which groupings arise in cell-division, such as could be made by the use of Hill's hanging-block method, might throw much light on all such points. In the present study, however, we limited ourselves to the observations made on stained preparations from ordinary cultures.

Capsules. Considerable preliminary work failed to indicate any constant differences in capsule formation among the cocci studied. This character appears to be of considerablè value among the diplococci (Buerger, 
I904); but even with them it varies markedly with the medium used for cultivation. We cultivated certain selected organisms in broth at 20 degrees and at 37 degrees, on nutrient gelatin, acid gelatin, alkalin gelatin, anaerobic gelatin, and Nährstoff-Heyden agar; and examined them at intervals by Welch's staining method. In every case capsules were apparent at some stages, being most strongly developed in old cultures and on poor media like the Nährstoff agar. This character has not seemed to us of sufficiently promising diagnostic value to be included in routine examinations outside the genus, Diplococcus.

Involution and degeneration forms. In numerous examinations of old cultures we found no involution forms of special significance. As noted above, swollen and oval cells are more apt to occur in old cultures of cocci, but they are not sufficiently definite to warrant record.

\section{Cultural Characteristics.}

In this study we necessarily included only those tests which reveal definite and independent variable characters. Most of the commonly observed cultural characteristics are the secondary results of a few fundamental properties, which can be observed on one medium as well as on several. For this reason we have eliminated a number of the ordinary media from our routine. The general character of the growth is approximately the same on agar, blood serum, potato, or Nährstoff, 
except that agar always has markedly more growth, and potato often none. An organism producing abundant chromogenic growth on agar will give good growth and some pigment on the other media. The streptococci on the other hand form a restricted and veil-like growth on serum and Nährstoff, and usually no growth on potato. In other words, with the organisms studied by us, Nährstoff agar, serum, and potato showed no specific characteristics other than those due to feebleness of growth. Blood serum may be useful in other groups to show a special type of liquefaction, but in a preliminary study of 50 of our cultures we never found this to occur; and it has been very rarely and doubtfully recorded in published descriptions of the Coccaceæ. In twenty-five out of fifty cultures inoculated on potato no growth occurred, and in no case have we observed discoloration. These media have therefore been omitted; and the action is in accordance with the conclusions of the Committee on Standard Methods (I905) in considering their value for general diagnostic use.

Nutrient broth. In the group of the cocci we have not found that any information of definite value could be derived from a study of broth cultures. None of the cultures examined form a surface pellicle or produce any characteristic odor. There remain to be observed only two features - turbidity and sediment - which, in our judgment, depend directly on other properties, such as the general vigor of growth and the size of the cell 
aggregates. Both turbidity and sediment vary markedly with the age of the culture; what is first turbidity later settles to form sediment. The amount of either depends on the activity of growth. A constant difference often appears between cultures which early in the course of development show considerable turbidity with little or no sediment; and those which almost at once develop a heavy sediment with colony-like masses of growth clinging to the walls of the tube. This difference, however, appears to be correlated with the growthform and general vigor of the coccus. Organisms of the Streptococcus type with cells separating readily, which show faint surface growth, produce chiefly turbidity; while organisms like Sarcina, with large cell aggregates and rich surface growths, show heavy sediment.

Gelatin plates. Minute differences in the macroscopic and microscopic appearance of colonies on gelatin are given great weight in German systems of classification. Certain special characteristics do, indeed, appear in old gelatin colonies of the cocci after several weeks of incubation. Colonies may remain almost spherical, or they may expand in flat, disk-like growths with terraced edges. Sometimes a distinct boss appears at the center, surrounded by a flatter area. The edges may be entire, or more or less deeply scalloped, and the edges of the scallops may be produced inward in folds. Concentric rings sometimes appear in the interior of the colony, or zones of partially liquefied gelatin around its periphery. 
Some of these characters vary, without any apparent reason, as different colonies on a plate show different characteristics; this is perhaps due to the depth at which the original cell lay below the surface of the gelatin. Most of them are profoundly modified by variations in the amount of moisture in the gelatin, and in the atmosphere above. In a series of comparative studies with different conditions of incubation we found that highly characteristic colonies of granular structure, with deeply lobed edges and indented surfaces, could be produced by cultivation in an incubator whose atmosphere was kept dry by calcium chloride. Dunham (I903) has pointed out the wide differences which may be due to slight variations in the physical properties of the gelatin used. In some groups of bacteria, the Proteus group and the $B$. ramosus group for example, colony forms are obviously characteristic. Among the cocci, however, a slightly elevated disk appears to be the prevailing form of colony under favorable conditions of moisture and with the proper consistency of gelatin. In our experience, all the constant differences observed could be explained by variations in general vigor of growth and in rate of gelatin liquefaction. These two factors, liquefying power and general vigor of growth, were observed on the gelatin stab and the agar streak, respectively.

Gelatin tubes. All our cultures have been studied in the gelatin tube, and the rate of liquefaction has been 
systematically recorded. The only significant differences between various non-liquefying colonies lie in the amount of surface growth and the color, both of which characters are more easily studied on the agar streak. The nature of the surface growth, like that of the gelatin plate colony, does not.appear in this group to offer any character of diagnostic value, and all the cocci grow fairly well in the stab. Among the liquefying forms we have not found the shape of the liquefaction of sufficient constancy to be recorded. Whipple (I902) has shown the uncertainty of this character - almost every possible type appearing in media made with slightly different commercial gelatins. The Committee on Standard Methods (1905) has also omitted this property.

The amount of liquefaction of gelatin was therefore the only character recorded in the gelatin tube. The method by which this was measured will be described under "Biochemical Reactions."

Agar plates. The same reasons which led us to omit the gelatin plate militate against the use of the agar plate as a diagnostic test. Constant differences between colonies are slight and depend on a few fundamental properties which may be more easily observed on other media, notably on the agar streak.

Agar tubes. The general conclusion from what has been said in this discussion of cultural characteristics is that in the cocci a single medium is sufficient for their 
determination. We should, of course, deprecate any extension of this conclusion to other groups where the gelatin stab or the plate culture may yield information of definite value. In the absence of evidence as to the significance of agar and gelatin plates, potato cultures, etc., it seems unwise to repeat tests mechanically and without any definite purpose, merely because they have had an important place in the historical development of the science.

Therefore, in our work, all cultural characteristics were observed in the agar tube. A combined streak and stab was made on a slant surface, and the cultures were uniformly studied after incubation for two weeks at 20 degrees centigrade. Cultures of different age exhibit marked differences, but the characters of the old culture are the outcome of those of the new. Comparative studies with lactose agar and glycerin agar showed neither to be as favorable a medium as ordinary nutrient agar.

In order to obtain a comparative idea of cultural characters, we examined agar streak cultures, two weeks old, made from the whole 500 strains at the same time. The visible differences between the cultures were due almost wholly to two properties, - chromogenesis and general vigor of surface growth. There was a distinction in luster between a large majority of the cultures which had smooth and shining surfaces and a few which were dull and rough. This difference appears, however, to be due mainly to the relative amount of growth 
and moisture in the tube. Faint growths are moist and shining, while heavy growths in tubes which do not contain much moisture show the dry, rough, dull appearance. The "white chromogens" showed another difference, varying from an opaque porcelain-white to a duller and more translucent growth, of indefinite color and somewhat shiny appearance. The latter type of growth was distinctly viscid to the needle.

We noted, as cultural characters on the agar streak, the color production and the vigor of surface growth. The method of studying the former character will be described under "Chromogenesis." Under "Vigor of Surface Growth" we distinguished five different types. Grade I includes forms like the Streptococcus which form only a very faint, veil-like growth or a few translucent dotted colonies on the surface. Grade 2 is reserved for a somewhat more abundant, but still meager growth. Grade 3 corresponds to a good but not abundant streak; Grade 4 to an abundant growth; and Grade 5 to a very heavy surface development.

The response to free oxygen is directly related to the vigor of surface growth; the streptococci, which form only a faint film on the surface, grow fairly well in the stab; and conversely the sarcinæ, which form abundant surface masses, grow badly in the stab. The agar streak and stab culture, therefore, distinguish in a general way the semi-anaerobic from the strongly aerobic forms. Inhibition of growth by acidity and alkalinity of media, 
temperature relations, and pigment formation were also recorded on this medium under conditions to be described below.

\section{Biochemical Reactions.}

Action upon milk. Milk is a favorable nutrient medium for bacterial growth because of its rich food properties, and in many groups its reactions are important. It has, however, no specific diagnostic value for the Coccaceæ, since all the changes it undergoes are correlated with those which occur in the sugar broths, and with the general activity of the organism. No coagulating enzymes and no casein-digesting enzymes have been observed in any of our cultures and we have never seen gas formation in a pure culture of cocci. These various reactions have at times been recorded by individual observers. Thus Dyar (I895) described a coccus which coagulated milk in alkalin solution. Leichmann (I896) reports the isolation of a coccus which, when grown in milk, formed carbonic acid gas and levo-rotary lactic acid. The great weight of negative evidence on the other side makes it probable that these observations were due to errors of technique, and makes it quite certain that such properties are at least exceptionally rare. As a rule the only changes which the cocci effect in milk are the production of acid or alkali, coagulation, and decolorization of the litmus.

Decolorization has no significance, except that it indicates general activity of the organism. When a 
bacterium is most-active, it uses up oxygen and reduces the litmus, which is accordingly decolorized; and, conversely, when activity grows less, oxygen diffuses down from the surface, making the litmus pink again.

Coagulation usually depends' upon the amount of acid produced, and is more accurately and easily studied in sugar broth cultures.

Action upon carbohydrates. The characteristics usually observed in sugar broth are turbidity and sediment, relation to oxygen, gas production, and acid production. We have given reasons, in discussing nutrient broth, for considering turbidity and sediment unimportant; and the relation to oxygen is most sharply defined by surface growth in the agar tube. None of the cocci that we studied produced gas, and therefore acid production only was recorded. For this purpose ordinary straight tubes were used; and the sugars tested were dextrose and lactose. A preliminary test indicated that saccharose is less commonly fermented by the cocci than are dextrose and lactose.

The media were made up in the usual manner, with 2 per cent of the sugar to be tested. The reaction was made neutral, and after tubing and sterilization it was between 0.5 and $1 . \circ$ per cent. After standing for two weeks sterile blanks showed a slight further rise in acidity; so control tubes were always kept with each batch inoculated and titrated at the end of the 
experiment. After considerable preliminary experimentation, it was decided to titrate with phenolphthalein as an indicator, in the cold. Methyl orange is not sensitive to the organic acids and gives a poor end-point. With phenolphthalein a comparative series of titrations, made on the same tubes, first cold and then boiling, showed slightly higher results by the latter method. Evidently heating increases the apparent acidity by the breaking up of unstable compounds more than it decreases it by driving off carbon dioxid. The cold method was therefore used. To 5 c.c. of the sugar broth, incubated for two weeks at 20 degrees, were added 95 c.c. of distilled water and two or three drops of phenolphthalein. This was titrated against $\frac{\mathrm{N}}{20} \mathrm{NaOH}$, and from the value obtained was subtracted the acidity of blank controls titrated at the same time. All tests were made in duplicate, and the final value recorded as the acid or alkali production of the organism is the difference between the average of two titrations of tubes in which it had grown for two weeks and the average of two blank controls. No determination was made of the rate of acid production, as distinguished from this total final acidity, tho such observations might be of much interest.

We did not attempt a study of the specific products formed, altho their nature might yield important assistance in distinguishing various species now confused. For example, Nencki (I89I) showed that the production 
of isomeric compounds of different rotating power was characteristic of certain bacteria.

Action upon nitrates. Data with regard to the reduction of nitrates by the cocci are extremely meager, the presence or absence of this character being recorded in very few of the published descriptions. It seems, however, to have a fair degree of definiteness, and we have included it as a qualitative test in our routine. Each organism was inoculated into a series of ten tubes of standard nitrate solution. After seven days' growth at 20 degrees the tubes were tested for nitrites and ammonia in the regular way prescribed by the Committee on Standard Methods (I905). The test for nitrates was omitted after it was found that all the cultures, out of a considerable series tested, gave positive results, without exception.

Production of indol. A preliminary study of some fifty cultures showed no production of indol in any case, and an examination of the literature of the cocci indicates that this property is very rare, if it ever occurs, in this group. It was therefore omitted from our routine.

Inhibition of growth by acidity and alkalinity of media. This character is of considerable importance and warrants careful study, but it is obviously a difficult property to observe in a large series of cultures, and we have not attempted to use it in the present investigation. A preliminary examination of 33 cultures, the results of which are shown in the table below, indicated that I 
per cent is the optimum acidity for a majority of these organisms, and that an excess of acidity over this amount is more generally fatal than an alkalin reaction.

NUMBER OF ORGANISMS SHOWING MAXIMUM GROWTH AND DEEPEST COLOR AT VARIOUS DEGREES OF ACIDITY.

33 Cultures of Cocci.

\begin{tabular}{|c|c|c|c|c|c|c|c|}
\hline Per Cent of Acidity. & -1.0 & -.5 & $\circ$ & +.5 & +1.0 & +1.5 & +2.0 \\
\hline Growth & 2 & 4 & 5 & 6 & 9 & 6 & I \\
\hline Color. & 3 & I & 0 & 3 & 9 & 6 & 3 \\
\hline
\end{tabular}

8 organisms showed no color.

Relation to free oxygen. The Committee on Standard Methods (I905) recommends that the relation of bacteria to oxygen be studied by the comparison of cultures made under normal and under anaerobic conditions. A preliminary study of fifty cultures, made in this way, led to the belief that such a procedure is unnecessary among the cocci. It became evident that there are two main types of these organisms: those which, like Streptococcus, grow only feebly on the surface of aerobic agar and which grow equally well under anaerobic conditions, and those, like Sarcina, which form abundant surface growths under aerobic conditions, and under anaerobic conditions grow feebly like streptococci. In other words, there is little difference between the anaerobic cultures of the cocci. Some forms which grow distinctly, but faintly, without air grow no better with air, while others which almost wholly fail 
to grow without air give abundant aerobic growths. Therefore, for purposes of classification we have considered the study of the aerobic surface growth a sufficient measure of the relation to free oxygen, as well as of general vigor. The five grades of surface growth correspond to five grades of aerobiosis, including all variations from forms which are anaerobic and facultatively aerobic to forms which should be classed as strong aerobes.

Temperature relations. There are two points of special importance which ought to be determined in studying temperature relations - the optimum temperature and the high death-point. The death-point at extreme low temperatures is too indefinite to be attempted, and the extreme limits of growth may be omitted as far less important than the other two properties.

For the determination of the optimum temperature we first made a series of preliminary studies by comparing agar cultures grown at Io, 20, 37, 45, and 56 degrees. Two cultures grew better at 20 degrees, 18 developed equally well at 20 and 37 degrees, 22 showed an optimum at 37 degrees, two grew equally well at 37 and 45 degrees, and four grew best at 45 degrees. These conclusions refer only to the amount of growth,- color production being generally most active at 20 degrees. From these results we concluded that the information to be gained by cultures grown below 20 degrees and above 37 degrees would be scarcely commensurate with 
the labor involved, and we have limited our observations on optimum temperature to the comparison of growth and color production at 20 and 37 degrees. Cultures were grown for this purpose on agar for two weeks and compared by inspection. Amount of growth and depth of color were recorded in five arbitrary grades according as growth or color production was much better at 20 degrees, somewhat better at 20 degrees, equal at the two temperatures, somewhat better at 37 degrees, or much better at 37 degrees.

Thermal death-points were included in the original plan of our experiments and observations were made on $87 \mathrm{cul}$ tures. The process used was to inoculate, from 3- to 5-dayold agar cultures, into broth tubes brought to the desired temperature in a water bath heated electrically by a platinum coil, and to expose them for ten minutes. The tubes were then cooled and incubated at 37 degrees for six days. At the end of that time, streaks were inoculated from the broth tubes in order to make sure by characteristic growth that the organisms originally inoculated were present. Tests were made from $5 \circ$ degrees up to the point where growth failed. The general results obtained are as follows:

THERMAL DEATH-POINTS. 87 CULTURES OF COCCI.

Number of Cultures Killed at Various Temperatures.

\begin{tabular}{l|c|c|c|c|c|c|c}
\hline Temperature....... & $50^{\circ}$ & $55^{\circ}$ & $60^{\circ}$ & $65^{\circ}$ & $70^{\circ}$ & $75^{\circ}$ & $80^{\circ}$ \\
Cultures ......... & 2 & 5 & 24 & 17 & 16 & 22 & 1 \\
\hline
\end{tabular}


Pigment formation. The production of color by the bacteria is not only markedly affected by contemporaneous conditions of cultivation, but may be profoundly modified by selective action or by the effect of antecedent environment. Among the conditions which temporarily affect the production of color, without modifying the inherent chromogenic power of the organism, may be mentioned the medium, the presence of free oxygen, and the temperature. With some bacteria, media of low nutritive value, like potato and Nährstoff, appear to favor pigment formation, but with the cocci this is not generally the case. Agar has, on the whole, shown a better development of chromogenesis than any other medium tested. The presence of free oxygen is generally an essential for color production, stab growths being almost invariably lightly colored. We found a single exception to this rule in a coccus which produces a reddish pigment of much deeper tint in the stab than on the surface. In comparing color at different temperatures we found, in general, a much better pigment formation at 20 degrees than at 37 degrees. A deep orange growth at the lower temperature may correspond to a white one at 37 degrees. The relative color production at 20 and 37 degrees was recorded in our routine studies, and will be more fully discussed later. Besides these temporary modifications of the chromogenic power, the actual color of cultures may be indirectly affected by certain other factors. The general vigor of growth 
is naturally correlated with depth of color, and the dryness of the atmosphere increases color intensity by evaporating moisture and concentrating the pigment. Both these factors, - increase in the total amount of pigment and concentration by evaporation,- - produce a progressive deepening of color in old cultures.

Even if the temporary conditions of cultivation are quite constant, the chromogenic power of an organism may be modified by its previous history. In thermal death-point observations we have found interesting cases of this sort. Streaks made from broth cultures which had been exposed to a temperature of 50 or 55 degrees were in a few instances deeper in color than was normal for the organism; but in most cases they were much lighter. Sometimes streaks made from a yellow or an orange chromogen after such treatment were almost colorless, altho successive transfers generally restored the normal properties. Finally we have noticed in our work apparent spontaneous variations in chromogenesis such as have been recorded by Neumann (1897), Conn (1900), and Sullivan (1905). The latter authors note that on a plate sown from a single colony there may develop colonies varying appreciably in shade, from which selection of the extremes will produce quite distinct types. Neumann records the sudden appearance of widely different strains, as sectors in old and carefully sealed stab cultures. We have observed both phenomena in our cultures, and are inclined to 
attribute the first, at least, to true variation rather than to contamination.

In spite of all these facts, it is clear that, as the cocci normally occur in nature, chromogenesis is one of their most distinct and significant. differences. In any series of plates sown with washings from the outer skin, three well-marked types - yellow, orange, and white - are fairly certain to occur; and a fourth, red type is common in air plates. Variations due to past and present environment are, of course, easily excluded by the maintenance of constant conditions. Our stock cultures were in all cases kept on agar at 20 degrees, and cultures for chromogenesis were grown on that medium, and at that temperature, for two weeks. In order to avoid the apparent differences due to vigor of growth or to evaporation a fixed portion of the growth was removed on a loop needle and spread evenly on white drawing-paper with a rough surface. After drying at the room temperature, the color was compared with an arbitrary standard scheme.

The color chart used for matching these colors was devised after a very careful study of the colors actually found among the Coccaceæ, and includes nine hues, ranging from white through lemon-yellow, light cadmium, medium cadmium, lemon-yellow and cadmium orange, cadmium orange, red and cadmium orange, to two other shades of red. We have used, under each hue, nine different chromas, obtained by successive washes 
of the hues on white paper. This color chart is reproduced as the frontispiece of the book. The nine hues, passing from left to right, we have designated as White, Light Lemon Yellow, Light Cadmium Yellow, Medium Cadmium Yellow, Orange Yellow, Cadmium Orange, Orange Red, Medium Red, Dark Red. The chroma is indicated by a number which corresponds to the number of washes of the hue applied, ranging from I to IX.

We did not make a study of the pigments formed, from the chemical standpoint, altho this subject holds out promise of significant results. Schneider (I897) made many observations upon the solubility of bacterial pigments in various liquids, and upon the effect of acids, alkalies, etc., upon the solutions obtained. The results are cited in considerable detail by Migula (I900) in his systematic account of the particular organisms studied. The results are sufficiently suggestive to make further work along this line eminently desirable.

Liquefaction of gelatin. The liquefaction of gelatin, like the property of pigment production, is subject to considerable variations. Kruse (1896), for example, found that staphylococci lose their liquefying power after prolonged cultivation under anaerobic conditions. Spontaneous variations, too, may occur under approximately uniform environmental conditions. Conn (I900) was able by selection to obtain from a single culture of a milk coccus two extreme variants, one a rapidly lique- 
fying form and the other with almost no peptonizing power. Smith (I900) records a similar experience with colon-bacilli and forms of $B$. proteus.

In studying liquefaction we determined only the extent of the action exerted. The-shape of the liquefaction in the stab culture has been shown by Whipple (I902) to vary within the widest limits, with slight differences in the character of the medium; and the Committee on Standard Methods (I905) has omitted this character from its list.

For determining the amount of liquefaction we used the method suggested by Clark and Gage (1905), which consists in inoculating gelatin tubes of Io millimeters diameter, by spreading a suspension of the organism over the surface. Liquefaction proceeds in a stratiform fashion, and its amount may be read off in centimeters. With such a method one may determine the rapidity of liquefaction either by the number of days required to reach a fixed point or by the final amount of liquefaction. In general, these two values are pretty closely correlated, but in a preliminary study we found that the final differences after a fixed period were somewhat sharper as well as easier to record. We therefore adopted the depth of liquefaction after 30 days as our routine measure of liquefying power.

Supplementary tests. Many other tests than those mentioned are sometimes used in bacterial diagnosis, but none of them seemed suited to the present investi- 
gation. Serum reactions, in spite of their variability, throw considerable light on the mutual relationships of the parasitic cocci; and the numerous investigations which have been carried out by students of the diplococci and the streptococci will be discussed in Chapters $\mathrm{V}$ and VII. In some groups, notably among the white and orange staphylococci of the skin, further comparative study of agglutinative reactions in relation to various biochemical powers should prove of value.

Among other measures of bacterial activity the test for the liquefaction of starch is one which it seems logical to include with those which show the relation of an organism to gelatin and the sugars; and we, therefore, made some experiments with the starch media introduced by Smith (I905). It appeared that certain cocci did exert an amylolytic action; and the further study of this character would probably prove of considerable interest.

Those characters which we finally chose as most likely to throw light on the systematic relationships of the Coccaceæ are as follows: (Each of the tests was applied under comparable conditions to the series of 500 cocci at our disposal.)

DIAGNOSTIC TESTS USED FOR STATISTICAL STUDY OF THE COCCI.

I. Habitat. Recorded as I (diseased conditions); 2 (normal body); 3 (water); 4 (earth); or 5 (air). The significance of these various habitats has been more 
fully discussed above. It should be noted, however, that group 5 includes certain laboratory cultures whose origin was unknown.

2. Grouping of cells and dimensions. Observed in stained preparations, made from 20-degree agar cultures less than five days old. Grouping recorded as I (packets present), or 2 (packets not present). Extreme dimensions recorded in micro-millimeters to the nearest tenth.

3. Relation to Gram stain. Observed on two different occasions; on 20-degrees agar cultures less than five days old. Treated with anilin-oil-gentian-violet for $\mathrm{I} \frac{1}{2} \mathrm{~min}$ utes; Gram's solution, I $\frac{1}{2}$ minutes; 95 per cent alcohol, 3 minutes. Counterstained with Bismarck brown for one-half minute. Reaction recorded as - (decolorized in both tests); \pm (stained once and decolorized once); or + (stained in both tests).

4. Vigor of surface growth on agar streak after I4 days at 20 degrees. Recorded as I (very faint); 2 (meager); 3 (good); 4 (abundant); or 5 (very heavy).

5. Amount of acid produced in 2 per cent dextrose broth after $\mathrm{I} 4$ days at 20 degrees. Determined by titration against $\frac{\mathrm{N}}{20} \mathrm{NaOH}$ in the cold with phenolphthalein as an indicator. Recorded value is the difference between inoculated tubes and sterile controls, expressed as per cent of normal. 
6. Amount of acid produced in 2 per cent lactose broth. Same conditions as under 5 .

7. Formation of nitrites in nitrate solution. Observed in a series of 10 tubes grown for 7 days at 20 degrees.

8. Formation of free ammonia in nitrate solution. Same method as under 7 .

9. Comparative growth and color production, after I4 days on agar streak at 20 degrees and 37 degrees respectively. Recorded as I (much more vigorous at 20 degrees); 2 (more vigorous at 20 degrees); 3 (equal); 4 (more vigorous at 37 degrees); or 5 (much more vigorous at 37 degrees).

I0. Chromogenesis. Hue and chroma of pigment produced on agar at 20 degrees after I4 days, determined by comparison with color scheme as described above.

II. Depth in $\mathrm{cm}$. of gelatin liquefaction in tube of Io $\mathrm{mm}$. diameter after 30 days at 20 degrees.

It would be well to extend this series of tests by study of the cell-grouping in broth, motility, fission on the agar block, fermentation of saccharose, effect of acid and alkalin media, and the thermal death-point. Data in regard to agglutination reactions might throw light on the systematic relations of many members of the group. Investigations as to the power of cocci to break up raffinose, inulin, mannite, dulcite, and many other carbohydrate media have proved of significance in the hands of Gordon, Houston, Andrewes and Horder, and 
other students of the diplococci and streptococci. The chemical constitution of the pigments formed, the power to liberate ammonia from urea, the ability to dissolve starch and many other biochemical properties must be measured and compared before the group of the Coccaceæ can be adequately understood. The eleven tests listed above have, however, furnished, as it seems to us, sufficient information to warrant the recognition of the most important natural groups. 


\section{CHAPTER IV.}

\section{SUBFAMILIES AND GENERA OF THE COCCACEA.}

THE application of the tests enumerated in the last chapter, to five hundred cultures of cocci, gave eleven more or less definite facts in regard to each culture. The records were then analyzed to find the centers of variation for each character and to determine what characters were mutually correlated. The detailed analysis of the figures has been published elsewhere (Winslow and Rogers, I906), and need not be repeated here.

The first result of a study of the correlated characters of the five hundred cultures was the conclusion that the division of the Coccaceæ into a parasitic and a saprophytic subfamily is amply justified, altho the characters by which we originally defined these subfamilies require modification and extension. Of the 500 strains studied, 59 had been isolated from diseased conditions and 170 from the normal human body, making a total of 229 from a parasitic habitat; 95 were from water, 67 from earth, and Iog from air, a total of $27 \mathrm{I}$ from saprophytic environments. Obviously such a distinction cannot be absolute. Cocci get on the skin and into the alimentary tract from various sources; and parasitic forms occur in water, 
earth, and air. On the whole, however, the average characters of the two groups are quite distinct.

In almost every point tested the group of 229 cocci from the body differed in its average characters from the group of $27 \mathrm{I}$ cocci isolated from other sources. In the first group II per cent were uniformly Gram-negative, 29 per cent variable, and 60 per cent uniformly Grampositive; in the second group 44 per cent were uniformly Gram-negative, 29 per cent variable, and 27 per cent uniformly Gram-positive. Of the parasitic cultures, I4 per cent formed a faint or meager growth on agar, 53 per cent a good growth, and 33 per cent an abundant or very heavy growth; of the saprophytic forms only 3 per cent formed a faint or meager growth, 35 per cent a good growth, and 64 per cent an abundant or very heavy growth. Of the parasitic cultures, only 28 per cent produced an alkalin or faintly acid reaction (.003 normal) in dextrose broth, 44 per cent produced an acidity between .003 and .007 normal, and 28 per cent an acidity of .007 normal or over; among the saprophytes $5^{8}$ per cent produced a reaction, alkalin or less than .003 normal, and 20 per cent an acidity between .003 and .007 normal. For lactose broth the corresponding figures were 34 per cent, 34 per cent, and 32 per cent for the parasites, against 65,22 , and 13 per cent for the saprophytes. In nitrate reduction there was no well-marked difference between the cocci from the body and from other sources. Certain generic differences existed in 
this respect, but they were insufficient to affect the averages for the larger groups. In regard to optimum temperature a slight difference was manifest. In both groups a large majority of the strains grew equally at 20 and 37 degrees. Among the cocci from the body, however, 9 per cent grew better at 20 degrees and 27 per cent better at 37 degrees; of the saprophytic cocci, 23 per cent grew better at 20 degrees and only 8 per cent at 37 degrees. In the proportion of gelatin liquefiers the cocci from the body did not differ as a whole from those found in saprophytic environments. In the rate of action manifested by the liquefying forms there was, however, a distinct difference. Of the cocci from the body, 35 per cent failed to liquefy, 26 per cent produced in 30 days a liquefaction less than $\mathrm{r} .5$ centimeters deep, and 39 per cent a liquefaction deeper than I. 5 centimeters; of the cocci from other sources, 39 per cent failed to liquefy, 43 per cent produced a liquefaction less than $\mathrm{x} .5$ centimeters deep, and only I 8 per cent a liquefaction deeper than $\mathrm{r} .5$ centimeters.

One of the most striking differences between the parasitic and saprophytic forms appeared where it was least expected, - in the respective chromogenic power of the organisms. The common opinion among bacteriologists has been that differences in pigment production were of comparatively slight importance. The yellow and white and red forms were indeed separated by Migula and Chester; but no distinction was made by either author 
between the lemon yellow of such types as M. luteus and the orange of $M$. aurantiacus (Schröter-Cohn). On the other hand, medical bacteriologists have recognized white, yellow, and orange forms among the staphylococci of the skin; but it has been maintained by competent investigators - that these differences were scarcely of even varietal rank.

A study of the distribution of our five hundred cultures in regard to pigment production showed, however, that the four common types of chromogenesis represent real centers of variation. Chromogenesis was studied, as described in the preceding chapter, by growing each culture for two weeks on agar at 20 degrees, spreading a portion of the growth on white paper, drying it and then matching against the color chart reproduced as the frontispiece of this volume. This chart includes nine different hues, one white, three yellows, two oranges, and three reds, with nine different chromas, or depths of color, under each hue. The chroma number indicates in each case the number of washes of the pure color used to produce the effect reproduced in the figure. In the figure each vertical column represents one hue, and the numerical values at the side correspond to the chromas. The transparent sheet, which may be superimposed over the frontispiece, shows the distribution of the five hundred cocci studied according to their pigment-producing power. The general habitat of each organism is indicated by the symbol used, a cross standing for a strain isolated from the body, and a circle for one of saprophytic origin. 
The first point made evident by this diagram is the fact that the yellow and orange pigment producers are not intergrading forms but separate and distinct types. There is a well-marked center of variation in the lighter chromas of cadmium yellow, and another in the darker chromas of cadmium orange, with a much smaller number of cultures showing colors of intermediate grade. The second point which will be noticed is the marked correlation between chromogenic power and habitat. It is evident that four groups of cocci are represented on the chart, corresponding to the four types of pigment production familiar to any student of the cocci. In the upper left portion of the diagram are white (or colorless) parasitic forms. Both the feebly growing streptococci and the more vigorous white staphylococci are included under this head. In the yellow, another sharply marked center of variation appears, this time including organisms mainly of saprophytic origin. The orange forms are sharply contrasted, being almost wholly parasitic in origin; and the red pigment producers, in the last three columns of the figure, are with one exception saprophytic.

These relations may be shown in another way by dividing the cocci studied into arbitrary groups according to chromogenesis. For this purpose we have classed the organisms in the White column and in the upper portions of the Light Yellow columns as White (White, I Light Lemon Yellow, I-III,-Light Cadmium Yellow, I-III). The deeper shades of Light Cadmium Yellow, 
all the Medium Cadmium Yellow, and the lighter chromas of Orange Yellow we have called Yellow (Light Cadmium Yellow, IV-IX, - Medium Cadmium Yellow, - Orange Yellow, I-IV). The deeper chromas of Orange Yellow and all the Cadmium Orange column have been called Orange (Orange Yellow, V-IX, - Cadmium Orange). The last three columns have been grouped as Red. It will be noticed that these divisions correspond, as nearly as possible, to the natural divisions between the centers of variation indicated on the plot. Grouping the cultures in this manner the relation between habitat and chromogenesis may be brought out as indicated in the table below. These figures show that the orange cocci are characteristically parasitic forms, while the yellow and red pigment producer's occur primarily in other environments. The case is not so clear in regard to the cocci we have classified as White. We had only 40 cultures in this class and $\mathrm{I}_{3}$ of them were isolated from the air, which unduly increases the saprophytic group. Other considerations will show that the white cocci, like the orange ones, are primarily parasitic.

HABITAT OF COCCI OF VARIOUS CHROMOGENIC CHARACTERISTICS.

\begin{tabular}{|c|c|c|}
\hline \multirow{2}{*}{ Color of Pigment. } & \multicolumn{2}{|c|}{$\begin{array}{c}\text { Percentage of Cultures from } \\
\text { Each Habitat. }\end{array}$} \\
\hline & Parasitic. & Saprophytic.* \\
\hline 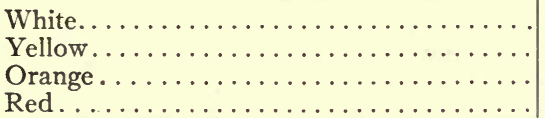 & $\begin{array}{r}53 \\
28 \\
76 \\
4\end{array}$ & $\begin{array}{l}47 \\
72 \\
24 \\
96\end{array}$ \\
\hline
\end{tabular}

* Including air forms. 
The study of other characters correlated with pigment production strengthened the conclusion that the color types of the cocci correspond to four natural groups. In cell grouping, the property of packet formation occurred in about half the yellow and red chromogens studied; it was observed only rarely and doubtfully among the white and orange forms. The reaction to the Gram stain, as shown in the table below, exhibits a similar sharply marked difference.

CORRELATION BETWEEN CHROMOGENESIS AND GRAM STAIN.

Number of cultures in each class.

\begin{tabular}{|c|c|c|c|c|}
\hline Gram. & White. & Yellow. & Orange. & Red. \\
\hline & 6 & I09 & I5 & I5 \\
\hline \pm & 9 & 86 & 46 & 7 \\
+ & 25 & 59 & I 20 & 3 \\
\hline
\end{tabular}

Among the whites and oranges (the parasitic forms) positive Gram reactions predominate, and negative ones are rare. Among the saprophytic yellows and reds, conditions are symmetrically reversed.

CORRELATION BÉTWEEN CHROMOGENESIS AND SURFACE GROWTH.

Number of cultures in each class.

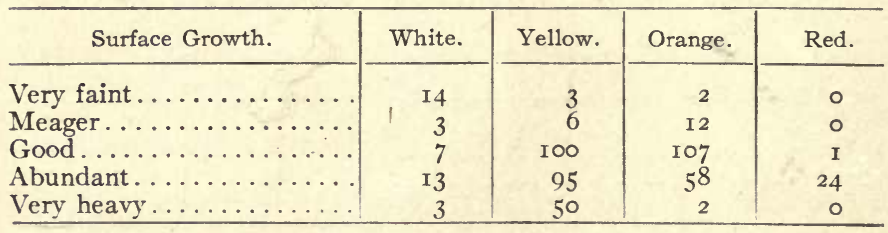


A comparison of the general vigor of growth shows that each color has here again its own relation. Among the white forms, two maxima appear, one under "very faint" growth and one under "abundant" growth. This is because this group is a compound one, including first, forms which give a really white growth, abundant in amount, and second, the feebly growing streptococci which are classed here, altho they really produce no pigment at all. The yellow and orange chromogens show maxima under the "good" growth; and almost all the "very heavy" growths belong to the yellows. The red forms are almost all of one type, - the "abundant."

CORRELATION BETWEEN CHROMOGENESIS AND DEXTROSE FERMENTATION.

Number of cultures in each class.

\begin{tabular}{|c|c|c|c|c|}
\hline $\begin{array}{c}\text { Acid Produced (per cent } \\
\text { Normal). }\end{array}$ & White. & Yellow. & Orange. & Red. \\
\hline$\circ . \circ$ and alkalin.......... & 5 & I I I & 7 & 5 \\
\hline $0 . \mathrm{I}-0.2 \ldots \ldots \ldots \ldots \ldots \ldots$ & 7 & 62 & - 24 & 7 \\
\hline $0.3-0.6 \ldots \ldots \ldots \ldots \ldots$ & 5 & 45 & 92 & 9 \\
\hline $0.7^{-2} .0 \ldots \ldots \ldots \ldots \ldots$ & I5 & 33 & 53 & 3 \\
\hline Over $2.0 . \ldots \ldots$ & 8 & 3 & 5 & I \\
\hline
\end{tabular}

CORRELATION BETWEEN CHROMOGENESIS AND LACTOSE FERMENTATION.

Number of cultures in each class.

\begin{tabular}{|c|c|c|c|c|}
\hline $\begin{array}{l}\text { Acid Produced (per cent } \\
\text { Normal). }\end{array}$ & White. & Yellow. & Orange. & Red. \\
\hline-0.2 and under. ......... & 3 & 33 & 9 & 5 \\
\hline$-0.1-0.0 \ldots \ldots \ldots$ & 8 & I39 & 39 & 16 \\
\hline $0 . \mathrm{I} \rightarrow 0.4 \ldots \ldots \ldots \ldots \ldots \ldots$ & I2 & $6 I$ & 64 & 3 \\
\hline $0.5-1.4 \ldots \ldots \ldots \ldots \ldots \ldots$ & 6 & I7 & 63 & $\mathbf{I}$ \\
\hline I.5 and over $\ldots \ldots \ldots \ldots$ & I I & 4 & 6 & $\circ$ \\
\hline
\end{tabular}


The correlations between chromogenesis and the fermentation of the sugars are singularly perfect. The white forms in each case show two maxima, one corresponding to the true white chromogens, the second, at a higher acidity, to the colorless streptococci. The latter include a majority of the strongest acid-producers in each sugar. The other types show for each sugar a regular and characteristic curve, as indicated for dextrose in Figure II. The yellow forms show a mode at the neutral point. The orange chromogens, on the other hand, produce most commonly an intermediate grade of acidity, 0.3 to 0.6 per cent acidity in dextrose broth, and O.I to 0.4 per cent in lactose broth. The red forms show the same relation as the orange forms toward dextrose, while in lactose broth they resemble the yellow chromogens, producing in most cases no change in reaction.

\section{CORRELATION BETWEEN CHROMOGENESIS AND NITRATE REDUCTION.}

Number of cultures in each class.

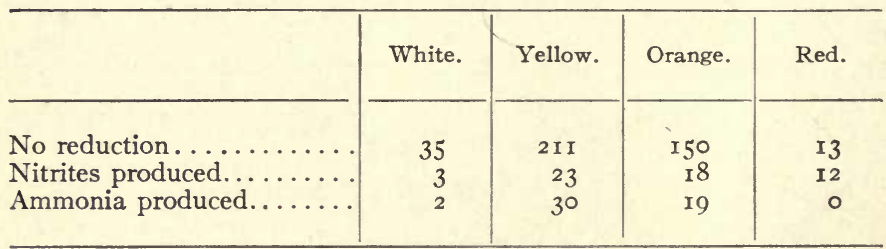

With regard to the reduction of nitrates, the white forms show generally negative results. Nitrites are produced by only one in ten of the yellows, and by a slightly higher fraction of the orange forms, but by half the red- 
pigment-producers. Ammonia production, on the ether hand, appears in one in eight of the yellows, one in ten of the orange forms, and not at all among the reds.

\section{CORRELATION BETWEEN CHROMOGENESIS AND OPTIMUM TEMPERATURE FOR GROWTH.}

Number of cultures in each class.

\begin{tabular}{|c|c|c|c|c|}
\hline Optimum Temperature. & White. & Yellow. & Orange & Red. \\
\hline $\begin{array}{l}20^{\circ} \ldots \ldots \ldots \ldots \ldots \\
20^{\circ} \text { or } 37^{\circ} \ldots \ldots \ldots \ldots \ldots \ldots \\
37^{\circ} \ldots \ldots \ldots \ldots \ldots\end{array}$ & $\begin{array}{r}4 \\
28 \\
8\end{array}$ & $\begin{array}{r}67 \\
155 \\
32\end{array}$ & $\begin{array}{r}13 \\
126 \\
42\end{array}$ & $\begin{array}{r}0 \\
25 \\
0\end{array}$ \\
\hline
\end{tabular}

The majority of forms grow equally at either temperature. Among the white and orange forms, most of those which exhibit any preference grow best at 37 degrees, while among the yellows 20 degrees is more often the optimum. These results accord with the habitats, respectively parasitic and saprophytic, of the two classes.

CORRELATION BETWEEN CHROMOGENESIS AND OPTIMUM TEMPERATURE FOR COLOR PRODUCTION.

Number of cultures in each class.

\begin{tabular}{|c|c|c|c|c|}
\hline Color Production. & White. & Yellow. & Orange. & Red. \\
\hline $\begin{array}{l}\text { Better at } 20^{\circ} \ldots \ldots \ldots \\
\text { Equal at } 20^{\circ} \text { and } 37^{\circ} \text {. }\end{array}$ & $\begin{array}{r}2 \\
3^{8}\end{array}$ & $\begin{array}{r}90 \\
\mathrm{I} 64\end{array}$ & $\begin{array}{r}125 \\
56\end{array}$ & $\begin{array}{r}21 \\
4\end{array}$ \\
\hline
\end{tabular}

It appears from the table that temperature differences affect the production of orange pigment much more than that of yellow; and that the body temperature interferes with red chromogenesis most of all. 
CORRELATION BETWEEN CHROMOGENESIS AND GELATIN LIQUEFACTION.

Number of cultures in each class.

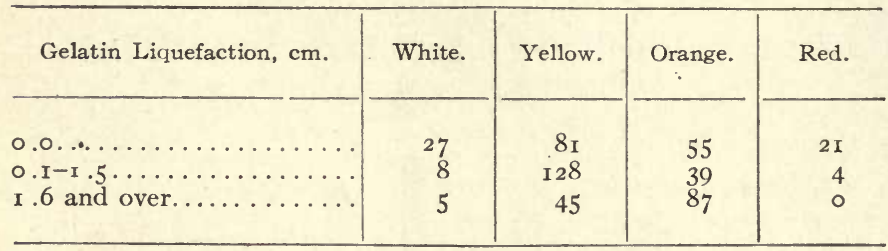

The liquefaction of gelatin presents another correlation with pigment production. The white and red forms are almost all non-liquefiers; the yellow cocci show a maximum among the moderate liquefiers; and the orange chromogens exhibit the peptonizing power to a high degree.

It is clear, therefore, that there exist among the cocci four (or five) distinct groups marked by characteristic pigment production, each group being also defined by a number of other correlated characters. The "white" forms generally stain by Gram, fail to reduce nitrates, grow well at 37 degrees, and usually fail to liquefy gelatin. They include two subgroups - the feebly-growing, strongly acid-producing forms, which are really colorless, not white, and the white-pigment-producers, which grow abundantly and produce a moderate amount of acid. The "yellow" chromogens frequently show packets, are usually Gram-negative, give a good to a very heavy surface growth, produce little or no acid, grow well at 20 degrees, and show a moderate liquefaction of gelatin. The "orange" pigment formers stain well by Gram, 


\section{SUBFAMILIES AND GENERA OF COCCACEÆ 87}

Paracoccaceæ

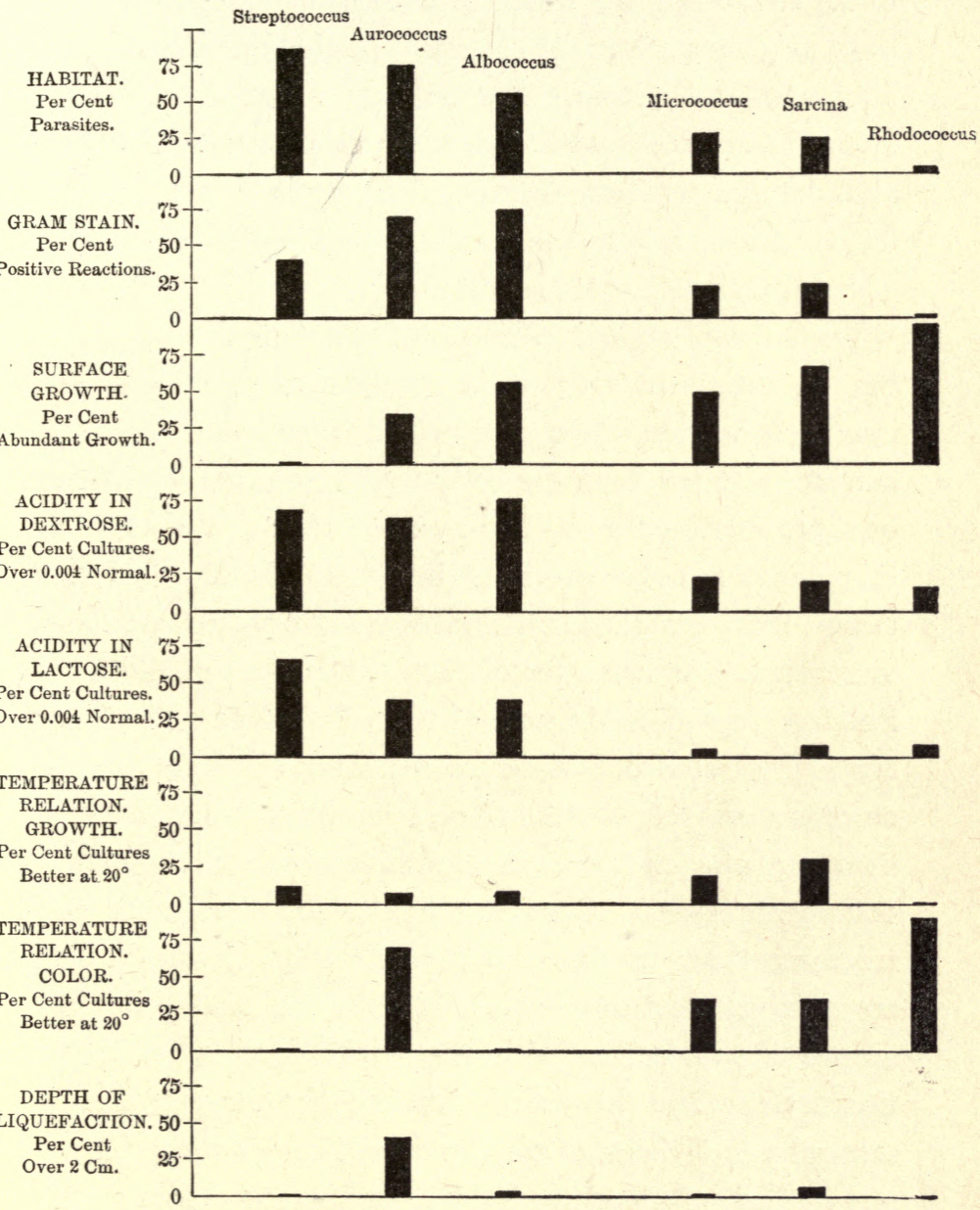

FIG. I. - Characters of Subfamilies and Genera of the Cocacceæ. 
form good surface growths, produce a moderate acidity in sugar broth, grow well, but with poor pigment production, at 37 degrees, and generally produce a considerable liquefaction of gelatin. The red-pigment-producers occur often in packets, are generally Gram-negative, grow abundantly, ferment dextrose slightly but not lactose, form nitrites, but not ammonia, in nitrate solution, and generally fail to liquefy gelatin.

With these general relations of the four color-types of the Coccaceæ in view, it is possible to interpret more intelligently the differences which appeared when the cultures isolated from the body were compared with those of saprophytic origin. The average results cited early in this chapter were naturally imperfect because cultures taken from the skin include stray saprophytic cocci and vice versa. As a matter of fact $3^{I}$ per cent of the cocci isolated from the skin were of the yellow type and 16 per cent of those from water, earth, and air were orange chromogens. On the whole, however, the average character of each flora was determined by its predominant component types. Grouping similar forms together and referring them to the respective habitats in which they occur in greatest numbers, it is clear that the white and orange forms belong with the streptococci as parasitic Coccacex, while the yellow and red chromogens form a saprophytic division of the family. The common characters in each of these two habitat-groups are so many, and the mutual contrast between them so marked, as to leave 
little doubt of the real importance of the subfamilies, Paracoccaceæ and Metacoccaceæ.

In our first paper, to which reference has been made in Chapter II, we included under the Paracoccaceæ only the genera Diplococcus and Streptococcus; and characterized the subfamily by parasitic habit, meager growth on media, and grouping of cells in pairs or chains. It is clear, in the light of the comparative study just reviewed, that the group of the Paracoccaceæ must be broadened to include other characteristic parasitic cocci which resemble the streptococci in important biochemical characters. The new forms to be included are the white and orange staphylococci. Both resemble the streptococci in forming relatively small cell aggregates and never producing packets; but they occur in plates and groups rather than chains. On the other hand, the new facts accumulated make it possible to separate the subfamilies of the Coccaceæ more sharply than before in other ways. Streptococci, white staphylococci and orange staphylococci are alike differentiated from the yellow and red saprophytes by their generally positive Gram reaction, by their marked acid production, and by their peculiar chromogenic power (growth white or orange, not yellow or red). These three characters, with the parasitic habitat, the meager or only moderately vigorous surface growths, and the small cell aggregates, clearly mark off a natural group; and the Metacoccaceæ, including the yellow and red color-types, differ from the 
Paracoccaceæ in all of these respects. We have therefore redefined the two subfamilies as follows (Winslow and Rogers, I906):

Subfamily Paracoccace a (Winslow and Rogers). Parasites (thriving only, - or best, - on, or in, the plant and animal body). Thrive well under anaerobic conditions. Many forms fail to grow on artificial media; none produce very abundant surface growths. Planes of fission often parallel producing pairs, or short or long chains, never packets. Generally stain by Gram. Produce acid in dextrose and lactose broth. Pigment, if any, white or orange.

Subfamily Metacoccace e (Winslow and Rogers). Facultative parasites or saprophytes. Thrive best under aerobic conditions. Grow well on artificial media, producing abundant surface growths. Planes of fission often at right angles; cell aggregates in groups, packets, or.zoogloea masses. Generally decolorize by Gram. Pigment yellow or red.

The subfamily Paracoccaceæ must include, besides the two new color-types of the white and orange chromogens, the genera Diplococcus and Streptococcus, for which it was constituted in our earlier scheme. We have pointed out that the diplococci are characterized not only by their peculiar morphology but by strictly parasitic habit, very feeble growth on artificial media, and the possession of well-marked capsules under the proper conditions. Recent work, too, has shown that the fermentative reactions of the genus are characteristic. Comparative studies of the best known representatives of this group, the 
SUBFAMILIES AND GENERA OF COCCACEÆ 9I

pneumococcus and the meningococcus, have made it clear that they differ from the streptococci by their greater power of decomposing carbohydrates, - inulin, in particular, offering a fairly constant differential test. Furthermore, it appears that the diplococci are closely related in their response to immune sera, reacting to group agglutinins which do not affect the streptococci. In all respects, in habitat, in morphology, in growth on media, and in power of attacking carbohydrates, these organisms form the extreme end of the series of the Paracoccaceæ. The evidence on these points will be reviewed more fully in Chapter V.

It has seemed to us amply sufficient to warrant the recognition of the genus Diplococcus of Weichselbaum, with the following amplified definition:

Genus I. Diplococcus (Weichselbaum) Winslow and Rogers. Strict parasites, not growing, or growing very poorly, on artificial media. Cells normally in pairs, surrounded by a capsule. Fermentative powers high, most strains forming acid in dextrose, lactose, saccharose, and inulin. Hemolytic power generally lacking. Characteristic group serum reactions.

In one respect two species of diplococci depart from the characters of the subfamily as a whole. Both the gonococcus and the meningococcus decolorize by Gram. Such conditions as this are common in systematic biology, - some members of a group often differing radically in a single property from the general character- 
istics of the group as a whole. Classification, however, must be phylogenetic rather than logical; and there is no course but to include such aberrant forms in the group to which they are on the whole related.

The genus Ascococcus is a somewhat problematical one since so little work, and almost no comparative work, has been done upon the single peculiar type which it includes. The properties of this genus are more fully discussed in Chapter VI; but it seems most likely, in the light of present knowledge, that these slime-forming cocci of the sugar refineries are sufficiently peculiar to warrant generic rank. They are apparently more closely related to the diplococci and streptococci than to any other groups. Their cell grouping, in chains made up of paired units, their tendency to capsulation, and their high fermentative power, all ally them with these two genera. They may therefore be tentatively included under the Paracoccaceæ, in spite of their saprophytic habit. Until comparative study shall rectify or expand the characterization, we have defined the genus as follows:

Genus II. Ascococcus (Cohn) Winslow and Rogers. Saprophytic, growing vigorously in saccharine solutions. Cells in pairs, or in chains of paired elements. In presence of certain carbo-hydrates large, lobed gelatinous masses of zooglea formed. Fermentative powers high, acid being produced in dextrose, lactose, and saccharose.

The third genus of the Paracoccaceæ is the well-marked group of the streptococci. These organisms are commonly 
recognized by their characteristic morphology, meager growth on artificial culture media, and typically parasitic habit. Common serum reactions, too, show the close mutual relationships of the members of this group; but the conception of the genus is made much clearer by the comparison of the biochemical powers of these organisms, with those of other cocci. In our original series of 500, only I 7 organisms were included which formed the faint, veil-like surface growth of the streptococci. Even this small number of strains sufficed to show that these bacteria exhibit the generally positive Gram reaction of the Paracoccaceæ, but differ from the other members of the group in three points. They very rarely liquefy gelatin, and apparently never reduce nitrates, but exert an exceptionally powerful action on dextrose and lactose. We have since examined 44 more cultures of streptococci, making a total of $6 \mathrm{I}$ in all. The average acid production for the whole series was .oIg normal in dextrose broth and .oI4 normal in lactose broth. These figures are more than twice as high as those obtained for any other genus of the Coccaceæ in our investigation. It is important to notice, however, that these averages are composed in each case of two subordinate types within the genus. The actual distribution of the acidities in dextrose broth is plotted in diagrammatic form in Figure II, and it is there apparent that the curve for this genus has two distinct modes, one at an acidity between the neutral point and ..04, the other between .oro and .or 4 normal. It is the 
second subgroup which raises the average value for the genus. One of the most striking points about the curve is the

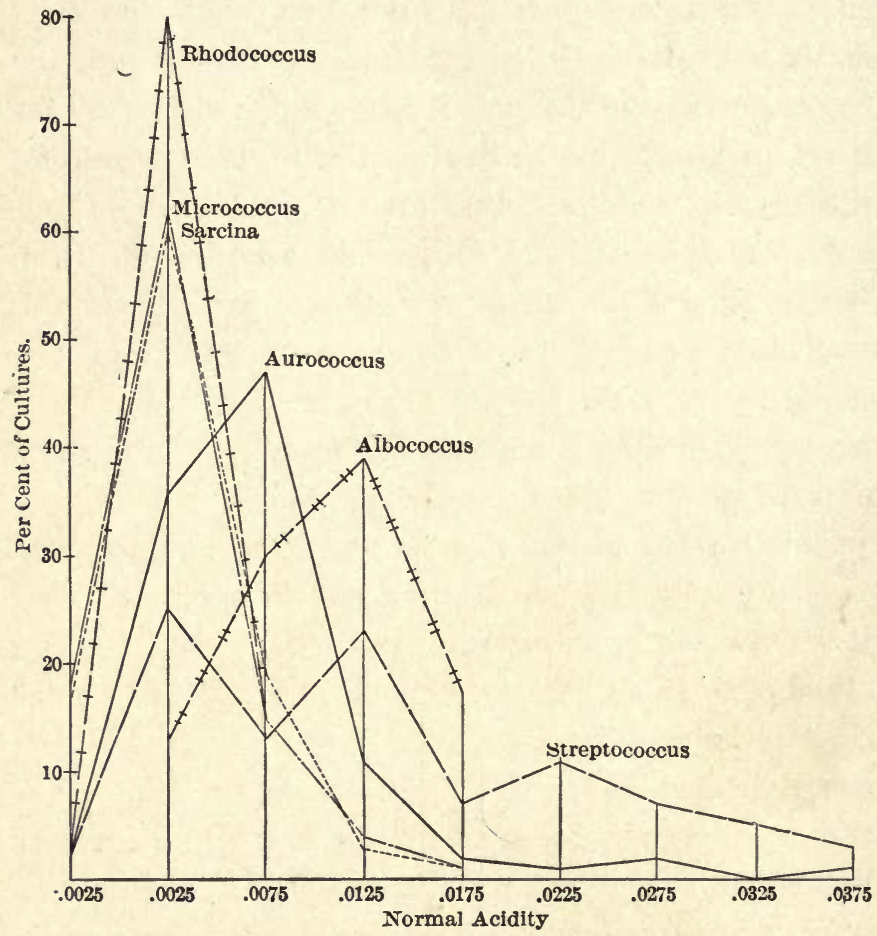

FIG. II. - Acid Production of certain Genera of the Coccaceæ in Dextrose Broth.

tendency exhibited to tail off very gradually toward extremely high acidities, - a phenomenon due, of course, to the presence of subgroups of very great fermentative power. 
In our series of cultures, there was a suggestive correlation between acid production and reaction to the Gram stain, the most actively fermenting forms being usually Gram-positive.

It is evident that the streptococci as a whole constitute an acid-forming, Gram-positive type; and these characters, with the general failure to attack gelatin and nitrates, may, therefore, be included in the characterization of the genus Streptococcus, in addition to its familiar morphological and cultural characters.

Genus III. Streptococcus (Billroth) Winslow and Rogers. Parasites. Cells normally in short or long chains (under unfavorable cultural conditions, sometimes in pairs and small groups, never in large packets). Generally stain by Gram. On agar streak, effused translucent growth, often with isolated colonies. In stab culture, little surface growth. Sugars fermented with formation of large amount of acid. Generally fail to liquefy gelatin or reduce nitrates.

There remain to be considered the four color-types of the White, Orange, Yellow, and Red cocci, the characters of which have been briefly discussed above. It will be remembered that the white and orange chromogens resemble the other Paracoccaceæ in failing to show the packet grouping, in a generally positive Gram reaction, in forming a surface growth of a not over-vigorous character, and in fermenting both dextrose and lactose. The yellow and red forms frequently show packets, are gen- 
erally Gram-negative, form good to abundant surface growth, produce only a slight acidity in dextrose and usually none in lactose. Minor characteristics are peculiar to each of the color-types.

On the whole the evidence seems amply sufficient to show that these four types constitute natural groups among the Coccaceæ. Each of them includes several distinct subtypes, characterized by such peculiarities as are ordinarily considered of specific value among bacteria. Each is marked by the correlation of other properties, of still greater systematic importance, which characterize the type as a whole. It seemed to us, therefore, that the four main types might well be given generic rank. It is a matter of more or less arbitrary opinion whether a given group of bacteria shall be considered a genus or not; but convenience certainly demands an increased use of generic names among these organisms. We have, therefore, separated the white and orange groups of the Coccaceæ from the genus Micrococcus with which they are generally included, under the new generic names Albococcus and Aurococcus; and have separated the red forms from the genera Micrococcus and Sarcina under the new generic name Rhodococcus (Winslow and Rogers, I906).

The characteristics of the genera into which the Coccaceæ are thus divided will be made clearer by the tabular statement on page 97 and by the diagrammatic representation of their respective average characters in Figure I. 


\begin{tabular}{|c|c|c|c|}
\hline \multicolumn{2}{|c|}{ Metacoccaceæ } & Paracoccaceæ & \\
\hline 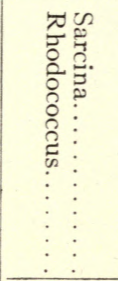 & 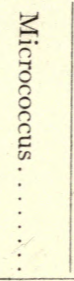 & 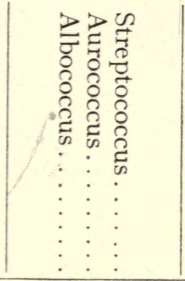 & ๑ొ \\
\hline$\stackrel{N}{+}$ & $\stackrel{N}{v}$ & ตู๐̆ & $\begin{array}{l}\text { Habitat (per cent } \\
\text { Parasitic Forms). }\end{array}$ \\
\hline $\overrightarrow{0}$ & ० & $0 \neq 0$ & $\begin{array}{l}\text { Cell-Grouping (per } \\
\text { cent of Packet- } \\
\text { Formers). }\end{array}$ \\
\hline$\infty \omega^{N}$ & N & +80 & $\begin{array}{l}\text { Gram-Stain (per cent } \\
\text { of }+ \text { Results). }\end{array}$ \\
\hline 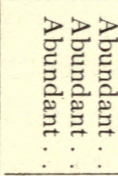 & $\begin{array}{l}0 \\
0 \\
0 \\
\vdots \\
\vdots \\
\vdots \\
\vdots \\
\vdots \\
\vdots\end{array}$ & 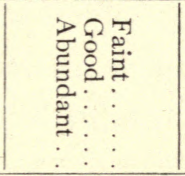 & 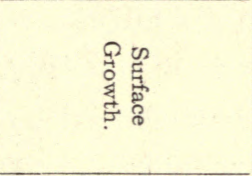 \\
\hline io & ¿ & ㅇํํ웜 & $\begin{array}{l}\text { Average Acidity in } \\
\text { Dextrose (Normal- } \\
\text { ity). }\end{array}$ \\
\hline$\dot{8}$ & $\dot{\circ}$ & 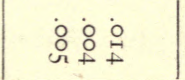 & $\begin{array}{l}\text { Average Acidity in } \\
\text { Lactose (Normality) }\end{array}$ \\
\hline ज̆ & $\stackrel{N}{N}$ & $\stackrel{\omega}{H} O$ & $\begin{array}{l}\text { Nitrate Reduction } \\
\text { per cent of Reducers. }\end{array}$ \\
\hline $\begin{array}{l}\pi \\
0 \\
2 \\
\vdots \\
\vdots \\
\vdots \\
\vdots \\
\vdots\end{array}$ & $\begin{array}{l}\stackrel{1}{0} \\
\vdots \\
\vdots \\
\vdots\end{array}$ & 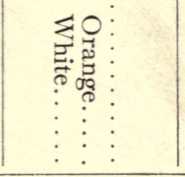 & 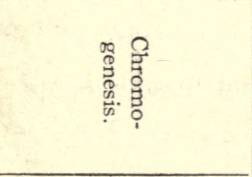 \\
\hline$\infty$ 워 & ळ & Я્ન & $\begin{array}{l}\text { Gelatin (per cent of } \\
\text { Liquefiers). }\end{array}$ \\
\hline$\dot{i}$ in & in & $\begin{array}{l}-1 \text { in: } \\
H \text { n: }\end{array}$ & $\begin{array}{l}\text { Liquefaction (Aver- } \\
\text { age Liquefaction, } \\
\text { cm.). }\end{array}$ \\
\hline
\end{tabular}


Certain characters are included in the table which have been omitted from Figure I and vice versa; but the two together furnish a clear picture of the different groups. The contrast between the Paracoccaceæ and the Metacoccaceæ in regard to habitat, 'gram stain, surface growth, and acid production is brought out in Figure I. The table shows in addition the difference in average acid production. The peculiar relations of temperature to chromogenesis in Aurococcus and Rhodococcus are shown in the figure, as well as the high liquefying power of Aurococcus. The slight action of Rhodococcus upon gelatin, and the high reducing power of this genus, are indicated in the table.

The generic name Aurococcus was suggested by us for the cocci characterized by the production of an orangeyellow pigment, corresponding to the eighth and ninth chromas under the Cadmium Orange column of the frontispiece. These forms have previously been grouped with the yellow chromogens in the genus Micrococcus. Their distribution is, however, about a center quite distinct from that of the yellow micrococci, as indicated in the frontispiece; and in all their characters the orange and yellow forms are strikingly different.

Of our 500 cultures 180 fall naturally in the genus Aurococcus. Of these, I35, or 76 per cent, were isolated from the human body. Eight strains only, or 4 per cent, showed packets. On the other hand, 124, or 69 per cent, showed a uniformly positive reaction to the Gram stain, 
while only Io, or 6 per cent, were Gram-negative on both trials. In both sugar broths the activity of these forms was considerable, tho less than that of the streptococci. In dextrose the average acidity was .007 normal; in lactose .004 normal. The actual distribution of the cultures in regard to dextrose fermentation is indicated in Figure II. The mode is there seen to come between .005 and .009 normal; and it is interesting to note the same tendency to fall off very gradually toward the higher acidities, which was noticed among the streptococci. Forty strains, or 22 per cent, of the orange cocci grew distinctly better at 37 degrees than at 20 degrees, a higher figure than appeared in other genera. In regard to chromogenic power, however, a very characteristic relation was apparent. One hundred and twenty-six cultures, or 70 per cent, showed better color production at 20 degrees than at 37 degrees. In regard to gelatin liquefaction, it appeared that 54 cultures, or 30 per cent, failed to exert any action in the 30 days' time for which this property was observed. The extent of the effect produced by the cultures which did liquefy was very notable.

On the average the depth of liquefaction produced after 30 days was 2.2 centimeters, nearly double the value observed for any other genus; and the curve of distribution for this character in Figure III makes it clear that the aurococci which do liquefy exert a much more vigorous action than is characteristic of any of the other genera. 
The genus Aurococcus, therefore, presents a fairly definite picture; and on the strength of these observations we have defined it as follows:

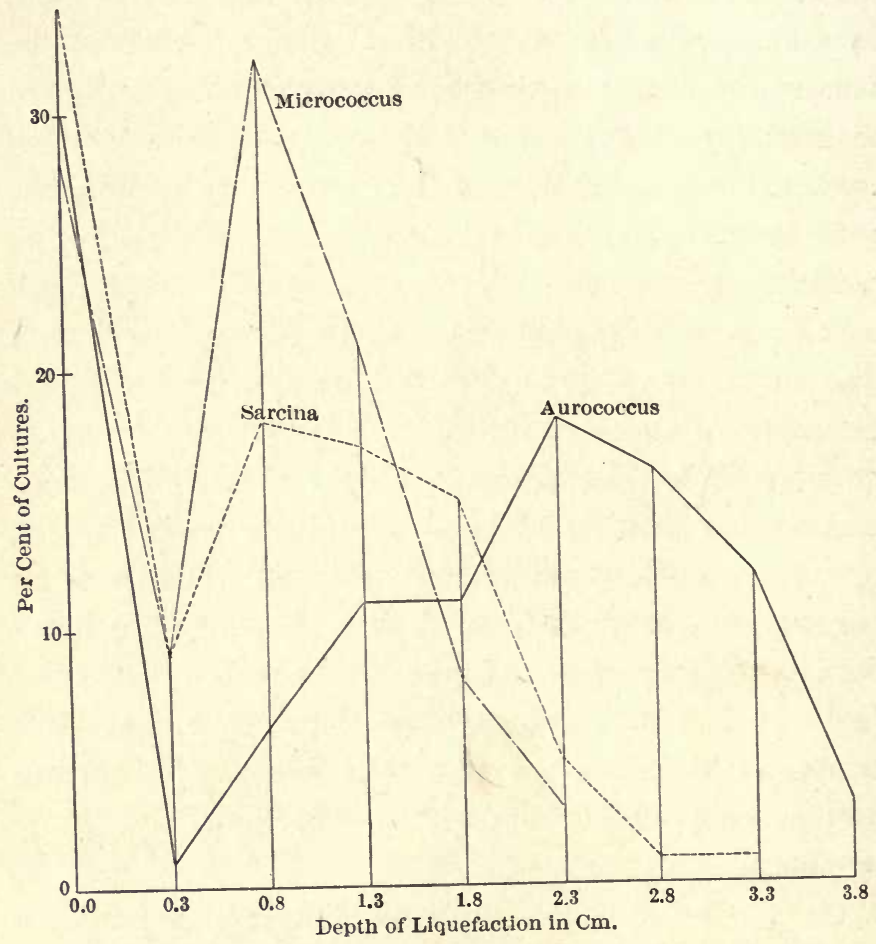

Fig. III. - Liquefaction of Gelatin by certain Genera of the Coccaceæ.

Genus IV. Aurococcus (Winslow and Rogers). Parasites. Cells in groups and short chains, very rarely in packets. Generally stain by Gram. On agar streak 
good growth of orange color. Sugars fermented with formation of moderate amouni of acid. Gelatin often liquefied very actively. May or may not reduce nitrates.

The white staphylococci, for which we created the genus Albococcus, like the aurococci, showed positive Gram reactions and were generally of parasitic habit, altho a considerable proportion ( 7 out of 23 cultures) were isolated from the air. They differed again from the saprophytic micrococci, with which they have been included by Migula and Chester, by their high fermentative power. It will be noticed that in Figure II their acid production appears higher than that of Aurococcus, having its mode between .oro and .or4 normal. All these properties - habitat, Gram reaction, acid producing power - clearly ally these white forms with the Paracoccaceæ. On the other hand, in color as well as in amount of growth mass, they differ from the aurococci. Their surface growth on agar is distinctly heavier than that of the orange forms, and porcelain white or grayish white, in color, with no tinge of orange. Furthermore, among the white cocci the rapid action upon gelatin, noted among the aurococci, was apparently lacking. The average depth of liquefaction among the I2 cultures, which liquefied at all, was only I.I centimeters, corresponding to that observed among the yellow saprophytes.

The number of cultures with which we worked was small; but the creation of a new genus, in this case, is justified by a consideration of the independent evidence of 
other observers. Gordon (I905) made a careful study of the white staphylococci which will be discussed more fully in Chapter IX. He examined 300 different strains in eight differential media; and his results clearly indicate the existence of a natural group of cocci which corresponds to the one suggested by our small series of cultures. All of his 300 white skin cocci were Gram-positive. The commonest type coagulated milk, liquefied gelatin, reduced nitrates, and showed high fermentative powers. Gordon's work, however, threw no direct light on the relation of these organisms to the orange forms. Dudgeon (I908), in a more recent paper, reports a comparative study of the orange and white staphylococci, inspired by the work of Andrewes and Horder. He observed the liquefying power, the reducing power (as shown by lead acetate and neutral red), and the acid production in eleven carbohydrate media, of 46 strains of the orange type and $7 \mathrm{I}$ of the white. He concluded that all the staphylococci studied were members of one species, altho only a few of the strains were identical in all reactions. None of his tests were quantitative, and no attempt was made to analyze the figures with regard to the numerical distribution of various types. An examination of Dudgeon's own tables shows that marked differences existed between his two groups of organisms. All but two of his orange strains liquefied gelatin, while only 60 per cent of his white strains did so. Neutral red was reduced by 60 per cent of the orange strains and by only 5 per cent of the 
white ones. Eighty per cent of the orange forms acidified mannite, against 50 per cent of the whites. In glycerin, 70 per cent of the orange strains and 55 per cent of the white ones were positive. Raffinose was attacked by twothirds of the orange forms and by only 40 per cent of the white ones. In the absence of quantitative data it is difficult to know how much this means, but it indicates a distinct difference between the groups.

It is clear that as actually found, in the body or on its surface, some cocci produce white and some, orange growths. Dudgeon acknowledges the force of this fact (altho he is misled as to the importance of chromogenesis by the irrelevant circumstance that colonies of orange cocci in a crowded plate may remain white). He points out too that the white cocci are uniformly associated with less serious pathological lesions than the orange forms. These two facts are universally obvious to all students of suppurative processes. When, in addition, it appears that even a small series of the white forms show distinct group differences from the aurococci in relation to dextrose and lactose and gelatin, the recognition of a generic group seems warranted.

We have defined the genus Albococcus by its more salient and obvious characters alone. Dudgeon's paper suggests that the reducing power of these organisms, and their action upon mannite, glycerin, and raffinose, may also prove significant; but quantitative studies must be carried out before this is established. 
Genus V. Albococcus (Winslow and Rogers). Parasites. Cells in groups and short chains (never in packets). Generally stain by Gram. Growth on agar streak abundant, and porcelain-white in color. Sugars fermented with production of a moderate amount of acid. Gelatin liquefaction and nitrate reduction may or may not occur.

This genus closes the series of the Paracoccaceæ. The saprophytic forms, the Metacoccaceæ, are distinguished from all the types so far defined by their generally negative Gram reaction and their low fermentative power. They are grouped about two color-types, the red and the yellow, which are characteristically saprophytic rather than parasitic in habit; and one of these, the red type, has special peculiarities of its own.

The first group of the Metacoccaceæ includes the yellow cocci. These organisms frequently show the packet grouping. Two hundred and fifty-one, or over half of our 500 cocci, fell in this group and packet formation was observed in $\mathrm{r} 37$ cultures out of the $25 \mathrm{I}$. The yellow type includes a large number of the forms described as species in the genera Micrococcus and Sarcina. In our series the properties of the packet-formers and the non-packet-formers ran almost exactly parallel; and it is doubtful whether the single property of the sarcina grouping is of sufficient importance to warrant generic rank. The names Micrococcus and Sarcina are, however, so firmly established in the literature of bacteriology that we have hesitated to disturb them. 
The chromogenic type center for both micrococci and sarcinæ lies in the lighter shades of the Medium Cadmium Yellow (see frontispiece). Both are typically saprophytic in habit; 73 per cent of our micrococci and 76 per cent of our sarcinæ were from water, earth, or air. They are generally Gram-negative; only 22 and 23 per cent, respectively, gave two successive, positive results. In fermentative power micrococci and sarcinæ correspond closely, as indicated in Figure II. In each case a very faintly acid or neutral reaction was the general rule. A few strains, about 20 per cent in each case, formed an acidity over .004 normal in dextrose broth, and about Io per cent exceeded a similar limit in lactose broth. In relation to temperature, and in chromogenic power also, micrococci and sarcinæ agree. In all these respects the parallelism of these two series of the yellow cocci emphasizes the differences which separate them both from the Paracoccaceæ.

Two characters only appear, from a careful comparison, to distinguish the packet-forming and non-packet-forming yellow cocci. The sarcinæ produce, on the whole, more vigorous surface growths than the micrococci. Thirtythree per cent of the former produced a growth designated as "very heavy" in our observations; while only 8 per cent of the micrococci showed a growth of this kind. And in gelatin cultures the sarcinæ exert a more vigorous action than the micrococci, as appears in Figure III. A difference of the latter sort may be due to the pro- 
duction of more active enzymes of a particular type; or it may simply be associated with greater vigor of growth and multiplication. It is possible that these two characters, which differ in the sarcinæ and micrococci, may both be expressions of a greater general vigor, which manifests itself in the property of packet formation as well. If such be the case, the generic difference between these types can scarcely be maintained. Without absolutely conclusive evidence of identity, it does not, however, seem wise to overrule genera which have become so firmly established by long usage. We have, therefore, included in our scheme of the Coccaceæ two yellow types, Micrococcus and Sarcina, distinguished from each other by the presence or absence of packet formation, but alike in all other respects. These genera are defined as follows:

\section{Genus VI. Micrococcus (Hallier) Winslow and} Rogers. Facultative parasites or saprophytes. Cells in plates or irregular masses (never in long chains or packets). Generally decolorize by Gram. Growth on agar abundant, with formation of yellow pigment. Dextrose broth slightly acid, lactose broth generally neutral. Gelatin frequently liquefied. Nitrates may or may not be reduced.

Genus VII. SARcina (Goodsir) Winslow and Rogers. Facultative parasites or saprophytes. Division occurs under favorable conditions in three planes, producing regular packets. Generally decolorize by Gram. Growth on agar abundant, with formation of yellow pigment. Dextrose broth slightly acid, lactose broth generally neutral. 
Gelatin frequently liquefied. Nitrates may or may not be reduced.

The last group - which includes the red chromogenic cocci - is so individual in its characteristics that even our small series of cultures was sufficient to establish it as a clearly defined type. Twenty-five of our 500 cocci formed a reddish or pinkish growth. The shade of color produced varied considerably, and these organisms were scattered rather irregularly over the last three columns of the chart of color distribution (see frontispiece); but the group proved to be an exceptionally homogeneous one. Only one culture out of the 25 came from a parasitic habitat; and only 2 of the 25 showed two successive positive Gram reactions. In fermentative power the red cocci corresponded closely with the yellow types. It will be noticed in Figure II that, like the micrococci and sarcinæ, they center about a low acidity; and there is even less variation among the red than among the yellow forms. The growth masses of the red cocci were on the whole more luxuriant than those of any other type. Twentythree of the 25 strains were classed as abundant in growth.

In these aspects the red cocci correspond with the other types of the Metacoccaceæ. They form apparently the extreme end of a series which begins with some of the less vigorous micrococci. Among the red forms the saprophytic habit, the negative Gram stain, and the vigor of surface growth all reach a maximum. In chromogenic 
power and in relation to gelatin and nitrates the red cocci exhibit characters which are peculiar to themselves. Reference to the comparative table on page 97 shows that in the white, orange, and yellow groups between 60 and 70 per cent of the cultures studied liquefied gelatin; and the average depth of liquefaction after 30 days was from I to 2 centimeters. Among the red cocci, on the other hand, only 2 out of 25 strains affected the gelatin in this time and in each case to a depth of less than half a centimeter. An examination of the published descriptions of red cocci in the literature shows that these organisms are in general characterized by extremely sluggish and uncertain peptonizing power. Their action upon nitrates is equally peculiar. From to to 30 per cent of the organisms of other color-types attacked nitrates, and, of the reducers, half formed ammonia and half nitrites. Of the red cocci, on the other hand, I 4 out of 25 , or $5^{6}$ per cent, reduced nitrates; in every case nitrites were formed but not ammonia.

Evidently the red cocci constitute a natural group of considerable definiteness; and for this group we have suggested the generic name Rhodococcus. Molisch (I907) has since applied the same name to a genus of the purple bacteria; but our use of the name has priority by about a year. Both packet-forming and non-packetforming types are included in the genus; Io of the former and $I_{5}$ of the latter occurred in our series. In no other respect were the two types distinguished. Both showed 
SUBFAMILIES AND GENERA OF COCCACEÆ IO9

the same color, the same general cultural and biochemical properties, the same peculiar relation to gelatin and nitrates. There is no established distinction to be deferred to here as in the case of Micrococcus and Sarcina; and there is clearly no ground for creating a new distinction between forms so closely allied as all the red cocci appear to be. We have, therefore, defined the genus Rhodococcus so as to include all forms which exhibit the peculiar complex of characters which has been described above, whether their cells occur in packets or in smaller and less regular groups.

Genus VIII. Rhodococcus (Winslow and Rogers). Saprophytes. Cells in groups or regular packets. Generally decolorize by Gram. Growth on agar abundant, with formation of red pigment. Dextrose broth slightly acid, lactose broth neutral. Gelatin rarely liquefied. Nitrates generally reduced to nitrites, but not to ammonia. 


\section{CHAPTER V.}

\section{THE GENUS DIPLOCOCCUS.}

THE genus Diplococcus of Weichselbaum has been defined above as follows:

Strict parasites, not growing, or growing very poorly, on artificial media. Cells normally in pairs, surrounded by a capsule. Fermentative powers high, most strains forming acid in dextrose, lactose, saccharose, and inulin. Hemolytic power generally lacking. Characteristic group serum reactions.

The type organism of this genus is $D$. pneumonice (Weichselbaum); this species and its allies, the gonococcus and the meningococcus, all occur in capsulated pairs, as the causative agents in the specific diseases with which they are associated. None of the three are known to occur outside the animal body.

These diplococci have been grouped by most systematic bacteriologists (Migula, I900; Chester, I90I) with the streptococci. It seemed to us, in our first revision of the Coccaceæ (Winslow and Rogers, I905), that the typical growth form and strict parasitic habit warranted the retention of the distinct genus Diplococcus for the three types mentioned. Since that time evidence has accumulated, from the study of biochemical properties 
and serum reactions, which establishes the individuality of the genus beyond reasonable doubt.

The first important biochemical test which distinguishes the diplococci from the streptococci is their difference in fermentative powers. This property was at first studied in a curiously roundabout way. Libman (I900 and I90r) noted that when bacteria are grown on media containing serum and sugars there may occur a white opacity connected with the precipitation of some constituent of the serum; and showed that this action was due to acid production. Hiss (1902), in a fruitful series of investigations, found that both pneumococci and streptococci uniformly ferment the monosaccharides, dextrose, levulose, and galactose. The pneumococci always ferment the disaccharides, lactose, saccharose, and maltose, and the streptococci generally do so, altho some cultures fail. The pneumococci produce acid in serum media containing dextrin, starch, glycogen, or inulin, as manifested by the resulting coagulation of the serum proteids; while the streptococci generally fail to do so with the first three polysaccharides and always fail with inulin. Finally, the pneumococci produce, in a diluted alkalin ox-serum, to which no sugar has been added, sufficient acid to cause coagulation. Berry (1907) has recently shown that the inulin reaction of the pneumococcus, like other characteristics, is subject to considerable variations. Cultivation of this organism on artificial media causes modifications of the diplococcus morphology toward the 
streptococcus type, lowered virulence, and the loss of the inulin-fermenting power. This variability under artificial conditions does not, however, alter the fact that the properties mentioned are characteristic of the pneumococcus as actually found in the body.

Another important difference between streptococci and diplococci, observed first by Schottmüller (1903), lies in the fact that the former frequently exhibit hemolytic power, while the latter do not. The most typical strains of Str. pyogenes, when grown upon blood agar, form grayish colonies surrounded by a clear zone from which the hemoglobin has been entirely removed. This type Schottmüller called Str. pyogenes seu erysipelatos. On the other hand, D. pneumonia, the zooglœa-forming diplococcus and certain streptococci (called by Schottmüller Str. mitior seu viridans), form in blood agar greenish colonies, without obvious solution of the hemoglobin. In blood-broth, Str. pyogenes produces a carmine red coloration, while organisms of the second type change the solution to a brownish hue. Rosenow (I904) independently observed this characteristic action of the pneumococci; and explained the brownish turbidity formed in blood-broth as due to the action of the organism upon the hemoglobin.

That both the Hiss reaction and the Schottmüller reaction are complex phenomena is indicated by some recent work of Longcope's (I905). He finds that blood sera may or may not contain in themselves substances 
from which $D$. pneumoniae produces acid, and that the coagulation in presence of acid is markedly influenced by heating the serum. Furthermore, it occurs, under certain conditions, only at a certain optimum acidity, failing when the serum is heated in the presence of a larger amount of acid.

The most important work upon this group of cocci, and one of the best-planned campaigns ever executed in bacteriological research, was the investigation carried out by a number of independent observers under the auspices of the Medical Commission for the Investigation of Acute Respiratory Diseases, appointed on the initiation of the Health Department of the City of New York. That part of the work which bears upon the systematic relationship of the diplococci and their allied forms may be briefly summarized as follows:

Park and Williams (I905), the first of the collaborators, studied one hundred and forty strains of pneumococci. They distinguished as typical those which occurred in elongated pairs, showed capsules, coagulated inulinserum media, and produced a green coloration on bloodagar plates. Among these, three variants were noticed, one with small cells and narrow capsules, one with large cells and broad capsules, and a zooglœa form, with cells in chains of pairs. The latter is commonly known as D. mucosus in America; but, as shown later, it should rather bear the name $D$. involutus, Kurth. In addition, two "atypical" forms were found, one resembling the 
pneumococcus in morphology but failing to form acid from inulin, the other resembling Str. pyogenes in form, but coagulating the inulin-serum. Both morphology and inulin-fermenting power varied somewhat, even in the "typical" forms. All the cultures tested fermented dextrose, lactose, and saccharose, but about a third of the strains failed to form acid from mannite.

Longcope and Fox (I905) compared the characteristics of organisms from normal saliva with those obtained from infections supposed to be pneumococcic and streptococcic respectively. Sixteen of the strains from pneumococcic infections were typical $D$. pneumonia; one, however, failed to coagulate the inulin-serum medium. Of eleven strains from streptococcic infections, none showed capsules, none fermented inulin, and most showed hemolytic action. On agar the colonies were dry and granular, while those of the pneumococci were raised and moist. Of the cultures from the normal throat, nineteen resembled pneumococci, fermenting inulin, tho somewhat slowly, and showing a low virulence. The other sixteen saliva cultures resembled the streptococci in all the characters noted above, tho generally appearing in pairs of lanceolate cells. All the cultures studied stained by Gram. The authors failed to find any constant types of pathological lesion produced by different strains of pneumococci such as Eyre, Leathem, and Washbourn (1906) have described.

Norris and Pappenheimer (I905), in a study of organisms isolated from the mouth and lungs after death, 
divided them into five groups as follows: (I) typical pneumococci (including forms which produce acid in inulin but in insufficient amount to coagulate serum); (2) Str. mucosus (distinguished from the first class by abundant moist growth and chain formation); (3) inulin-fermenting diplococci without capsules; (4) streptococci, with no capsule, but fermenting inulin; (5) typical streptococci (no capsule, no fermentation of inulin). The relative proportion of the various types is significant; typical pneumococci were isolated twenty-one times and typical streptococci twenty-four times, while only three cultures fell in group 2, seven in group 3, and eight in group 4.

Duval and Lewis (1905), working in Boston, studied the inulin reaction with special detail, and found marked variations among organisms which they considered as undoubted pneumococci. They distinguished high acid producers (also fermenting mannite), medium acid producers, low acid producers, and forms which failed entirely to ferment inulin altho showing the typical morphology.

Buerger's (1905) results were essentially similar to those of Park and Williams. Of some fifty cultures of pneumococci, most showed the typical morphology, all fermented lactose and inulin, and most were virulent. A few showed cells smaller than the average, a few, rounded cells, and a few, cells more elongated and rod-like than the normal. Two cultures exhibited the wide capsule and large slimy colonies of Str. mucosus; and several minor 
varieties were described. The organisms found in the throats of healthy persons could not be distinguished from those in the throats of pneumonia cases. Agglutination reactions were not sufficiently specific to be of any assistance in separating the varying types.

Finally Hiss (1905), at the central laboratory of the commission, made a comparative study of two hundred and sixty distinct organisms, obtained largely from the individual observers previously cited. He distinguished eight different types, according to variations in morphology, acid production, and agglutination reactions, four showing the diplococcus morphology, fermenting inulin and reacting with pneumococcus sera, and the other four showing the streptococcus morphology, failing to act on inulin, and not agglutinated by pneumococcus serum. Differences in the shape of the cells, in the capsule and in the action on blood agar distinguished minor groups; but the only sharply marked variety was the Str. mucosus, differentiated from $D$. pneumonice by chain formation and by its large, smooth, shiny colonies. Other variants differed from the pneumococcus by the absence of capsules alone, and others again differed from the streptococcus only by fermenting inulin. The Schottmüller reaction appeared less specific for the pneumococci than the action upon inulin.

Variability is evidently great among the pneumococci, as elsewhere in the Paracoccaceæ. Kruse and Pansini (I892), not long after the first discovery of the organism, 
examined in detail eighty-four different cultures of forms allied to the pneumococcus, isolated from sputum and from various pathological conditions, and observed marked variations but no clearly defined varieties. "We find," they conclude, "a certain sum of common characters which taken together give a picture of the Diplococcus lanceolatus capsulatus but we find also numerous differences, quantitative and qualitative, in virulence, in growth characters and in viability." Morphologically, all gradations appeared between cultures which showed chains of spherical cells with faint capsules and those which showed, almost exclusively, typical pairs of encapsulated lanceolate cells. Continued cultivation on artificial media tended to develop more and more clearly the first type of growth, while animal inoculation often restored the second. Virulence could be diminished or increased by proper treatment. Those forms which most resembled Str. pyogenes in morphology showed also a more vigorous growth on artificial media, and at low temperatures. Coagulation of milk varied notably in different strains, and in the same strain at different times.

Kindborg (1905) has recently emphasized again the existence of numerous slightly differing varieties among organisms supposed to be pneumococci. He studied twenty-four races from sputum and pus, and among these, some were constantly larger or smaller than the type, and others showed a definite tendency to chain formation. Variations in vigor of growth and in temperature 
relations were marked, the less virulent organisms being best adapted to artificial media. Several cultures coagulated milk and one liquefied gelatin. The pathogenicity varied markedly, the cultures from pneumonic sputum being most virulent and those from pus generally nonvirulent. Agglutination reactions varied with the race.

Eyre, Leathem, and Washbourn (1906) claim that two distinct types of pneumococci may be distinguished by the local lesions they produce in the tissue of the rabbit. One form arouses a fibrous, and the other a cellular, exudate in the subcutaneum. Among the fourteen organisms studied by them were three which failed to ferment mannite and four which failed to ferment inulin. The authors were able to transform pneumococci of high virulence and poor vitality on media into forms of low virulence and vigorous saprophytic growth, by artificial cultivation, and to restore their original characters by animal inoculation.

On the whole this mass of investigation has made it clear that the conception of numerous varieties, clustered about certain well-marked centers, exactly fits the case of the cocci of the nose and throat. Two such type centers stand out with remarkable clearness and justify the retention of the two distinct genera, Diplococcus and Streptococcus. The first, exemplified in $D$. pneumonice, is a lance-shaped coccus, occurring in pairs and surrounded by a capsule. It grows feebly on ordinary culture media, but exhibits active fermentative powers, splitting mono- 
saccharides, polysaccharides, and inulin. It produces acid and a greenish coloration in serum-media, but does not exert hemolytic action; and it reacts to more or less specific group agglutinins. The Str. pyogenes type differs in all of these respects. Between the two extremes, organisms are found which exhibit almost every grade of intermediate character, in morphology, cultural features, fermentative power, and serum reactions. These are evidently transitional forms between the commoner and more stable types; and it seems hardly wise to give definite names to the variety of $D$. pneumonice without capsules or the Str. pyogenes that ferments inulin.

The type species of the genus Diplococcus is clearly D. pneumonice (Weichselbaum). This organism was first described by Friedlander (I883), and named a few years later by Weichselbaum (I886). Synonyms are B. salivarius-septicus, D. lanceolatus-capsulatus-pneumonicus and Str. lanceolatus. It occurs in the exudate and in the lungs in many cases of pneumonia and is probably the most frequent cause of that disease. The organism is round or elliptical in shape, often with pointed or lanceolate ends; it occurs most commonly in pairs, and in the body is generally surrounded by a capsule.

On artificial media chains are frequently observed. The organism is typically Gram-positive. In cultures D. pneumonia grows at temperatures over 25 degrees, tho not vigorously, and develops best away from the air. Heyrovsky (1905) found that the rapid death of 
D. pneumonice in certain artificial media is due to the acid reaction which it produces, and may be forestalled by carefully repeated neutralization of the culture medium. The same author cites the interesting fact, noticed by several observers, that the diplococcus forms long chains when grown in serum containing its anti-bodies, just as it does when grown in artificial media.

The growth of the pneumococcus in the stab, or on agar plates, closely resembles that of Str. pyogenes but is in general less vigorous. Its colonies are thin and translucent. Buerger (1905) notes that on glucose-ascites-agar the colonies, when seen from the side, show a series of milky rings inclosing a transparent center. On blood agar greenish colonies are produced with no hemolysis. As noted in the previous discussion of the genus as a whole, the fermentative powers of $D$. pneumonia are high, acid being typically formed from dextrin, starch, glycogen, and inulin, and even in diluted alkalin ox-serum without the addition of any foreign carbohydrate.

The virulence of the pneumococcus, like that of other cocci, varies materially. A culture of exalted virulence, injected into susceptible animals, mice or rabbits, produces death from general septicæmia. In more immune animals a local inflammation follows, which may resemble pneumonia if the lung tissue is the one affected

On the whole there does not seem to be conclusive evidence for the existence of more than one type center among the pneumococci. Individual varieties exist in 
great numbers; but they all appear to group themselves about a single type, and this type may be defined as follows:

I. D. Pneumonia (Weichselbaum). A coccus occurring commonly in the body, in pairs of elongated lanceolate cells, surrounded by a capsule. Stains by Gram. On media often in short chains. Growth on media faint, producing small translucent disk-like surface colonies. Ferments monosaccharides, disaccharides, and inulin. Lacks hemolytic power. Virulence very variable. Shows characteristic group serum reactions. Occurs in normal or pneumonic sputum and in infected tissues in pneumonia.

Whether pneumonic infections of horses and cattle are due to specific organisms allied to $D$. pneumonice is not yet certain. The descriptions of the various forms isolated from such sources are so imperfect that it is even uncertain whether they are cocci or bacilli.

Two other pathogenic diplococci, D. Weichselbaumii and $D$. gonorrhoee, clearly belong to this genus; and they and their varietal strains form a well-marked series, distinguished from $D$. pneumonice and its allies by morphological and cultural relations. Both organisms occur in pairs of flattened cells instead of lance-shaped ones. Both are Gram-negative, and both grow much less readily on artificial media than does $D$. pneumonia. D. gonorrhoee (Neisser, Flügge), was discovered by Neisser (1879) and was known as the Gonococcus until given a specific name by Flügge (I886). It was first 
described on the basis of its morphological characters alone, as a coccus occurring in pairs of hemispherical, "coffee-bean" cells, found in greatest numbers inside the pus cells of the diseased secretion which it excites. It stains easily and decolorizes easily both by the Gram and Neisser methods. The organism is strongly parasitic and may be grown on artificial media only with difficulty. Bumm (I885) first succeeded in cultivating it on serum. It has since been claimed by various observers that $D$. gonorrhere will grow on ordinary media if the reaction be made slightly alkalin (Vannod, I905). Colonies of the organism appear as small grayish-white points like those of $D$. pneumonice, but of somewhat denser and more viscid consistency. Little or nothing is known of its biochemical powers.

D. gonorrhoee has never been found outside the human body. It occurs typically, as pointed out above, in the pus cells in cases of gonorrheal disease and is occasionally associated with secondary arthritis in affected persons. Its virulence for experimental animals is insignificant. Inoculation produces a more or less severe temporary inflammation, but the gonococci rapidly die out and disappear.

In this type again we find a number of related but slightly differing strains. Bumm (1884) showed long ago that forms closely resembling $D$. gonorrhoee in morphology may be found on the conjunctiva without the occurrence of gonococcal infection, and that occasionally 
strains appear which can scarcely be distinguished in cultural relations, or in any other character than pathogenesis, from the gonococcus.

Schanz (Ig04) has recently summarized evidemce from many other sources as to the existence of atypical forms, of the gonococcus or of forms allied to it, but differing in growth on media, and in reaction to the Gram stain.

The central type form may be defined as follows:

2. D. GONORRHEE (Neisser, Flügge). $A$ coccus occurring commonly in pairs of flattened coffee-bean shaped cells. Decolorizes by Gram. Fails to develop, or develops very feebly, on ordinary media. Not pathogenic for animals. Occurs most abundantly in pus cells in gonorrheal infections. Exhibits group serum reactions with $D$. Weichselbaumii.

$D$. Weichselbaumii, a form closely related to $D$. gonorrhœe from the bacteriological standpoint, was first clearly distinguished by Weichselbaum (1887), as the commonest causative agent in epidemic cerebro-spinal meningitis; and was named by him D. intracellularis meningitidis. The trinomial specific name was replaced by Trevisan (I889) with a proper binomial, D. Weichselbaumii.

This coccus is found in the cerebro-spinal exudate,in largest numbers inside the leucocytes and other body cells. Its characters have been very fully studied by Bettencourt and França (I904). They found it occurring usually in pairs of flattened cells, often in fours and irregular masses, never in chains. It decolorized by 
Gram. Scum and turbidity appeared in broth (containing cerebro-spinal fluid), and on ascites-agar the organism appeared in small yellowish brown colonies with crystalline deposits at their margin. The behavior of the coccus in other media, and under the action of various unfavorable agents, is fully described in the original paper. In general, the meningococcus differs from the gonococcus, culturally, by the fact that it grows more readily on ordinary media. In this respect $D$. Weichselbaumii is intermediate between $D$. pneumonice and $D$. gonorrhoee.

The meningococcus exhibits a slight degree of virulence for the ordinary laboratory animals, being more pathogenic than $D$. gonorrhoee but much less pathogenic than the pneumococcus.

Dr. Wollstein (1907) and other observers have recently shown that $D$. gonorrhoce and $D$. Weichselbaumii are closely related in their reactions to immune sera. Agglutinins, aggressins, protective power, and amboceptors developed in the bodies of immunized animals are common to the two forms and distinct from the products characteristic of Str. pyogenes and D. catarrhalis. The better viability of $D$. Weichselbaumii on culture media and its somewhat higher virulence are the only distinguishing marks of the two species, aside from their differing pathogenic effects in man.

As in the other types of this genus, a long contest has been waged over the problem of variability among the meningococci. Jaeger (1902) and others claimed to 
have found races of the meningococcus which showed short chains, at times, which stained by Gram, and grew at 20 degrees; while Weichselbaum (1902), and his associates at Vienna (Albrecht and Ghon, I902), stoutly maintained that the true $D$. Weichselbaumii never formed chains of more than four elements, never stained by Gram, and never grew at 20 degrees.

Jaeger (I9O3) reported that meningococci of both types exhibited specific agglutination reactions in immunized rabbit's blood, while slightly different organisms, and those derived from other sources than meningitis, were differently affected. D. catarrhalis was agglutinated by physiological salt solution!

Libman (I902) isolated only the typical form of D. Weichselbaumii from the New York cases studied by him, and was inclined to discredit the findings of the Jaeger school. The evidence for the existence of atypical meningococci is, however, too considerable to be lightly put aside. Lepierre (I904) studied D. Weichselbaumii with considerable care and came to the conclusion that this species, like others, exhibits widely varying races. As obtained from the spinal fluid, the organism appeared only in characteristic pairs of flattened cells; but on cultivation in ascites-bouillon, chains appeared more and more frequently in successive generations. The ability to grow on artificial media rapidly increased during the same process. After this development of vigor by artificial cultivation the organisms were capable, in large 
doses, of killing rabbits, and a few passages through the animal body produced a considerable degree of virulence. With increasing virulence the relation to the Gram stain also changed. Forms of low virulence generally decolorized; but eight or ten passages through rabbits produced a race which was uniformly Gram-positive. The type with which Lepierre started, in each of three separate cases, was the Weichselbaum type of the meningococcus, with no chains, negative Gram reaction, feeble growth on ascitesmedia, and low virulence. The final result of successive cultivations and animal inoculations was the JaegerHeubner type, which stands at the opposite extreme in all these respects. Sorgente (1905), too, has shown that the two types of the meningococcus interchange their characters under varying conditions of cultivation, and that they are not distinguished by specific serum reactions.

It seems on the whole certain that races of the meningococcus may occur which vary from type in viability, staining reactions, and morphology. According to the view of bacterial species which we have been led to adopt this would naturally be the case. The common central type under normal conditions, however, is the one described by Weichselbaum; and Jaeger's racès may be considered as variants about this center.

3. D. Weichselbaumi (Weichselbaum, Trevisan). A coccus occurring commonly in pairs of flattened coffeebean shaped cells. Decolorizes by Gram. Develops feebly on ordinary media. Slightly pathogenic for animals. 
Occurs in cerebro-spinal exudate in epidemic meningitis. Exhibits group serum reactions with $D$. gonorrhece.

D. Weichselbaumii has been found in the nose and throat of infected persons and, sometimes, of normal ones. It is not known to occur in animals, but Bertarelli (I906) isolated a form closely resembling it from nodules in the liver and ganglia of the iguana.

The organism described by Class (1899) as the cause of scarlet fever probably belonged in the genus Diplococcus, since it occurred in pairs of "coffee-bean" shaped cells, formed glutinous colonies, and grew badly on ordinary media. It was not, however, distinguished from D. Weichselbaumii by sufficiently definite characters to deserve specific rank.

An organism more or less closely related to D. Weichselbaumii, but exhibiting much higher viability on media than either of the two preceding pathogenic species, occurs not infrequently in normal throats. This is D. catarrhalis, first described and named in print by Frosch and Kolle (I896), by whom credit for verbal description is given to Pfeiffer. It might be considered only a partially saprophytic meningococcus; but the frequency with which it has been isolated, and the fact that it shows distinct serum reactions (Jaeger, I903; Wollstein, I907), seem to warrant its recognition as a distinct type center.

The first careful study of this organism was made by Ghon, Pfeiffer, and Sederl (1902), and these investigators noted the following characteristics. D. catarrhalis is a 
coccus, occurring generally in pairs of "coffee-bean" shaped cells, closely resembling in appearance $D$. gonorrhoee. It occurs at times in tetrads and is always Gramnegative. It is frequently found in small numbers in normal throats, and may be associated with acute inflammatory conditions of the respiratory tract. On agar it forms grayish-white shining colonies with a denser center. It produces scum and sediment in broth and fails to coagulate milk. Its virulence is slight.

Celler (1902), after a careful study, points out the following distinctive marks of $D$. catarrhalis. It grows more profusely on agar than $D$. Weichselbaumii and its colonies have distinct darker centers with whitish shining outer zones. On slants the growth of D. Weichselbaumii is gray, shining, and moist, while that of $D$. catarrhalis is white, dry, and crumbly. $D$. catarrhalis produces acid in glucose and saccharose, and is more distinctly pathogenic than $\dot{D}$. Weichselbaumii. Dunham (I906) observed that organisms of the $D$. catarrhalis type, isolated from the nose and throat, formed sediment when suspended in .75 per cent salt solution and were easily filtered out or removed by a centrifuge; the meningococci, on the other hand, formed a fine suspension impossible to separate by the centrifuge and clogged filters very rapidly. The meningococcus always produced acid in ascites-agar but without coagulation. Some of the forms from the nose and throat coagulated milk, and some failed to produce acid at all. 
On the whole it seems justifiable to recognize a type center of semi-parasitic forms, resembling $D$. Weichselbaumii in most of its characters, but distinguished and defined as follows:

4. D. Catarrhalis (Frosch and Kolle). A coccus occurring commonly in pairs of flattened coffee-bean shaped cells. Decolorizes by Gram. Grows fairly well on artificial media, forming grayish-white colonies with a denser center. Growth on streak, dry and friable. Virulence slight. Occurs in normal throat.

M. albicans-tardissimus, Flügge, M. magnus, Rosenthal, $M$. subtilis, Migula, and other imperfectly described diplococci may perhaps belong to this species.

There is still another type of organism which should probably be referred to the genus Diplococcus, and which apparently links such specialized forms as D. pneumonice with the other less strictly parasitic genera of the Coccaceæ. This is the type previously referred to as Str. mucosus, a form found not uncommonly in the nose and throat, which resembles Streptococcus in the fact that it forms chains and grows easily on artificial media; but agrees with the diplococci in fermentative powers and in the possession of a well-marked capsule.

This capsulated streptococcus was observed by Ortmann (I888) and Besser (1889) and named Str. involutus by Kurth (1893) and Str. capsulatus by Binaghi (1897). Kurth erroneously believed this organism to be the specific cause of foot-and-mouth disease; but his general descrip- 
tion is sufficiently exact to identify it quite clearly. $\mathrm{He}$ described a chain-forming organism, with elongated cells, showing a well-developed capsule under proper cultural conditions, and forming a precipitate in blood-agar (the Libman phenomenon). Kurth's name, D. involutus, must therefore stand for this species, in spite of the fact that other names subsequently bestowed on it are better known.

A similar organism was reported in this country by Richardson (IgOI) and named Str. mucosus by Howard and Perkins (I90I). Another was described by Hlava (I9O2) as Leuconostoc hominis. Longcope (I9O2) in a valuable brief review of the morphological and physiological variations of $D$. pneumonice, maintained that Str. mucosus was merely a variant of the pneumococcus. Buerger (I906) has, however, studied the organism in much greater detail and seems to have established its right to the rank of a specific type. It is distinguished in morphology by the occurrence of capsulated chains in the body fluids, and in cultural characters by the formation of slimy confluent surface growths. According to Buerger it ferments dextrose, dextrin, maltose, lactose, galactose, inulin, levulose, and saccharose, but not dulcit, rhamnose, arabinose, and glycerin. From its fermentation reactions the organism is evidently more closely related to the diplococci than to the streptococci, altho it is in some respects an intermediate form. Neumann (I904) made a careful study of this organism, which he isolated eight times from the nose and throat. He noted 
the frequent occurrence in the chains of oval forms like D. pneumonice. Gram reactions were variable. Milk was coagulated after several days. Schumacher (I906) apparently worked with a somewhat different form, since his cultures failed to ferment lactose; but they were identical with those described by Schottmüller (1903) as Str. capsulatus in respect to their failure to exert hemolytic action. If a capsule-forming streptococcus really exists which shows the fermentation reactions of the genus Streptococcus, it should retain the name of Str.capsulatus of Binaghi. All the observers in this country have, however, worked with $D$. involutus. Thus Park and Williams (I905), like Buerger, found that the culture which they studied produced a green coloration in bloodagar plates and coagulated inulin-serum media. It was distinguished from typical $D$. pneumonice by the fact that it appeared always to ferment mannite and was never affected by specific opsonins. Collins (1905) in a study of agglutinative reactions found the same general relations to hold. Str. mucosus showed distinct group reactions; but exhaustion experiments indicated that its agglutinins were more nearly related to those of $D$. pneumonice than to those of Str. pyogenes.

In view of all these facts this type may be recognized as a valid one, and identified as follows:

5. D. Involutus (Kurth), Winslow. A coccus occurring commonly in chains, the elements of which are grouped in pairs. In the body, and on media, the chains are 
surrounded by a well-marked capsule. Growth on media fairly abundant, moist, and slimy. Ferments monosaccharides, disaccharides, and inulin. Lacks hemolytic power. Shows group serum reactions with D. pneumonice. Occurs on the epithelial surfaces, and in the tissues, of man and the higher animals.

An organism of related type has recently been isolated as the active agent in an epidemic among fowls (Dammann and Manegold, I906); and another form, perhaps allied to it, is the coccus which causes a slimy fermentation of milk, described as Str. hollandicus by Weigmann (1889). In a recent review of the subject, Burri (I904) points out the close resemblance of the slime-producing organism known as Bact. Güntheri to the D. involutus type. M. Sornthalii, Adametz, and Str. sputigenus, Migula, are also apparently forms of $D$. involutus. The peculiar coccus, first described by Johne ( $M$. ascoformans) as producing tumors in horses, growing in botryoidal zooglœa masses within the tissues themselves, is perhaps allied to this group of organisms. 


\section{CHAPTER VI.}

\section{THE GENUS ASCOCOCCUS.}

THE generic name Ascococcus has had a long and varied history. It was first used by Billroth (1874) in his work on Coccobacteria septica, but was not applied by him to a species or a genus. Billroth believed that unlimited pleomorphism prevailed among the bacteria, and his "Ascococcus" was merely the zooglœa form in general, which he thought all bacteria might assume under proper conditions. Cohn (1875), who had a more accurate conception of the constancy of bacterial types, established the name as a generic one. He defined the genus by morphological characters alone, and included in it all forms showing small spherical cells imbedded in lobed masses of gelatinous matter. The species on which he founded the genus, Asc. Billrothii, appeared in his laboratory, forming a zooglœa layer on the surface of an ammonium tartrate culture solution. Under this condition it developed an intense cheesy (butyric acid) odor and produced a strongly alkalin reaction. No coccus has since been found which will grow in such a manner on so simple a medium; and it seems probable that Cohn was actually working with a bacillus rather than a coccus. Many of 
his species of micrococci have since been recognized as really short rods, $-B$. prodigiosus, for example.

The recorded description of Ascococcus, however, was that of a spherical coccus; and an undoubted coccus-form was soon described and referred to this genus. This was the organism which multiplies in saccharose solutions, forming a zooglœa resembling frog spawn, the gelatinous masses growing, under favorable conditions, to the size of a man's head. Cienkowski (1878) first studied this organism carefully and gave it the name Ascococcus mesenteroides. Van Tieghem (I878), a little later, worked on the same form and substituted for Ascococcus the generic name Leuconostoc, in order to emphasize the resemblance between the zooglœa-forming coccus and the blue-green alga, Nostoc.

Both of these names have been set aside by Migula and most recent bacteriologists, on the ground that the production of gelatinous growth masses was a character too unimportant to deserve generic rank; and Asc. mesenteroides has generally been placed with Streptococcus or Micrococcus. It is certainly true that the property of zooglœa formation is specially developed in certain types among many genera of the Coccaceæ. In Diplococcus we have considered a mucus-forming type; in Albococcus we shall find another; and a capsule-bearing form has been described even among the sarcinæ. At the same time the biology of this particular form, Asc. mesenteroides, seemed so unique that we were led in our previous revision 
(Winslow and Rogers, I905) to suggest the restoration of the genus Ascococcus.

We have not had an opportunity of working upon these forms in the laboratory. Further study of the literature, however, has strengthened our opinion that they possess such a peculiar complex of characters as to forbid their inclusion in any other of the established genera. It is clear, in the first place, that Asc. mesenteroides is, in many ways, allied to certain of the Paracoccaceæ. Its cells occur in pairs, or in chains made up of paired elements; and on media which do not contain sugar it grows in small transparent colonies like those of the diplococci or the streptococci. Like organisms of these two genera it produces acid in various sugar media and grows best at temperatures between 30 degrees and 37 degrees. All these properties ally Asc. mesenteroides with the Paracoccaceæ. On the other hand its saprophytic existence and its power of forming relatively enormous gelatinous growth-masses in saccharose and dextrose media, mark it off very clearly from the genus Diplococcus and the genus Streptococcus.

The only resource seems to be the recognition of a distinct genus, which shares the fundamental growthform, temperature optimum, and fermentative powers of the Paracoccaceæ but has also the acquired ability of forming large masses in saccharine solutions. We originally placed this genus with the Metacoccaceæ, in view of its vigorous power of growth and saprophytic habits. More 
detailed studies, which have been summarized in the introductory chapters of this book, have led us to attribute greater importance to fermentative powers; and recent work on the capsule-forming diplococcus, $D$. involutus, makes it probable that Asc. mesenieroides is more closely allied to this type than to any other. On the whole, Ascococcus, tho a saprophyte, seems to be an offshoot of the Paracoccacex; and at the expense of verbal consistency one saprophytic genus must be recognized in an otherwise parasitic group.

The genus Ascococcus has been defined above as follows:

Genus Ascococcus (Cohn) Winslow and Rogers. Saprophytic, growing vigorously in saccharine solutions. Cells in pairs, or in chains of paired elements. In presence of certain carbohydrates large, lobed gelatinous masses of zooglcea formed. Fermentative powers high, acid being produced in dextrose, lactose, and saccharose.

Liesenberg and Zopf in 1892 compared three cultures of Leuconostoc from German and Javanese sugar refineries, and noted certain common characters of considerable interest. The organisms with which they worked appeared able to resist, in the moist condition, a temperature of 80 degrees $\mathrm{C}$. for five minutes. They were favored by the presence of a considerable proportion of calcium chlorid ( 3 to 5 per cent) in the culture medium. They formed gelatinous masses in dextrose media as well as in saccharose solutions. In a recent communication, Zettnow (I907) 
reports that after an examination of $I_{5}$ different strains of Leuconostoc, during a period of ten years, he has been unable to find a single one which possessed the high resistance to heat and to calcium chlorid noted by Liesenberg and Zopf. Two strains which were carefully worked out by Zettnow failed to form zooglœa in dextrose solutions and differed from the type described by Liesenberg and Zopf in certain minor characteristics. They formed an almost invisible growth on potato, failed to grow in asparagin solutions, and did not liquefy gelatin. Their reaction to the Gram stain was apparently negative, isolated cells being decolorized, while those in thicker masses retained the stain. The two strains studied by Zettnow differed only in slight details of colony formation, but one of them failed to agglutinate with the serum of a rabbit injected with the other.

The important differences between the earlier descriptions and those of Zettnow appear to lie in the high resistance to moist heat and to calcium chlorid, and in the zooglœa formation in dextrose solutions, recorded in the former. In the absence of evidence confirming the observations of Liesenberg and Zopf these points may be left in abeyance. In other respects a single species of Ascococcus seems to be indicated. This may well bear the name of Cienkowski's original type, Asc. mesenteroides. In view of the published descriptions of various observers it may be characterized as follows:

I. Asc. mesenteroides (Cienkowski). A coccus occuring commonly in pairs, or chains of paired elements. 
On media not containing sugar, produces faint translucent colonies; on media containing saccharose, forms large, lobed masses of zoogloa. Produces acid in dextrose, lactose, saccharose, and dextrin. Develops best between 30 and 37 degrees. In zoogloa stage highly resistant to dry heat.

It is possible that other imperfectly described organisms, which produce slimy fermentations in wine and milk, may belong to the genus Ascococcus. Leucocystis cellaris, Schröter, and the closely related Leuconostoc Lagerheimii, isolated by Ludwig (Migula, I900) from decayed wood, may be distinct species. It is likely that intermediate forms will be found between Asc. mesenteroides and D. involutus; and the relations of these two types can only be definitely settled by comparative study. $M$. mucilaginosus of Migula, a slowly liquefying form, is a peculiar organism, allied to one or the other of them. 


\section{CHAPTER VII.}

\section{THE GENUS STREPTOCOCCUS.}

The genus Streptococcus has been defined by its parasitic life, the occurrence of the cells in chains, positive reaction to the Gram stain, faint effused growth on media, high fermentative power for most carbohydrates, but not for inulin, and the general failure to liquefy gelatin or reduce nitrates. The single cells may be elongated or lance-shaped but are more often spherical, or even flattened at right angles to the chains in which they occur. They may appear in long chains of a hundred or more elements, or in pairs and short chains of four to six cells. Chains are best developed in broth cultures; but some strains display the tendency to chain formation much more markedly than others. Chains are frequently made up of successive pairs of flattened cells, - probably as the result of active division. Double chains and irregular plate-like groups sometimes appear. Cocci isolated freshly from the mouth are particularly apt to show this grouping, and occasionally exhibit elongated rod-like cells. Large cells sometimes appear in chains of smaller ones. Vincent (1902) has even described a branching streptococcus which he isolated from a pleuritic exudate, and which he records as retaining its habit of pro- 
ducing dichotomously branching chains during repeated cultivations. This author believes that differences, both in chain formation and in the manner of growth in broth, are markedly influenced by the reaction of the medium. He states that a streptococcus which forms long chains and shows sediment in acid broth will grow in short chains and show a uniform turbidity if the broth be made slightly alkalin. The Gram stain is usually positive with the streptococci, especially in cultures freshly isolated from the body. Cultivation in artificial media produces more variable results.

The streptococci are essentially parasitic forms. Their habitats are the various surfaces of the animal body, from which they penetrate the tissues in acute disease. Their growth on solid media is always feeble. On potato it is generally so sparse as to be invisible. Cultures die out very rapidly in successive transfers. Cultivation under anaerobic conditions is often more successful than in the presence of air. In broth, cultures grow well for a few transfers, producing turbidity and, later, sediment if the growth is active, or sediment alone if it is less vigorous. Sugars are favorable for development, and are generally fermented; milk is coagulated by some strains but not by all; other biochemical reactions are generally negative. The virulence of the streptococci is extremely variable. When grown on culture media they rapidly become nonpathogenic. Repeated passage through animals, on the other hand, may produce so high a virulence that a very 
minute dose will kill a rabbit in a few hours. The same culture "may produce at one time merely a passing local redness, at another a local suppuration, at another a spreading erysipelatous condition, or again a general septicæmic infection, according as its virulence is artificially increased" (Muir and Ritchie, I903). This extreme variability explains the association of closely related streptococci with such widely different diseases of the human subject as erysipelas, phlegmons and spreading inflammation, joint diseases, endocarditis, anginas, puerperal infections, and general septicæmias.

The genus has been defined above as follows:

Genus Streptococcus (Billroth) Winslow and Rogers. Parasites. Cells normally in short or long chains (under unfavorable cultural conditions, sometimes in pairs and small groups, never in large packets). Generally stain by Gram. On agar streak, effused translucent growth, often with isolated colonies. In stab culture, little surface growth. Sugars fermented with formation of large amount of acid. Generally fail to liquefy gelatin or reduce nitrates.

On the whole, the genus Streptococcus is well marked and easily distinguished. Its relation to Diplococcus, into which it grades most closely, has been somewhat fully discussed already. It is distinguished from the rest of the Paracoccaceæ, on the other hand, by its characteristic morphology and sparse growth on artificial media. Three of Cohn's original species of micrococci, M. Vaccinere $M$. diptericus, and $M$. septicus, were probably representa- 
tives of this genus (Cohn, I875); but his descriptions are too incomplete for identification. The first clearly defined species was the Streptococcus erysipelatos of Fehleisen (1883). In the next year Rosenbach (1884) described a similar organism as the cause of wound infections, under the name Str. pyogenes. Similar streptococci have been found in a multitude of pathological conditions, - in diseases of the skin and of the joints, in sore throats and lung infections, in intestinal and puerperal diseases, in secondary infections, and in general septicæmias; and others have been described as the specific agents in scarlet fever, small-pox, and rheumatic fever. Some bacteriologists have held that each of these types was a specific entity; others have maintained with equal vigor that all were variants of a single unit-form.

Streptococci are also frequently associated with diseased conditions in animals, and have been studied with special care by veterinarians in connection with inflammations of the cow's udder. The large volume of literature upon this subject has been admirably summarized by Steiger (I904), with the addition of original observations of his own upon a considerable series of organisms. None of the cocci of bovine disease appear to differ from organisms previously described from the human body or from air, under other names. Streptococcus mastitidis sporadice and Str. agalactice contagiosce are forms of Str. pyogenes; and here, as in the human subject, long-chained and short-chained varieties have 
been recognized. Staphylococcus mastitidis characterized by whitish growth, acid production, and liquefying power, is evidently a form of the genus Albococcus probably Alb. pyogenes. The various species of the genus "Galactococcus" of Guillebeau are only well-recognized forms of the genera Aurococcus, Albococcus, and Micrococcus.

Staeheli (I904) has recently pointed out the great variability exhibited by the streptococci associated with diseases of the cow's udder (Str. agalactia contagiosa) and their close relation to Str. pyogenes. Baruchello (I905) was unable to distinguish the streptococcus normally found in the intestine of the horse from Str. pyogenes; and to go still farther afield, recent observers attribute a common silkworm disease to a form differing only in its failure to coagulate milk (Sartirana and Paccarano, I905). The latter is perhaps the Micrococcus bombycis of Pasteur (Cohn, I875).

A third class of workers, the dairy bacteriologists, approaching the subject from another standpoint, have isolated the same group of organisms. The streptococcus which is now clearly recognized as one of the commonest "lactic acid organisms" in milk, and which was described by Kruse (I903) as Streptococcus lacticus, shows no valid specific differences from Str. pyogenes. Hölling (I905) studied a number of races of streptococci obtained from sour milk and concluded, in spite of slight differences in acid production and other characters, that all were of the 
same species, - a species which includes Streptococcus lacticus, Bacterium Güntheri, and Bacterium lactis acidi, and is identical with Streptococcus pyogenes. He notes that cultures from fæces, with feebler growth and acid production, showed a higher pathogenicity. Heinemann (I906) has recently shown that the organisms which ordinarily cause the lactic acid fermentation of milk are two, a bacillus and a streptococcus; and that the latter, Str. lacticus of Kruse, is identical in morphological and cultural properties with the streptococci isolated from the normal and diseased human body. The same observer has found (I907) that pathogenicity offers no distinction between Str. lacticus and Str. pyogenes. It appeared in his experiments that five passages through rabbits would produce a high degree of virulence in the former organism.

Numerous streptococci isolated from cheese by Henrici (I897) are indistinguishable from Str. pyogenes according to the author's descriptions. Macchiati (1899) found similar organisms in macaroni, as did Prescott (I906) on various grains, including wheat, buckwheat, rye, barley, and oats. It is possible that these organisms, like B.coli, may live in a semi-parasitic fashion on plants, as well as on animals.

In none of the investigations cited did the streptococci isolated from other sources differ in any constant way from those found on or in the human body. In cultures of either human or animal origin differences existed; but 
these differences were too slight to offer any satisfactory basis of classification. Miguła (I900) listed forty-seven species under the genus Streptococcus, and Le Gros (1902), sixty-three; but most of these "species" are identical in all the important characters described. Minute differences in the appearance of colonies, dryness, granulation, outgrowths from the edges, etc., are given diagnostic value in certain cases; but these are to be attributed mainly to variations in the conditions of cultivation. Such differences in vitality as are shown by the failure to grow upon potato or on gelatin at 20 degrees also occur; but these can certainly not be considered as of systematic value. One or two fairly definite types have, however, been distinguished by differences in morphology, and in the character of growth in broth.

Lingelsheim (189I) distinguished two species, Str. longus and Str. brevis, the former characterized by long chains and large flocks in broth, the latter by short chains and diffuse turbidity. Str. brevis was generally not pathogenic, liquefied gelatin slightly, and exerted a strong reducing action on certain dyestuffs. This distinction apparently corresponds to a real division between two types of streptococci. Vincent and others have shown, however, that both morphological characters (the length of chains) and cultural characters (turbidity in broth) are markedly influenced by the reaction of the medium; and even under the same conditions the same strain may exhibit wide variations. 
Kurth (I89I) described, under the name Str. conglomeratus, a type which he found often associated with scarlet fever, and which was characterized by the formation in broth of compact flakes of sediment composed of tightly woven masses of chains, and by a high pathogenicity.

Behring (1892) recognized four varieties of Str. longus, the first yielding diffuse turbidity in broth, the second forming a soft, slimy sediment, the third producing scaly flocks of sediment (Kurth's Str. conglomeratus), and the fourth growing in balls made up of long chains tending to cling to the walls of the tube. This fourth variety of Behring's was described by Roscoe and Lunt (I892) as Str. mirabilis. Behring's pupil, Knorr (I893), later showed that these differences are highly variable; and produced from the same culture two races, differing markedly in growth and in virulence, by successive cultivations and animal inoculations. Pasquale (1893) a year or two later made an elaborate comparative study of the streptococci, using for his material thirty-eight different strains, representing almost all the forms which had at that time been described. He confirmed the view that the most virulent streptococci are in general those which form long chains in broth, tho all long-chained forms are not necessarily virulent; and finally divided the organisms studied into four classes: the short saprophytic streptococci (from fæces and the alimentary tract), the long chain-formers of low virulence, the long-chained virulent forms, and the short, highly infectious forms (the diplococci of pneumonia and 
similar conditions). Each of these four groups contained two subtypes, one growing at low, the other only at higher temperatures.

With the introduction of the newer methods of studying physiological immunity, it was hoped that the relations of the various pathogenic and non-pathogenic streptococci might at last be elucidated. The practical importance of a clear differentiation of these forms was greatly enhanced by Marmorek's introduction (I895) into therapeutics of the anti-streptococcic serum. This investigator, (Marmorek, I902, a), was able to obtain a very strong streptococcus toxin by first cultivating his organism in serum to which the leucocytes of an immune guinea pig had been added, and then growing it in broth containing leucin and glycocoll, which he found favored the continued production of the toxin. The antitoxin corresponding to the poison, he believed, could be used in all infections in which streptococci were concerned. He considered the unity of the streptococci of man to be demonstrated by studies of serum reactions, of hemolytic activity, and of the power to render a medium unsuitable for the development of another strain (Marmorek, I902, b). In all these respects, however, his own records show marked quantitative variations between different races.

At about the same time other observers were attempting to identify special strains of streptococci as the causative agents of specific diseases, notably scarlet fever, smallpox, and rheumatic fever. Baginsky and Sommerfeld 
(Baginsky, I896; Baginsky and Sommerfeld, I900; Baginsky, I902; Sommerfeld, I903) and their followers believed that scarlet fever was caused by a specific streptococcus; and their attempts to treat this disease by anti-streptococcic serum attracted wide attention. Moser (I902) and Moser and v. Pirquet (I9O2), by injecting horses with streptococci isolated from cases of scarlet fever, were able to prepare immune sera which showed a certain group agglutination with streptococci from similar sources, and which when administered to human beings appeared to exert a favorable effect upon the course of the disease. Other observers were less successful. Neufeld (I903) concluded that rabbits immunized against one streptococcus would exhibit an equal immunity and equal agglutinative power against other streptococci from various sources. Organisms from scarlet fever showed no characteristic relations. Weaver (I904) also found no constant group serum reactions, which would separate the streptococci of scarlatina from those of other pathological conditions.

The isolation of streptococci from rheumatic lesions was reported by various observers prior to I900; and in that year a number of English investigators became convinced that an organism of this group bore a definite causative relation to the disease. Poynton and Paine (I900) described a streptococcus which they had isolated from eight successive cases, apparently a small Gramnegative form of Str. pyogenes. This organism was 
named $M$. rheumaticus by Beaton and Walker (1903). It was said to differ from typical Str. pyogenes in four points, as follows: it changed the red color of blood-agar to rusty brown, it grew well in filtrates from cultures of ordinary pyogenic cocci, it grew in highly alkalin media, and in such media produced considerable quantities of acid. Inoculation of small doses into rabbits produced acute arthritis. Walker and Ryffel (I903) state that this organism, when grown in alkalin broth, produces a considerable amount of formic acid, which they believe to offer another differential test for separating it from Str. pyogenes. The production of formic acid by this streptococcus was considered by them as of special interest in view of the presence of this acid in the urine of rheumatic patients. Poynton (I904) later reported a new isolation of $M$. rheumaticus, with successful animal inoculations; and Beattie (1906), in a recent paper, strongly maintains that the results obtained by injecting this form into animals are very different from those which follow the injection of Str. pyogenes. On the other hand, Cole (I904) and others deny the validity of these conclusions and offer evidence that streptococci from various sources produce, when inoculated into animals, the symptoms held to be characteristic of $M$. rheumaticus. Furthermore, in a long series of cases no organism could be isolated by these observers from rheumatic lesions.

With regard to smallpox, somewhat similar observations have been made, altho evidence is against the 
supposition that the secondary infections, characteristic of this disease, are due to any specific streptococcus. Here, as in scarlet fever and rheumatism, the streptococci isolated from different cases of the same disease may often show a distinct group relationship in their reaction to sera. This has been shown for smallpox by De Waele and Sugg (1903) and others. The phenomenon is, however, a variable and uncertain one. Perkins and Pay (I903) studied sixteen cultures of streptococci from variola, and found several distinct varieties, varying in vigor of growth on media, in action on lactose in milk, in formation of turbidity in broth, and in agglutination reactions.

It seems certain that group serum reactions are frequently manifested by the streptococci associated with specific pathological conditions. Even Meyer, one of the strongest adherents of the "Einheit der Streptokokken," found , significant differences between the behavior toward immune sera of the common pyogenic streptococci and of the forms isolated from rheumatism and scarlet fever (Meyer, I902). These group differences, however, affect only those highly variable qualities and not the more constant morphological'and biochemical properties. Even with regard to serum reactions themselves the supposed specific differences are by no means universal. Aronson (I903) gives a good summary of the data previously published on this point and adds the results of a careful study of 27 different strains isolated from scarlet fever, rheumatism, septicæmia, etc. Neither agglutination 
nor immunization experiments showed constant group reactions, altho the cultures exhibited marked individual variations.

Fischer (1904) made a careful review of the literature of this subject, and carried out a considerable series of experiments on the agglutination of various strains of streptococci. Like the observer just cited, he found that each strain showed a specific reaction with the serum of the animal into which it had been injected, a somewhat less sharp reaction with other strains, and no agglutination with still others. The forms showing close similarity in agglutination were not, however, those most closely related in origin and pathogenicity. Kerner (1905), after exhaustive investigations, came to the conclusion that serum reactions among the streptococci were so erratic as to be valueless in systematic work. Nedrigailow (I906) has recently reviewed the evidence for constant specific types in scarlet fever and smallpox, and concludes, by analogy with the serum reactions of other pathogenic streptococci, that the special properties of the streptococci in these diseases are only temporarily acquired characters, developed in response to their peculiar environment.

The hemolytic power possessed by the pathogenic streptococci is another of the subtle properties developed in direct response to the biochemical conditions of their host. Schottmüller (I903) used this character as a differential test, noting that, besides a zooglœa-forming organism (D. involutus), two types of streptococci may be 
distinguished by growth in blood media. The first, which he called Str. longus pyogenes seu erysipelatos when grown in blood-broth, changed its color to a burgundy red, and on blood-agar its colonies were surrounded by a clear zone. The second, Str. mitior seu viridans, produced a brownish color in blood-broth, and on bloodagar formed greenish colonies without hemolysis. The former were generally more pathogenic, occurred in longer chains, produced sediment instead of turbidity in broth, and more generally failed to coagulate milk. The capsulated streptococcus and $D$. pneumonice behaved like Str. mitior in blood-agar. Rieke (I904) studied, with some care, the hemolytic power of the streptococci, which underlies Schottmüller's test; and found that twenty-five cultures, all of the Str. longus type, produced essentially the same change in blood-bouillon. All dissolved the blood cells and set free oxyhemoglobin, giving the burgundy-red coloration, which, according to Schottmüller, is characteristic of Str. longus; later, in all cultures, the oxyhemoglobin was changed to neutral methemoglobin, giving the brownish-red color supposed to be characteristic of Str. mitior.

Boxer (I906) studied the behavior of a considerable series of cultures of diplococci and streptococci on bloodagar. Thirty-six of the 47 streptococci showed characteristic decolorized areas around the colonies, due, as shown by microscopic examinations, to hemolysis. None of the others showed the green color of Str. mitior. 
Ruediger (I906, a), still more recently, has shown that this green coloration is due to the action of the cocci upon the muscle-sugar present. In sugar-free media the action disappears; and, on the other hand, in glucose blood-agar he found that Str. pyogenes produces a green coloration. Which type of colony will appear depends then on the relative amount of action on sugar and blood cells respectively. Kerner (1905) in his investigations found that the hemolytic power was strong in streptococci freshly isolated from the body, but decreased rapidly on artificial media.

On the whole, the much debated question of the "Vielheit" or "Einheit" of the streptococci received no satisfactory solution from the methods of experimental immunity. These organisms remained as before, - a more or less closely related group made up of an indefinite number of varieties.

Two recent revisions of the genus have been made by Le Gros (1902) and v. Lingelsheim (1903). Le Gros, after reviewing the evidence from morphology and the biochemical tests, recognized ten groups. Group (I) included streptococci showing typical chains, long or short, producing or failing to produce turbidity in broth, and non-pathogenic; group (2) differed in having pathogenic power; group (3) included motile; and group (4) non-motile streptococci; group (5) was defined by negative reaction to the Gram stain; group (6) by abundant growth on potato; group (7) included liquefying forms; 
group (8) was marked by the occurrence of the cells in pairs; group (9) by the presence of a capsule; and group (10) by the arrangement of the cells in plates rather than chains. Even without taking into account the overlapping of the various groups, this classification is scarcely adequate. Virulence and growth on potato are such exceedingly unstable characters as to be of little diagnostic value in this genus. Motility is of extremely doubtful occurrence; no motile streptococcus has been recorded by any good observer during the recent period in which the methods of bacteriology have developed to approximate accuracy. The last three groups include organisms which probably belong to other genera.

Lingelsheim (I903), in a somewhat less arbitrary fashion, divided the genus into seven species as follows: (I) Str. longus, characterized by long chains, positive Gram stain, small colonies on agar, and variable pathogenicity; (2) Str. brevis pharyngis, differing in showing short chains, no pathogenicity, and feeble viability in cultures; (3) Streptococcus of Étienne, chains of slender cells decolorizing by Gram; (4) Streptococcus of the saliva, non-pathogenic, short-chained form, capable of growth on potato; (5) Str. brevis liquefaciens, differing from No. 4 in decolorizing by Gram and liquefying gelatin; (6) Str. coli gracilis, differing from No. 5 in showing longer chains with a tendency to the formation of tetrads; (7) Leuconostoc, the zooglœa-forming type.

In this classification the use of several characters in 
correlation serves to fix certain groups of a fairly satisfactory sort. The first two species, the long-chained virulent form and the short-chained avirulent form, have been referred to above as natural types. As to the fourth species, it seems doubtful, as we have said, whether the viability on potato is not too slight and fluctuating a character for fixing a species. Species 5 and 6 are certainly only minor variants of the same liquefying type. Species 7 , according to our view, does not belong in this genus.

So the case rested in I903; and v. Lingelsheim concluded at the end of his monograph that only the future could decide as to the unity or plurality of the genus. In the most recent French medical treatise, Widal (I906) emphatically maintained the inadequacy of any of the differential methods previously proposed for the differentiation of the various pathogenic and non-pathogenic streptococci of the body. One hundred and twenty-two different strains studied by him showed no constant differences in biological characters. Those obtained from pathological conditions generally produced erysipelas in rabbits, while those from the normal mouth did not; but these pathogenic powers Widal recognized as wholly transitory.

Two innovations in systematic bacteriology were required in order to clear up this vexed question, - a detailed comparative study of fermentative powers and a statistical analysis of the results obtained. The first of 
these points, the action of the streptococci on carbohydrates, which has proved of such great diagnostic value, was first worked out by Dr. Mervyn H. Gordon of the Medical Office of the English Local Government Board.

Page (1899), eight years ago, made a quantitative study of the acid produced by twenty-one different streptococci in dextrose, lactose, and saccharose. All produced acid; and Dr. Page concluded that the organisms could not be separated by a quantitative study of fermentative power. His tabulated results are of interest, however, as showing several of the types worked out later by Gordon. Some study of fermentative power has also been made more recently in connection with the comparative studies on the diplococci. Thus Ruediger (I906, b), in a study of the streptococci from normal and scarlatinal throats, found two types, one fermenting mannite and the other failing to do so, as well as a group of organisms intermediate between the streptococci and the diplococci.

The comparative study of fermentative power on a large scale we owe, however, to the striking investigations of Gordon. His first studies along this line (Gordon, I904) were made in the attempt to develop a test for mouth bacteria, to be used in estimating air pollution. He found that the most abundant organisms in the normal mouth were cocci, and that the most ubiquitous of all were the streptococci. The streptococci found in the mouth showed the usual characters of Str. pyogenes, varying in the length of chains (Str. longus, Str. medius, and Str. 
brevis) and in the tendency to form coherent growths in broth (Str. conglomeratus). Of seventy-seven cultures, sixty coagulated milk and twelve more produced acid in it. All fermented dextrose. Neutral red, however, was reduced only by organisms of the Str. brevis type, and this appeared to offer a valuable means of differentiation. Of twenty-two cultures isolated from the air, six resembled the common mouth-type (Str. brevis), but the other sixteen differed in failing to reduce neutral red. In an addendum to this paper, Gordon noted that experiments with twelve cultures, in regard to their acid production in various sugars, indicated that all fermented dextrose, levulose, maltose, and galactose, and none altered dulcitose or starch. Lactose, saccharose, inulin, raffinose, and mannite, on the other hand, were acted upon by some strains and not by others, and their use for diagnostic purposes seemed promising.

In the next year Gordon (I905) worked out the fermentative powers of the groupin a more comprehensive manner. He first noted the effect of ten different cultures of streptococci upon thirty-five different organic compounds. Many of these substances were affected by all ten strains, and others by none of them. The details, which are full of suggestiveness in relation to the biology of these bacteria, are given in the original paper. Seven carbohydrates were finally selected as of special differential value, being acted upon by some strains but not by others. They included two disaccharides, saccharose and lactose; 
one trisaccharide, raffinose; one polysaccharide, inulin; two glucosides, salicin and coniferin; and one alcohol, mannite. To these seven reactions, the clotting of milk and the anaerobic reduction of neutral red were added, forming what have since been known as "Gordon's tests." Applying these nine tests to three hundred different strains of streptococci from normal saliva, forty-eight different combinations of reactions were distinguished. All the streptococci from saliva were alike in forming acid in saccharose, and in failing to produce any in mannite. Two types occurred with marked frequency; in both, positive reactions were obtained with saccharose and lactose, milk was clotted and neutral red reduced, while no acid was formed in inulin, salicin, coniferin, or mannite. One type fermented raffinose and the other did not. Short chains were generally found. Twenty streptococci from pathological conditions gave somewhat similar reactions, but in three cases mannite was fermented. Twelve cultures from dust, air, and other sources showed only one positive reaction to raffinose, four negative reactions to saccharose, and six positive reactions to mannite.

Houston(I905, a)applied Gordon's tests to three hundred strains of streptococci from fæces, and found forty different combinations of characters. A large proportion of his strains fermented the disaccharides, saccharose and lactose, and the glucoside, salicin. Only a third, however, attacked the trisaccharide, raffinose, and a quarter the alcohol, mannite, while less than 5 per cent fermented 
the polysaccharide, inulin. Three-quarters of the cultures produced diffuse turbidity in broth, and about the same proportion showed short chains under the microscope. One hundred and seventy-two streptococci isolated by the same observer (Houston, I905, b) from cow's milk showed somewhat different average characters. As compared with the fæcal strains, a larger proportion of the milk streptococci had medium or long chains (3I per cent), and a smaller proportion (only 46 per cent) formed diffuse turbidity in broth. Positive reactions were rarer in salicin (6o per cent), in raffinose ( 19 per cent), and in neutral red broth (20 per cent); but more common with inulin (2I per cent), lactose (97 per cent), and litmus milk ( 70 per cent). In the next year Houston (I906) studied one hundred streptococci from cow dung and found marked differences between their properties and those of the human intestine in three reactions. Mannite was fermented by 24 per cent of the human cultures and by none of the bovine strains; raffinose was fermented by 32 per cent of the human and by 74 per cent of the bovine cultures; neutral red was reduced by 39 per cent of the human strains and by none of those from cow dung.

Gordon's tests completely demolished the "Einheit" of the streptococci. As he and Houston left the matter, however, the group was in a state of "Vielheit" so complex as to be appalling. The enormous series of variants which the fermentation tests revealed required 
comparison and correlation, before their type centers could be discovered; and fortunately this task did not wait long. Andrewes and Horder (I906) have applied the statistical method to the species of this group; and by a vast amount of work, carried out from a sound biological viewpoint, have at last brought order out of chaos. The descriptions of twelve hundred strains of streptococci were available to these authors - three hundred isolated by Gordon from the mouth, three hundred of Houston's from fæces, one hundred and seventy-two of Houston's from milk, two hundred of Gordon's from air, and two hundred of their own from diseased conditions (the last including pneumococci as well). These records were analyzed with a view to determining which of the reactions were of fundamental, and which of subsidiary, importance. Given strains were tested first as to the constancy of their characters; and the investigators concluded that altho the power to ferment salicin and mannite was sometimes lost in cultivation, the constancy of a given strain was on the whole surprisingly great. When they proceeded to attempt the correlation of the various characters, however, their results at first appeared disheartening. The number of types was bewildering, each variety being connected with others by innumerable transitional forms.

A study of the numerical frequency of various combinations showed, however, that certain types were of much more common occurrence than others. Andrewes and Horder's picturesque comparison of the distribution 
of these forms to a mountain range, with peaks and connecting valleys between, has been quoted in an earlier chapter. The essential point in their work was the establishment of the fact that type centers occur among the streptococci, about which most of the cultures found in nature group themselves more or less closely. Furthermore these types are in most cases associated with definite habitats, - a strong point in favor of their systematic significance. The division between types is not, of course, a sharp one. The centers to which Andrewes and Horder have given names shade off into each other by imperceptible gradations. Yet "this indefinite state of affairs actually represents the condition found in nature." The scheme outlined "fairly represents the actual medley of types seen in nature, and the way in which, by the processes of evolution, nature herself is sorting out the medley." "Some such conception of the streptococci as we have set forth is preferable to the idea that they are all one kind or that they present a hopeless chaos."

With this preamble, Andrewes and Horder proceed to define seven species of streptococci (including the pneumococcus) as follows:

I. Streptococcus equinus (Andrewes and Horder). This type of streptococcus appears to be characteristic of the intestine of the herbivora. It is abundant in horse dung and is the commonest form in the air of London. It forms chains of medium length, grows feebly, if at all, at 20 degrees, and ferments saccharose and the glucosides 
(salicin and coniferin), but not lactose, raffinose, inulin, or mannite. It fails to clot milk or to reduce neutral red.

2. Streptococcus mitis (Andrewes and Horder). This type is found most commonly in human saliva and faces, but is not as a rule associated with disease. It is short-chained, grows well on gelatin at 20 degrees, and acidifies milk without clotting. It ferments lactose as well as saccharose and salicin, but gives a negative reaction to the other tests.

3. Streptococcus pyogenes (Andrewes and Horder). This type represents the highest parasitic development of the group, being rarely found except in association with definite pathological conditions. It occurs in long chains, usually growing in woolly masses at the bottom of a clear broth. It grows well on gelatin at 20 degrees. It is actively hemolytic, but does not form hydrogen sulphid in broth culture. It strongly acidifies milk, but never clots it, nor does it reduce neutral red. The usual positive reactions with Gordon's tests are saccharose, lactose, and salicin. It is highly pathogenic for animals.

4. Streptococcus salivarius (Andrewes and Horder). This type is the commonest form in the mouth, altho it is also found in the intestine. It is a shortchained form which usually renders broth uniformly turbid. Its growth on gelatin at 20 degrees is variable. It clots milk and reduces neutral red and ferments saccharose, lactose, and raffinose. 
5. Streptococcus anginosus (Andrewes and Horder). This type is a pathogenic long-chained form, allied in other respects to Str. salivarius, and bearing to it much the same relation which Str. pyogenes bears to Str. mitis. It occurs most commonly in cases of scarlatinal and other forms of sore throat. It is long-chained and produces a flocculent deposit in broth. It generally fails to grow on gelatin at 20 degrees, and is markedly hemolytic. Like Str. salivarius, on the other hand, it clots milk, reduces neutral red, and forms acid in saccharose, lactose, and raffinose.

6. StReptococcus facalis (Andrewes and Horder). This type is specially characteristic of the human intestine. It is short-chained and renders broth uniformly turbid. It grows readily at 20 degrees and forms sulphureted hydrogen in broth cultures. It has no hemolytic power and little virulence, but produces a positive reaction to all of Gordon's tests except raffinose and inulin. That is, it clots milk, reduces neutral red, and ferments saccharose, lactose, salicin, coniferin, and mannite. The mannite reaction is specially characteristic of this intestinal type.

The seventh of Andrewes and Horder's types is the pneumococcus, which we have placed in the genus Diplococcus. It is characterized by the presence of a capsule under proper cultural conditions. It occurs in pairs or short chains. It is virulent, and fails to grow on gelatin at 20 degrees. It fails to act on neutral red, or to ferment 
mannite and the glucosides, but does form acid in saccharose, lactose, raffinose, and inulin, and generally clots milk.

All these species are of course type centers only. Variants "by suppression or addition" of certain characters, as Andrewes and Horder express it, are found in each group. They describe a considerable number of such variants. The concentration of strains about the seven combinations of characters to which they have given names seems, however, to mark them off distinctly as the principal centers about which the members of the genus are varying. They appear to deserve specific rank, in the only sense in which the term "species" can be applied among the bacteria.

It is somewhat unfortunate that these authors did not conform to the accepted custom of biological nomenclature, and adopt for their types the names by which similar organisms have previously been described, even if the earlier accounts were incomplete. They acknowledge, for example, that their third type was first described as Str. erysipelatos by Fehleisen, but prefer Rosenbach's name, Str. pyogenes, on account of its wider use. One of their short-chained forms should have kept the name Str. brevis of von Lingelsheim, and another might well have been identified with Str. mitior of Schottmüller. Andrewes and Horder, however, preferred to give new names to their types; and since they have done so their names must stand, as they, for the first time, have described streptococcal types with sufficient clearness 
and detail to make them definitely recognizable. The names Str. erysipelatos, Str. longus, and Str. brevis must therefore be abandoned as insufficiently characterized. Str. conglomeratus Kurth and Str. mirabilis Roscoe and Lunt are apparently only varieties of Str. pyogenes or Str. anginosus. The intestinal forms, Str. enteritis, Hirsh, and Str. enteritis, Libmann, as well as the streptococci associated with the souring of milk, Str. lacticus, Kruse, $M$. acidi lactici, Marpmann, $M$. pseudocerevisice, Migula, and others, are indistinguishable by their published descriptions from Str. fecalis. The streptococci isolated from diseased conditions in horses and cattle, Str. equi, Schütz, Str. mastitidis, Guillebeau, and $M$. brevis (Babes), Migula, for example, have no distinct characters to warrant their recognition. The same is true of the various urine-fermenting forms, described by Rovsing and others, and of the numerous species from cheese, defined by Henrici according to the size of the individual cells and the appearance of gelatin colonies. These organisms may probably be classified under one or other of Andrewes and Horder's types. To which type they belong, can only be determined by study of the fermentative power of a considerable series of strains, isolated from the various sources in question.

Certain of the older bacteriologists described streptococci producing abundant chromogenic surface growths (red or yellow). No such forms have been recorded in recent years; and it seems likely that erroneous conclusions 
were drawn from contaminated cultures, or from the fact that short bacilli were mistaken for cocci. It is possible that Str. bombycis (Béchamp), Cohn, and Str. Pastorianus, Krassilschtschik, and M. lardarius, Krassilschtschik, all three isolated from the bodies of diseased silkworms, may represent a distinct type; and Migula's record of the occurrence in great numbers of a streptococcus, Str. Sphagni, in swamp water suggests the possible existence of saprophytic forms. Neither of these types has been worked out with fullness, however.

The streptococci described in connection with various specific diseases seem to possess an equally slight claim to recognition as distinct types. Andrewes and Horder found no evidence of a specific streptococcus acting as the causative agent in scarlet fever. Their investigations tended also to discredit the organism described under the name of $M$.rheumaticus as the specific agent in rheumatic fever. Two supposedly typical cultures which they examined were examples of Str. salivarius and Str.facalis, respectively.

The Str. scarlatince, described by Gordon (I90I), is distinguished from typical Str. pyogenes by the formation of conglomerate masses in broth, and by the frequent occurrence of rod-like forms in its chains. This is evidently the same organism described by Kurth (I89I) as associated with scarlet fever, under the name Str. conglomeratus. It does not appear to form a distinct species.

The organism described by Bruce (I893) as the causa- 
tive agent in Malta fever was thought by him to be a coccus, and in its general cultural relations it resembles Str. pyogenes. Babes (1903) claims that the "M. melitensis" of Bruce is a short bacillus. The biochemical properties of the organism, and the relations of its serum reactions to those of other forms, are not sufficiently worked out to make its position clear.

If the pneumococcus is placed in the genus Diplococcus, six of Andrewes and Horder's types remain as species of the genus Streptococcus. It is probable that further study of biochemical properties may reveal other important centers of variation. In a suggestive paper, Sieber-Schoumoff (1892) pointed out two such characteristics, which might be of value in the differentiation of streptococci. She found that certain strains, when cultivated in sugar broth, formed optically inactive lactic acid, while others produced the levo-rotary compound. Of the latter group, some cultures showed the power of forming salicylic acid in broth containing salol. Hashimoto (I90I) has described a streptococcus and a micrococcus (apparently a strain of Albococcus pyogenes) both of which formed pure dextro-rotary lactic acid from milk-sugar. From Hashimoto's review of previously described organisms, it appears that the production of dextro-rotary acid has been recorded among the cocci in four cases, and that of levo-rotary acid only once, by Leichmann.

In our own work we attempted to divide the streptococci 
into groups by their quantitative power of fermenting dextrose and lactose. Sixty-one cultures showed an average acid production of $\mathrm{I} .9$ per cent normal in dextrose broth and I.4 per cent normal in lactose broth. The mode in each case was near the lower end of the scale, indicating that the strong acid-producers are "variants by addition" from less active types. One point which deserves further investigation is an apparent correlation between high acid production and a positive reaction to the Gram stain. In our small series of cultures it was apparently true that these two properties were definitely associated, negative Gram reactions being observed only with cultures of slight acid-producing power.

There is one type of streptococcus, besides the six described by Andrewes and Horder, which seems at present sufficiently well marked to deserve specific rank. The best basis for species - and the only satisfactory basis is a study of the frequency with which various types occur, specific names being given to the centers of variation. When, however, some strains exhibit a highly peculiar characteristic we may recognize a provisional species, pending comparative studies, if the property be sufficiently definite and of fairly common occurrence. Such a provisional specific type seems warranted in the case of the liquefying streptococci.

A slight liquefying power has been noticed, again and again, in exceptional strains of streptococci, (Str. Brightii, Trevisan, Str. enteritidis, Escherich, Str. Bonvicini, Ches- 
ter, Str. Fischeli, Chester, Str. carnis, Chester). Escherich (I886) described a form which liquefied quite actively, under the name of Str. coli gracilis. It was characterized by the very small size of the cells; it failed to coagulate milk and did not liquefy blood serum. To illustrate the confusion which has arisen from trinomials, it may be noted that this organism was called Str. gracilis by Lehmann and Neumann (1896), and Str. coli by Chester (I90r). Str. septicus (Babes), Chester, and Str. liquefaciens, Sternberg, are closely related forms. An interesting streptococcus, described by MacCallum and Hastings (I899) as Micrococcus zymogenes, showed the same characteristics as Str. gracilis (small size, liquefaction of gelatin), and in addition liquefied serum slightly, and after coagulating milk subsequently liquefied the clot. Birge (1905) later isolated the same organism. He was able to show that the coagulation of milk by this form was, in part at least, due to the production of a coagulating enzyme. A similar streptococcus was found by Harrevelt in decaying meat and was named Str.carnis by Chester (I90I).

The first actively liquefying streptococcus described appears to be Str. coli gracilis; and this species may be taken as the type center of the liquefying streptococci. $M$. zymogenes is apparently an extreme form of the same group. A streptococcus of this sort, studied by Gordon (I905), acted on salicin and mannite tho not on raffinose and inulin; and the same fermentative. power was found 
by Houston (1905) associated with liquefaction of gelatin and of the casein in coagulated milk. This type is therefore apparently most closely allied to Str. facalis (Andrewes and Horder). In the absence of more extended studies of fermentative power it is impossible to characterize it with any fullness. It may be provisionally defined as follows:

7. Str. GRACILIS (Escherich, Lehmann and Neumann). Small coccus occurring in chains. Ferments lactose and coagulates milk. May ferment salicin and mannite. Liquefies gelatin, actively.

The various streptococci which peptonize gelatin, more or less actively, may be considered as variants of this type, intermediate between it and some of those characterized by Andrewes and Horder. Under this head come Str. coli (Escherich), Migula, Str. gracilis (Escherich), Migula, Str. septicus (Babes), Migula, Str. urea (Rovsing), Migula, Str. trifoliatus (Rovsing), Migula, Str. rugosus (Rovsing), Migula, Str. vini (Kramer), Migula, $M$. liquidus, Migula, and $M$. decolor, Migula.

Another peculiar form which we have twice found in the course of our own investigations, and which may possibly deserve specific rank, is Streptococcus sanguineus, described by Migula (I900), and distinguished by the formation of a distinct brick-red pigment (intensely blood-red according to Migula) in the stab, - the surface growth being colorless. The fact that old stab cultures of streptococci sometimes showed a reddish-brown color- 
ation was observed by Kurth (I891); but Pasquale (1893) first noted that certain strains possessed this chromogenic power in a very marked degree, forming under anaerobic conditions an almost blood-red growth in two or three days. He found this property, too, to be correlated with the virulence of the cultures when injected into rabbits, the chromogenic strains being always most pathogenic. It is interesting to note that in two other families of bacteria the same peculiar phenomenon of a red pigment under anaerobic conditions has been recorded, (Spirillum rubrum, v. Esmarch, and Bacillus rubellus, Okada). Further comparative studies are needed before this type can be recognized as a distinct species. 


\section{CHAPTER VIII.}

\section{THE GENUS AUROCOCCUS.}

Pyogenic organisms, differing in morphology from the streptococci, were recognized by Ogston (I880), before the advent of the pure culture method, and called by him Staphylococci. Rosenbach (I884) described two varieties of the Staphylococcus pyogenes, which he named var. aureus and var. albus, from the orange and white color of their respective growth masses. Later, a. third type, producing a lemon yellow pigment, was recognized by Passet (1885). The group of the staphylococci generally includes all cocci found on the surface of the body, or in diseased conditions, which do not occur in chains, and which produce a fairly abundant growth on media, whether white, yellow, or orange. Organisms of this general type are normally present in the respiratory and intestinal tract of man, on the other exposed surfaces of the body, and to a considerable depth in the skin. They are the commonest forms associated with suppurative

? processes, causing acne, carbuncle, abscesses, osteomyelitis, endocarditis, and even puerperal infection and general septicæmia. As secondary invaders, they cause serious complications in such diseases as tuberculosis and the exanthemata. 
The higher animals appear to be parasitized by staphylococci closely related to those found in man. The cocci present as secondary invaders in the tuberculous nodules of cattle have been studied by several observers; and the results of earlier workers, with a study of thirty-two new cases, are cited in an admirable paper by Oestern (I904). The author concludes that the organisms are identical with those found in human suppurations. The commonest form in twenty-six cases was a white coccus, positive to the Gram stain, liquefying gelatin and coagulating milk, which we define later as our Albococcus pyogenes. An orange form was found seven times and a yellow micrococcus three times.

It has been held by almost all medical observers that the distinction between the three chromogenic varieties of "the staphylococcus" is an insignificant one. Thus Neisser and Lipstein (1903) hold that the white pyogenic cocci are indistinguishable from the orange forms in distribution or pathogenicity or in any biological properties, save pigment formation. They believe the white cocci arise from orange strains which have lost their chromogenic power through the effect of external conditions. In a recent review, Courmont (I906) will scarcely allow varietal rank to the wite and yellow forms.

The most important evidence of the unity of the staphylococci lies in experiments which have demonstrated the variability of their chromogenic power. Rodet and Courmont (I890) claim to have witnessed, in successive cultures, 


\section{I74 RELATIONSHIPS OF THE COCCACE}

the transformation of a white staphylococcus to an orange one, and back again to a white one; but the possibility of mixed cultures is too great to permit the acceptance of such a statement without strong confirmatory evidence. In no other case, as far as we are aware, has the acquisition of the power of forming orange pigment been recorded. The change from a chromogenic to a non-chromogenic form has, on the other hand, been demonstrated by many observers. Lubinski (1894) noted that the orange staphylococcus lost its chromogenic power under anaerobic conditions, and that the recovery of this property on subsequent transfers to aerobic cultures was sometimes delayed or abolished. After cultivation in an atmosphere of carbon dioxid, three days' growth in air was sufficient to restore the chromogenic power; while after cultivation in hydrogen, aerobic cultures showed, after fifteen days, a notably paler color than those which had not been exposed to unfavorable conditions. After ten transfers under anaerobic conditions the chromogenic power was apparently destroyed, and even after transfers in air the culture could not be distinguished from the white staphylococcus. Lubinski attempted to produce chromogenic power in strains of the white staphylococcus, but without success.

Kolle and Otto (I9O2) note that heating to 85 degrees produces a non-chromogenic strain of the orange staphylococcus, and that the same loss of chromogenic power follows prolonged cultivation on artificial media, or repeated passage through animals. Konradi (I9O4) pointed 
out that, when kept in tap water or distilled water, the orange staphylococcus lost its orange color and again recovered it after animal inoculations. In an earlier communication (Winslow and Rogers, I906) we have shown that exposure to as low a temperature as 50 to 55 degrees may produce a subsequent loss of chromogenic power. Too strong light, or too long cultivation in complete darkness, as well as the presence of chemical poisons, are said to hinder chromogenesis (Neisser and Lipstein, I903).

Differences in pigment production appear also to arise among the cocci as the result of spontaneous variations, - not, as in the cases quoted above, in response to unfavorable environmental conditions. Conn (I900) and Sullivan (I905) note that on a plate, sown from a pure culture, there may develop colonies varying appreciably in shade, from which, by selection of the extremes, quite distinct types can be derived. Neumann (1897) records the sudden appearance of widely different strains, as sectors in old and carefully sealed stab cultures. We have observed both phenomena in our own work, but are inclined to attribute the second to contamination.

Medical bacteriologists, from practical experience, have recognized the pyogenic staphylococci as a fairly well defined natural group; and in view of the unstable character of the pigment-producing power they have generally considered the orange and white forms as only varietal types. Published descriptions were, however, 
inadequate to distinguish the "staphylococci" of the body from the yellow and white cocci found elsewhere in nature. In the systematic compilations of Migula (I900) and Chester (IgOI) parasitic and saprophytic forms were indiscriminately mingled. It was necessary, however, to find some basis for grouping the mass of micrococci to which specific names have been given; and both authors resorted to chromogenesis as a primary basis of classification. The species listed by Migula under the generic name Micrococcus, more than two hundred in number, were arbitrarily divided according to chromogenesis and liquefaction. As a result, the white and orange staphylococci were separated from each other, and associated with forms of widely different origin and nature.

The only attempt at a natural biological arrangement of these organisms, with which we are acquainted, was made by Unna (I900) in a study of the cocci found on the skin in cases of eczema. The primary basis of his classification was the size of the cocci and their behavior during division. Five types of multiplication were recognized, according to the number of cell-divisions which took place before the "hull" or connecting membrane between the cells divided also. Certain cultural and biochemical characters were observed; and Moberg and Unna (I900) finally divided one hundred and forty-five cultures, obtained from seventy-four patients, into twentythree general classes, characterized by the size of the cells and cell masses, the comparative growth at 20 and 37 
degrees, the action of the organisms upon milk and gelatin, and their chromogenic power. The morphological characters upon which this classification was based do not seem to us of great weight. The occurrence of the same coccus in larger or smaller cell groupings, according to varying conditions, is a matter of common observation, and the size of the individual cell is also notably inconstant. The last three of Unna's types are apparently sarcinæ, which occur in larger or smaller growth masses; and the twelve classes under these three types, including fifty-seven cultures, were all alike in failing to acidify milk and liquefy gelatin. The first two growth-types of Unna, micrococci occurring singly or in pairs, included eleven classes, differing in chromogenesis, liquefaction, and lactose fermentation. Of these classes, I, 2, 3, and Ir, including sixteen cultures, acidified milk, liquefied gelatin, and produced a yellowish growth. Class 9 (four cultures) differed in its grayish-white growth; Class 5 (one culture) in its white growth and failure to liquefy. Among the non-acid formers, Class 4 included three cultures of yellow liquefiers, Class 6, two cultures of white liquefiers, Class 7 , a single orange non-liquefying form, Class 8 , five white non-liquefiers, and Class Io, two yellow non-liquefiers.

In this investigation, the fundamental difference between the orange and yellow chromogens was again ignored. The comparative quantitative study of biochemical characters in a considerable series of cultures 
has led us, however, to the belief that, in spite of its instability, the power of pigment production is, under natural conditions, correlated with the most important group differences among the cocci. The facts which pointed to this conclusion have been fully summarized in Chapter IV, and it is unnecessary to repeat them here. It appeared that the orange and white forms were characteristic of the normal or diseased body, while the lemonyellow cocci usually occurred as saprophytes in earth, water, or air. The former generally stained by Gram and produced acid in dextrose and lactose. The latter were, as generally, Gram-negative and, as generally, failed to ferment sugars. The orange and white forms clearly belong to the great division of the Coccaceæ, adapted to life on or in the animal body; the yellow forms belong to the other great division which includes the saprophytic cocci. Furthermore, the orange and white types differed from each other in their action upon gelatin as well as in chromogenesis. Among the liquefying organisms in the two groups the orange chromogens were twice as active as the white forms.

Since several well-marked subtypes could be recognized within each of these two main types, it seemed most convenient, as stated in Chapter IV, to recognize the orange forms (actively liquefying, if at all) and the white forms (with only moderate liquefying power) as distinct generic groups under the names Aurococcus and Albococcus. It is understood of course, as in other cases, that 
these genera are not comparable to those of the higher plants. Intermediate forms exist which connect the two types. As actually found in nature, however, the parasitic cocci group themselves about these two main centers, and as a matter of practical expedience it is convenient to give them names.

These various considerations have led us to the following definition of the genus Aurococcus:

Genus Aurococcus (Winslow and Rogers). Parasites. Cells in groups and short chains, very rarely in packets. Generally stain by Gram. On agar streak good growth, of orange color. Sugars fermented with formation of moderate amount of acid. Gelatin often liquefied very actively. May or may not reduce nitrates.

The members of the genus Aurococcus, as thus defined, are normal inhabitants of the human epithelia and frequent invaders of actively diseased tissues. In virulence, various strains vary widely. Passage through animals generally increases virulence, and the increase is more or less specific for the species of animal used (Neisser and Lipstein, I903). A highly virulent culture will kill a guinea pig of medium size in four to eight days if .I cc. be injected intravenously. Fever and wasting are observed and autopsy shows the presence of numerous local abscesses.

It is from the standpoint of the pathologist that most of the investigations upon these forms have been carried out. These studies have concerned themselves mainly 
with the problems of infection and immunity, and their chief result from the systematic standpoint has been to make clear the existence of virulent and non-virulent types, both of orange and of white staphylococci. The Aurococcus produces both a hemolysin and a substance which acts specifically on the white blood cells, first described by Van de Velde (I894) and called by him a Leucocidin. Neisser and Wechsberg (IgOI) in an elaborate study showed that these were distinct bodies, the strength of one varying independently of the other, and they held the formation of hemolysins and leucocidins to be important aids in differentiating pathogenic staphylococci from other types.

Kolle and Otto (1902) studied the agglutination reactions of Aurococcus and Albococcus and concluded that the serum of immunized guinea pigs would serve to distinguish the true pus-formers from allied types. Klopstock and Bockenheimer (I903) confirmed the general results of Kolle and Otto. They found that a serum prepared by injecting a pathogenic Aurococcus or Albococcus would generally agglutinate other pathogenic forms but not saprophytic ones. Some saprophytic cocci failed to stimulate the production of agglutinins at all. Some, on the other hand, when injected, produced sera which would agglutinate other saprophytic strains but did not affect pathogens. Van Durme (1903) also found that hemolytic power was generally greatest in cultures of albococci and aurococci freshly isolated from pathogenic 
conditions, and was generally absent in cultures from dust and from the normal mouth. Pröscher (I903) records the same distinction, pyogenic cocci in general being agglutinated by the serum of immunized goats, while staphylococci from the air and the surfaces of the normal body were not.

Kutscher and Konrich (I904), also, found that pathogenic staphylococci and those from other sources than disease processes could be distinguished in a general way by their reactions to immune sera, altho individual cultures offered notable exceptions. Pathogenic forms almost always formed hemolysins, while saprophytic forms, as a rule, did not. These results have been confirmed by Veiel (I904), Fraenkel and Baumann (I905), and many other observers. The qualities of virulence are of course among the most unstable of bacterial characters; and immunity reactions of all sorts are quickly modified by the conditions of cultivation. Kayser (I902) and other observers have shown, for example, that cultivation of pyogenic cocci in sugar media leads to a more rapid decrease in virulence and hemolytic power than that which occurs in ordinary broth. It is of interest to note, in this connection, that Loeb found the presence of sugar inimical to the production of peptonizing ferments by the aurococcus (Neisser and Lipstein, 1903). In spite of the instability of these characters there can, however, be little doubt of the fact that pathogenic staphylococci of both the orange and the white types, when freshly isolated, 
differ from similar saprophytic forms, by their power to produce hemolysis, and by their group reactions to the agglutinins present in immune sera.

With the exception of these investigations on immunity reactions, comparative studies of the orange staphylococci are almost wholly lacking. Gordon (I905) examined the fermentative power of two cocci of this type, and found that both liquefied gelatin, coagulated milk, reduced nitrates, and fermented maltose, lactose, glycerin, and mannite. In the next year he described (Gordon, I906) three more cultures which exhibited the same characters, except that one failed to coagulate milk. It is said that "staphylococci" form hydrogen sulphid and ammonia under the proper conditions and reduce various organic dyes (Neisser and Lipstein, I903). All these points are worth comparative study and may serve to distinguish new species of Albococcus and Aurococcus.

The finer relations of this group can only be cleared up by an exhaustive study of a number of characters in a large series of cultures, such as Andrewes and Horder made of the streptococci. In the course of our investigation on the generic types of the Coccaceæ, we examined one hundred and eighty strains belonging to the genus Aurococcus, and the records of these observations make it evident that at least three types occur within the genus. It is much to be desired that this work should be extended. A study of a larger number of cultures, and particularly a study including determinations of serum reactions and fermentative 
power in different carbohydrates, would no doubt lead to the identification of other specific centers.

The largest number of our strains grouped themselves about a center, of which the organism commonly known as the Staphylococcus pyogenes aureus, Aurococcus aureus, according to our terminology, may be taken as a type. This form shows strong acid production and rapid liquefaction of gelatin but fails to reduce nitrates. The Aurococcus of this type produces an acidity of about .005 normal in either dextrose or lactose broth, after fourteen days at 20 degrees, and liquefies gelatin in a tube of $\mathrm{I} \mathrm{cm}$. diameter to a depth of $2.5 \mathrm{~cm}$. in thirty days at 20 degrees (Winslow and Rogers, I906). Of the one hundred and eighty cultures of aurococci studied, ninety-six fell in this group. Some strains were of course weaker, and some stronger, than the normal in acid production and gelatin liquefaction. On the whole, however, the group was a fairly homogeneous one. Eighty-one out of the ninetysix cultures were isolated from the human or animal body, either from pathological lesions or from the normal skin. The Gram stain was generally positive, only one culture showing two successive negative results. Most of the cultures grew equally well at 20 and 37 degrees (eighteen growing better at 37 degrees), but seventy-two of the ninety-six showed better color production at 20 degrees.

This type is evidently the Staphylococcus pyogenes, var. aureus, of Rosenbach, the M. pyogenes of Lehmann and 
Neumann, and the $M$. aureus of Migula. We have given it the name Aurococcus aureus, and its characters may be defined as follows:

I. AUr. AUReus (Rosenbach) Winslow. A parasitic coccus, living normally on the surface of the human or animal body, or in diseased tissues. Occurs singly, or in pairs, or irregular groups, rarely in short chains. Generally stains by Gram. Good to abundant surface growth of orange color. Moderate acid production in dextrose and lactose broth. Nitrates not reduced. Growth good at both 20 degrees and 37 degrees, but pigment production much better at 20 degrees. Gelatin liquefied rapidly.

The following names of yellow cocci listed by Migula and Chester are apparently synonyms of Aur. aureus: M. subflavidus, Migula; M. Beri-Beri, Pekelharing; $M$. Biskra, Heydenreich; M. lobatus, Migula; M. cremoides aureus, Dyar; M. chryseus, Frankland; M. aqueus, Migula; $M$. argenteus, Migula; and $M$. madidus, Migula.

A second group of the Aurococcus cultures, studied by us, was distinguished from the Aur. aureus type by failure to liquefy gelatin, no peptonizing action being observed after fourteen days. Comparison of other biochemical characters showed further differences; and the frequency curves plotted in Fig. III clearly indicate that liquefying and non-liquefying types, in this and other genera, constitute distinct and separate centers of variation. The organisms of the genus Aurococcus, which failed to liquefy gelatin, formed little acid in lactose media and 
produced a distinctly weaker growth on the streak than was characteristic of Aur. aureus. Forty-nine cultures exhibited these general characters. Thirty-four of them were from the animal body; only one, however, was associated with diseased conditions, which is perhaps significant in view of the general weakness of these strains. Fourteen cultures, a large proportion, were from the air. In relation to the Gram stain and in the power of dextrose fermentation these forms resembled Aur. aureus. In lactose broth, on the other hand, they were less active. Thirteen of the forty-nine cultures formed no acid, and twenty-eight more produced less than .005 normal. These forms were notably favored by the body temperature. Eighteen of the forty-nine grew better at 37 degrees than at 20 degrees, while 75 per cent of the Aur. aureus strain grew equally at the two temperatures.

This type is apparently an offshoot from the Aur. aureus which has lost the power of liquefying gelatin and of fermenting lactose, and which grows more feebly on media than the former species. The frequent occurrence of nonliquefying orange cocci seems to warrant the recognition of this type center by a specific name. It appears to be the form described by Cohn under the name $M$. aurantiacus; and M. siccus, Migula, Str. ochroleucus (Prove), Trevisan, and Str. aurantiacus (Bruyning), Chester, are probably synonyms. Cohn's name is, of course, the earliest and should stand for the type, which may be defined as follows: 
2. Aur. aurantiacus (Schröter, Cohn) Winslow. $A$ parasitic coccus, living normally on the surfaces of the human or animal body, or in diseased tissues. Occurs singly, or in pairs, or irregular groups, rarely in short chains. Generally stains by Gram. Meager to good surface growth, of orange color. Acid production moderate in dextrose broth, but slight in lactose broth. Nitrates not reduced. Growth better at 37 degrees, but pigment production better at 20 degrees. Gelatin not liquefied.

The third type, among the aurococci, differs from Aur. aureus by possessing the power of nitrate reduction, and by other tendencies which ally it to a certain extent with the saprophytic Metacoccaceæ. Thirty-five cultures, out of our series of one hundred and eighty, showed the power of reducing nitrates and may be classed with this type. There was an important difference in the end product of the action of various strains. Ten cultures gave positive tests for both nitrites and ammonia in a large proportion of the tubes tested (ten in number for each culture). Ten of the strains showed ammonia but not nitrites, $/$ and fifteen strains showed nitrites but no ammonia. It was thought at first that this difference might be due to the fact that different strains carried out, more or less completely, a reduction, first from nitrates to nitrites and then from nitrites to ammonia. A special study was made of this point, by inoculating a series of tubes with certain selected cultures and examining one tube a day for two weeks, and by inoculating large flasks of 
nitrate medium and withdrawing portions for examination at intervals for three weeks. These experiments showed that in some cultures nitrites appeared first, while both nitrites and ammonia were found on succeeding days. With other cultures nitrites alone were found, and with still others the ammonia test alone was positive, day after day. Apparently some of the cocci produce ammonia in the peptone-nitrate medium without the formation of nitrites. In a very careful study of the action of sewage bacteria upon nitrates, Gage (I905) has shown that the direct reduction of nitrates to ammonia without the intermediate production of nitrites is unquestionably accomplished by certain forms. In other respects the ammonia producers among the aurococci apparently resemble the nitrite producers; and in the absence of further evidence it seems best to include them with the latter. Including all the aurococci which produce either ammonia or nitrites in nitrate media, thirty-five cultures belonged to this type. Twenty of the thirty-five were from the normal or diseased body and eleven from the air. Acid production was about the same as in the Aur. aureus type. The Gram stain, however, was negative in eight strains on two successive tests and negative eleven times in one test out of two. Of the eight Gram-negative cultures, five were from air and one from earth. The proportion of strains which showed better color production at 20 degrees was smaller in this type than in either of the pre- 
ceding ones. Eighteen of the thirty-five cultures showed better chromogenic power at 20 degrees, but fifteen were as good at 37 degrees as at 20 degrees, and two were better at 37 degrees. Five cultures, which we have classed with this type, failed to liquefy gelatin, and it is possible that a fourth type center of nitrate-reducing non-liquefying aurococci should be recognized. We have felt it unwise, however, to create specific names for rare aberrant strains; and until there is evidence that such forms are sufficiently common to constitute a true center of variation it seems best to let them pass as "variants by subtraction" from the main nitrate-reducing type.

The only published description of a nitrate-reducing orange coccus with which we are familiar is that given by Dyar under the name Merismopedia mollis (Dyar, 1895). According to Dyar's description, the organism with which he worked did not change the color of litmus-lactose media, but did coagulate milk and liquefy the clot. It seems possible that there may have been some error in the observation, or in the description, as no other case has been recorded in which a coccus has coagulated milk except by acid production. Dyar's name, with emended characters, may, however, be applied to our third type center and characterized as follows:

3. AUR. Mollis (Dyar) Winslow. A parasitic coccus, living normally on the surfaces of the human or animal body, or in diseased tissues; often found in the air. Occurs singly, or in pairs, or irregular groups, rarely in short 
chains. Reaction to Gram stain variable, more often positive than not. Good to abundant surface growth, of orange color. Acid production moderate in dextrose and lactose broth. Nitrates reduced to ammonia, or nitrites, or both. Growth generally equal at 20 and 37 degrees; pigment production equal, or better at 20 degrees. Gelatin usually liquefied rapidly; rarely, not liquefied.

These three species are the only ones which seem clearly established in the light of present knowledge. The work on immunity reactions, which has previously been reviewed, makes it clear that a group of aurococci exists which differs from the common pyogenic Aur. aureus in lacking hemolytic power, and in failing to agglutinate with immune sera. These differences in pathogenic properties have not so far been correlated with a proper study of biochemical characters. The non-pathogenic forms may correspond with Aur. aurantiacus or with Aur. mollis or with a new type resembling Aur. aureus, except in virulence. Without further study of these points it seems unwise to complicate matters by the introduction of a new specific name, based solely on low hemolytic power and failure to agglutinate. Comparative work on serum reactions and biochemical characters should be fruitful here.

The orange cocci occasionally found in water or in earth, like those which occur even more commonly in air, are apparently stray parasitic forms which have persisted in an unnatural environment, perhaps with the loss of 
certain of their native powers. The occurrence of these forms is rare. Of our one hundred and eighty cultures of orange chromogens, one hundred and thirty-five were from the normal or diseased body, only six from earth, six from water, and thirty-three from air. The infrequency with which orange cocci have been described from sources other than the body makes it clear that there is no important saprophytic source for organisms of this type.

One exception must be noted to the general rule, discussed in Chapter IV, - that the chromogenic cocci of the body are orange pigment formers, and the saprophytic cocci of the earth and water are yellow. It is well recognized that staphylococci producing a lemon yellow pigment (Staphylococcus pyogenes citreus, Passet) are found in appreciable numbers on the normal skin and occasionally penetrate the tissues in disease. Otto (I903) has shown that these yellow forms produce hemolysins and leucocidins and react to agglutinins, just as the pathogenic aurococci and albococci do. Only a careful quantitative study of the acid production, gelatin liquefaction, and chromogenic power in a considerable series of these forms will show whether they are aurococci which have suffered a change in pigment production, or micrococci which have acquired pathogenic powers. In our investigation we have examined thirteen cultures of pathogenic yellow pigment formers, and this small number showed low acid production, a generally negative reaction to the Gram stain, and a 
rather slow liquefaction of gelatin. In the absence of more extended studies we are, therefore, inclined to refer these forms to Micrococcus. It is at any rate clear that they form a minor connecting link between two much larger and more sharply defined groups, - the parasitic aurococci and the saprophytic micrococci. 


\section{CHAPTER IX.}

\section{THE GENUS ALBOCOCCUS.}

IT has been recognized by all students of the bacterial flora of the skin, since Rosenbach, that cocci producing a somewhat abundant white growth are among the commonest organisms found. These forms have generally been classified by dermatologists and pathologists with the orange pigment formers, under the general term "staphylococci." On the other hand, systematic bacteriologists, like Migula and Chester, have grouped the white staphylococci of the skin with white saprophytic forms, and the orange staphylococci with yellow saprophytic forms. All organisms not normally found in chains or packets are included by them in the genus Micrococcus; and this genus is divided into arbitrary groups according to chromogenesis and liquefaction, without reference to habitat.

We have pointed out in Chapter IV the reasons which led us to conclude that the orange and white cocci of the skin should be separated from the saprophytic micrococci of the earth and water. The parasitic organisms show definite group differences, in reaction to the Gram stain, in extent and character of surface growth, in acid production, and, to some extent, in gelatin liquefaction, which 
mark them off as distinct from the saprophytic forms. Furthermore, the white forms differ from the orange ones so distinctly, in abundance of surface growth and in action upon gelatin, as to warrant their separation as a distinct type center, for which we have suggested the name Albococcus.

The orange staphylococci differ from the yellow saprophytic forms in almost every point; and the chromogenic power seems a trustworthy mark of these group differences, since orange pigment formers are very rarely found except on or in the animal body. When they are isolated from water or earth their presence may generally be accounted for by excretal pollution. The white staphylococci, on the other hand, are less easily distinguished from the micrococci, for two reasons. In the first place, the typical Albococcus is somewhat intermediate in characters between Aurococcus and Micrococcus. It resembles Aurococcus in reaction to the Gram stain and in fermentative power; it resembles Micrococcus in abundance of surface growth and slow action on gelatin. In the second place, observation of chromogenesis alone is not sufficient to distinguish true albococci from certain saprophytic forms. The earth and water bacteria belonging to the genus Micrococcus usually produce a yellow pigment; but not infrequently forms will be found in a saprophytic environment which possess the fermentative powers and show the Gram reaction of Micrococcus, but give a whitish or light yellow growth on media. As a rule some tinge of 
yellow may be detected by the method of examination described in Chapter III. To the casual observer, however, the growth of such micrococci will appear white; and these forms can only be distinguished from the albococci by fermentative power and reaction to the Gram stain.

The type center of the albococci has been defined as follows:

Genus Albococcus (Winslow and Rogers). Parasites. Cells in groups and short chains (never in packets). Generally stain by Gram. Growth on agar streak abundant, and porcelain-white in color. Sugars fermented with production of a moderate amount of acid. Gelatin liquefaction and nitrate reduction may or may not occur.

Recent medical literature offers abundant evidence of the ubiquity of Albococcus pyogenes (under the names Staphylococcus pyogenes albus and Staphylococcus epidermidis albus) on the surfaces of the body, and its occasional connection with processes of disease. It may be associated with practically all the pathological conditions characteristic of the Aurococcus, or it may occur fairly deep in the layers of the skin without any evidence of malign influence. Albococcus pyogenes has been isolated from suppurative diseases in many animals, recently, for example, in the hare (Bürgi, I905).

The virulence of the white staphylococci varies widely, and a considerable mass of evidence, summarized in Chapter VIII, shows that among the white, as among the orange forms, pathogenic and non-pathogenic types may be 
distinguished, differing in hemolytic power and in response to agglutinating sera. The pathogenic form is the Staphylococcus pyogenes albus (or Micrococcus pyogenes); the other is probably the one described under the name Staphylococcus epidermidis albus. Until these data with regard to immune reactions are correlated with observations of other biological characters, it is impossible accurately to define the avirulent type.

The most important contribution to the comparative biology of this genus has been made by Gordon, who extended his studies on the fermentative powers of the streptococci to the "staphylococci," and mainly to the white forms. These organisms were found (Gordon, I905) to differ from the streptococci in producing acid somewhat more slowly, in reducing nitrates, in failing to ferment the glucosides, salicin and coniferin, in liquefying gelatin, and in other less sharply marked reactions. Nine tests were selected, as being of most value for the differentiation of the white staphylococci, including liquefaction of gelatin, coagulation of milk, reduction of neutral red, and the fermentation of lactose, maltose, glycerin, and mannite. Forty-one strains were examined by these tests, and the commonest type was found to be the one which liquefied gelatin, reduced nitrates, and fermented maltose and glycerin, but failed in the other five reactions. In the next year Gordon (I906) examined over three hundred strains of staphylococci. All, without exception, showed a positive reaction to the Gram stain, altho seven cultures 
failed to do so on the first trial. In broth, two types of growth appeared:-diffuse turbidity was associated with the microscopic picture of single cocci and pairs; sediment, below a clear liquid, was associated with the occurrence of cells in larger masses. The application of the nine tests mentioned above, with the exception of the peptonization of the milk clot, which was generally negative, showed that one type of albococcus is characteristic of the skin, being predominant on the hand, cheek, scalp, and forearm. It was characterized by turbidity in broth, coagulation of milk, liquefaction of gelatin, change in neutral red, reduction of nitrates, and fermentation of lactose, maltose, and glycerin, but not of mannite. On the scalp, a second type appeared in almost equal abundance, which was negative in all these respects, except in nitrate reduction and in the fact that it formed acid in mannite. On the hand and cheek, a considerable minority belonged to a type fermenting maltose and lactose, but giving negative results in other respects. A few cultures from the air showed somewhat different characters, altho the number studied was insufficient to warrant definite conclusions.

The second of Gordon's types, found on the scalp, is a somewhat puzzling form to classify. From its positive Gram reaction and its apparently common occurrence on the body it should be an Albococcus. On the other hand, its failure to form acid in lactose broth and other sugar media would place it with the micrococci. It is quite possible that pale yellow and whitish micrococci, of 
originally saprophytic origin, may be found at times upon the skin. This is evidently the case with the more distinctly yellow forms grouped under the name Staphylococcus pyogenes citreus, which, as we have pointed out above, are probably allied with the saprophytic micrococci. It is possible, on the other hand, that such forms as those described by Gordon may be simply strains of albococci which have lost their normal powers. They may therefore constitute, either a special type center of albococci with low fermentative powers, or a special type center of micrococci partly adapted to life on the body. It seems wisest to await further evidence as to the quantitative characters and distribution of such forms before giving a name to the type center. The other two of Gordon's types, on the other hand, are clearly albococci, distinguished from each other by their action upon gelatin, nitrates, and glycerin. Type I liquefies gelatin, reduces nitrates, acidifies glycerin, and clots milk; type III does none of these things. Both forms have been recorded by other observers, and both have been observed by us in our series of cultures.

In our own investigations we found twenty-three out of five hundred cocci which could be classed as albococci. All were obtained from the body or from air. All showed a good surface growth of white color. Sixteen were Grampositive on two successive tests, two Gram-negative, and five variable. The average acidity in dextrose broth was .007 normal and in lactose broth .005 normal. In 
these respects the group was homogeneous. Three cultures showed the gelatin liquefaction and nitrate reduction, characteristic of Gordon's first type. Nine failed to liquefy gelatin or reduce nitrates (Gordon's third type). The other eleven liquefied gelatin but failed to reduce nitrates, and therefore corresponded with the commonest described form of the Staphylococcus pyogenes albus. It is curious that this form, which a survey of pathological literature shows to be well-nigh ubiquitous, was isolated by Gordon in only a few cases.

It seems certain from the frequency with which the liquefying, non-reducing albococcus has been reported that it should receive recognition as a distinct specific type. It is the form described by Rosenbach as Staphylococcus pyogenes albus, now more commonly called $M$. pyogenes, and it may be characterized under the name Alb. pyogenes as follows:

I. Alb. pyogenes (Rosenbach) Winslow. A parasitic coccus, living normally on the surfaces of the human or animal body, or in diseased tissues. Occurs singly, or in pairs, or irregular groups. Generally stains by Gram. Good to abundant white surface growth. Moderate acid production in dextrose and lactose broth. Nitrates not reduced. Growth best at 37 degrees. Gelatin liquefied, but somewhat slowly.

Many names have been given to white liquefying cocci; but a study of the published descriptions fails to show any valid characters for their separation. Almost 
all have been isolated from the human or animal body. Acid production and a positive reaction to the Gram stain are recorded, wherever these characters are recorded at. all. The following list includes the "species" tabulated by Migula and Chester which appear to be merely synonyms of Alb. pyogenes.

$M$. acidificans, Migula; $M$. albatus, Kern; M. albescens, Henrici; M. albus, Sternberg; M. alvi, Chester; M. amarificans, Migula; M. coralloides, Zimmermann; M. exiguus, Kern; $M$. faviformis (Flügge), Migula; M. foetidus, Klamann; M. Freudenreichii, Guillebeau; $M$. hemorrhagicus (Klein), Migula; $M$. infuenze, Migula; $M$. lacteus, Henrici; M. lactis, Sternberg; M. lentus, Migula; $M$. liquefaciens (Flügge), Migula; M. mastitis, Chester; $M$. mucilaginosus, Migula; $M$. nitidus, Kern; M. obsconus, Kern; M. ovis, Migula; M. polymyositis, Martinotti; $M$. pseudosarcina, Migula; $M$. pultiformis, Kern; $M$. punctatus, Migula; M. quaternus, Migula; M. radiatus, Flügge; $M$. radiosus (Kern), Migula; $M$. rhenanus, Migula; M. Rheni (Burri), Chester; M.Reesii, Rosenthal; $M$. saccatus, Migula; M. scariosus, Migula; $M$. simplex, Wright; M. subcretaceus, Migula; $M$. subgriseus, Migula; M. sublacteus, Migula; M. subniveus (Henrici), Migula; M. typhoideus, Migula; M. utriculosus, Migula; M. vini (Kramer), Migula; M. xanthogenicus (Friere), Sternberg. As pointed out in Chapter VIII, some of the white cocci of the skin, which come under our general type of Alb. pyogenes, possess distinct pathogenic powers, and 
others do not. The avirulent forms may be of sufficient importance to deserve recognition as a distinct type center. This cannot, however, be considered certain without a careful comparative study of the quantitative biochemical relations of the pathogenic and non-pathogenic forms, since the avirulent white cocci may prove to belong to one or other of the two type centers next to be described.

The second clearly marked type of the albococci differs from Alb. pyogenes in the fact that it reduces nitrates. This was the most abundant form found by Gordon on the hand, cheek, scalp, and forearm. As described by him, it formed enough acid to coagulate milk, formed acid in maltose and glycerin as well as dextrose and lactose, and reduced neutral red. In our own work we found the organism three times; in each case it was isolated from the air. It is apparently the form named by Dyar (1895) $M$. dissimilis. It might well bear this name, as.Dyar's description is the first which specifically includes the property of nitrate reduction. Gordon prefers, however, to identify his type with the Staphylococcus epidermidis albus of Welch. Since Gordon's is the earliest type founded on a comparative study, the name which he adopts from Welch may be accepted as the first to be adequately characterized. It must be modified, however, by the elimination of the trinomial form, in accordance with the general rules of nomenclature. We propose, therefore, the name Alb. epidermidis (Gordon) for this type, characterizing it according to Gordon's description as follows: 
2. Alb. EPIDERmidis (Gordon) Winslow. A parasitic coccus, living normally on the surfaces of the human or animal body. Occurs singly, or in pairs, or irregular groups. Generally stains by Gram. Good to abundant, white surface growth. Moderate acid production in lactose, maltose, and glycerin media. Nitrates and neutral red reduced. Gelatin liquefied, somewhat slowly. . Mannite not fermented.

The third well-marked type of Albococcus is the form which fails to liquefy gelatin. This is the organism described by Gordon as appearing in a large minority among the cocci of the hand and cheek. His cultures exhibited marked weakness in other respects, as well as in their action upon gelatin. They formed insufficient acid to clot milk, did not attack glycerin at all, and failed to reduce neutral red. Nine of our own twenty-three strains fall in this group.

Cohn gave the name $M$. candidus to a white coccus which appeared on potato in his laboratory (Cohn, I872). No other characters were recorded by him; but Migula describes $M$. candidus as a non-liquefying form. The name may, therefore, be taken for the type center of nonliquefying albococci, with the addition of the other characters brought out by recent investigations. In the light of Gordon's work, and our own, the type center may be defined as follows:

3. Alb. Candidus (Cohn) Winslow. A parasitic coccus, living normally on the surfaces of the human or animal 
body. Occurs singly, or in pairs, or irregular groups. Generally stains by Gram. Good to abundant, white surface growth. Moderate acid production in dextrose, lactose, and maltose media, not in glycerin or mannite. Nitrates not reduced. Gelatin not liquefied.

Closely allied to Alb. candidus there appears to be a fourth type of Albococcus, which is probably sufficiently distinct and sufficiently common to deserve a specific name. This is the white, non-liquefying, pathogenic form described by Gaffky (I883) under the name $M$. tetragenus. It is a large coccus, occurring typically in groups of four cells, surrounded by a capsule, and staining by Gram. It is found in human sputum and occasionally develops pathogenic properties, as a secondary invader, or in pyogenic processes. It is extremely pathogenic for white mice, producing an acute septicæmia. On artificial media it grows readily, forming a slimy viscid layer, of a grayish-white color. Its capsule and viscid growth suggest the genus Diplococcus, and its cell grouping is intermediate between that of Diplococcus and that of Albococcus. The abundant growth on artificial media seems to indicate a somewhat closer relation with the latter genus. It is possible that the organism described under the names $M$. botryogenus and $M$. ascoformans, as the cause of botryomycosis in horses (Flügge, I896), is allied to Alb. tetragenus.

It is evident that this organism differs from other albococci mainly in two points: first in the fact that its cells 
occur in the tissues in regular groups of four, surrounded by a capsule, and second in its slimy viscid growth on media. Both characteristics are probably the result of the tendency to zooglea formation. This type, therefore, represents among the white staphylococci the development of the same latent power which shows itself among the diplococci in D. involutus and which is developed to the highest degree in Ascococcus mesenteroides. This type center does not rest on comparative quantitative data as in the case of the earlier ones; but its properties are so striking, and have been recorded by so many observers, as to leave little doubt of its validity. The species may be characterized as follows:

4. Alb. tetragenus (Gaffky) Winslow. A parasitic coccus, living normally on the surfaces of the human or animal body (most commonly in the nose and throat), or penetrating the tissues in disease. Occurs in the body in regular groups of four, surrounded by a capsule. Generally stains by Gram. Good surface growth, of grayish-white color, and viscid consistency. Gelatin not liquefied.

It appears probable that Alb. tetragenus may occasionally produce packet groupings. Lehmann and Neumann (1896) - record this occurrence in hay infusions, and Migula (1900) observed the same appearance in animal tissues. Migula definitely transfers the organism to the genus Sarcina under the name S. tetragena. A form perhaps allied to this was the sarcina isolated by Loewenberg (I899) from a case of ozæna. It produced 
the viscid grayish growth characteristic of Alb. tetragenus, occurred in fours or packets, stained by Gram, and failed to liquefy gelatin or coagulate milk. Its pathogenicity was slight. Two years later Schläfrig (I90I) described a similar organism, and noted that on prolonged cultivation it gradually lost its characteristic type of growth, eventually forming dry porcelain-white colonies.

According to our conception of genera, the genus Sarcina is marked by definite biochemical characters, as well as by morphology. In habitat, Gram reaction, and chromogenesis, as well as in its usual morphology, Alb. tetragenus corresponds with the Paracoccaceæ. On the whole, therefore, it must be retained in the genus Albococcus in spite of the occasional occurrence of packets. Quantitative data with regard to its acid production, which are at present lacking, will throw further light upon this point.

The production of a slimy viscid surface growth is not an unfrequent occurrence among the albococci, and this property alone.is not definitely diagnostic of Alb. tetragenus. M. amarificans, Migula, and M. Freudenreichii, Guillebeau, are forms, otherwise resembling Alb. pyogenes, described as possessing this property in a marked degree. $M$. gelatinogenus, Bräutigam, $M$. gummosus, Happ, $M$. viticulosus, Flügge, and other non-liquefying forms produce growth masses of a notably viscid nature. The importance of this character in relation to morphology and biochemical properties must be worked up, as it has 
been in the genus Diplococcus, before Alb. candidus and $A l b$. tetragenus can be satisfactorily separated.

A large number of names of white, non-liquefying, parasitic cocci appear to be synonyms of one or other of these two types; but whether a given description should be referred to Alb. candidus or Alb. tetragenus is difficult to say, since the descriptions are so generally incomplete in essential points. The following list includes those names which appear to the authors to belong to one or the other of these types: $M$. albocereus, Migula; $M$. asper, Migula; M. canus, Migula; Staph. cereus albus, Passet; $M$. coryza (Hajek), Migula; $M$. fervidosus (Adametz), Migula; M. gelatinogenus, Bräutigam; $M$. gummosus, Happ; $M$. nubilus, Migula; $M$. ovalis, Escherich; $M$. pallens, Henrici; Str. proteus, Chester; M. rugatus (Weichselbaum), Migula; M. salivarius (Biondi), Migula; M. serratus, Migula; M. similis, Dyar; M. tardissimus (Flügge), Migula; $M$. tenuis (Rosenbach), Migula; $M$. tenuissimus (v. Besser), Migula; M. trachomatis, Migula; $M$. viticulosus, Flügge. These forms differ among themselves in abundance of growth and in other minor characteristics. They are alike in the white color of their growth masses and in their failure to liquefy gelatin. They differ from the paler varieties of the genus Micrococcus (to be described in the next chapter) by one or more of the three characteristics of the Paracoccaceæ, parasitic origin, positive Gram reaction, and acid production. 
Whether the organisms which bring about the ammoniacal fermentation of stale urine, $M$. urece and $M$. urece liquefaciens, belong to this genus, and whether they are distinct species or not, can only be determined by comparative study. At present we know neither the other properties of the urine-fermenting forms, nor the extent to which the power of fermenting urine occurs among other well-recognized cocci. Little has been done in recent years in regard to this biochemical property. Comparative studies of various cocci, using perhaps a solution of urea of known composition, rather than urine itself, might well yield results of systematic and theoretical importance. In the absence of such investigations, the question of the systematic significance of the urinefermenting power must be left in abeyance.

Several forms of white cocci belonging to this genus have been characterized by the fact that they produce peculiar star-like or moss-like colonies on gelatin. Among these are $M$.radiatus (of the general type of $A l b$. pyogenes), $M$. cirrhiformis, Migula, $M$. polypus, Migula, and $M$. stellatus, Frankland (allied to Alb. candidus). It is very doubtful, as pointed out in Chapter III, whether differences of this sort are of sufficient systematic importance to warrant their perpetuation in specific names.

Catterina ( 1903 ) has described an organism, apparently belonging to the genus Albococcus, which resembled the last named forms in its production of moss-like colonies, but was actively motile and possessed clearly stainable 
flagella. It was isolated from rabbits which had died of septicæmia, and may perhaps be identified with the motile form isolated by Pitfield (I897) from the blood of dogs and named by him Streptococcus sanguinis-canis. Pitfield's organism was described as forming long chains in certain media, but its white growth makes it improbable that it belongs to the genus Streptococcus as we have defined it. Two other motile cocci have been described which apparently belong to this genus, $M$. tetragenus mobilis ventriculi, Mendoza, and Planococcus casei, Migula. The former resembles Alb. tetragenus in its tendency to occur in tetrads, and in the possession of a capsule. The latter was said to form colonies with toothed edges like Catterina's form.

It is possible that a type center of motile albococci should be recognized. If so, the type form is apparently related to Alb. candidus in other respects. It seems certain, however, that the property of motility is a rare one among the cocci; and it is probable that it is highly inconstant even in those cultures in which it occurs. It has seemed wisest in the light of present knowledge to give specific names only to those types which are apparently so common as to deserve the rank of centers of variation. There seems no reason to depart from this general principle in the present case. Motile cocci of three distinct types have been reported, types which belong in the genera Albococcus, Micrococcus, and Rhodococcus, respectively. Instead of labeling these forms, neither of which has been 
recorded more than two or three times, with specific names, it seems simpler to consider them as variants of the commoner types to which they are respectively allied. Motility, according to this view, is a property which may be developed, under exceptional conditions, in any group of the cocci, altho its manifestation is extremely rare.

Besides these more or less clearly described forms there are a few published descriptions of cocci which their observers failed to cultivate on artificial media, which may be considered as perhaps incompletely characterized albococci. The descriptions of $M$. Beigelii (Rabenhorst), Migula; M. cuniculorum (Schröter), Migula; $M$. decalvans (Thin), Schröter; $M$. dendroporthos, Ludwig; $M$. hyalinus (Ehrenberg), Migula; M. imperatoris, Roze; $M$. nuclei, Roze; and $M$. progrediens, Schröter, are of this indefinite sort. 


\section{CHAPTER X.}

\section{THE GENUS MICROCOCCUS.}

THE name Micrococcus, first used by Hallier, was applied by Cohn to all spherical bacteria (Cohn, I872). The genus has since remained a sort of omnium gatherum, into which all cocci could be put which were not sufficiently characterized for a place in other genera. The strepto- . cocci and sarcinæ gradually came to be recognized as distinguished by their respective chain and packet groupings. The flagella-bearing forms were separated in the genera Planococcus and Planosarcina. Morphological characters did not permit of further progress, and Micrococcus of Migula (I900) and Chester (I9OI) is a kind of residual genus, including all cocci which do not occur in well-marked chains or packets and which do not exhibit flagella.

The classification of the genus Micrococcus as defined by Migula and Chester was naturally a difficult task, since it included such widely different forms as the parasitic diplococci and the saprophytic rhodococci. Migula says, "An arrangement of the individual types of this genus according to their natural relationships is even less possible than in the case of the streptococci. Here, too, we are obliged to rely almost exclusively on uncertain and unreliable biological characteristics" (Migula, I900). 
Migula himself divided his genus Micrococcus (including our Aurococcus, Albococcus, and Rhodococcus) into the following eight groups:

A. Cultivated on gelatin.

I. White growth.

a. Non-liquefying.

$b$. Liquefying.

2. Yellow growth.

a. Non-liquefying.

b. Liquefying.

3. Red growth.

a. Non-liquefying.

b. Liquefying.

4. Blue and violet growth.

B. Not cultivated on gelatin.

Chester arranges the same species in a somewhat more complicated scheme as follows:

A. Without pigment on gelatin or agar.

I. Fail to grow, or grow poorly, on media.

2. Grow on ordinary media.

a. Grow best at 37 degrees and not at 20 degrees C.

b. Grow at 20 degrees C.

* Gelatin liquefied.

** Gelatin not liquefied. 
B. Doubtfully chromogenic. Growth light yellow to yellowish white.

r. Gelatin liquefied.

2. Gelatin not liquefied.

3. No growth on gelatin.

C. Distinctly chromogenic. Form a pigment on gelatin or agar.

I. Pigment yellowish orange.

a. Gelatin liquefied.

b. Gelatin not liquefied.

2. Pigment reddish, pinkish, flesh-colored.

3. Pigment bluish black.

From both these schemes, many of the forms which fail to grow on gelatin may be eliminated, as too imperfectly characterized to be classified at all. Chester's forms which grow feebly on gelatin, or only at 37 degrees, are for the most part parasitic diplococci. The blue and violet forms are apparently short bacilli. No blue-pigment-forming coccus has been recorded by any competent observer in recent years.

A consideration of biochemical characters from the biometrical standpoint shows that this unwieldy genus (including two hundred and twenty-eight species, according to Migula) should be separated into several distinct types. The orange chromogens and a majority of the white forms are distinguished from the yellow and red chromogens by parasitic habit, positive reaction to the Gram stain, and acid production in dextrose and lactose, as well as by pig- 
ment production. Furthermore, the red and yellow forms, while alike in the characters just enumerated, differ from each other in nitrate reduction, and in action upon gelatin, as well as in chromogenesis. These considerations led us to separate the parasitic cocci under the genera Aurococcus and Albococcus and the red forms under the genus Rhodococcus, defining them as described in Chapter IV.

Thus purged of extraneous forms, the genus Micrococcus presents a much clearer and more definite picture. The commonest yellow coccus is recognized by all bacteriologists as a saprophytic type, occurring in water, air, and earth, generally Gram-negative and with slight fermentative powers. Similar forms are occasionally found in water or earth, in which the power of pigment production is so weakened that their growth may appear white. Yellow forms occur, on the other hand, living as parasites on the surface of the body. By far the commonest combination in nature, however, is the yellow saprophytic form; and this marks a well-defined type from which the others deviate. The full definition of the generic type is as follows:

Genus Micrococcus (Hallier, Cohn) Winslow and Rogers. Facultative parasites or saprophytes. Cells in plates or irregular masses (never in long chains or packets). Generally decolorize by Gram. Growth on agar abundant, with formation of yellow pigment. Dextrose broth slightly acid, lactose broth generally neutral. Gelatin frequently liquefied. Nitrates may or may not be reduced. 
It will be noticed that motile forms are not excluded by this definition. The half-dozen cocci which have been described as showing flagella and motility, under certain conditions of cultivation, resemble similar non-motile forms in all other respects. A single character, of a transitory nature, does not seem to warrant the creation of a separate genus. Exaggerated importance has perhaps been attached to this property because of the idea that morphological characters, alone, are of systematic value, and because of the dearth of such characters among the cocci. With the characterization of genera by the correlation of biochemical characters and habitat, the motile forms may be relegated to a more modest rank.

The genus Micrococcus as thus defined is a remarkably simple and homogeneous group. In our own work we have studied one hundred and fourteen strains which apparently belong to it. Thirty-one of these were isolated from the body, thirteen of them from pathological conditions; twenty-eight were from water, twenty-three from earth, and thirty-two were from the air. It is apparent that altho these organisms may occur upon the body, their normal habitat is not confined to it, as in the genera of the Paracoccaceæ. Twenty-five strains were consistently Gram-positive, thirty-eight variable, and fifty-one consistently Gram-negative. The surface growth in one hundred and one cases of the one hundred and fourteen cultures was good to abundant. In dextrose broth nine- 
teen strains showed a reaction between -.00I normal and -.005 normal; sixty-nine a reaction between neutral and .004 normal; twenty-two a reaction between .005 and .009 normal; only four were higher. In lactose broth, forty strains gave an alkalin reaction (between -.00I and -.005 normal); sixty-seven were faintly acid, between neutral and .004 normal; only seven were distinctly acid. Growth and color production were in most cases equally good at 20 degrees and 37 degrees. The pigment production was generally distinctly yellow, falling under the Light Cadmium Yellow, and Medium Cadmium Yellow, in ninety-three cases (see Frontispiece).

The homogeneity of this genus, and the lack of data in regard to fermentative power and immunity reactions, make the distinction of specific types somewhat difficult. Among the Metacoccaceæ we lack the mass of valuable information which has been accumulated by medical and sanitary bacteriologists with regard to the parasitic forms. Such comparative studies as have been made among the diplococci and the streptococci would no doubt reveal the existence of distinct type centers now unknown. It is possible that charactęrs of great systematic importance, here, may be found in properties which have not, as yet, been studied at all; - just as fermentation reactions proved the key to the streptococci.

Meanwhile, a few differential characters are at our disposal, - notably gelatin liquefaction and nitrate reduction. A comparison of these properties in our own 
series of cultures, and a study of the published descriptions in the literature of the group, are sufficient for the recognition of a few of the most obvious types.

The commonest Micrococcus, according to our investigations, is a form which liquefies gelatin but fails to reduce nitrates. Of our one hundred and fourteen strains, fifty-eight were of this type. In habitat, Gram reaction, surface growth, and fermentative power, these cultures conformed closely to the general characters of the genus as previously described. The liquefaction of gelatin was much slower than in Aurococcus aureus but corresponded fairly well with that exhibited by the albococci. Of the fifty-eight cultures, twenty-seven showed a liquefied layer between .6 and r.o centimeter deep, and twenty showed a layer between I.I and I.5 centimeters deep, after two weeks.

The earliest clear description of a liquefying yellow saprophyte appears to be that of Flügge in the I886 edition of Die Mikroorganismen. To this, he attaches the name $M$. flavus liquefaciens, which Migula modifies to $M$. flavus (Flügge). This name may well stand for the first type center of the micrococci. With the additional characters indicated by our own investigation the type may be defined as follows:

I. M. FLAvus (Flügge) Migula. A saprophytic or semiparasitic coccus, found most commonly in earth, water, and air, frequently also on the surfaces of the animal body. Occurs singly, or in pairs, or irregular groups. Generally 
decolorizes by Gram. Good to abundant, yellow surface growth. Reaction in dextrose broth faintly acid, in lactose broth alkalin or faintly acid. Gelatin slowly liquefied. Nitrates not reduced.

A long series of names may be referred, as synonyms, to this species. The principal of them are as follows: $M$. annulatus, Kern; $M$. badius, Lehmann and Neumann; $M$. cerinus, Henrici; $M$. citreus liquefaciens, Unna; $M$. citrinus, Migula; M. confluens, Kern; $M$. conjunctivitidis (Gombert), Migula; $M$. conglomeratus, Flügge; $M$. coronatus, Migula; M. corrugatus (Dyar), Migula; M. cremoides, Zimmermann; M. cupularis, Migula; M. desidens (Flügge), Migula; M. expositionis, Chester; M. flavens, Henrici; $M$. flavescens, Henrici; $M$. flavidus, Henrici; $M$. flavus liquefaciens, Unna; M. galbanatus, Zimmermann; $M$. gigas, Frankland; $M$. luteolus, Henrici; $M$. lutosus, Kern; M. olens, Henrici; M. orbicularis, Ravenel; Staph. pyogenes citreus, Passet; $M$. rugosus, Chester; $M$. subcitreus, Migula; M. subflavus (Bumm), Migula; M. subgranulatus (Freund), Migula; M. subochraceus, Migula; M. tardus (Unna, Tommasoli), Migula; M. Urugua, Chester; $M$. versatilis, Sternberg.

This list includes certain forms, like $M$. coronatus and $M$. rugosus, characterized by differences in the appearance of their colonies which appear to us too slight to warrant specific rank. It includes also the semi-parasitic form, called Staphylococcus pyogenes citreus by Passet, and commonly considered a variety of Aur. aureus. In our 
own series of cultures we have examined twenty-four strains of yellow cocci from the body. In Gram reaction, fermentative power, and rate of gelatin liquefaction, as well as in chromogenesis, they resembled the saprophytic micrococci, and differed from Aur. aureus. It seems more probable, therefore, that this subtype is a form of $M$. flavus, which lives on the surface of the body and may at times acquire pathogenic properties, rather than a form of Aur. aureus, which has lost its Gram reaction and its power to break up dextrose and lactose, changed its chromogenesis and weakened in its ability to act on gelatin.

Another common type of the micrococci differs from M. flavus in its ability to reduce nitrates. This appeared to be the second form in abundance in our investigation, thirty-one of the one hundred and fourteen cultures examined showing the power of attacking nitrates. As in the case of Aur. mollis, discussed in Chapter VIII, some cultures formed ammonia only, some nitrites only, and some both. The first case was the most common. For the present it seems best to include all these forms under a single type, until further study shows how significant the differences may be.

In other respects the biochemical characters of this type correspond to those of $M$. flavus, with a single exception; eight of our thirty-one cultures failed to liquefy gelatin. In the genus Aurococcus a similar phenomenon was noted. Nitrate reducers (Aur. mollis) generally liquefied gelatin, but occasionally cultures were found which 
failed to do so. In the genus Albococcus the nitratereducing form (Alb. epidermidis) is a liquefier. As in the case of Aur. mollis it seems unwise to burden the literature with a name for the rare forms which reduce nitrates but fail to liquefy gelatin. They may be considered as "variants by suppression" from the liquefying type.

For previous information in regard to nitrate reduction one must turn, as usual, to Dyar (1895). He describes a liquefying, nitrate-reducing, yellow coccus from the air and identifies it with the citron-yellow micrococcus of Bumm, called by Flügge $M$. citreus conglomeratus and by Migula $M$. conglomeratus. The descriptions of Flügge and Migula apply equally to $M$. flavus, since the action on nitrates is not referred to. Since Dyar added this character, however, the name he adopted may well be used, modified by dropping the last term of the trinomial and defined with additional characters. Dyar's Merismopedia flava varians is a non-liquefying form, representing the variant by suppression to which reference has just been made. The main type may be defined as follows:

2. M. CITReus (Dyar) Winslow. A saprophytic or semiparasitic coccus, found most commonly in earth, water, and air, frequently also on the surfaces of the animal body. Occurs singly, or in pairs, or irregular groups. Generally decolorizes by Gram. Good to abundant, yellow surface growth. Reaction in dextrose broth faintly acid, in lactose broth alkalin or faintly acid. Gelatin slowly liquefied. Nitrates reduced to nitrites or ammonia. 
The third principal type of the micrococci is the nonliquefying form. In our series of one hundred and fourteen cultures, twenty-five belonged to this type. In two points slight, but possibly significant, differences appear between the non-liquefying micrococci and those previously considered. The Gram stain was more often positive with the non-liquefiers; both slides remained colored in eight cases, in eleven more the result varied, while only six were consistently negative. Again, with these forms the pigment produced had a distinctly orange tinge in certain cases. Nine of the twenty-five cultures showed a color falling in the upper part of the Orange Yellow column of the Frontispiece, and one was in the upper part of the Cadmium Orange. In both respects these strains resemble the genus Aurococcus, and form connecting links with Aur. aurantiacus. Some of our strains might well be considered weakened forms of the latter. Others, on the contrary, were apparently vigorous saprophytic forms with a clear lemon-yellow growth, and the number of cases in which non-liquefying yellow saprophytic cocci have been isolated by other observers makes it probable that the type is a real, and not uncommon, one.

The earliest yellow coccus to be characterized was the Micrococcus luteus of Cohn (I872). It was, of course, not isolated in pure cultures; its growth was observed on potato and in a solution of ammonium acetate. The color and morphology alone were noted, altho it was stated that the pigment was soluble in water. Chester 
(IgoI) describes $M$. luteus as liquefying gelatin, but Frankland and Migula class it as a non-liquefier. Various strains have no doubt been included in laboratory collections under this name. Adopting the more common definition, Cohn's description may be expanded to serve for the type center of non-liquefying micrococci.

3. M. Luteus (Cohn) Migula. A saprophytic or semiparasitic coccus, found most commonly in earth, water, and air, frequently also on the surfaces of the animal body. Occurs singly, or in pairs, or irregular groups. Variable reaction to Gram stain. Good to abundant yellow surface growth. Reaction in dextrose broth faintly acid, in lactose broth alkalin or faintly acid. Gelatin not liquefied. Nitrates not reduced.

A considerable series of names of yellow non-liquefying saprophytic cocci may be considered as synonyms of this species. The following list includes the more important of these: M. butyri (v. Klecki), Migula; M. butyricus (v. Klecki), Migula; $M$. cereus (Passet), Migula; $M$. citreus, Sternberg; $M$. commensalis (Turró), Migula; $M$. cupuliformis, Migula; M. excavatus, Kern; M. flavovirens, Migula; M. gilvus, Losski; M. granulosus, Kern; M. helvolus, Henrici; $M$. Jongii, Chester; M. Lembkei, Migula; M. licheniformis, Kern; M. luridus, Kern; M. ochraceus, Rosenthal; $M$. orbiculatus, Wright; $M$. plumosus, Eisenberg; $M$. resinaceus, Kern; $M$. stellatus, Frankland, $M$. strobiliformis, Migula; M. subgilvus (Henrici), Migula; M. sulfureus, Zimmermann; $M$. tardigradus, Flügge; 
$M$. tenacatis, Chester; $M$. tetragenus-citreus, Vincenzi; $M$. versicolor, Flügge; $M$. vesiculiferus, Migula; $M$. viridisflavescens, Güttmann. Some of the characterizations (M. plumosus, $M$. stellatus, etc.) are based upon supposed differences in colony-morphology; but these seem too uncertain and unimportant for the definition of species.

The three types so far defined, $M$. flavus, $M$. citreus, and $M$. luteus, are the only micrococci with which we have come in contact in our own work. A great many references in the literature make it apparent, however, that a coccus is often found in earth, water, or air which has the other properties of the species last defined, $M$. luteus, but fails to form any appreciable amount of yellow pigment. Its colonies are generally described as porcelain-white. This type was observed as long ago as I886 by two observers; in that year, Flügge called it $M$. candicans, and Bolton, M. aquatilis.

The second of Gordon's staphylococci of the skin, found in considerable numbers on the scalp, apparently corresponds to this type. It resembles Flügge's $M$. candicans in positive Gram stain, and cannot be sharply separated in this respect from $M$. luteus, in which the reaction is sometimes positive. It shows the low fermentative power of the genus Micrococcus, failing to act on lactose, maltose, or glycerin, but producing acid in mannite. It fails to liquefy gelatin and does not meduce nitrates or neutral red.

None of our cultures of Micrococcus, characterized by 
the other properties of the genus - saprophytic origin, negative Gram reaction, low fermentative power, etc. formed an absolutely white growth. On the other hand, there was a slight but distinctly marked type center of paler yellow tint. Six of our twenty-five strains of $M$. luteus formed a pigment which, when matched as described in Chapter III, fell in the upper horizontal line of the color chart (see Frontispiece). Only two fell in the second line and five and seven in the third and fourth, respectively. The numbers are of course too small to draw conclusions from, but the contrast with the other two species, each of which has a single well-marked type center, is suggestive. The distribution of hues for the three species of micrococci is tabulated below.

DISTRIBUTION OF HUES AMONG THE SPECIES OF MICROCOCCI.

Number of Strains of Each Hue.

\begin{tabular}{|c|c|c|c|c|c|c|c|}
\hline Hues $* . . . \ldots \ldots$ & $\mathrm{i}$ & ii & iii & iv & $\mathbf{v}$ & vi & vii \\
\hline M. $\mathrm{fl}$ & 7 & 7 & I5 & I 5 & 5 & 6 & \\
\hline M. citreus. & I & 2 & II & 7 & 4 & 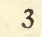 & 3 \\
\hline M. luteus. . & 6 & 2 & 5 & 7 & 2 & 2 & \\
\hline
\end{tabular}

* See Frontispiece.

In view of the small number of strains observed we should not have attached importance to the light center among the non-liquefying forms, except for the evidence from other observers as to the common occurrence of similar organisms in nature. In the light, however, of Gordon's work, and of the numerous descriptions of white non-liquefying saprophytic species, whose names will be listed below, it seems 
permissible to define a type having these characters. Either of the I886 names, M. candicans or M. aquatilis, might be used for this type. Cohn still earlier gave the name $M$. candidus to a coccus which formed white colonies on potato. No other characters were noted, and it is uncertain whether he was dealing with a white Micrococcus - form or with one of the albococci. On the whole, Flügge's name seems preferable, as the first which was described with any fullness.

4. M. Candicans (Flügge). A saprophytic or semiparasitic coccus, found most commonly in earth, water, and air, frequently also on the surfaces. of the animal body. Occurs singly, or in pairs, or irregular groups. Variable reaction to Gram stain. Good to abundant growth, of white color. Fails to form acid in lactose, maltose, and glycerin media, but does acidify mannite. Gelatin not liquefied. Nitrates not reduced.

This type is an intermediate form between Micrococcus and Albococcus. In its white color, and often in its Gram reaction, it resembles the Paracoccaceæ. On the other hand, it fails to attack lactose and maltose, which are broken up by the albococci, and it does acidify mannite, which the albococci fail to ferment. Furthermore, altho it occurs on the body, as all micrococci may, it has been found more commonly under saprophytic conditions.

The following names of white, non-liquefying, saprophytic cocci, which appear to be synonyms of $M$. candicans, testify to the widespread occurrence of this type: $M$. 
achrous, Migula; M. aquatilis, Bolton; M. aquatilis, Vaughan; M. canescens, Migula; $M$. concentricus, Zimmermann; $M$. cretaceus, Henrici; $M$. cyclops, Henrici; M. eburneus, Henrici; M. globosus, Kern; M. grossus, Henrici; M. Hauseri (Rosenthal), Migula; M. humidus, Migula; M. inconspicuus, Henrici; M. Iris, Henrici; $M$. lactis, Chester; $M$. nacreaceus, Migula; $M$. nivalis, Chester; $M$. niveus, Henrici; $M$. odoratus, Henrici; $M$. odorus, Henrici; M. pannosus, Kern; M. parvus, Migula; $M$. roscidus, Migula; $M$. rosettaceus, Zimmermann; $M$. sordidus, Schröter; $M$. subcanus, Migula; M. succulentus, Henrici; M. tardus (Unna, Tommasoli), Migula; M. tetras, Henrici; $M$. tuberculosus, Migula; $M$. vesica, Heim; $M$. zonatus, Henrici.

As was the case in the genus Albococcus, motile forms of micrococci have been described by a few observers. The only one in which the phenomenon is well authenticated is the Micrococcus citreus agilis, described by Menge. In this case the motion of the organism was apparently a true movement of translation, and a single flagellum was demonstrated by Löffler's stain. In other respects the organism resembled $M$. luteus, being a yellow, non-liquefying form. Three other yellow cocci, M. ochroleucus, Prove, M. nasalis, Hack, and Diplococcus luteus, have been described as motile; but in neither case is the description at all convincing.

Reference may finally be made to certain organisms, described as micrococci before the advent of the pure- 
culture method, and still erroneously included in the genus by later observers. Such are $M$. prodigiosus, $M$. chlorinus, $M$. cyaneus, and $M$. violaceus of Cohn (1872), $M$. phosphoreus, Cohn, and $M$. fuscus, Eisenberg. Short bacilli corresponding to all these forms are now well known, while no cocci have been isolated in recent years which form red, green, blue, or brown pigments, or which produce phosphorescence (except the peculiar chromogenic streptococcus that has been discussed in Chapter VII). It is most probable that short rods were mistaken for cocci in the original observations; or the macroscopic characters may have been due to contaminating bacilli in a culture originally made up of cocci. 


\section{CHAPTER XI.}

\section{THE GENUS SARCINA.}

A PECUliar microscopic plant, made up of colorless spherical cells, arranged in packet groups of eight, was described by Goodsir in 1842 (Goodsir, 1842) and named by him Sarcina ventriculi. The generic name Sarcina has since been applied to all cocci which show this cell grouping. Migula (1900) defines the genus by the presence of spherical cells, which divide in three planes at right angles to each other, non-motile and lacking spores.

The genus Sarcina, as thus defined, is much more homogeneous than Migula's genus Micrococcus: it includes no such,parasitic forms as those which we have separated from Micrococcus in our genera Diplococcus, Aurococcus, and Albococcus. Five red pigment formers, however, are classed by Migula in the genus Sarcina. Cocci of the latter type, growing in packets and producing red pigment, are frequently found in nature. In their biological characters these forms correspond closely to the red pigment formers which do not occur in packets, rather than to the yellow sarcinæ. In their almost invariable negative reaction to the Gram stain, in their marked and peculiar action on nitrates (forming in a large proportion of cases nitrites, but not ammonia), and in their rare and slight action 
upon gelatin, the red chromogens, whether they occur in packets or not, form a distinct group of their own. We have, therefore, separated the red packet formers from the genus Sarcina and have referred them to the genus Rhodococcus.

With the elimination of these organisms, the genus Sarcina includes a series of saprophytic cocci producing a vigorous yellow (or whitish) growth on agar and exerting only a slight action on carbohydrate media. In our own series of cultures we have studied one hundred and thirty-seven strains of this general type. They form a compact group and one strikingly parallel to that of the micrococci. How close this parallelism is, has been pointed out in Chapter IV. It need only be recalled here that, in relation to the Gram stain, in action on dextrose and lactose broth, in the effect of temperature on chromogenesis, in the shade of color produced, and in gelatin liquefaction, the quantitative characters of the two genera were the same. The surface growth of the sarcinæ was heavier than in the case of the micrococci, as might naturally be expected. The growth of the sarcinæ was also better at 20 degrees in a larger proportion of cases. Their liquefying action was a little stronger. With these exceptions, and the occurrence of the packet groupings, the two series are alike.

It will be seen in succeeding pages that the specific types included in the two genera are identical, both in characters and in numerical frequency. In view of all these evidences of likeness between the packet-forming and non-packet- 
forming organisms, it seems to the authors likely that the property of the sarcina-grouping is of secondary importance. The name Sarcina is, however, firmly intrenched in the literature and usage of bacteriology. We have felt it wise, therefore, to retain the old genus, pending a consensus of opinion as to its identity with Micrococcus. We have, of course, emended the standard definition of the genus by adding those biochemical characters which we have observed to be associated with the sarcinæ (other than the red pigment formers). With the exception of packet formation, the definition is the same as that of the genus Micrococcus.

Genus Sarcina (Goodsir) Winslow and Rogers. Facultative parasites or saprophytes. Division occurs under favorable conditions in three planes, producing regular packets. Generally decolorize by Gram. Growth on agar abundant with formation of yellow pigment. Dextrose broth slightly acid, lactose broth generally neutral. Gelatin frequently liquefied. Nitrates may or may not be reduced.

Of our one hundred and thirty-seven strains of this generic type, thirty-three came from the body (eighteen of them from diseased conditions), forty-five from water, thirty-one from earth, and twenty-eight from the air. Thirty-two were Gram-positive on each of two trials, sixtythree Gram-negative, and forty-two variable. Fortyfive produced a very heavy surface growth, forty-six an abundant surface growth, forty-three a good surface 
growth, and three only a meager one. In dextrose broth twenty-four strains gave an alkalin reaction, eighty-five produced an acidity below .004 normal, twenty an acidity between .005 and .009 , and eight a higher acidity. In lactose broth forty-one strains formed no acid, eighty-four an acidity under .004 normal, and nine an acidity between .005 and .009, while three were over .oro. Fortythree cultures grew better at 20 degrees than at 37 degrees, and only twelve, better at 37 degrees than at 20 degrees. Forty-nine showed better color production at 20 degrees, and in eighty-two cases chromogenesis was equal at both temperatures. In forty-four cases the color formed could be matched under the Light Cadmium Yellow column of the Frontispiece, in sixty-six cases under Medium Cadmium Yellow, and in twenty-one cases under Orange Yellow. Forty-seven strains failed to liquefy gelatin. Of the remainder, thirteen produced a liquefaction less than .5 centimeter deep in two weeks, twenty-five a liquefaction .6 to r.o centimeter deep, twenty-three a liquefaction I.I to $\mathrm{I} .5$ centimeters deep, twenty a liquefaction I.6 to 2.0 centimeters deep, and eight a liquefaction over 2. I centimeters.

In addition to these characters, it may be noted that Gordon (I906) records the failure of all sarcinæ examined by him to ferment either lactose, maltose, glycerin, or mannite. It is probable, therefore, that the fermentative powers of the group are low in other carbohydrate media, as in dextrose and lactose. 
Migula includes fifty-five species in his genus Sarcina. Fifteen are white forms, liquefying and non-liquefying; thirty are yellow forms, liquefying and non-liquefying; two are brownish forms, five are red forms, and three have not been cultivated on gelatin. Most of these species were described by three authors, Gruber (1897) and Lindner (1888), who studied the disease fermentations in breweries, and Henrici ( 1897 ), who isolated his forms from cheese.

A great deal has been written by various observers about the disease fermentations in beer, produced by sarcinæ. Discussion in recent years has centered about the organisms called Pediococcus damnosus and Pediococcus perniciosus by Claussen (1903). Both these forms produce unpleasant odors and tastes in beer, and both exhibit a special resistance against the antiseptic action of ammonium fluorid. As far as we are aware, no adequate comparative study of these organisms has been made, so that it is not clear whether, or not, they differ from the sarcinæ isolated in other environments.

In this genus, Lehmann and Neumann, and other German systematists, lay particular stress on the fine or coarse granulation of the gelatin colonies. This is a character closely related to the type of packet formation, sarcinæ growing in large packets forming a coarsely granular growth. Alone, and uncorrelated with physiological properties or distribution, it does not seem to us to warrant specific rank. Peculiarities in size of cells are 
noted also in many cases; but among the sarcinæ such observations are dubious, since it is easy to mistake small packets for large single cells. In our series and from a study of the literature, we have found only two characters which seem to vary with sufficient sharpness to mark specific types. These are the same characters which appeared significant among the micrococci, - gelatin liquefaction and nitrate reduction.

As in the genus Micrococcus, the commonest type among our strains of sarcinæ was distinguished by liquefaction of gelatin and by failure to reduce nitrates. Sixty-five of our one hundred and thirty-seven cultures fell in this group. It is unnecessary to discuss in detail the distribution of the members of this type in their quantitative reaction to the various tests, since it corresponded closely to that for the genus as a whole, which has just been stated. The general group of yellow, liquefying sarcinæ has long been a familiar one. Two names were given to organisms of this type in $1887, S$. flava by De Bary and $S$. liquefaciens by Frankland. The former name may be adopted for the type as more extensively defined. It is an interesting coincidence, in view of the parallelism between this species and the yellow, liquefying micrococcus, $M$. flavus, that the corresponding name may be used.

I. S. FlaVa (De Bary). A saprophytic or semi-parasitic coccus, found most commonly in earth, water, and air, also on the surfaces of the animal body. Occurs under favorable conditions in packets. Generally decolorizes by Gram. 
Good to very heavy surface growth, of yellow color. Reaction in dextrose broth faintly acid, in lactose broth alkalin or faintly acid. Gelatin slowly liquefied. Nitrates not reduced.

The following names of yellow, liquefying sarcinæ appear to be synonyms of S. flava: S. baccatus, Migula; S. bicolor, Kern; S. canescens, Stubenrath; S. equi, Stubenrath; S. flavescens, Henrici; S. gigantea, Kern; $S$. lactis, Chester; S. Lembkei, Migula; S. liquefaciens, Frankland; S.livido-lutescens, Stubenrath; S. mirabilis, Kern; S. olens, Henrici; S. radiata, Kern; S. subflava, Ravenel; S. superba, Henrici; S. variabilis, Stubenrath.

It is somewhat interesting to note that none of Lindner's and Gruber's brewery sarcinæ fall in this group. Henrici's cultures were from cheese, and Stubenrath's and Kern's from the stomachs and intestines of mammals and birds.

Besides these typical forms there are a few descriptions in the literature, of similar organisms which, in the light of present knowledge, are harder to classify. The most important of these is the orange sarcina described by Flügge (I886) under the name $S$. aurantiaca. S. aurescens (Henrici), Gruber, and S. aurea, Macé, apparently represent the same form. In our series of five hundred cultures we found one hundred and forty-seven orange micrococci, one hundred and thirty-seven yellow sarcinæ, and only eleven orange forms which showed the packet grouping. The latter combination appears, therefore, to be a rare one. In other biochemical characters our 
eleven strains corresponded with Aurococcus rather than with Sarcina. They were generally Gram-positive, acidforming, actively liquefying forms. We are, therefore, inclined to consider orange packet formers as exceptional aurococci, which grow in packets, rather than as members of the genus Sarcina, which have acquired the biochemical characters of the parasitic genus. $\checkmark$ Other aberrant forms allied to this type are $S$. casei and S. butyrica, both isolated by Migula from cheese. These organisms are characterized by slimy growth masses and by the production of a strong acid reaction in milk. In the absence of further evidence they may be considered as exceptional variants of $S$. flava which have acquired certain fermentative powers.

The second important type among the sarcinæ differs from the first by possessing the power of reducing nitrates. It corresponds in general, therefore, to $M$. citreus. Thirtyfour of our one hundred and thirty-seven strains belong to this type. As with Aur. mollis and M. citreus, some cultures form ammonia, and some, nitrites, while a few show both. All are for the present included under a single type. In regard to their action on gelatin, too, these organisms resemble Aur. mollis and $M$. citreus. Most of them liquefy quite actively, but a few strains (nine of our thirty-four) fail to produce liquefaction. As in the previous cases, we have felt it best to recognize the more numerous liquefying form as a distinct type center, and to leave the question of the existence of another type center, of non-liquefying 
nitrate reducers, in abeyance. If further investigation shows that the latter form is a common one in nature it can then be given a specific name.

The type of the nitrate-reducing sarcinæ does not appear to have been hitherto recognized in the literature. The German systematists rarely observe the nitrate reaction, and Dyar lists only two sarcinæ, neither of them reducers. We therefore suggest for this type the name Sarcina citrea, which emphasizes the parallelism between this form and the corresponding Micrococcus citreus.

2. S. CITREA. (n. sp.) A saprophytic or semi-parasitic coccus, found most commonly in earth, water, and air, frequently also on the surfaces of the animal body. Occurs under favorable conditions in packets. Generally decolorizes by Gram. Good to very heavy surface growth, of yellow color. Reaction in dextrose broth faintly acid, in lactose broth alkalin or faintly acid. Gelatin slowly liquefied. Nitrates reduced to nitrites, or ammonia.

The third important type of the sarcinæ is distinguished by its failure to liquefy gelatin or to reduce nitrates. Thirty-eight of our one hundred and thirty-seven strains fall under this head. The only other differences, of possible importance, between this type and $S$. flava appear in surface growth and in acid formation. In surface growth the non-liquefying forms are somewhat less vigorous than the liquefiers; and the former group includes less actively fermenting strains than the latter. Of our thirty-eight cultures only two produced an acidity over .004 normal 
in dextrose broth, and none exceeded this limit in lactose broth.

The non-liquefying Sarcina has been frequently recorded in the literature. The first clear description of the type was apparently that of Schröter (1886), to which he gave the name of S. lutea; and this name properly stands for the type center, which may be defined as follows:

3. S. LutEA (Schröter). A saprophytic or semiparasitic coccus, found most commonly in earth, water, and air, frequently also on the surfaces of the animal body. Occurs under favorable conditions in packets. Generally decolorizes by Gram. Good to abundant, yellow surface growth. Reaction in dextrose broth faintly acid, in lactose broth alkalin or faintly acid. Gelatin not liquefied. Nitrates not reduced.

Nine "species" isolated from meal and dough by Gruber (1897), and S. sulfurea, found by Henrici (1897) in cheese, appear to be synonyms of S. lutea. Gruber's names are as follows; S. citrina, S. intermedia, S. livida, S. luteola, S. marginata, S. meliflava, S. striata, S. velutina, and $S$. vermiformis.

S. acidificans, Migula, differs from $S$. lutea, according to the author's description, in its power of coagulating milk and later peptonizing the clot. It corresponds in general, therefore, to Migula's other acid-forming sarcinæ, $S$. casei and $S$. butyrica. This may be regarded as an exceptional variant of $S$. lutea if its characters are correctly described. Acid-forming sarcinæ are certainly not common. 
Another interesting Sarcina, possibly related to $S$. lutea, is the form originally isolated by Goodsir in 1842 and named by him $S$. ventriculi. It was described as a non-liquefying sarcina, isolated from the stomach in cases of hyperacidity of the gastric juice. More recent investigations suggest that there is nothing specific in the relation of this organism to the pathological condition in question (Flügge, I896). S. ventriculi was distinguished from the type of $S$. lutea by the production of an orange, instead of a yellow, pigment. It corresponds, therefore, to the non-liquefying $S$. aurantiaca. In discussing the latter form we have pointed out that what little is known of orange packet-forming cocci suggests that they are more closely related to Aurococcus than to Sarcina. Whether Goodsir's form was a packet-forming Aurococcus or an orange Sarcina can only be decided from a study of similar forms which the future may bring to notice.

In the fourth type of the genus Micrococcus the pigment production, characteristic of the genus as a whole, was so modified that the growth masses of the organism appeared white. Among the published descriptions of sarcinæ a considerable number of species are characterized in the same manner. $S$. alba, Zimmermann; $S$. albida, Gruber; S. alutacea, Gruber; S. candida, Lindner; S. devorans, Kern; S. incana, Gruber, are white liquefiers: S. lactea, Gruber; S. minuta, De Bary; S. nivea, Henrici; S. pulchra, Henrici; S. pulmonum, Virchow; S. tetragena (Gaffky), Migula; S. variegata, Pansini; S. vermicularis, 
Gruber; S. Welckeri, Rossmann, are non-liquefying forms. It is possible that a white type center of sarcinæ should be recognized, corresponding to $M$. candicans, in the other genus. The evidence from the literature is less conclusive, however, in this case. Among our own cultures no type center of paler hue was evident, as shown in the table below.

DISTRIBUTION OF HUES AMONG THE SARCINÆ.

Number of Strains of Each Hue.

\begin{tabular}{|c|c|c|c|c|c|c|c|}
\hline 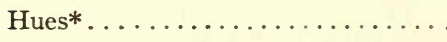 & i & ii & iii & iv & $\mathbf{v}$ & vi & vii \\
\hline S. flav & 4 & I 4 & I9 & I6 & 6 & 3 & 3 \\
\hline S. citrea. & I & 6 & 9 & 7 & 4 & 2 & 5 \\
\hline S. lutea. & 3 & 5 & 8 & I7 & 3 & 2 & o \\
\hline
\end{tabular}

* See Frontispiece.

On the whole it seems best for the present to consider the light-colored sarcinæ as merely variants from the typical forms, - of insufficient importance to warrant the creation of a special type center.

Reference must also be made to several sarcinæ characterized by definite and peculiar properties, but apparently of rare and exceptional occurrence. Gruber (I902), for example, describes an interesting organism, apparently belonging to the genus Sarcina, producing a thick, slimy growth in sugar solutions. This property appears to be latent all through the family of the Coccaceæ, appearing as we have seen in the genus Streptococcus and culminating in the genus Ascococcus. The name given by Gruber (Coccus lactis viscosi) violates every principle of nomen- 


\section{RELATIONSHIPS OF THE COCCACEÆ}

clature. Beyerinck (1905) has recently described an anaerobic, acid-forming sarcina isolated from garden earth. If his observations are correct, this organism offers an interesting example of the secondary acquisition of the anaerobic power in a strongly aerobic group.

The organism described by Gruber as $S$. gasoformans for a time exhibited the power of gas formation, and later lost it. This phenomenon may perhaps be attributed to contaminated cultures. Such fermentative power has not, as far as we are aware, been recorded in any other case. There remain to be considered the two sarcinæ $S$. fusca, Gruber, and S. cervina, Stubenrath, which are described as producing a brownish growth, and three uncultivated forms, S. intestinalis, Zopf, S. maxima, Lindner, and $S$. paludosa, Schröter. The brown pigment formers may be slightly modified forms of the yellow sarcinæ or they may belong to the genus Rhodococcus. It is impossible to determine their relation without the study of new material, which may come to light in the future. 


\section{CHAPTER XII.}

\section{THE GENUS RHODOCOCCUS.}

THE cocci which produce a reddish or pinkish pigment have been classed by systematic bacteriologists in the genera Micrococcus and Sarcina, according to the form of cell grouping observed. They form the ninth group of Chester's Micrococcus (aerobic and facultative anaerobic, - distinctly chromogenic, - pigment reddish, pinkish, flesh-colored) and the fifth group of his Sarcina (red growth on gelatin and agar). In Migula's scheme the fourth main group of the micrococci and sarcinæ cultivated on gelatin includes, in each case, the red forms.

Quantitative study of the biological properties of the red chromogenic cocci in our own series furnished strong evidence of their relation to each other, and of their marked difference from all other members of the family. We : found only twenty-five of these organisms; but their group characters were so definite and constant that even this small number seemed to warrant the creation of the genus Rhodococcus.

First, all our red cocci, but one, were from saprophytic sources, eight from water, seven from earth, and nine from air. Among the yellow micrococci and the sarcinæ about a quarter of the cultures were of parasitic origin. 
Second, only two of the twenty-five strains showed a positive Gram reaction on both trials; seven were variable and sixteen consistently negative. This is a much lower proportion of Gram stains than that found in any other group. Third, the reduction of nitrates was much more common among the red cocci than in other genera; and the nature of the reduction was characteristic. Fifteen of our twenty-five strains reduced nitrates, and in every case nitrites were formed but no ammonia. Fourth, liquefaction of gelatin was rare among the red cocci, and the action was very slow when exerted at all. Only three of our twenty-five strains acted on gelatin in two weeks, and the average depth of liquefaction for these three was only $\cdot 7$ centimeter.

In these four characters the red chromogens differ from all groups previously considered. In a uniform abundant growth, generally equal at 20 degrees and 37 degrees, and in low fermentative power, they resemble the micrococci and sarcinæ. The average reaction of our twenty-five strains was slightly acid in dextrose broth (.003 normal) and neutral in lactose broth. Only four strains formed over .004 normal acid in dextrose broth and only two over .oor normal acid in lactose broth. Finally, in the relation of chromogenesis to temperature the red cocci resemble the aurococci rather than the saprophytic forms. Twenty-one of the twenty-five strains showed better pigment production at 20 degrees than at 37 degrees. 
This definite and peculiar combination of characters clearly marks the red cocci as a natural group of generic importance. Its habitat and negative Gram reaction, low liquefying power, and slow but abundant growth on artificial media seem to place this group at the extreme saprophytic end of the series of the Coccaceæ. Red cocci may of course be occasionally found on the skin, like any other forms common in air or earth, but their occurrence there is apparently exceptional and accidental.

Our twenty-five cultures included ten packet-formers, and fifteen in which the sarcina-grouping was not observed. In all other respects the packet-formers and the non-packetformers were alike. Both series showed the same habitats and Gram reactions, the same chromogenesis, the same peculiar relation to gelatin and nitrates. This is a striking example of the fact that differences in cell-grouping are not correlated with differences in general biochemical characters. It seems clear that the red sarcinæ are related to the similar forms which show no packets, more closely than to the yellow sarcinæ, - which exhibit such distinct group differences in four or five fundamental physiological properties. Logical adherence to the principle followed in dealing with the yellow micrococci and sarcinæ, which are similarly related to each other, suggested the formation of two new genera, - one for the packet-formers and one for the non-packet-formers. It was, however, largely from deference to custom, which must be respected in all matters of terminology, that the genera Micrococcus and Sarcina 
were recognized as distinct. In making a fresh start with the red chromogens we have felt disinclined to form genera, or even species, on the single character of cell grouping. We have preferred to include all cocci possessing the biological powers characteristic of the red chromogens in a single genus under the name Rhodococcus. If further study indicates that the property of packet formation is more important than now appears, it will be easy to form a new genus of red sarcinæ. It is always simpler to add new genera than to eliminate old ones.

We have therefore defined the genus Rhodococcus as follows:

Genus RHodococcus (Winslow and Rogers). Saprophytes. Cells in groups or regular packets. Generally decolorize by Gram. Growth on agar abundant, with formation of red pigment. Dextrose broth slightly acid, lactose broth neutral. Gelatin rarely liquefied. Nitrates generally reduced to nitrites, but not to ammonia.

For defining species in the genus Rhodococcus the same characters are available which have proved significant in other genera, - action upon nitrates and gelatin. The property of packet formation, if not of generic importance, should perhaps deserve specific value. We are not convinced, however, from our own observations that there are really distinct type centers, showing greater and less tendency to the packet grouping. It may well be that all of the red cocci (perhaps all of the Metacoccaceæ) form-packets under certain conditions. Only a com- 
parative study, carried out by some method which will measure this tendency with approximately quantitative results, will form a sure basis for specific types. Such data are at hand, altho on a small scale, in regard to nitrate reduction and gelatin liquefaction, but not for packet formation. It seems best, therefore, for the present to include packet-forming red cocci with those in which the property has not been observed. Packet-forming species may be separated later, if it seems best to do so.

The specific importance of gelatin liquefaction in this group seems also open to serious doubt. Our own observations of the action of these forms on gelatin extended over a period of thirty days. In that time twenty-two strains failed to show any action, two liquefied to a depth of .4 centimeter and one to a depth of $\mathrm{x} .2$ centimeters. The last three forms certainly do not furnish sufficient basis for a distinct species. Red, liquefying cocci have been described by many observers. In almost every case, however, it is noted that the action upon gelatin is extremely slow. Thus Migula (I900) says of $M$. roseus that liquefaction occurs only after a long time and then to a very slight extent; of $M$. rubens, that distinct liquefaction does not occur even after several weeks; of $M$. persicus, that after fourteen days the gelatin begins to soften; of $M$. cumulatus, that gelatin begins to liquefy after fourteen days, etc. Dyar (I895) found that $M$. fragilis began to liquefy slowly after six days, and Sarcina rosea often not for thirty or forty days. It is clear that the rhodococci, when they liquefy at all, do 
so only slowly and feebly. There is no evidence for a distinct type center of actively liquefying forms, separated on the Curve of Frequency from another center of non-liquefiers, such as occurs in Aurococcus, Albococcus, Micrococcus, and Sarcina. It seems best, therefore, not to make a separate species for the slow liquefiers. The most probable conclusion, in the light of present knowledge, is that the red chromogens, in relation to gelatin, form a single group, characterized by entire failure to liquefy or by very slow and feeble action.

There remains only the property of nitrate reduction as a means for differentiating species among the rhodococci. Here our series, altho small, seems to indicate the existence of two distinct types, one forming nitrites in nitrate solution, the other failing to do so. The commonest type according to our somewhat limited observations is a form which reduces nitrates in the manner peculiar to the genus. Of our twenty-five strains fifteen fall under this head. In other respects they correspond to the general type of the genus as previously described. Five are packet-formers, and three, slow liquefiers.

The only published description of a strongly reducing, red coccus which has come to our attention is that of the organism which Dyar identified with Diplococcus roseus of Bumm. Dyar calls it Merismopedia rosea (Bumm). This form was really first named by Flügge (I886) as $M$. roseus, a year after Bumm described it as the "rose colored diplococcus"; but it was Dyar in I895 
who noted the property of nitrate reduction. We may use Flügge's name, with credit to Dyar's emendation, for the first type of Rhodococcus.

I. RHodococcus Roseus (Flügge, Dyar) Winslow. $A$ saprophytic coccus, found most commonly in earth, water, and air. Occurs in pairs, groups, or packets. Generally decolorizes by Gram. Abundant, red surface growth. Reaction in dextrose broth faintly acid, in lactose broth alkalin or faintly acid. Gelatin either not liquefied or liquefied very slowly. Nitrates reduced to nitrites, but not to ammonia.

The second type among the rhodococci is distinguished by failure to attack nitrates. Ten of our strains fell under this head. Five of the ten showed packet formation, and none liquefied gelatin within two weeks. In other respects these strains showed the characters noted above as typical for the genus. The non-nitrate-reducing type may perhaps best bear the name given by Cohn (I875) to a coccus which formed a rust-red growth on horse dung in the laboratory. The type may be defined as follows:

2. Rhodococcus fulvus (Cohn) Winslow. A saprophytic coccus, found most commonly in earth, water, and air. Occurs in pairs, groups, or packets. Generally decolorizes by Gram. Abundant, red surface growth. Reaction in dextrose broth faintly acid, in lactose broth alkalin or faintly acid. Gelatin either not liquefied or liquefied very slowly. Nitrates not reduced.

To which of these types the published descriptions of red cocci refer, it is generally impossible to say, since data 
as to nitrate reduction are almost universally lacking. Of the forms examined by Dyar, $M$. cinnabareus (Flügge) reduced only slightly in twenty-eight days, Merismopedia fragilis failed to reduce, and $M$. roseus sometimes reduced quite actively. The following names are certainly to be referred to the genus Rhodococcus, but whether to $R$. roseus or $R$. fulvus is uncertain:

A. Forms in which neither packet formation nor liquefaction is recorded. $M$. bicolor, Kern; M. carneus, Zimmermann; $M$. cerasinus (Heim), Migula; M. cinnabareus, Flügge; M. cinnabarinus, Zimmermann; M. coccineus, Migula; M.Dantecii, Chester; M.Kefersteinii, Chester; $M$. lactericeus; Freund; M. rhodochrous, Zopf; M. rubellus, Migula; $M$. rubescens, Migula; $M$. sarcinoides, Migula.

B. Forms in which slow liquefaction has been recorded but packet formation has not. M. carnicolor, Frankland; $M$. coralinus, Catani; $M$. cumulatus, Kern; $M$. fragilis (Dyar), Migula; $M$. persicus, Kern; M. rosaceus, Frankland; $M$. roseo-persicinus, Migula; M. rubens, Migula; $M$. rubiginosus, Kern; 'M. subcarneus (Kern), Migula; $M$. sublilacinus, Migula; M. subroseus, Migula.

C. Forms in which packet formation and slow gelatin liquefaction are recorded. S. erythromyxa (Overbeck), Chester; S. rosacea (Lindner), Migula; S. rosea, Lindner; S. rubra, Menge.

D. Forms in which packets have been observed but not liquefaction of gelatin. S. carnea, Gruber; S. incarnata, Gruber; S. persicina, Gruber. 
Minor differences in size and in colony morphology are of course recorded for many of these forms. In several cases a positive reaction to the Gram stain is noted (M. carneus, $M$. cinnabarinus, and $M$. lactericeus); and $M$. lactericeus is said to grow best at 37 degrees. None of these characters seem to us from the descriptions to be of specific importance. Certain differences in the chemical nature of the pigment produced are cited by Migula (I900). These observations are suggestive, but require extension and correlation before they can be interpreted from the systematic standpoint.

Two other forms, sometimes included with the species listed above, are the $M$. prodigiosus of Cohn and the organism isolated by Babes from red sweat and named M. hamatodes. The former is now known to be a short bacillus, and the latter also, according to the original description, appears to be a rod form.

There remains to be considered one well-marked, but exceptional, form among the red cocci, the form characterized by the exhibition of motility. This was first described by Ali-Cohen (I889) under the name Micrococcus agilis. It was a slow-growing coccus, isolated from water, forming a pinkish growth mass and gradually liquefying gelatin. There can be little doubt from AliCohen's description that he was dealing with a truly motile organism. A single flagellum could be demonstrated by appropriate stains. Lehmann and Neumann (I896) failed to find either flagella or motility in their 
cultures of $M$. agilis. Migula, on the other hand, shows in Plate III of his System der Bakterien (I900) an excellent photo-micrograph of a flagellated culture of $M$. agilis. Hinterberger (I904), too, has published photo-micrographs of flagella in a culture of $M$. agilis over ten months old. Motility had been lost at this time, but the persistence of flagella indicated a characteristic difference from other forms.

An organism identical with $M$. agilis, except for the occurrence of the cells in packets, was described by Maurea in 1892 under the name Sarcina mobilis. Migula (1897) observed well-marked packets of eight to sixty-four cells in cultures of $M$. agilis when grown on moist agar; and he classes both forms in his genus Planosarcina.

There seems no doubt. from these observations, and from the experience of many bacteriologists with stock cultures of $M$. agilis, that motile flagellated rhodococci are occasionally found. In the light of present knowledge, however, the property seems to be a rare and exceptional one. If this is the case, it seems best, from the viewpoint adopted in this revision, not to retain a specific name for these few peculiar strains. On the other hand, further study may show that the property is of sufficiently common occurrence to constitute a true type center of variation. In that case, the relation of the motile forms to $R$. roseus and $R$. fulvus can be determined by comparative studies, and a new specific type may be intelligently recognized. 
SUMMARY OF THE GENERA AND SPECIES OF THE COCCACEÆ.*

\section{Family Coccacere (Zopf) Migula.}

Cells, in their free condition, spherical; during division somewhat elliptical. In the latter condition, division has already set in, altho it may not be apparent. Division in one, two, or three planes, without previous elongation of the cells. If the cells remain in contact after division they are frequently flattened in the plane of division. Motility is present only in a few forms. Formation of endospores appears to be absent or very rare.

\section{Subfamily A. Paracoccacex Winslow and Rogers.}

Parasites (thriving only,- or best,- on, or in, the plant and animal body). Thrive well under anaerobic conditions. Many forms fail to grow on artificial media; none produce

* These characterizations of species and genera must be interpreted in the light of the views in regard to bacterial relationships set forth in Chapter I. They correspond to the central types, about which other varieties of the cocci are grouped; but intermediate varieties will frequently be met with.

The meaning of the terms used in the descriptions is fully explained in Chapters III and IV. The standard color chart for determining chromogenesis is reproduced as the frontispiece of this volume. The quantitative relations of each genus in regard to acid production, gelatin liquefaction, etc., are indicated in the table on page 97 and in Figures I-III. 
very abundant surface growths. Planes of fission often parallel, producing pairs, or short or long chains, never packets. Generally stain by Gram. Produce acid in dextrose and lactose broth. Pigment, if any, white or orange.

Genus I. Diplococcus (Weichselbaum) Winslow and Rogers.

Strict parasites, not growing, or growing very poorly, on artificial media. Cells normally in pairs, surrounded by a capsule. Fermentative powers high, most strains forming acid in dextrose, lactose, saccharose, and inulin. Hemolytic power generally lacking. Characteristic group serum reactions.

\section{Species I. D. pneumonia Weichselbaum.}

A coccus occurring commonly in the body, in pairs of elongated lanceolate cells, surrounded by a capsule. Stains by Gram. On media often in short chains. Growth on media faint, producing small translucent disklike surface colonies. Ferments monosaccharides, disaccharides, and inulin. Lacks hemolytic power. Virulence very variable. Shows characteristic group serum reactions. Occurs in normal or pneumonic sputum and in infected tissues in pneumonia.

\section{Species 2. D. gonorrhœe (Neisser) Flügge.}

A coccus occurring commonly in pairs of flattened coffeebean shaped cells. Decolorizes by Gram. Fails to 
develop, or develops very feebly, on ordinary media. Not pathogenic for animals. Occurs most abundantly in pus cells in gonorrheal infections. Exhibits group serum reactions with $D$. Weichselbaumii.

Species 3. D. Weichselbaumii (Weichselbaum) Trevisan.

A coccus occurring commonly in pairs of flattened coffeebean shaped cells. Decolorizes by Gram. Develops feebly on ordinary media. Slightly pathogenic for animals. Occurs in cerebro-spinal exudate in epidemic meningitis. Exhibits group serum reactions with $D$. gonorrhoee.

Species 4. D. catarrhalis Frosch and Kolle.

A coccus occurring commonly in pairs of flattened coffeebean shaped cells. Decolorizes by Gram. Grows fairly well on artificial media, forming grayish-white colonies with a denser center. Growth on streak dry and friable. Virulence slight. Occurs in normal throat.

\section{Species 5. D. involutus (Kurth) Winslow.}

A coccus occurring commonly in chains, the elements of which are grouped in pairs. In the body, and on media, the chains are surrounded by a well-marked capsule. Growth on media fairly abundant, moist and slimy. Ferments monosaccharides, disaccharides, and inulin. Lacks hemolytic power. Shows group serum reactions with $D$. pneumonia. Occurs on the epithelial surfaces, and in the tissues, of man and the higher animals. 
Genus II. Ascococcus (Cohn) Winslow and Rogers. Saprophytic, growing vigorously in saccharine solutions. Cells in pairs, or in chains of paired elements. In presence of certain carbohydrates large, lobed gelatinous masses of zooglea formed. Fermentative powers high, acid being produced in dextrose, lactose, and saccharose.

Species I. Asc. mesenteroides Cienkowski.

A coccus occurring commonly in pairs, or chains of paired elements. On media not containing sugar, produces faint translucent colonies; on media containing saccharose, forms large, lobed masses of zooglea. Produces acid in dextrose, lactose, saccharose, and dextrin. Develops best between 30 and 37 degrees. In zooglea stage highly resistant to dry heat.

Genus III. Streptococcus (Billroth) Winslow and Rogers.

Parasites. Cells normally in short or long chains (under unfavorable cultural conditions, sometimes in pairs and small groups, never in large packets). Generally stain by Gram. On agar streak, effused translucent growth, often with isolated colonies. In stab culture, little surface growth. Sugars fermented with formation of large amount of acid. Generally fail to liquefy gelatin or reduce nitrates.

Species I. Str. equinus Andrewes and Horder.

This type of streptococcus appears to be characteristic of the intestine of the herbivora. It is abundant in horse 
dung and is the commonest form in the air of London. It forms chains of medium length, grows feebly, if at all, at 20 degrees, and ferments saccharose and the glucosides (salicin and coniferin) but not lactose, raffinose, inulin, or mannite. It fails to clot milk or to reduce neutral red.

\section{Species 2. Str. mitis Andrewes and Horder.}

This type is found most commonly in human saliva and fæces, but is not as a rule associated with disease. It is short-chained, grows well on gelatin at 20 degrees, and acidifies milk without clotting. It ferments lactose as well as saccharose and salicin, but gives a negative reaction to the other tests.

Species 3. Str. pyogenes Andrewes and Horder.

This type represents the highest parasitic development of the group, being rarely found except in association with definite pathological conditions. It occurs in long chains, usually growing in woolly masses at the bottom of a clear broth. It grows well on gelatin at 20 degrees. It is actively hemolytic, but does not form hydrogen sulphid in broth culture. It strongly acidifies milk, but never clots it, nor does it reduce neutral red. The usual positive reactions with Gordon's tests are saccharose, lactose, and salicin. It is highly pathogenic for animals.

Species 4. Str. salivarius Andrewes and Horder.

This type is the commonest form in the mouth, altho it is also found in the intestine. It is a short-chained form 
which usually renders broth uniformly turbid. Its growth on gelatin at 20 degrees is variable. It clots milk and reduces neutral red and ferments saccharose, lactose, and raffinose.

\section{Species 5. Str. anginosus Andrewes and Horder.}

This type is a pathogenic long-chained form, allied in other respects to Str. salivarius, and bearing to it much the same relation which $S t r$. pyogenes bears to Str. mitis. It occurs most commonly in cases of scarlatinal and other forms of sore throat. It is long-chained and produces a flocculent deposit in broth. It generally fails to grow on gelatin at 20 degrees, and is markedly hemolytic. Like Str. salivarius, on the other hand, it clots milk, reduces neutral red, and forms acid in saccharose, lactose, and raffinose.

Species 6. Str. facalis Andrewes and Horder.

This type is specially characteristic of the human intestine. It is short-chained and renders broth uniformly turbid. It grows readily at 20 degrees and forms sulphureted hydrogen in broth cultures. It has no hemolytic power and little virulence, but produces a positive reaction to all of Gordon's tests except raffinose and inulin. That is, it clots milk, reduces neutral red, and ferments saccharose, lactose, salicin, coniferin, and mannite. The mannite reaction is specially characteristic of this intestinal type. 
Species 7. Str. gracilis (Escherich) Lehmann and Neumann.

Small coccus occurring in chains. Ferments lactose and coagulates milk. May ferment salicin and mannite. Liquefies gelatin, actively.

Genus IV. Aurococcus Winslow and Rogers.

Parasites. Cells in groups and short chains, very rarely in packets. Generally stain by Gram. On agar streak good growth, of orange color. Sugars fermented with formation of moderate amount of acid. Gelatin often liquefied very actively. May or may not reduce nitrates.

Species I. Aur. aureus (Rosenbach) Winslow.

A parasitic coccus, living normally on the surface of the human or animal body, or in diseased tissues. Occurs singly, or in pairs, or irregular groups, rarely in short chains. Generally stains by Gram. Good to abundant surface growth, of orange color. Moderate acid production in dextrose and lactose broth. Nitrates not reduced. Growth good at both 20 degrees and 37 degrees, but pigment production much better at 20 degrees. Gelatin liquefied rapidly.

Species 2. Aur. aurantiacus (Schröter, Cohn) Winslow.

A parasitic coccus, living normally on the surfaces of the human or animal body, or in diseased tissues. Occurs 
singly, or in pairs, or irregular groups, rarely in short chains. Generally stains by Gram. Meager to good surface growth, of orange color. Acid production moderate in dextrose broth, but slight in lactose broth. Nitrates not reduced. Growth better at 37 degrees, but pigment production better at 20 degrees. Gelatin not liquefied.

Species 3. Aur. mollis (Dyar) Winslow.

A parasitic coccus, living normally on the surfaces of the human or animal body, or in diseased tissues; often found in the air. Occurs singly, or in pairs, or irregular groups, rarely in short chains. Reaction to Gram stain variable, more often positive than not. Good to abundant surface growth, of orange color. Acid production moderate in dextrose and lactose broth. Nitrates reduced to ammonia, or nitrites, or both. Growth generally equal at 20 and 37 degrees; pigment production equal, or better at 20 degrees. Gelatin usually liquefied rapidly; rarely, not liquefied.

Genus V. Albococcus Winslow and Rogers.

Parasites. Cells in groups and short chains (never in packets). Generally stain by Gram. Growth on agar streak abundant, and porcelain-white in color. Sugars fermented with production of a moderate amount of acid. Gelatin liquefaction and nitrate reduction may or may not occur. 
Species r. Alb. pyogenes (Rosenbach) Winslow.

A parasitic coccus, living normally on the surfaces of the human or animal body, or in diseased tissues. Occurs singly, or in pairs, or irregular groups. Generally stains by Gram. Good to abundant white surface growth. Moderate acid production in dextrose and lactose broth. Nitrates not reduced. Growth best at 37 degrees. Gelatin liquefied, but somewhat slowly.

Species 2. Alb. epidermidis (Gordon) Winslow.

A parasitic coccus, living normally on the surfaces of the human or animal body. Occurs singly, or in pairs, or irregular groups. Generally stains by Gram. Good to abundant, white surface growth. Moderate acid production in lactose, maltose, and glycerin media. Nitrates and neutral red reduced. Gelatin liquefied, somewhat slowly. Mannite not fermented,

\section{Species 3. Álb. candidus ('̉ohn) Winslow.}

A parasitic coccus, living normally on the surfaces of the human or animal body. Occurs singly, or in pairs, or irregular groups. Generally stains by Gram. Good to abundant, white surface growth. Moderate acid production in dextrose, lactose, and maltose media, not in glycerin or mannite. Nitrates not reduced. Gelatin not liquefied.

\section{Species 4. Alb. tetragenus (Gaffky) Winslow.}

A parasitic coccus, living normally on the surfaces of the human or animal body (most commonly in the nose and 
throat), or penetrating the tissues in disease. Occurs in the body in regular groups of four, surrounded by a capsule. Generally stains by Gram. Good surface growth, of grayish white color, and viscid consistency. Gelatin not liquefied.

\section{Subfamily B. Metacoccace}

Facultative parasites or saprophytes. Thrive best under aerobic conditions. Grow well on artificial media, producing abundant surface growths. Planes of fission often at right angles; cell aggregates in groups, packets, or zooglea masses. Generally decolorize by Gram. Pigment yellow or red.

Genus VI. Micrococcus (Hallier, Cohn) Winslow and Rogers.

Facultative parasites or saprophytes. Cells in plates or irregular masses (never in long chains or packets). Generally decolorize by Gram. Growth on agar abundant, with formation of yellow pigment. Dextrose broth slightly acid, lactose broth generally neutral. Gelatin frequently liquefied. Nitrates may or may not be reduced.

\section{Species I. M. flavus (Flügge) Migula.}

A saprophytic or semi-parasitic coccus, found most commonly in earth, water, and air, frequently also on the surfaces of the animal body. Occurs singly, or in pairs, or irregular groups. Generally decolorizes by Gram. Good to abundant, yellow surface growth. Reaction in dextrose 
broth faintly acid, in lactose broth alkalin or faintly acid. Gelatin slowly liquefied. Nitrates not reduced.

\section{Species 2. M. citreus (Dyar) Winslow.}

A saprophytic or semi-parasitic coccus, found most commonly in earth, water, and air, frequently also on the surfaces of the animal body. Occurs singly, or in pairs, or irregular groups. Generally decolorizes by Gram. Good to abundant, yellow surface growth. Reaction in dextrose broth faintly acid, in lactose broth alkalin or faintly acid. Gelatin slowly liquefied. Nitrates reduced to nitrites or ammonia.

\section{Species 3. M. luteus (Cohn) Migula.}

A saprophytic or semi-parasitic coccus, found most commonly in earth, water, and air, frequently also on the surfaces of the animal body. Occurs singly, or in pairs, or irregular groups. Variable reaction to Gram stain. Good to abundant, yellow surface growth. Reaction in dextrose broth faintly acid, in lactose broth alkalin or faintly acid. Gelatin not liquefied. Nitrates not reduced.

\section{Species 4. $M$. candicans Flügge.}

A saprophytic or semi-parasitic coccus, found most commonly in earth, water, and air, frequently also on the surfaces of the animal body. Occurs singly, or in pairs, or irregular groups. Variable reaction to Gram stain. Good to abundant growth, of white color. Fails to form 
acid in lactose, maltose, and glycerin media, but does acidify mannite. Gelatin not liquefied. Nitrates not reduced.

\section{Genus VII. Sarcina (Goodsir) Winslow and Rogers.}

Facultative parasites or saprophytes. Division occurs under favorable conditions in three planes, producing regular packets. Generally decolorize by Gram. Growth on agar abundant, with formation of yellow pigment. Dextrose broth slightly acid, lactose broth generally neutral. Gelatin frequently liquefied. Nitrates may or may not be reduced.

\section{Species I. S. flava De Bary.}

A saprophytic or semi-parasitic coccus, found most commonly in earth, water, and air, also on the surfaces of the animal body. Occurs under favorable conditions in packets. Generally decolorizes by Gram. Good to very heavy surface growth, of yellow color. Reaction in dextrose broth faintly acid, in lactose broth alkalin or faintly acid. Gelatin slowly liquefied. Nitrates not reduced.

\section{Species 2. S. citrea Winslow.}

A saprohytic or semi-parasitic coccus, found most commonly in earth, water, and air, frequently also on the surfaces of the animal body. Occurs under favorable conditions in packets. Generally decolorizes by Gram. Good to very heavy surface growth, of yellow color. 
Reaction in dextrose broth faintly acid, in lactose broth alkalin or faintly acid. Gelatin slowly liquefied. Nitrates reduced to nitrites or ammonia.

\section{Species 3. S. lutea Schröter.}

A saprophytic or semi-parasitic coccus, found most commonly in earth, water, and air, frequently also on the surfaces of the animal body. Occurs under favorable conditions in packets. Generally decolorizes by Gram. Good to abundant, yellow surface growth. Reaction in dextrose broth faintly acid, in lactose broth alkalin or faintly acid. Gelatin not liquefied. Nitrates not reduced.

\section{Genus VIII. Rhodococcus Winslow and Rogers.}

Saprophytes. Cells in groups or regular packets. Generally decolorize by Gram. Growth on agar abundant, with formation of red pigment. Dextrose broth slightly acid, lactose broth neutral. Gelatin rarely liquefied. Nitrates generally reduced to nitrites, but not to ammonia.

\section{Species I. R. roseus (Flügge, Dyar) Winslow.}

A saprophytic coccus, found most commonly in earth, water, and air. Occurs in pairs, groups, or packets. Generally decolorizes by Gram. Abundant, red surfäce growth. Reaction in dextrose broth faintly acid, in lactose broth alkalin or faintly acid. Gelatin either not liquefied or liquefied very slowly. Nitrates reduced to nitrites, but not to ammonia. 
Species 2. $R$. fulvus (Cohn) Winslow.

A saprophytic coccus, found most commonly in earth, water, and air. Occurs in pairs, groups, or packets. Generally decolorizes by Gram. Abundant, red surface growth. Reaction in dextrose broth faintly acid, in lactose broth alkalin or faintly acid. Gelatin either not liquefied or liquefied very slowly. Nitrates not reduced. 


\section{KEY TO THE GENERA AND SPECIES OF THE COCCACE $A$}

CELLS SPHERICAL: FAMILY, COCCACEÆ.

A. Parasites. Growth not Abundant, (or, one Species, Zooglea-Forming Saprophytes. Growth Abundant IN Saccharose Media). Generally Gram +. Acid FORMERS.

\section{Subfamily, Paracoccaceer.}

I. Cells in capsulated pairs. Parasites. Growth very meagre. Inulin fermented. No pigment.

Genus, Diplococcus.

A. Cells lanceolate, in pairs, no chains. Gram +.
I. In lungs and sputum.
D. pneumonice.

B. Cells flattened, in pairs, no chains. Gram -.

2. In pus cells in gonorrheal infection. Growth, meagre. $\quad D$. gonorrheee.

3. In cells in cerebro-spinal exudate. Growth meagre.

D. Weichselbaumii.

4. In normal throat. Growth on media fair.

D. catarrhalis.

C. Cells in chains of pairs, surrounded by zooglea.

5 .

D. involutus.

II. Cells in chains occurring in masses of zooglea in sugar refineries. Aberrani saprophytic forms. Growth abundant in saccharose media. No pigment.

Genus, Ascococcus.

I.

A. mesenteroides. 
III. Cells in chains. Parasites. Growth meagre. Inulin not fermented. No pigment. Genus, Streptococcus.

A. Does not liquefy gelatin.

a. Does not acidify lactose.

I. Chains medium. Growth at 20 degrees feeble. Habitat, intestines of Herbivora. Acidifies saccharose, salicin, and coniferin.

Str. equinus.

b. Acidifies lactose, saccharose, and salicin but not raffinose. Does not clot milk.

2. Chains short. Habitat, human saliva and fæces. Str. mitis.

3. Chains long. Hemolytic power marked. Found in pathological conditions.

Str. pyogenes.

c. Acidifies lactose, saccharose, and raffinose but not salicin. Clots milk. 'Reduces neutral red.

4. Chains short. Habitat, normal mouth and intestine.

Str. salivarius.

5. Chains long. Hemolytic power marked. Found in cases of sore throat.

Str. anginosus.

d. Acidifies lactose, saccharose, salicin, coniferin, and mannite but not raffinose. Clots milk. Reduces neutral red.

6. Chains short. Habitat, human intestine. Str. facalis.

B. Liquefies gelatin. 
IV. Cells in irregular groups. Parasites. Growth fair. Orange pigment. Genus, Aurococcus.

A. Nitrates not reduced.

I. Gelatin strongly liquefied.

2. Gelatin not liquefied.

$$
\text { Aur. aureus, }
$$

Aur. aurantiacus.

B. Nitrates reduced.

3.

Aur. mollis.

V. Cells in irregular groups or in fours. Parasites. Growth good. White pigment. Genus, Albococcus.

A. Cell groups irregular.

a. Gelatin liquefied.

r. Nitrates not reduced.

2. Nitrates reduced.

Alb. pyogenes.

Alb. epidermidis.

b. Gelatin not liquefied.

3.

Alb. candidus.

B. Cells in the body in capsulated groups of four. Growth viscid.

4.

Alb. tetragenus.

B. Saprophytes. Growth Abundant. No Zooglea. Gram Generally -. Not Acid Formers. Subfamily, Metacoccacez.

VI, Cells in irregular groups. Pigment generally yellow.

\section{Genus, Micrococcus.}

A. Pigment typical, yellow.

a. Gelatin liquefied.

r. Nitrates not reduced.

M. flavus.

2. Nitrates reduced.

b. Gelatin not liquefied. $\quad$ M. citreus.

M. luteus. 
266 RELATIONSHIPS OF THE COCCACE

B. Pigment white. Gelatin not liquefied. Nitrites not reduced.

4.

VII. Cells in packets. Pigment yellow.

A. Gelatin liquefied.

I. Nitrates not reduced.

2. Nitrates reduced.

IB. Gelatin not liquefied.

3.
M. candicans.

(Candéo: tobe of a

Genus, Sarcina.

S. flava.

S. citrea. 


\section{BIBLIOGRAPHY}

Publications marked with an asterisk were not accessible to the authors and are cited from the references of others.

Albrecht, H., and Ghon, A. 1902. Zur Frage der morphologischen und biologischen Charakterisierung des meningococcus intracellularis. Centralblatt für Bakteriologie, Abteilung I, Originale, XXXIII, 496.

Ali-CoHEN, C. H. r889. Eigenbewegung bei Mikrokokken. Centralblatt für Bakteriologie, VI, 33.

Andrewes, F. W. rgo6. The Evolution of the Streptococci. Lancet, I906, II, I4.15.

Andrewes, F. W., and Horder, T. J. I906. A Study of the Streptococci Pathogenic for Man. Lancet, r906, II, 708.

Aronson, H. r903. Weitere Untersuchungen über Streptococcen.

Deutsche Medicinische Wochenschrift, XXIX, 43.9.

BABES, V. r903. Das maltafieber Kolle und Wassermann. Handbuch der pathogenen mikroorganismen, III, 438 .

Baginsky, A. 1896. Die Anwendung des AntistreptokokkenSerum (Marmorek's) gegen Scharlach. Verhandlungen der Berliner medicinischen Gesellschaft, XXVII, II, г16.

BAgINsky, A. I902. Ueber Antistreptokokken-Serum bei Scharlach. Berliner klinische Wochenschrift, XXXIX, IrI3.

Baginsky, A., and Sommerfeld, P. Igoo. Ueber einen constanten Bacterienbefund bei Scharlach. Berliner klinische Wochenschrift, XXXVII, $5^{88 .}$

BARUChello, L. 'Tg05. Untersuchungen über die Darmstreptokokken des Pferdes. Centralblatt fïr Bakteriologie, Abteilung, I, Originale, XXXIX, 569. 
Beaton, R. M., and Walker, E. W. A. I903. The Etiology of Acute Rheumatism and Allied Conditions. British Medical Journal, I903, I, 237.

BeAtTiE, J. M. Igo6. Experimental work in relation to Micrococcus rheumaticus and streptococcus pyogenes. Journal of Medical Research, XIV, 399.

BEHRING, I892. Untersuchungsergebnisse betreffend den streptococcus longus. Centralblatt für Bakteriologie, XII, I92.

Berry, J. L. 1907. A study of the Pneumococcus, with Especial Reference to the Inulin Test. Journal of Infectious Diseases, IV, 93 .

BERTARELII, E. I906. Ueber einem pathogenen Keim der Iguana und interessante, von ihm erzeugte Verletzungen (Diplococcus iguanæ n. sp.). Centralblatt für Bakteriologie, Abteilung I, Originale, XL, $45^{8}$.

v. BESSER, L. I889. Ueber die Bakterien der normalen Luftwege, Beiträge zur pathologischen Anatomie und allgemeinen Pathologie, VI, 33I.

Bettencourt, A., and Franca, C. I904. Ueber die Meningitis cerebrospinalis epidemica und ihren spezifischen Erreger. Zeitschrift für Hygiene, XLVI, 463.

* Beyerinck, M. W. Ig00. On different Forms of Hereditary Variation of Microbes. Königlichen Akadamie von Wetenschappen te Amsterdam, 27 Okt. I900.

BEYERINCK, M. W. 1905. Een obligaat anäerobe gistingssarcine. (Ref.) Centralblatt für Bakteriologie, Abteilung II, $\mathrm{XV}, 473$.

Bigelow, R. P. I904. Article on "Variation," Wood's Reference Handbook of the Medical Sciences, VIII, I904, 182.

* Billroth, T. i874. Untersuchungen über die Vegetationsformen von Coccobacteriea septica und den Antheil, welchen sie an der Entstehung und Verbreitung der accidentelen Wundkrankheiten hablen. Berlin, 1874 . 
BINAGHI, R. I897. Ueber einen Streptococcus capsulatus. Centralblatt für Bakteriologie, Abteilung, I, XXII, 273.

BIRge, E. G. rgo5. Some Observations on the Occurrence of Micrococcus zymogenes. Johns Hopkins Hospital Bulletin XVI, 309.

BOXER, S. I906. Ueber das Verhalten von Streptokokken und Diplokokken auf Blutnährböden, Centralblatt für Bakteriologie, Abteilung I, Originale, XL, 59 I.

BRUCE, D. I893. Sur une nouvelle forme de fièvre rencontrée sur les bords de la Méditerrannée. Annales de l'institut Pasteur, VII, 289.

Buterger, L. I904. A New Method for Staining the Capsules of Bacteria. Medical News, LXXXV, iп 7.

BUERger, L. I905; a. The macroscopic indentification of colonies of the Pneumococcus. Centralblatt für Bakteriologie, Abteilung I, Originale, XXXIX, 20.

BUERger, L. I905; b. Studies of the pneumococcus and allied organisms with reference to their occurrence in the human mouth. Journal of Experimental Medicine, VII, 497.

BUERger, L. I906. Beitrag zur Kenntnis des Streptococcus mucosus capsulatus. Centralblatt für Bakteriologie, Abteilung I, Originale, XLI, $3^{\mathrm{I}} 4$.

Bumm, E. 1884. Beitrag zur Kenntniss der Gonorrhoe der weiblichen Genitalien. Archiv für Gyncekologie, XXIII, 327.

Bumm, E. 1885. Der Mikro-Organismus der Gonorrhoischen Schleimhaut Erkrankungen Gonococcus-Neisse. Wiesbaden, I885.

BÜ̈RG, M. I905. Die Staphylokokkeninfektion bei den Hasen. Centralblatt für Bakteriologie, Abteilung I, Originale XI, 9 I.

BURRI, R. I904. Ueber einen Schleimbildenden Organismus aus der Gruppe des Bacterium Güntheri u.s.w. Centralblatt für Bakteriologie, Abteilung II, XII, 37r. 
Catterina, G. Ig03. Ueber eine bewimperte Micrococcusform, welcher in einer Septikämie der Kaninchen gefunden wurde. Centralblatt für Bakteriologie, XXXIV, Io8.

Celler, H. L. Igo2. Demonstrations of Cultures of the Meningococcus and Micrococcus catarrhalis. Proceedings of the New York Pathological Society, II, I28.

Chester, F. D. Igor. A Manual of Determinative Bacteriology. New York, I90I.

Chester, F. D. Ig04. Observations on an Important Group of Soil Bacteria. Fifteenth Annual Report of the Delaware College Agricultural Experiment Station, for 1903.

* Cienkowskr, L. I878. Die Gallertbildungen des Zuckerrübensaftes. Charkow, 1878 .

Clark, H. W., and Gage, S. D. I905. The Functions of Various Types of Bacteria in the Purification of Sewage, with some Methods for their Quantitative Determination. Engineering News, LIII, 27.

Class, W. J. I899. The Etiology of Scarlet Fever. Medical Record, LVI, 330.

Claussen, N. H. I903. Ueber die Sarcinakrankheit des Bieres und ihre Erreger. Centralblatt für Bakteriologie, Abteilung II, $\mathrm{X}, 56 \mathrm{I}$.

Corn, F. I872. Untersuchungen über Bacterien. Beiträge zur Biologie der Pflanzen, I, Heft II, I27.

Corn, F. I875. Untersuchungen über Bacterien, II. Beiträge zur Biologie der Pflanzen, I, Heft III, I4I.

Cole, R. I. I904. Experimental Streptococcus Arthritis in Relation to the Etiology of Acute Articular Rheumatism. Journal of Infectious Diseases, I, 7r4.

Collins, K. R. I905. The Application of the Reaction of Agglutination to the Pneumococcus. Journal of Experimental Medicine, VII, 420. 
Committee on Standard Methods of Water Analysis, 1905. Report to the Laboratory Section of the American Public Health Association. Journal of Infectious Diseases, Supplement No. I, I, May, I905.

Conn, H. W. Igoo. Natural Varieties of Bacteria. Journal of the Boston Society of Medical Sciences, IV, I 70.

Courmont, J. Igo6. Staphylococcie Nouveau traité de médicine et de thérapeutique. Publié sous la direction de MM. P. Brouardel et A. Gilbert. Fas. X. Paris, Igo6.

Dammann and Manegold. Igo6. Die Schlafkrankheit der Hüh ner. Eine durch einen Kapselstreptococcus (Strept. capsulatus gallinarum) hervorgerufene Hühnerseuche. (Ref.) Centralblatt für Bakteriologie, Abteilung I, Referate XXXIX, 60.

De Waele and Sugg, I903. Etude sur la variole et la vaccine. Archives internationales de Pharmacodynamie et de Therapie XII, 205 .

Dudgeon, L. S. Igo8. The Differentiation of the Staphylococci. Journal of Pathology and Bacteriology, XII, 242.

Dunham, E. K. I903. The Influence of Physical Conditions on the Character of Colonies on Gelatin Plates. Abstract, Science, N.S. XVII, 372 .

DunHam, E. K. I906. Comparative studies of diplococci decolorized by Gram's method, obtained from the spinal fluid and from the nares of cases of epidemic cerebro-spinal meningitis. Journal of Infectious Diseases, Supplement No. II. Feb. 1906.

Duval, C. W., and Lewis, P. A. I905. Studies on the Pneumococcus, Journal of Experimental Medicine, VII, 473.

Dyar, H. G. 1895. On Certain Bacteria from the Air of New York City. Annals of the New York Academy of Sciences, VIII, 322.

Ellis, D. I902. Untersuchungen über Sarcina, Streptococcus and Spirillum. Centralblatt für Bakteriologie, Abteilung I, Originale, XXXIII, $\mathrm{x}$. 
*Escherich. I886. Die Darmbakterien des Säuglings und ihre Beziehungen zur Physiologie der Verdauung. Stuttgart, r886. Eyre, J. W., Leathem, A. N., and Washbourn, J. W. I906. A study of different strains of pneumococci, with especial reference to the lesions they produce. Journal of Pathology and Bacteriology XI, 246.

Fehleisen, 1883. Aetiologie d. Erysipels. Berlin. I883.

Fischer, H. I904, Die Bedeutung der Agglutination zur Diagnose der pathogenen und saprophytischen Streptokokken. Centralblatt fiur Bakteriologie, XXXVII, Abteilung I, Originale, 449.

Fıய̈GGE, C. I886. Die Mikroorganismen. Leipzig, I886.

FLüGGE, C. I896. Die Mikroorganismen. Auflage II. Leipzig, I896.

Fraenkel, C., and Baumann. I905. Ueber Hämolysinbildung und Agglutination der Staphylokokken. Münchener medizinische Wochenschrift, LII, 937.

FrIEdL ÄNDER, C. I883. Die Mikrokokken der Pneumonie. Fortschritte der Medicin, I, 7I5.

Frosch, P., and Kolle, W. r896. Die mikrokokken. Flügge's Die Mikroorganismen. I896, II, 96.

Fuller, G. W., and Johnson, G. A. I899. On the Differentiation and Classification of Water Bacteria. Journal of Experimental Medicine, IV. 6og.

GAFFKY, I883. Ueber antiseptische Eigenschaften des in der Esmarchschen Klinik als Verbandmittel benutzten Torfmulls. Archiv für klinische Chirurgie, XXVIII, 495.

Gage, S. DeM. Ig05. Contribution to the Biochemistry of Sewage Purification; the Bacteriolysis of Peptones and Nitrates. Journal of American Chemical Society, XXVII, 327.

Gage, S. DeM., and Phelps, E. B. I903. On the Classification and Identification of Bacteria, with a Description of the Card System in use at the Lawrence Experiment Station for Records 
of Species. Transactions of the American Public Health Association, XXVIII, 1902 meeting, 494.

Galton, F. I889. (a) Co-relations and their Measurement, chiefly from Anthropometric Data. Proceedings of the Royal Society. XLV, r889, I35. (b) Natural Inheritance. London, I889.

Ghon, A., Pfeiffer, H., and Sederd, H. I902. Der Mikrococcus catarrhalis (R. Pfeiffer) als Krankheitserreger. Zeitschrift für klinische Medicin, XLIV, 262.

*GoodsIR. I842. History of a case in which a fluid periodically ejected from the stomach contained vegetable organisms of an undescribed form. Edinburgh Medical and Surgical Journal, April, 1842.

Gordon, M. H. I90I. Further Report on the Bacteriology of Scarlatina, with Special Reference to the Investigation of Ten Fatal Examples of that Disease. Supplement to the Twentyninth Annual Report of the Local Government Board containing the Report of the Medical Officer for I899-1900, 385 .

GoRdon, M. H. rgo4. Report on a bacterial test for estimating pollution of air. Supplement to the Thirty-second Annual Report of the Local Government Board, containing the Report of the Medical Officer for 1902-3, 42I.

Gordon, M. H. 1905. Report of some characters by which various streptococci and staphylococci may be differentiated and identified. Supplement to the Thirty-third Annual Report of the Local Government Board containing the Report of the Medical Officer for $1903-4,388$.

Gordon, M. H. Igo6. Report on Bacterial Test whereby Particles Shed from the Skin may be Detected in Air. Supplement to the Thirty-fourth Annual Report of the Local Government Board containing the Report of the Medical Officer for 1904-5, 387.

Gotschlich, E. r903. Allgemeine Morphologie und Biologie der pathogenen Mikroorganismen. Kolle und Wassermann, Handbuch der pathogenen Mikroorganismen, I, Jena, I903, 29. 
Gruber, T. I897. Die Arten der Gattung Sarcina. Arbeiten aus dem bacteriologischen Institut der technischen Hochschule zu Karlsruhe, I, 24I.

Gruber, T. I902. Beitrag zur Kenntnis der Erreger der schleimigen und fadenziehenden milch und charakterisierung des coccus lactis viscosi. Centralblatt für Bakteriologie, Abteilung II, IX, 785 .

* Hall, H. M. I907. Compositæ of Southern California. University of California Publications, Volume III, No. I.

Наsнтмото, S. IgoI. Zwei neue milchsäurebildende Kugelbakterien. Hygienische Rundschau, XI, 82I.

Heinemann, P. G. Igo6. The significance of streptococci in milk. Journal of Infectious Diseases, III, I73.

Hennemann, P. G. I907. The pathogenicity of Streptococcus lacticus. Journal of Infectious Diseases, IV, 87 .

HENRICI, H. I897. Beitrag zur Bakterienflora des Käses. Arbeiten aus dem bacteriologischen Institut der technischen Hochschule zu Karlsruhe, I, I.

Heyrovský, J. I905. Ein Beitrag zur Biologie und Agglutination des Diplococcus pneumoniæ. Centralblatt für Bakteriologie, Abteilung I, Originale, XXXVIII, 704.

Hinterberger, A. I904. Geisslen bei einer 5 Monate alten Proteuskultur und einer 10 $\frac{1}{2}$ Monate alten Kultur von Micrococcus agilis. Centralblatt für Bakteriologie, Abteilung I, Originale, XXXVI, 480 .

Hiss, P. H. Igo2. Beitrag zur physiologischen Differenzierung des Pneumococcus und des Streptococcus und zu den Methoden der Kapselfärbung. Centralblatt für Bakteriologie, Abteilung I, Referate, XXXI, 302.

Hiss, P. H. I905. A comparative study of pneumococci and allied organisms. Journal of Experimental Medicine, VII, 547 .

Hlava, I902. Lenconostoc hominis und seine Rolle bei den akuten exanthematischen Krankheiten (Scharlach, Masern, Fleck- 
typhus). Centralblatt für Bakteriologie, Abteilung, I, Originale, XXXII, 263.

HölLING, A. I905. Das Verhältnis der Milchsäurebakterien, zum Streptococcus lanceolatus. (Ref.) Centralblatt für Bakteriologie, Abteilung I, Referate, XXXVI, 659.

Horrocks, W. H. Igo3. The Bacillus coli communis; considered as an Indication of Sewage Contamination of Water Supplies. Journal of the Royal Army Medical Corps, I, 362 .

Houston, A. C. I905; a. Report on the Bacteriological Examination of (I) the Normal Stools of Healthy Persons; (2) the Intestinal Contents of Sea-fowl and Fish; and (3) Certain of our Public Water Supplies. Supplement to the Thirty-third Annual Report of the Local Government Board containing the Report of the Medical Officer for 1903-4, 472.

Howard, W. T., and Perkins, R. G. Igor. Streptococcus mucosus (Nov. Spec.?) Pathogenic for man and animals. Journal Medical Research, VI, I63.

Howe, F. 1904. Notes on the Bacillus coli. Centralblatt für Bakteriologie, Abteilung I, Originale, XXXVI, 484.

JAEGER, H. I902. Zur Frage der morphologischen und biologischen Charakterisierung des Meningococcus intrucellularis. Centralblattfïr Bakteriologie, Abteilung I, Originale, XXXIII, 23.

JAEger, H. I903. Die specifische Agglutination der Meningokokken als Hilfsmittel zu ihrer Artbestimmung und zur bakteriologischen Diagnose der epidemischen Genickstarre. Zeitschrift für Hygiene, XLIV, 225.

Jordan, D. S., and KellogG, V. L. 1907. Evolution and Animal Life. New York, I907.

JORDAN, E. O. r903. The kinds of Bacteria found in River Water. Journal of Hygiene, III, I.

Kayser, H. I902. Die Einwirkung des Traubenzuckers auf verschiedene Lebensäusserungen des Staphylococcus pyogenes (Virulenz, Hämolysin u. sw.). Zeitschrift für Hygiene, XI, 2I. 
Kendall, A. I. I903. A proposed Classification and Method of Graphical Tabulation of the Characters of Bacteria. Transactions of the American Public Health Association, XXVIII, I902 meeting, 48r.

KerNer, J. I905. Experimenteller Beitrag zur Hämolyse und zur Agglutination der Streptokokken. Centralblatt für Bakteriologie, Abteilung I, Originale, XXXVIII, 223.

KINDBORg, A. I905. Die Pneumokokken. Zeitschrift für Hygiene LI, I97.

KLOPSTOCK and BockENHEIMER. I903. Beitrag zur Agglutination der Staphylokokken. Archiv für klinische Chirurgie, LXXII, 325.

KNORR, I893. Experimentelle Untersuchungen über den Streptococcus longus. Zeitschrift fiir Hygiene, XIII, 427.

Kolle, W., and Otto, R. Igo2. Die Differencirung der Staphylokokken mittelst der Agglutination. Zeitschrift für Hygiene, XLI, 369 .

KONRÁDI, D. Ig04. Ueber die Lebensdauer pathogener Bakterien im Wasser. Centralblatt für Bakteriologie, Abteilung I, Originale, XXXVI, 203.

KRUSE, W. I896. Variabilität der Mikroorganismen. Die Mikroorganismen; C. Flügge. III Auflage. Leipzig. I896, 475.

KRUSE, W. I903. Das Verhältnis der Milchsaürebakterien zum streptococcus lanceolatus. Centralblatt für Bakteriologie, Abteilung, I, Originale, XXXIV, 737.

Kruse, W., and Pansint, S. I892. Untersuchungen über den Diplococcus pneumoniæ und verwadte Streptokokken. Zeitschrift für Hygiene, XI, 279.

KuRTH, H. r89r. Ueber die Unterscheidung der Streptokokken und über das Vorkommen derselben, insbesondere des Streptococcus conglomeratus beim Scharlach. Arbeiten aus dem Kaiserlichen Gesundheitsamte, VII, 389. 
KurTH, H. I893. Bakteriologische Untersuchungen bei Maulund Klauenseuche. Arbeitan aus dem Kaiserlichen. Gesundheitsamte, VIII, 439.

KuTSCHER and KonRICH, F. Ig04. Untersuchungen über die Beziehungen von Hämolysinbildung und Agglutinabilität der Staphylokokken. Zeitschrift für Hygiene, XLVIII, 249.

LE Gros, F. L. Igo2. Monagraphie des streptocoques et des agents des septicémies métadiphtériques particulièrement des diplocoques. Paris, I902.

Lemmann, K. B., and Neumann, R. 1896. Atlas und Grundriss der Bakteriologie. Munich. 1896.

Leichmann, G. I896. Ueber die freiwillige Säuerung der Milch. Centralblatt für Bakteriologie, Abteilung II, II, 776.

LEPIERRE. Ig04. Subsidio para o estudo do meningococco. (Ref.) Centralblatt für Bakteriologie, Abteilung I, Referate, XXXV, 43 and ror.

Le Roy des Barres, A., et Weinberg, M. I899. Septicémie aiguë à streptocoque encapsulé. Archives de médecine expérimentale et d'anatomie pathologique, XI, 399.

LiBMAN, E. Igoo. I. Ueber einen neuen pathogenen Streptococcus. II. Ueber eine eigentümliche Eigenschaft (wenigstens mancher) pathogener Bakterien. Centralblatt für Bakteriologie, Abteilung I, XXVIII, 293.

LIBMAN, E. IgOI. On certain features of the growth of bacteria on media containing sugars and serum; with remarks upon the acid production. Journal of Medical Research, VI, 84.

LIBMAN, E. Ig02. Remarks on the Meningococcus and on its Relationship to the Gonococcus and Micrococcus catarrhalis. Proceedings of the New York Pathological Society, II, I34.

*Lindner. I888. Die Sarcina-Organismen der Gärungsgewerbe. Inaug.-Diss. Berlin. I888.

v. Lingelsheim. I89I. Experimentelle Untersuchungen über morphologische, culturelle und pathogene Eigenschaften verschiedener Streptokokken. Zeitschrift für Hygiene, X, 33I. 
v. Lingelsherm. rgo3. Streptokokken. Kolle und Wassermann's Handbuch der pathogenen mikroorganismen. Jena, I903, 303.

Loewenberg. I899. Une sarcine pathogène. Annales de l'institut Pasteur, XIII, $35^{8}$.

LONGCOPE, W. T. I902. Streptococcus mucosus (Howard) and its relation to micrococcus lanceolatus. Journal of Medical Research, VII, 220.

LongCOPE, W. T. I905. A note upon the growth of pneumococci and streptococci in blood serum. Journal of Experimental Medicine, VII, 626.

LongCope, W. T., and Fox, W. W. I905. A comparative study of pneumococci and streptococci from the mouths of healthy individuals and from pathological conditions. Journal of Experimental Medicine, VII, 430.

Lubinski, W. I894. Ueber die Anaërobiose bei der Eiterung. Centralblatt für Bakteriologie, XVI, 769.

MacCallum, W. G., and Hastings, T. W. I899. A case of acute endocarditis caused by Micrococcus zymogenes (Nov. spec.), with a description of the microorganism. Journal of Experimental Medicine, IV, $52 \mathrm{I}$.

Macchiati, I899. Sopra uno streptococco parassito dei granuli d'amido di frumenti Boll. societa, botanica, italiana. I899, 48 .

*MarmoreK, A. I895. Streptococcus und Antistreptokokkenserum. Wien. 1895 .

MARMOREK, A. I902; a. Das Streptokokken-Gift. Berliner klinische Wochenschrift, XXXIX, 253.

MARMOREK, A. I902; b. Die Art-Einheit der für den Menschen pathogenen Streptokokken. Berliner klinische Wochenschrift, XXXIX, 299.

MAssinI, R. Ig07. Über einen in biologischer Beziehung interessanten Kolistamm (Bacterium coli mutabile). Ein Beitrag zur Variation bei Bakterien. Archiv für Hygiene, LXI, 250. 
Meyer, A. Ig03. Practicum der botanischen Bakterienkunde. Jena. I903.

Meyer, F. I902. Zur Einheit der Streptokokken. Berliner klinische Wochenschrift, XXXIX, 936.

Migula, W. 1897. System der Bakterien. Band I. Jena. I897.

Migula, W. Ig00. System der Bakterien. Band II, Jena, I900.

Moberg, L., and UnNa, P. G. Igoo. Versuch einer botanischen Klassifikation der beim Ekzem gefundenen Kokkenarten nebst Bemerkungen über ein natürliches System der Kokken überhaupt. B. Spezieller Teil. Monatschefte fiir praktische Dermatologie, XXXI, 65.

*Molisch, H. I907. Die Purpurbakterien nach neueren Untersuchungen. Jena. 1907.

Moser, P. I902. Ueber die Behandlung des Scharlachs mit einem Scharlachstreptococcen-serum. Wiener klinische Wochenschrift, XV, I053.

Moser, P., and v. Pirquet. Igo2. Agglutination von Scharlachstreptococcen durch menschliches Serum. Agglutination von Streptococcen durch Pferdesera. Wiener klinische WWochenschrift, XV, 1086.

MUIR, R., and Ritchie, J. I903. Manual of Bacteriology. American Edition by N. MacL. Harris. New York. 1903.

NedrigaIlow, W. I906. Zur Frage über die spezifische Natur des Scharlachstreptococcus. Centralblatt für Bakteriologie, Abteilung, I, Originale, XLII, I3.

Neisser, A. I879. Ueber eine der Gonorrhoe eigentümliche Micrococcusform. Centralblatt für die medicinischen Wissenschaften, XVII, 497.

Neisser, M., and Wechsberg, F. Igor. Uber das Staphylotoxin. Zeitschrift für Hygiene, XXXVI, 299.

Neisser, M., and Lipstein, A. I903. Die Staphylokokken. Handbuch der pathogenen Mikroorganismen. Kolle and Wassermann. Band III. Jena. I903, I05. 
Neisser, M. 1906. Ein Fall von Mutation nach de Vries bei Bakterien und andere Demonstrationen. Centralblatt für Bakteriologie, XXXVIII, Abteilung I, Referate, Beiheft, 98.

Nenckr, M. I89r. Die isomeren Milchsäuren als Erkennungsmittel einzelner Spaltpilzarten. Centralblatt für Bakteriologie, IX, 304 .

Neufeld, F. I903. Ueber Immunität und Agglutination bei Streptokokken. Zeitschrift für Hygiene, XLIV, I6I.

Neumann, R. I897. Studien über die Variabilitắt der Farbstoffbildung bei Micrococcus pyogenes var aureus (Staphylococcus pyogenes aureus) und einigen anderen Spaltpilzen. Archiv für Hygiene, XXX, I.

Neumann, R. O. I904. Kapseltragende pathogene Streptokokken im Rachennasenraum. Centralblatt für Bakteriologie, Abteilung I, Originale, XXXVII, 48I.

Norris, C., and Pappenhermer, A. M. I905. A study of pneumococci and allied organisms in human mouths and lungs after death. Journal of Experimental Medicine, VII, $45^{\circ}$.

Oestern, K. I904. Beitrag zur Kenntnis der Bakterienflora der erweichten tuberkulösen Herde des Rindes. Centralblatt für Bakteriologie, Abteilung I, Originale, XXXVII, 178 .

Ogston, A. I880. Ueber Abscesse. Archiv für klinische Chirurgie, XXV, 588 .

Ortmann, A. E. 1908. The Distribution of closely Allied Species. Science, N. S., XXVII, 427.

Ortmann, P. I888. Beitrag zur Aetiologie der acuten Cerebrospinalmeningitis. Archiv fü experimentelle Pathologie und Pharmakologie, XXIV, 29 I.

Oтто, R. 1903. Weitere Beiträge zur Agglutination der Staphylokokken. Centralblatt für Bakteriologie, Abteilung I, Originale, XXXIV, 44 .

PAGE, C. G. I899. A preliminary study of streptococci isolated from throat cultures from patients ill with scarlet fever. Journal Boston Society of the Medical Sciences, III, 323. 
Park, W. H., and Williams, A. W. 1905. A study of pneumococci: a comparison between the pneumococci found in the throat secretions of healthy persons living in both city and country and those obtained from pneumonic exudates and diseased mucous membranes. Journal of Experimental Medicine, VII, 403.

Pasquale, A. I893. Vergleichende Untersuchungen über Streptokokken. Beiträge zur pathologischen Anatomie und zur allgemeinen Pathologie, XII, 433.

*PAsset. I885. Untersuchungen über die Aetiologie der eiterigen Phlegmone des Menschen. Berlin. 1885 .

Pearson, K. I900. The Grammar of Science. London. I900.

Peckham, A. W. 1897. The Influence of Environment upon the Biological Processes of the Various Members of the Colon Group of Bacilli. Journal of Experimental Medicine, II, 549.

Perkins, R. G., and Pay, G. O. I903. Streptococcus pyogenes in Variola. Journal of Medical Research, X, 180.

*Pitfield, R. L. I897. Queen's Microscopic Bulletin. Philadelphia. 1897 .

Poynton, F. J. and Paine, A. I900. The Etiology of Rheumatic Fever. Lancet, I900, II, 861.

Poynton, F. J. I904. The Infective Nature of Rheumatic Fever. British Medical Journal, I904, I, III 7 .

Prescott, S. C. I906. The Occurrence of Organisms of Sanitary Significance on Grains. Biological Studies by the pupils of William Thompson Sedgwick. Boston. I906, 208.

Pröscher. 1903. Ueber Antistaphyloccocenserum. Deutsche Medicinische Wochenschrift, XXIX, 195.

*Quetelet, A. I846. Lettres . . . sur la théorie des probabilités, appliquée aux sciences morales et politiques. Bruxelles. I846.

Richardson, O. Igor. Pseudo-Pneumococci in lobar pneumonia. Journal Boston Society of the Medical Sciences, V, 499. 
Rreke, H. I904. Beiträge zur Frage der Arteinheit der Streptokokken. Centralblatt für Bakteriologie, Abteilung, I, Originale XXXVI, 321 .

Ripley, W. Z. I899. The Races of Europe. New York. I899.

Robinson, B. L. Igo6. The Generic Concept in the Classification of the Flowering Plants. Science, N. S. XXIII, 8I.

Rodet, A., and Courmont, J. I89o. Sur les microbes de l'ostéomyélite aiguë juxta-épiphysaire. Comptes rendus hebdomadaires des séances et mémoires de la société de biologie. Serie IX. Tome II, I86.

Roscoe, H. E., and Lunt, J. I892. Contributions to the Chemical Bacteriology of Sewage. Philosophical Transactions of the Royal Society of London. B. CLXXXII, 648.

*Rosenbach. I884. Mikroorganismen bei den Wundinfektionskrankheiten des Menschen. Wiesbaden. I884.

Rosenow, E. C. Igo4. Studies in Pneumonia and Pneumococcus Infections. Journal of Infectious Diseases, I, 280.

Ruediger, G. F. I906; a. The cause of green coloration of bacterial colonies in blood-agar plates. Journal of Infectious Diseases, III, $66_{3}$.

Ruediger, G. F. I906; b. The Streptococci from scarlatinal and normal throats and from other sources. Journal of Infectious Diseases, III, 755 .

RŮžIČKA, S. I899. Vergleichende Studien über die. Bacillus pyocyaneus und den Bacillus fluorescens liquefaciens. Archiv fiir Hygiene, XXXIV, I40.

Sartirana, S., and Paccarano, A. I905. Der Streptococcus bombycis in Bezug auf die Aetiologie der Auszehrung und Schlaffsucht der Seidenraupe. Centralblatt für Bakteriologie, Abteilung I, Originale, XL, 207.

ScHANZ, F. I904. Ueber die Variabilität der Gonokokken. Deutsche medicinische Wochenschrifi, XXX, 350. 
Schierbeck, N. P. Igoo. Ueber die Variabilität der Milchsäurebakterien mit Bezug auf die Gärungsfähigkeit. Archiv fiir Hygiene, XXXVIII, 294.

Schläfrig, A. IgoI. Ueber eine pathogene Sarcine. Wiener klinische Wochenschrift, XIV, I025.

SchneIder, P. I897. Die Bedeutung der Bakterienfarbstoffe für die Unterscheidung der Arten. Arbeiten aus dem bacteriologischen Institut der technischen Hochschule. zu Karlsruhe, I, 203 .

SсноттмüLler, I903. Münchener medizinische Wochenschrift, L, 849.

*Schröter. I886. Kryptogamenflora von Schlesien, III.

Schumacher, G. I906. Ueber den streptococcus mucocus und seine Unterscheidung von anderen Streptokokkenarten. Centralblatt für Bakteriologie, Abteilung I, Originale, XLI, 628.

Sieber-Schoumoff, N. O. I892. Recherches sur les streptococcus pathogènes. Archives des sciences biologiques. St. Petersburg. I, 265.

Siтtн, E. F. 1905. Bacteria in their Relation to Plant Diseases, Volume I. Carnegie Institute, Washington. I905.

Sмiтн, T. I900. Variation among Pathogenic Bacteria. Journal of the Boston Society of Medical Sciences, IV, 95.

Sommerfeld, P. 1903. Vergleichende. Untersuchungen über Antistreptokokkensera nebst einigen Bemerkungen über die Kultur und Virulenz der Streptokokken. Centralblatt für Bakteriologie, Abteilung I, Originale, XXXIII, 722.

Sorgente, P. Ig05. Weitere Untersuchungen über den Meningococcus. Centralblatt für Bakteriologie, Abteilung I, Originale, XXXIX, I.

Staehel, I904. Zur Biologie des Streptococcus mastitidis contagiosæ. Archiv für wissenschaftliche und praktische Tierheilkunde, $\mathrm{XXX}, 374$. 
Steiger, P. Ig04. Bakterienbefunde bei der Euterentzündung der Kuh und der Ziege. Centralblatt für Bakteriologie, Abteilung I, Originale, XXXV, 326.

Sullivan, M. X. I905. Synthetic Culture Media and the Biochemistry of Bacterial Pigments. Journal of Medical Research, XIV, Iog.

ThoRNDIKE, E. L. I904. An Introduction to the Theory of Mental and Social Measurements. New York. Igo4.

Trevisan. I889. I generi e le specie delle Batteriacee. Milano. I889.

Twort, F. W. I907. The Fermentation of Glucosides by Bacteria of the Typhoid-coli Group and the Acquisition of New Fermenting Powers by Bacillus dysenteriæ and other Micro-organisms. Proceedings of the Royal Society. Series B, LXXIX, 329.

UNNA, P. G. 1900. Versuch einer botanischen Klassifikation der beim Ekzem gefundenen Kokkenarten nebst Bemerkungen über ein natürliches System der Kokken überhaupt. Monatshefte für praktische Dermatologie, XXXI, I.

*VAN DE Velde. I894. Etude sur le mécanisme de virulence du staphylocoque pyogéne. La cellule, $\mathrm{X}$.

VAN Durme, P. т903. Ueber Staphylokokken und Staphylolysin. Hygienische Rundschau, XIII, 66.

*Van Treghem, P. E. L. I878. Sur la gomme de sucrerie. Annales des Sciences naturelles. Botanique, VII, I80.

VANnOD, T. I905. L'agar ordinaire, comme milieu de culture du gonocoque. Centralblatt für Bakteriologie, Abteilung I, Originale, XL, I62.

VEIEL, F. I904. Die Staphylokokken des chronischen Ekzems. Münchener mendizinische Wochenschrift, LI, I3.

Vernon, H. M. I903. Variation in Animals and Plants. London. 1893. 
Vincent, H. IgO2. Sur les variations morphologiques du streptocoque et sur un streptocoque ramifié. Archives de médecine expérimentale et d'anatomie pathologique, XIV, $5^{2 \mathrm{I}}$.

Walker, E. W. A., and Ryffel, J. H. I903. The Pathology of Acute Rheumatism and Allied Conditions. British Medical Journal, I903, II, 659.

Weaver, G. H. I904. Agglutination of Streptococci, especially those cultivated from cases of scarlatina, by human sera. Journal of Infectious Diseases, I, 9I.

Weichselbaum, A. I886. Ueber die Aetiologie der acuten Lungen- und Rippenfellentzündugen. Medizinische Jahrbücher. Wien. Neue Folge, I, 483 .

Weichselbaum. A. I887. Ueber die Aetiologie der akuten Meningitis cerebro-spinalis. Fortischritte der Medicin, V, 573.

Weichselbaum, A. Ig02. Ueber die literarischen Schicksale des "Diplococcus intracellularis meningitidis" und seine ätiologische Bedeutung. Centralblatt für Bakteriologie, Abteilung, I, Originale, XXXIII, 5 IO.

*Weigman. I889. Milchzeitung, No. 50, 982.

Weston, R. S., and Kendall, A. I. Ig02. Some Common Bacteria in American Streams, including some new Species isolated at New Orleans, Louisana. Transactions of the American Public Health Association, XXVII, Igor meeting, 402.

Whipple, G. C. I902. On the Physical Properties of Gelatin, with Reference to its Use in Culture Media. Technology Quarterly, XV, r27.

WidAL, F. Igo6. Streptococci et Érysipèle de la face. Nouveau Traité de médecine at de thérapeutique. Publié sous la direction de MM. P. Brouardel et A. Gilbert. Fasc. X. Paris. I906.

Winslow, C.-E. A., and Rogers, A. F. I905. A Revision of the Coccaceæ. Science, N. S. XXI, I905, 669. 
Winslow, C.-E. A., and Rogers, A. F. I906. A Statistical Study of Generic Characters in the Coccaceæ. Biological Studies by the Pupils of William Thompson Sedgwick, Boston, 1906; also Journal of Infectious Diseases, III, 485.

Wollestein, M. 1907. Biological Relationships of Diplococcus intracellularis and Gonococcus. Journal of Experimental Medicine, IX, $5^{88 .}$

Woods, F. A. 1906. Mental and Moral Heredity in Royalty. New York. 1906.

ZetrNow, E. 1907. Uber Froschlaichbildungen in Saccharose enthaltenden Flüssigkeiten. Zeitschrift für Hygiene, LVII, 154. Zopr. 1885. Die Spaltpilze. 3 Auflage. Breslau. 1885. 


\section{INDEX OF AUTHORS}

Albrecht, I 25.

Ali-Cohen, 39, 247.

Andrewes, 7, 24-27, 38, 74, 102, I60, I61, I64, 165, I66.

Aronson, 150.

Babes, 167 .

Baginsky, 148.

Baruchello, I43.

Baumann, I8I.

Beaton, I49.

Beattie, 149.

Behring, I46.

Berry, I II.

Bertarelli, 127.

Besser, I 29.

Bettencourt, I23.

Beyerinck, 8, 238.

Bigelow, Io.

Billroth, 133 .

Binaghi, 129.

Birge, I69.

Bockenheimer, 180.

Boxer, 152.

Brant, 49.

Bruce, 166.

Buerger, 52, II5, I20, I30.

Bumm, I22, 218, 244.

Bürgi, I94.

Burri, I32.

Catterina, 206.

Celler, I 28.

Chester, 3 4, I8, 33, 34, 35, 78, I0I, I10, 169, I76, 192, I99, 209, 210, 211, 219, 239.

Cienkowski, I34.

Clark, 7r.

Class, 127.

Claussen, 230.
Cohn, 35,38, I 33, I4I, I43, I 85 , 201, 209, 219, 223, 245.

Cole, I 49.

Collins, I 3 I.

Conn, 9, 68, 70, 175 .

Courmont, 173 .

Dammann, 132.

De Waele, 150.

Dudgeon, I02, 103.

Dunham, 6, 56, 128.

Duval, II5.

Dyar, $7,34,60,188,200,218,234$, $243,244,245,246$.

Ellis, 5I, 52 .

Engler, 35 .

Escherich, 169.

Eyre, II4, II8.

Fehleisen, 142.

Fischer, 32, 15 I.

Flügge, 3, 33, 35, 1 $21,202,215$,

Fox, II4. $232,236,244,245$.

Fraenkel, I8r.

França, I23.

Frankland, 35, 220.

Friedlander, Irg.

Frosch, 127.

Fuller, 4.

Gaffky, 202.

Gage, 4, 71, I87.

Galton, IO.

Ghon, $125,127$.

Goodsir, 226, 236.

Gordon, 74, I02, 156, I57, I66, 169, 182, 195, 198, 200, 201 , 22I, 222, 229. 
Gotschlich, 7, 9.

Gruber, 9, 230, 232, 235, 237, 238.

Guillebeau, I43.

Hall, 5 .

Hallier, 209.

Harrevelt, 169.

Hashimoto, I67.

Hastings, r69.

Heinemann, 144.

Henrici, I44, I65, 230, 232, 235.

Heubner, I 26.

Heyrovsky, II 9 .

Hill, 52.

Hinterberger, 248.

Hiss, III, II 2, I I6.

Hlava, 130.

Hölling, I43.

Horder, 7, 24-27, 74, 102, 160, I6I, I64, 165, 166.

Horrocks, 16.

Houston, 74, I58, 159, 170.

Howard, I30.

Jaeger, I 24, I25, I 26, I 27.

Johne, I32.

Johnson, 4 .

Jordan, D. S., I6.

Jordan, E. O., 4.

Kayser, I8r.

Kellogg, 16.

Kendall, 4.

Kern, 232.

Kerner, I5I, 153 .

Kindborg, I 7 .

Klopstock, I80.

Knorr, I46.

Kolle, I27, I74, I8o.

Konradi, I74.

Konrich, I8I.

Kruse, 7, 9, 70, I 16, I43.

Kurth, I29, 146, I66, I7I.

Kutscher, I8r.

Leathem, II4, II8.

Le Gros, 35, I45, 153.

Lehmann, 35, 203, 230, 247.

Leichmann, 60, I67.
Lepierre, I 25, I 26.

Lewis, II 5.

Libman, III, 125.

Liesenberg, I36, I37.

Lindner, 230, 232.

Lingelsheim, 145, I $_{53},{ }_{54}$.

Lipstein, 48, I 73, I75, I79, I8I, I82.

Loeb, I8I.

Loewenberg, 203.

Longcope, I I 2, I I4, I3O.

Lubinski, I74.

Lunt, 146.

MacCallum, I69.

Macchiati, I44.

Manegold, 132 .

Marmorek, I47.

Massini 8.

Maurea, 248.

Menge, 224.

Meyer, A., $5 \mathrm{I}$.

Meyer, F., I50.

Migula, I, 4, I8, 28, 32, 33, 34, 35, $36,39,70,78$, IоI, I Iо, I34, I45, I70, 176, 192,-199, 203, 209, 2IO, 2II, 2I5, 220, 226, 230, 235, 239, 243, 247, 248.

Moberg, I76.

Molisch, 23, 108.

Moser, I48.

Muir, I4I.

Nägeli, I.

Nedrigailow, $\mathrm{I}_{5} \mathrm{r}$.

Neisser, A., I $2 \mathrm{I}$.

Neisser, M., 8, 48, I73, I75, I79, I80, I8I, I82.

Nencki, 62.

Neufeld, I48.

Neumann, 8, 35, 68, I30, I75, 203, 230, 247 .

Norris, II4.

Oestern, I73.

Ogston, 172.

Ortmann, A. E., I6.

Ortmann, P., I 29.

Otto, I74, I80, I90. 
Paccarano, I43.

Page, 156 .

Paine, I48.

Pansini, II6.

Pappenheimer, II4.

Park, II3, I3I.

Pasquale, I46, I7I.

Passet, I72.

Pay, I5o.

Pearson, io.

Peckham, I6.

Perkins, I30, I5o.

Pfeiffer, I 27.

Phelps, 4.

Pirquet, I48.

Pitfield, 207.

Poynton, I48, I49.

Prantl, 35 .

Prescott, I44.

Pröscher, I8I.

Quetelet, Io.

Rabenhorst, 35 .

Richardson, I30.

Rieke, I5 2.

Ripley, I2.

Ritchie, I4I.

Robinson, 13 .

Rodet, I73.

Rogers, I8, 36, 4I, 76, 90, 96, IIо, I $35,175,183,184$.

Roscoe, 146.

Rosenbach, I42, I72, I92, I98.

Rosenow, II 2.

Rovsing, 165 .

Růžička, 9.

Ruediger, $\mathrm{I}_{53}, \mathrm{I}_{5} 6$.

Ryffel, I49.

Sartirana, I43.

Schanz, I23.

Schierbeck, I7.

Schläfrig, 204 .

Schneider, 70.

Schottelius, 9.

Schottmüller, II2, I3I, I5I.
Schröter, 235.

Schumacher, I3r.

Sederl, I 27 .

Sieber-Schoumoff, 167 .

Smith, E. F., 72.

Smith, T., 7I.

Sommerfeld, I48.

Sorgente, I 26.

Staeheli, I43.

Steiger, 142.

Sternberg, 35 .

Stubenrath, 232.

Sugg, 150.

Sullivan, 9, 68, I75.

Thorndike, I2.

Trevisan, I23.

Twort, 17 .

Unna, 176,177 .

Van de Velde, I8o.

Van Durme, I80.

Van Tieghem, I34.

Vannod, I22.

Veiel, I8I.

Vernon, Io.

Vincent, 139, 145 .

Walker, I49.

Washbourn, II4, II8.

Weaver, 148 .

Wechsberg, I80.

Weichselbaum, I19, I23, I25, I26.

Weigmann, 132 .

Welch, 53, 200.

Weston, 4 .

Whipple, 57, $7 \mathrm{r}$.

Widal, $I_{55}$.

Williams, $I_{3}, I_{3}$.

Winslow, 18, 36, 41, 76, 90, 96, IIO, I35, I75, I83, I84.

Wollstein, I24, I27.

Woodhead, 35 .

Woods, I2.

Zettnow, 136 .

Zopf, 3I, I36. 



\section{INDEX OF SUBJECTS}

Acid production, 6I, 73, 74, 94, III, I35, I56, I68, I95I97, 221, 223, 233, 235.

methods of recording, 62 . methods of study, 61, 62 .

Acid production and chromogenesis, 83 .

Acidity, optimum, 63,64 .

Agar plates, colonies on, 57 .

Agar tubes, 57, 58, 59.

methods of study, 58,59 .

Agglutination, see Serum reactions. Air, cocci in, 157,158 .

Albococcus, Winslow and Rogers, $21,23,96,134,178,192-$ 208, 210, 2I 2, 223, 224, 226, 244 .

characters of, IOI-IO3, I93-I98. definition of, 104, 194.

virulence and immunity reactions, I79-182.

Albococcus candidus (Cohn), Winslow, 201, 202, 205, 206, 207.

epidermidis (Gordon), Winslow, 200, $201,218$.

pyogenes (Rosenbach), Winslow, I43, I67, I73, I94, I98, 200, 206.

synonyms of, I99.

tetragenus (Gaffky), Winslow, 202-205, 207.

Anaerobic sarcina, 238.

Anthropology, 12.

Ascococcus (Cohn), Winslow and Rogers, 23, 32; 37, 38, 39, 4I, 92, I33-I36, I37, I54, 237.

characters of, $133-136$.

definition of, 92,136 .
Ascococcus Billrothii, Cohn, I33. mesenteroides, Cienkowski, I34I $38,203$.

Aurococcus, Winslow and Rogers, 2I, 96, IOI, I72-I9I, I93, 210, $212,219,226,233,236$, 244.

characters of, 98-100.

definition of, I00, I79.

species of, I82-I9I.

virulence and immunity reactions, $179-182$.

Aurococcus aurantiacus (Schröter, Cohn), Winslow, I84-186, I89, 2 I9.

aureus (Rosenbach), Winslow, I83-184, I85, I86, I87, I89, $215,216,217$.

synonyms of, 184.

mollis (Dyar), Winslow, 186I88, I89, 21 7, 218, 233.

Bacilli, 225, 247.

Bacillus coli, Escherich, 2, 16, I7, 49.

diphtherice (Loeffler), Migula, 2, 50.

enteritidis, Gaertner, I7.

fluorescens, Flügge, 43, 50.

lactis acidi, Liebmann, I44.

megatherium, De Bary, 50.

mesentericus (Flügge), Migula,43. prodigiosus (Ehrenberg), Flügge, $6,8,134$.

proteus, Trevisan, $7 \mathrm{I}$. pyocyaneus, Gessard, 9. ramosus, Frankland, 56. rubellus, Okada, I7 I. salivarius septicus, Biondi, II9. Zopfii (Kurth), Migula, 43 . 
Bacteria, constancy of, 3 .

natural groups of, 3 .

systematic relations of, I, 24 . variability of, 2 . see Variations.

Bacterial characters, biochemical, 60.

cultural, 53,58 .

definiteness of, 28 .

frequency of, $13,24,29$.

latent, 23.

morphological, 27,45 .

physiological, 27.

Bacterial genera, characterization of, $\mathrm{I}_{4}, 30$.

Bacterial species, characterization of, IO, I 4, I $7,30,35,44$. grouping of, 33 .

Bacteridium, 32.

Bacteriology, systematic, 3 .

Bacterium Güntheri, 132, I44.

Beer, disease fermentations of, 230 , 232.

Biochemical reactions, see Bacterial characters, biochemical.

Biometry, see Statistical method.

Blood serum, 45, 54.

Bovine disease, see Cattle, diseases of.

Broth, growth in, 44, 54, 55, 196.

Capsule formation, 52 . effect of media and age on, 53 .

Capsulated albococci, 202-205.

Capsulated streptococci, I 29-132.

Carbohydrate media, 6I, 74, ro2IO3, III, II 4 , II 5 , I 30 , I 56 I59, I69, I82, I95-r97, 229.

Cattle, diseases of, $142,143,165$, I73.

Cell-division, see Fission.

Cells, form of, 45 .

grouping of, 45,73 .

effect of medium and age, 46 . method of recording, 46,47 .

size of, 47 .

effect of medium and age, 47 , 48.
Cells, size of, method of recording, 49.

Cheese, cocci in, I44, I6 5,230 , $232,233,235$.

Chromogenesis, 58, 67-70, 74, 78-8I, I70, I7I, I73-I76, 2I $9,22 I-223,225,226,232-$ $233,236-237,238$.

effect of environment on, 67,68 . method of studying, 69 .

variability of, 68 .

Chromogenesis and acid production, 83 .

gelatin liquefaction, 86 .

habitat, 8I.

nitrate reduction, 84 .

surface growth, 82 .

temperature relations, 85 .

Classification, decimal system, 4 . rational $5, I_{3}$.

Coagulation, see Milk, reactions in.

Coccaceæ, 3 I.

characterization of species, 35 .

genera of, 20, 32, 37, 39, 86-89, 94-98, 100, I78, I93, 21 2 , $227,228$.

grouping of species, 33-35.

subfamilies of, I9, 20, 36, 37, $76-79,86-89,178$, I92.

systematic relationships of, $\mathrm{x} 8$, $22,3 \mathrm{r}, 36,38,76$.

variability of, $\mathrm{I} 9$.

Cocci, isolation of cultures, 43 .

Coccus lactis viscosi, Gruber, $23 \%$.

Cohnia, 32.

Color chart, 69.

Committee on standard methods, $45,50,54,57,63,64,71$.

Coniferin, I58, 159 .

Correlation, 14. causes of, I 5 . significance of, $\mathrm{I} 6$.

Correlation between chromogenesis and other characters, $8 \mathrm{I}-86$.

Cultural characteristics, see Bacterial characters, cultural.

Curve of frequency, II, 29. 
Decolorization, see Milk, reactions in.

Dextrose broth, see Acid production.

Diplococcus (Weichselbaum), Winslow and Rogers, 23, 32, $37,39,4 \mathrm{I}, 49,53,90,9$ I, IIO-I38, 202, 226.

characters of, I IO-II9.

definition of, 9I, Iro.

relation to streptococcus, IIO-I I6. species of, II8.

Diplococcus catarrhalis, Frosch and Kolle, I 24, I25, I27, I 28 , I 29.

gonorrhace (Neisser), Flügge, I $21-I 23, I 24, I 28$.

intracellularis meningitidis,

Weichselbaum, I 23.

involutus (Kurth), Winslow, I I3, I 29-I32, I36, I38, I5I, 203. lanceolatus capsulatus, Kruse and Pansini, I I 7 .

lanceolatus capsulatus pneumonicus, Foà Bord, IIg.

luteus, Adametz, 224.

pneumonia, Weichselbaum, I го,

I I 2, I I 3 I I4, I I6, I I8, I I9I 2 I, I22, I24, I29, I3I, I 52, I63, I67.

roseus, Bumm, 244.

Weichselbaumii (Weichselbaum),

Trevisan, 40, I2I, I23-I 27 , I 28.

Dough, cocci in, 235 .

Earth, cocci in, 238.

Echidna, I4.

Fæces, streptococci in, I 58, I 59 .

See Intestine, bacteria in.

Fermentative powers, see Carbohydrate media.

Fission, 52.

Flagella, see Motility.

Foot-and-mouth disease, cocci in, I 29.

Fowls, diseases of, 132 .
Galactococcus, I43.

Gelatin, liquefaction of, $56,57,70$, 74, 100, I68-170, I84, I98, $215,217,218,231,243-244$, 246.

method of studying, $7 \mathrm{I}$. variability of, 70 .

Gelatin colonies, character of, 44 , $55,56,206,216,221,230$, 247.

Gelatin liquefaction and chromogenesis, 86.

Genera, see Bacterial genera. see Coccaceæ.

Glycerin agar, $5^{8}$.

Gonococcus, 32, 35. see $\mathrm{D}$. gonorrhœæ.

Gordon's tests, 158.

Grains, streptococci in, $\mathrm{I} 44$.

Gram stain, 50, 73, I68.

method of application, 50 . method of recording, $5 \circ$. variable results, 49 .

Gram stain and chromogenesis, 82.

Grouping of cells, see Cells, grouping of.

Growth, surface, and chromogenesis, 82 .

Growth, vigor of, $54,56,58,65,73$. method of recording, 59 .

Habitat, method of recording, 42, 72.

Habitat and chromogenesis, 8I.

Hanging-block, 52 .

Hemolytic power, II 2 , I5I-I53, I80-182, I89.

Horses, diseases of, I32, I65, 202.

Hyalococcus, 32.

Immunity, see Serum reactions.

Indol, production of, 63 .

Intestine, bacteria of, 232 .

Inulin, III, II4, II5, II6, I69. see Carbohydrate media.

Involution forms, 53 . 
Lactic acid, 60, 62, r67.

Lactic acid bacteria, see Milk, streptococci in.

Lactose, see Carbohydrate media.

Lactose agar, $5^{8}$.

Lactose broth, see Acid production.

Lampropedia, 32.

Leucocidins, r8o.

Leucocystis, 32.

Leucocystis cellaris, Schröter, I38.

Leuconostoc, 32, I34, I36, I37, I54. see Ascococcus.

Leuconostoc hominis, Hlava, Izo.

Lagerheimii (Ludwig), Migula, 138 .

Libman phenomenon, III, I3O.

Liquefaction, see Blood serum. see Gelatin, liquefaction of.

Macaroni, streptococci in, I44.

Malta fever, 167 .

Mannite, 158, I59, 160, 169.

Meal, cocci in, 235.

Meningococcus, see $D$. Weichselbaumii.

Merismopedia, 32.

Merismopedia flava varians, Dyar, $2 \mathrm{r} 8$.

fragilis, Dyar, 246.

mollis, Dyar, 188.

rosea (Bumm), Dyar, 244.

Merista, 32.

Metacoccaceæ, $37,38,90$, 104. definition of, 90 .

Methods, standard, see Committee on Standard Methods.

Micrococcus (Hallier), Winslow and Rogers, 22, 32, 33, 34, $37,39,41,96,98$, 104-106, 109, I34, I76, 191, 192, 193, $205,207,209-225,226,236$, 239, 24 I, 244.

characters of, 104-106, 212, 213, 214 .

definition of, 106, 212.

subdivisions of, 209-2II, 2 I4.

Micrococcus achrous, Migula, 224. acidi lactici, Marpmann, I65.
Micrococcus acidificans, Migula, I99.

agilis, Ali-Cohen, 51, 247, 248.

albatus, Kern, I99.

albescens, Henrici, r99.

albicans tardissimus, Flügge, I 29.

albocereus, Migula, 205.

albus, Sternberg, 199.

alvi, Chester, 199.

amarificans, Migula, I99, 204.

annulatus, Kern, 2 I6.

aquatilis, Bolton, 221, 223, 224.

aquatilis, Vaughan, 224.

aqueus, Migula, 184.

argenteus, Migula, 184.

ascoformans, Johne, 132, 202.

asper, Migula, 205.

aurantiacus, Cohn, 79, 185 .

aureus, Migula, I84.

badius, Lehmann and Neumann, 216.

Beigelii (Rabenhorst), Migula, 208.

Beri-Beri, Pekelharing, I8+.

bicolor, Kern, 246.

Biskra, Heydenreich, 184 .

bombycis, Pasteur, I43.

botryogenus, Rabe, 202.

brevis (Babes), Migula, I65.

butyri (v. Klecki), Migula, 220.

butyricus (v. Klecki), Migula, 220.

candicans, Flügge, 221, 223-224, 237.

synonyms of, 224 .

candidus, Cohn, 201, 223.

canescens, Migula, 224.

canus, Migula, 205.

carneus, Zimmermann, 246, 247.

carnicolor, Frankland, 246.

catarrhalis, Frosch and Kolle,

35.

cerasinus (Heim), Migula, 246.

cereus (Passet), Migula, 220.

cerinus, Henrici, 2 I6.

chlorinus, Cohn, 225.

chryseus, Frankland, I84.

cinnabareus, Flügge, 246. 
Micrococcus cinnabarinus, Zimmermann, 247.

cirrhiformis, Migula, 206.

citreus (Dyar), Winslow, 2I 7218, 221, 233, 234.

citreus, Sternberg, 220.

citreus agilis, Menge, 224.

citreus conglomeratus, Flügge, 218.

citreus liquefaciens, Unna, 2 I6.

citrinus, Migula, 2I6.

coccineus, Migula, 246.

commensalis (Turró), Migula, 220.

concentricus, Zimmermann, 224. confluens, Kern, 216.

conglomeratus (Flügge), Migula, 216, 218.

conjunctivitidis (Gombert), Migula, 2 I6.

corallinus, Catani, 246.

coralloides, Zimmermann, I99.

coronatus, Migula, 216.

corrugatus (Dyar), Migula, 216. coryze (Hajek), Migula, 205.

cremoides, Zimmermann, 2 I6.

cremoides aureus, Dyar, I84.

cretaceus, Henrici, 224.

cumulatus, Kern, 243, 246.

cuniculorum, Schröter, 208.

cupularis, Migula, 216.

cupuliformis, Migula, 220.

cyclops, Henrici, 224.

Dantecii, Chester, 246.

decalvans (Thin), Schröter, 208.

decolor, Migula, I 7 O.

dendroporthos, Ludwig, 208.

desidens (Flügge), Migula, 216.

diptericus, Cohn, I4I.

dissimilis, Dyar, 200.

eburneus, Henrici, 224.

excavatus, Kern, 220.

exiguus, Kern, I99.

expositionis, Chester, 216.

faviformis (Flügge), Migula, I99.

fervidosus (Adametz), Migula, 205.

flavens, Henrici, 216.

flavescens, Henrici, 216.
Micrococcus flavidus, Henrici, 2 I6. flavovirens, Migula, 220.

flavus (Flügge), Migula, 215217,2 I $, 221,23 I$.

synonyms of, $2 \mathrm{I} 6$.

flavus liquefaciens, Unna, 2 I6.

fotidus, Klamann, I99.

fragilis (Dyar), Migula, 243, 246.

Freudenreichii, Guillebeau, I99, 204.

fuscus, Eisenberg, 225.

galbanatus, Zimmermann, 216.

gelatinogenus, Bräutigam, 204, 205.

gigas, Frankland, 2 I6.

gilvus, Losski, 220.

globosus, Kern, 224.

grossus, Henrici, 224.

gummosus, Happ, 204, 205.

gummosus, Kern, 220.

hamatodes, Babes, 247.

hæmorrhagicus (Klein), Migula, I99.

Hauseri (Rosenthal), Migula, 224.

helvolus, Henrici, 220.

humidus, Migula, 224.

hyalinus, Ehrenberg, 208.

imperatoris, Roze, 208.

inconspicuus, Henrici, 224.

influenze, Migula, I99.

Iris, Henrici, 224.

Jongii, Chester, 220.

Kefersteinii, Chester, 246.

lactericeus, Freund, 246, 247.

lacteus, Henrici, 199.

lactis, Chester, 224.

lactis, Sternberg, I99.

Lembkei, Migula, 220.

lentus, Migula, I99.

licheniformis, Kern, 220.

liquefaciens (Flügge), Migula, I99.

liquidus, Migula, I7o.

lobatus, Migula, I84.

luridus, Kern, 220.

luteolus, Henrici, 216.

luteus (Cohn), Migula, 79, 2I922I, 224. 
Micrococcus luteus,

synonyms, 220.

lutosus, Kern, 216.

madidus, Migula, I84.

magnus, Rosenthal, I29.

mastitis, Chester, I99.

melitensis Bruce, 167 .

mucilaginosus, Migula, I38, I99.

nacreaceus, Migula, 224.

nasalis, Hack, 224.

nitidus, Kern, I99.

nivalis, Chester, 224.

niveus, Henrici, 224.

nubilus, Migula, 205.

nuclei, Roze, 208.

obscanus, Kern, I98.

ochraceus, Rosenthal, 220.

ochroleucus, Prove, 224.

odoratus, Henrici, 224.

odorus, Henrici, 224.

olens, Henrici, 2 I6.

orbicularis, Ravenel, 2 I6.

orbiculatus, Wright, 220.

ovalis, Escherich, 205.

ovis, Migula, I99.

pallens, Henrici, 205.

pannosus, Kern, 224.

parvus, Migula, 224.

persicus, Kern, 243, 246.

phosphoreus, Cohn, 225.

plumosus, Eisenberg, 220, $22 \mathrm{I}$.

polymyositis, Martinotti, I99.

polypus, Migula, 206.

prodigiosus, Cohn, 225, 247.

progrediens, Schröter, 208.

pseudocerevisice, Migula, I65.

pseudosarcina, Migula, I99.

pultiformis, Kern, I99.

punctatus, Migula, I99.

pyogenes, Lehmann and Neumann, I83.

quaternus, Migula, I99.

radiatus, Flügge, I99, 206.

radiosus, Kern, I99.

Reesii, Rosenthal, I99.

resinaceus, Kern, 220.

rhenanus, Migula, I99.

Rheni (Burri), Chester, I99.
Micrococcus rheumaticus, Beaton and Walker, I49, I66.

rhodochrous, Zopf, 246.

rosaceus, Frankland, 246.

roscidus, Migula, 224.

roseo-persicinus, Migula, 246.

rosettaceus, Zimmermann, 224.

roseus, Eisenberg, 243.

roseus, Flügge, 243, 244, 246.

rubellus, Migula, 246.

rubens, Migula, 243, 246.

rubescens, Migula, 246.

rubiginosus, Kern, 246.

rugatus (Weichselbaum), Migula, 205.

rugosus, Chester, 216.

saccatus, Migula, r99.

salivarius (Biondi), Migula, 205.

sarcinoides, Migula, 206.

scariosus, Migula, I99.

septicus, Cohn, I4I.

serratus, Migula, 205.

siccus, Migula, I85.

similis, Dyar, 205.

simplex, Wright, I99.

sordidus, Schröter, 224.

Sornthalii, Adametz, I32.

stellatus, Frankland, 206, 220, 22 I.

strobiliformis, Migula, 220.

subcanus, Migula, 224.

subcarneus (Kern), Migula, 246.

subcitreus, Migula, 2 I6.

subcretaceus, Migula, I99.

subflavidus, Migula, I84.

subflavus (Bumm), Migula, 2 I6. subgilvus (Henrici), Migula, 220. subgramulatus (Freund), Migula, 2 I6.

subgriseus, Migula, I99. sublacteus, Migula, I99. sublilacinus, Migula, 246. subniveus (Henrici), Migula, I99.

subochraceus, Migula, 2 r6.

subroseus, Migula, 246.

subtilis, Migula, I29.

succulentus, Henrici, 224.

sulfureus, Zimmermann, 220. 
Micrococcus tardigradus, Flügge, 220.

tardissimus (Flügge), Migula, 205.

tardus (Unna, Tommasoli), Migula, 2I6, 224.

tenacitis, Chester $22 \mathrm{I}$.

tenuis (Rosenbach), Migula, 205.

tenuissimus (v. Besser), Migula, 205.

tetragenus, Gaffky, 202.

tetragenus-citreus, Vincenzi, 22 I. tetragenus mobilis ventriculi, Mendoza, 207.

tetras, Henrici, 224.

trachomatis, Migula, 205.

tuberculosus, Migula, 224.

typhoideus, Migula, r99.

urece, Cohn, 206.

urea liquefaciens, Flügge, 206.

Urugua, Chester,'216.

utriculosus, Migula, r99.

vaccinea, Cohn, $\mathrm{r} 4 \mathrm{r}$.

versatilis, Sternberg, 2r6.

versicolor, Flügge, 221.-

vesica, Heim, 224.

vesiculiferus, Migula, $22 \mathrm{r}$.

vini (Kramer), Migula, r99.

violaceus, Cohn, 225.

viridis-flavescens, Güttmann, $22 \mathrm{I}$.

viticulosus, Flügge, 204, 205.

xanthogenicus (Friere), Stern-

berg, r99.

zonatus, Henrici, 224.

Microhaloa, 32 .

Milk, reactions in, 6o, $\mathrm{r}_{5}^{8}, 159$.

slimy fermentation of, ${ }_{3} 2,{ }_{2} 38$. streptococci in, 143 , I59.

Monas, 32.

Morphological characters, see Bacterial characters, morphological.

Motility, 39, 5r, 206-208, 213, 224, $247,248$.

Mouth, cocci in, I28, I 29, I $56, \mathrm{I}_{5} 8$, 202.

Mutations, 8.
Nährstoff agar, 48, 53, 54 .

Neutral-red, r $_{58}, \mathrm{r}_{59}$.

New York Health Department Investigation, $\mathrm{x} 3-\mathrm{Ir} 6$.

Nitrate-reduction and chromogenesis, 84 .

Nitrates, reduction of, $63,74,186$, $187,200,217,218,233,234$, 240, 244-245, 246.

Nomenclature, Linnæan, $17, x 8$.

Nostoc, $\mathrm{r} 34$.

Ontogenetic species, $\mathrm{x} 6, \mathrm{I} 7$.

Optimum temperature, see Temperature relations.

Orange strains of Micrococcus, 219 .

Sarcina, 232-233.

Ornithorhynchus, 14.

Oxygen, relation to, $59,64,65$.

Packet-formation, ro4, 203-204, 226, 227, 24r-243, 246.

Paracoccaceæ, Winslow and Rogers, $37,38,90,223$.

definition of, 90 .

Paratyphoid bacillus, 8 .

Pathogenicity, r94-r95, 200.

systematic value of, 44 .

see Serum reactions.

Pediococcus, 32.

Pediococcus damnosus, Claussen, 230.

perniciosus, Claussen, 230.

Photobacterium indicum, Beyerinck, 8.

Pigment-formation, see Chromogenesis.

Pigments, chemical composition of, 70,247 .

Planococcus, 32, 33, 39, 209.

Planococcus casei, Migula, 207.

Planosarcina, 32, 33, 39, 248.

Pleomorphism, $\mathrm{x}$.

Pneumococcus, see $D$. pneumonice.

Potato, growth on, 45,54 .

Proteus group, 56.

Pyogenic cocci, see Staphylococci.

Races of men, I2.

Raffinose, see Carbohydrate media. 
Rheumatism, cocci associated with, I $48,150,166$.

Rhodococcus, Winslow and Rogers, $22,23,96,98,107-109,207$, 210, 21 2, 227, 238, 239-248. characters of, 107-109, 239-242. definition of, rog, 242.

Rhodococcus fulvus (Cohn), Winslow, 245-246, 248.

roseus (Flügge, Dyar), Winslow, 244-246, 248.

Saccharose, see Carbohydrate media.

Salicin, I58, I59, I6r, I69.

Salicylic acid, production of, 167 .

Saliva, see Mouth, cocci in.

Sarcina (Goodsir), Winslow and Rogers, 22, 23, 32, 33, 37, $39,4 \mathrm{r}, 55,64,96$, 104-106, IO9, 203, 204, 226-238, 239, 24I, 244.

characters of, 104-106, 226-229. definition of, 106, 228. subdivisions of, 230.

Sarcina acidificans, Migula, 235.

alba, Zimmermann, 236.

albida, Gruber, 236.

alutacea, Gruber, 236.

aurantiaca, Flügge, 232, 236.

aurea, Macé, 232.

aurescens (Henrici), Gruber, 232.

baccatus, Migula, 232.

bicolor, Kern, 232.

butyria, Migula, 233, 235.

candida, Lindner, 236.

canescens, Stubenrath, 232.

carnea, Gruber, 246.

casei (Cervina), Stubenrath, 238.

citrea, Winslow, 233, 234.

citrina, Gruber, 235.

devorans, Kern, 236.

equi, Stubenrath, 232.

erythromyxa (Overbeck), Chester, 246.

flava, De Bary, 23I-233, 234.

synonyms of, 232.

flavescens, Henrici, 232.
Sarcina fusca, Gruber, 238. gasoformans, Gruber, 238. gigantea, Kern, 232. incana, Gruber, 236. incarnata, Gruber, 246. intermedia, Gruber, 235. intestinalis, Zopf, 238. lactea, Gruber, 236. lactis, Chester, 232. Lembkei, Migula, 232. liquefaciens, Frankland, 23I, 232.

livida, Gruber, 235. livido-lutescens, Stubenrath, 232. lutea, Schröter, 235, 236. synonyms of, $235,236$. luteola, Gruber, 235 . marginata, Gruber, 235. maxima, Lindner, 238. meliflava, Gruber, 235 . minuta, De Bary, 236. mirabilis, Kern, 232. mobilis, Maurea, 248. nivea, Henrici, 236. olens, Henrici, 232. paludosa, Schröter, 238. persicina, Gruber 246. pulchra, Henrici, 236. pulmonum, Virchow, 236. radiata, Kern, 232. rosacea, (Lindner), Migula, 246.

rosea, Lindner, 243, 246. rubra, Menge, 246. striata, Gruber, 235. subflava, Ravenel, 232. sulfurea, Henrici, 235. superba, Henrici, 232. tetragena (Gaffky), Migula, 203, 236.

variabilis, Stubenrath, 232. variegata, Pansini, 236. velutina, Gruber, 235. ventriculi, Goodsir, 226, 236. vermicularis, Gruber, 236. vermiformis, Gruber, 235. Welckeri, Rossmann, 235. Sarcina-grouping, see Packet-formation. 
Scarlet-fever, cocci associated with, I $27, I_{4} 6, I_{4} 8, I_{5}$ O, I5 I, I66.

Schottmüller reaction, II 2, II6, I 5 I.

Sediment, see Broth, growth in.

Selection of fluctuating variations, 9.

Serum reactions, $72, \mathrm{I}_{47}, \mathrm{I} 48, \mathrm{I} 5 \mathrm{O}$ $152,180-182,189$.

Silkworm disease, cocci in, I43, I66.

Size, see Cells, size of.

Skin, cocci of, see Staphylococci.

Small-pox, cocci associated with, I 49 , I 5 I.

Species, see Bacterial species.

Spirillum rubrum, v. Esmarch, I7I.

Spore-formation, 52 .

Staining-reactions, 49. see Gram stain.

Standard method, see Committee on standard methods.

Staphylococci, 32, 79, 102, x 72 I77, I92, I95-198.

virulence and immunity reactions, I79-I82, I94-I95.

cereus albus, Passet, 205.

Staphylococcus epidermidis albus, Welch, I94 195, 200.

mastitidis, Guillebeau, I43. pyogenes albus, Rosenbach, 172 , I94, I95, I98.

pyogenes aureus, Rosenbach, I 72,183 .

pyogenes citreus, Passet, Igo, 197, 216.

Starch, action upon, 72.

Statistical method, IO-I3, I8, 24, I6o.

Statistical study of coccaceæ, $4 \mathrm{I}$. isolation of cultures, 43 . source of cultures, 42 .

Stomach, cocci in, 232, 236.

Streptococci, chromogenic, I 7o, I 7 r.

liquefying, I68-r70.

systematic relations of, 24-27, I60-I6I, I64.
Streptococcus (Billroth), Winslow and Rogers, 23, 24, 32, 33, $37,38,39,4 \mathrm{I}, 55,64,90$, 93-95, I 29, I34, I35, I39I6r, 237.

characters of, 93-94, I39-I4I:

definition of, 95, I4I.

relation to Diplococcus, IIо-Iェ6, I 18 .

species of, I45-I $50,{ }^{1} 53^{-1} 55$, I59-I60, I64-I7I.

Streptococcus agalactice contagiosa, Kitt, I42, I43.

anginosus, Andrewes and Horder, I63, I65.

aurantiacus (Bruyning), Chester, 185 .

bombycis (Béchamp), Cohn, I66.

Bonvicini, Chester, I68.

brevis, v. Lingelsheim, I45, I57, I64, 165 .

brevis liquefaciens, v. Lingelsheim, 154 .

brevis pharyngis, v. Lingelsheim, I 54.

Brightii, Trevisan, I68.

capsulatus Binaghi, I29, I3I.

carnis, Chester, I69.

coli, Chester, r69.

coli (Escherich), Migula, I70.

coli gracilis, Escherich, I54, I69.

conglomeratus, Kurth, I46, I 57 , I65, I66.

enteritis, Hirsh, 165 .

enteritis, Libmann, 165.

enteritidis, Escherich, 168.

equinus, Andrewes and Horder, I6r.

erysipelatos, Fehleisen, I42, x64, I65.

fecalis, Andrewes and Horder, I63, I65, I66, I70.

Fischeli, Chester, I69.

gracilis (Escherich), Lehmann

and Neumann, I69, I 70 .

gracilis (Escherich), Migula, I 70.

hollandicus, Weigmann, I32.

involutus, Kurth, I29. 
Streptococcus lacticus, Kruse, I43, I44, I65.

lanceolatus, Gamaleïa, IIg.

liquefaciens, Sternberg, I69.

mastitidis sporadica, Guillebeau,

I42.

medius, Gordon, ${ }_{5} 6$.

mirabilis, Roscoe and Lunt, I46, I65.

mitior seu viridans, Schottmüller, I I 2, I52, I64.

mitis, Andrewes and Horder, I62.

mucosus, Howard and Perkins, II3, II5, II6, I3O.

ochroleucus (Prove), Trevisan, 185.

Pastoriamus, Krassilschtschik, I66.

proteus, Chester, 205.

pyogenes, Andrewes and Horder, I62, I65, I66, I67.

pyogenes, Rosenbach, II 2, I I4,

II7, II9, I 20, I 24, I3I, I42,

I43, I44, I48, I 56, I64.

pyogenes seu erysipelatos, Schott-

müller, II 2, I 52 .

rugosus (Rovsing), Migula, I7o. salivarius, Andrewes and Horder, 62, I66.

sanguineus, Migula, I 70.

sanguinis-canis, Pitfield, 207.

scarlatina, Gordon, I66.

septicus, Chester, 169.

septicus (Babes), Migula, I7ò.

Sphagni, Migula, 166.

sputigenus, Migula, I32.

trifoliatus (Rovsing) Migula, I70.

urea (Rovsing), Migula, I 70. vini (Kramer), Migula, I 70.
Streptococcus of Étienne, 154. the saliva, I54.

Subfamilies, see Coccaceæ.

Sugar refineries, cocci in, 134,136 , 237.

Temperature relations, $65,66,74$. methods of study, 66 .

Temperature relations and chromogenesis, 85 .

Tetracoccus, 32.

Thermal death-point, see Temperature relations.

Turbidity, see Broth, growth in.

Urine-fermenting cocci, I65, 206.

Variability, see Bacteria. see Coccaceæ.

see $D$. pneumonice, varieties of. see $D$. Weichselbaumii, varieties of.

Variations, classes of, 6 .

continuous and discontinuous, 7 . correlation of, $\mathrm{I} 4$.

effect of environment upon, 7, I6, I73, I74, I75.

fluctuating, 9 .

frequency of, $\mathrm{r}_{3}$.

Virulence, see Pathogenicity.

Water, cocci in, $x 66, x 89$.

White strains of Micrococcus, 22I223.

Sarcina, 237.

Wine, slimy fermentations of, 138 .

Zooglea-forming cocci, 38, 92, I33$\mathrm{I}_{3} 8,2 \mathrm{O} 3-205,237$. 




\subsection{3}

20.

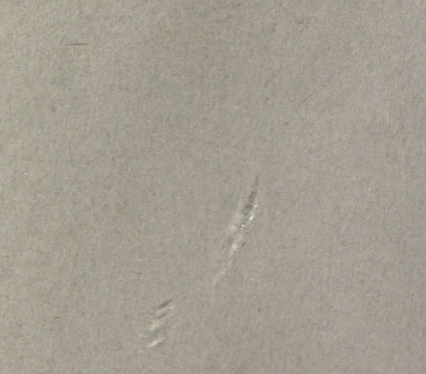

ing

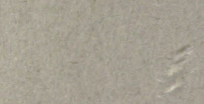

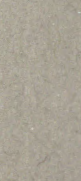

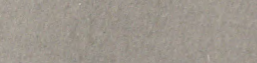

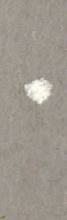

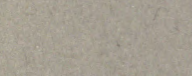

(5) 


\section{DAY USE RETURN TO DESK FROM WHICH BORROWED}

[D) (0):(1)

This book is due on the last date stamped below, or on the date to which renewed.

Renewed books are subject to immediate recall.

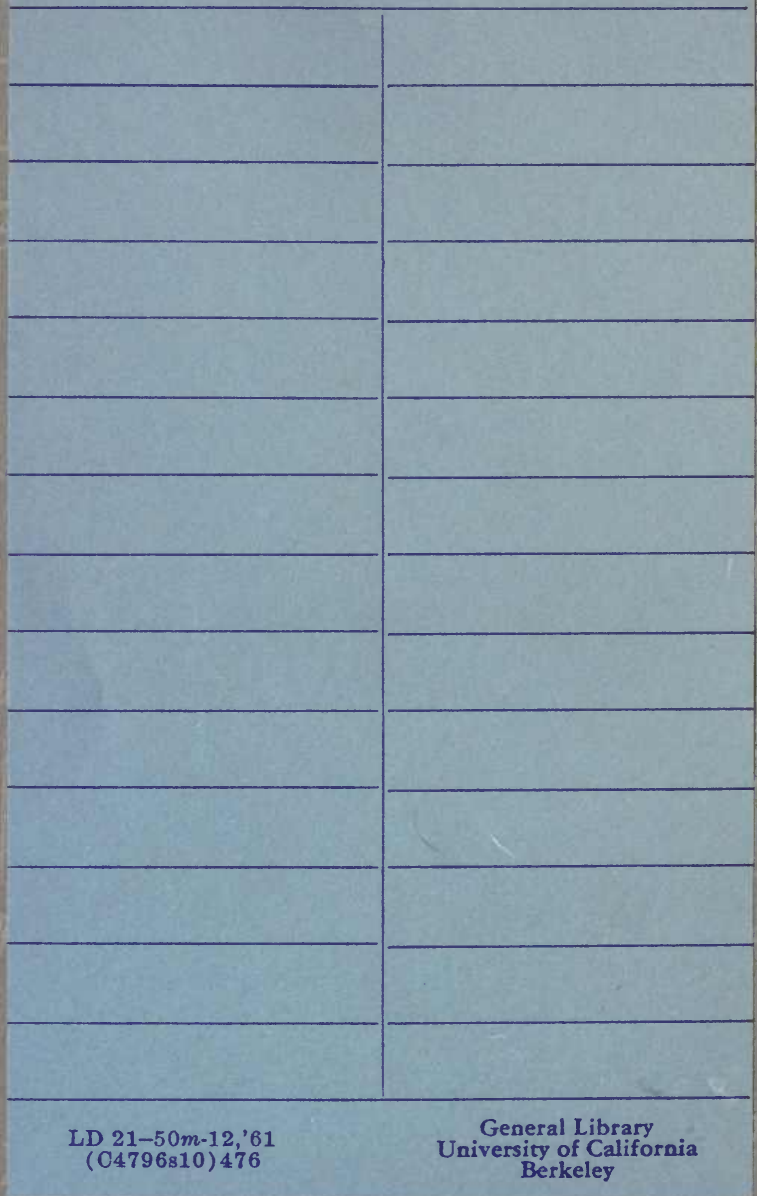




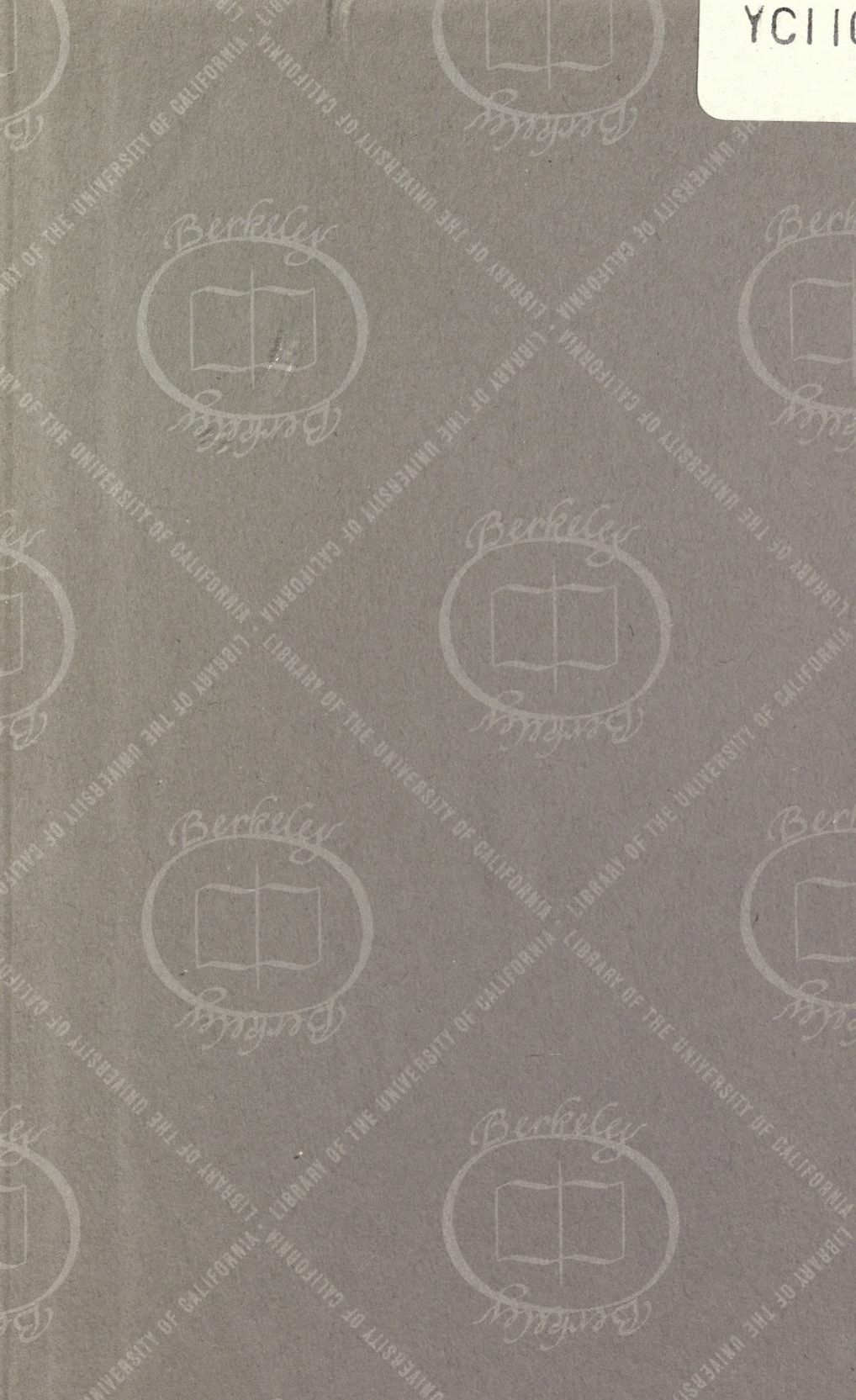


